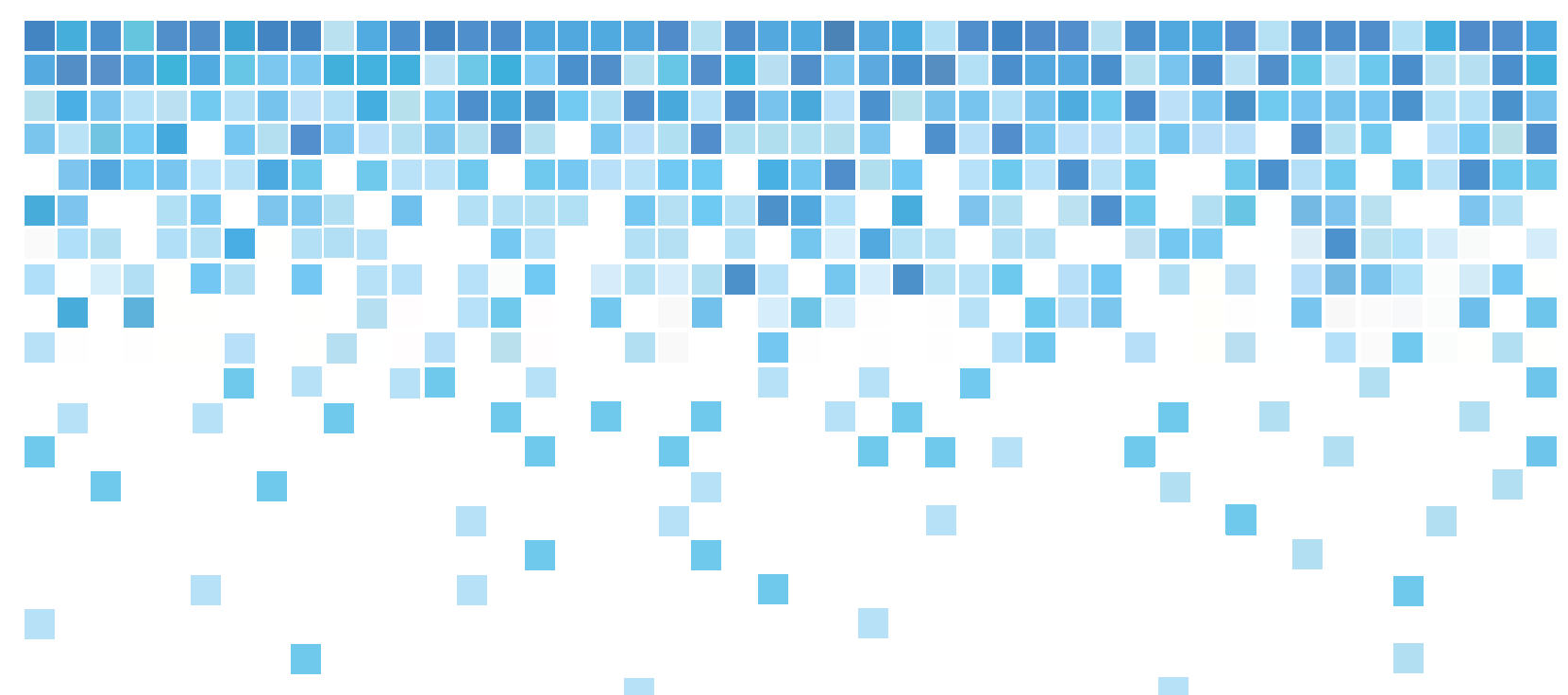

Energy-efficient Medium Access Control for Transmit Reference Modulation

Sarwar Morshed 

Energy-efficient Medium Access Control for Transmit Reference Modulation

Sarwar Morshed 


\section{Graduation committee:}

Chairman:

Prof. dr. ir. P.M.G. Apers University of Twente

Supervisors:

Prof. dr. ir. B.R.H.M. Haverkort University of Twente

Prof. dr. ir. G.J. Heijenk University of Twente

Members:

Prof. dr. J.L. van den Berg University of Twente

Prof. dr. ing. P.J.M. Havinga University of Twente

Prof. dr. M. Curado

University of Coimbra

Prof. dr. ir. S.M. Heemstra

Eindhoven University of Technology

Prof. dr. ir. M.J. Bentum

Eindhoven University of Technology

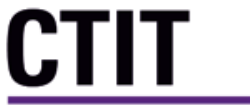

CTIT Ph.D.-thesis Series No. 17-445

Centre for Telematics and Information Technology

University of Twente

P.O. Box 217, 7500AE, Enschede

The Netherlands

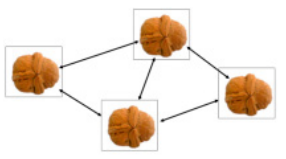

WALNUT - HARD TO CRACK

Wireless Ad-hoc Links using robust Noise-based Ultra-wideband Transmission

This research is supported by Dutch Technology

Foundation (STW) through Project number 11317

Cover design: A.K.M. Shahidur Rahman

Publisher: IPSKAMP Printing

Copyright (C) Sarwar Morshed

ISBN 978-90-365-4406

ISSN 1381-3617

https://doi.org/10.3990/1.9789036544061 


\title{
ENERGY-EFFICIENT MEDIUM ACCESS CONTROL FOR TRANSMIT REFERENCE MODULATION
}

\author{
DISSERTATION \\ to obtain \\ the degree of doctor at the University of Twente, \\ on the authority of the Rector Magnificus, \\ Prof. dr. T.T.M. Palstra, \\ on account of the decision of the graduation committee, \\ to be publicly defended \\ on Thursday, 02 November 2017, at 16:45 hrs \\ by \\ Sarwar Morshed \\ born on 12 October 1984 \\ in Dhaka, Bangladesh
}


This dissertation has been approved by the Supervisors:

Prof. dr. ir. B.R.H.M. Haverkort

Prof. dr. ir. G.J. Heijenk 


\section{Dedicated to my family}

To my parents, for their unconditional love

To my brother Muyeed, for his endless support

To my wife Rubaiya, for the last 11 wonderful years together

To my daughter Taiyeba, for being the source of my energy for the last 4 years 



\section{Acknowledgments}

I have heard, "Happiness is a journey, not a destination". Is it always true? I had a dream of being a PhD. Now that I have reached closer to the destination of my sine-wave journey towards achieving my dream, I would like to thank to those people without whom this could never happen.

First of all, I would like to thank my promotors Professor Dr. Ir. Boudewijn Haverkort and Professor Dr. Ir. Geert Heijenk providing me the opportunity to pursue my dream here in University of Twente. I am grateful towards both of you for believing in me. Thanks to Boudewijn for always welcoming me whenever I knocked on your door with any subject matter.

Geert, this thesis would not be possible without your constant guidance and endless support. I realized that the journey of being an independent researcher is similar to the training for being an independent driver with an experienced and certified trainer. Geert was that mentor for me from whom I received the most important training. I am grateful to you Geert.

I am thankful to my family, especially to my dearest and beloved parents Md. Abdul Hamid and Sharifa Akhter for being the constant source of strength for me. I am lucky to have both of you in my life. I am grateful for your relentless love, inspiration, sacrifice, and prayers. Muyeed, thanks for being the best brother. I turned to you in the toughest moments, and you were always there for me. I would like to thank Aditi for such a wonderful gift Mahrus, who is the source of joy for me from far away land.

Rubaiya, you are the light of my eyes, and the moon of my nights. The 11 years we have spent together seems to have passed like a blink of an eye. Thanks for walking together with me in the journey of life. This achievement of mine would never be possible without your companionship. 
Taiyeba, you made my life complete. Looking at you and spending time with you gave me enormous energy. One simple hug from you after returning from work removed all my tiredness. Just when I am writing this paragraph, coincidentally you just came to me and kissed me all over in my face and hands. Thanks Taiyeba for being the source of my energy for the last 4 years.

Thanks Siraz Kabir and Suraya Kabir for being the elder brother and sisterin-law. I do not have enough words to describe the supports that I received from Siraz Zubair. I am thankful to Hasib Mustafa and Saidul Chowdhury Tuhin. I feel honored to have younger brothers like you. Tania Apu, Joy bhai, Joey, Adi - I always enjoyed spending time with you. Thanks to my brotherin-law Zubair Mannan for your sudden visits from Germany. I have spent wonderful time in Enschede with Reza Khan, Ashif, Kallol, Mahua, Kushal, Labonno, Razu, Shaikat, Mahdin. Thanks to Ostok+ for being the extended family in The Netherlands - Ashiq bhai, Luna, Mahrus and Aroosh, Nayan, Iti, Nibras, Munzur, Maryam, Nusaibah. I feel myself lucky for such a wonderful Bangladeshi community across the whole country of The Netherlands.

I am thankful towards my colleagues in DACS. Thank you Mitra Baratchi for your guidance as a Post-doc. Bernd Meijerink, I really enjoyed your company in the room. Thanks for all the brain-storming sessions, and information about anything I required in my day to day life in The Netherlands. I will always remember the time that I enjoyed with Martijn, Wouter, Anja, Hamed, Mozhdeh, Morteza, Ricardo and Jair. Thanks Jenette Rebel-de Boer for all of your helps as you always tried to give your best. I am grateful to Dr. Pranab Mandal for his contribution. Thanks to Ibrahim, Dawei, Arjan, Ronan and Mark for your cooperation while working in the WALNUT project.

Finally, I would like to thank the Almighty. All credit goes to God Almighty.

Sarwar Morshed

Enschede, October 2017. 


\section{Abstract}

A Wireless Sensor Network (WSN) consists of sensor nodes distributed across an area to collect and communicate information. Research in the field of WSNs became interesting in recent times because of the challenges and constraints these WSNs impose, e.g., limited available energy, achieve low complexity and low cost. These research challenges influence the design of the protocols of all the layers of the communication stack, especially the Medium Access Control (MAC) layer protocol. The MAC protocol is responsible for controlling the transceiver operation, which is the most energy consuming part of the wireless sensor node. Due to this fact, the transceiver is assigned to sleeping mode most of the time in a wireless sensor node to minimize the overall energy consumption of the WSN. In this case, the WSN requires an energy-efficient MAC protocol, which can provide reliable communication even though the sensor nodes are sleeping for most of the time.

The applications of WSNs require robust communication with low power consumption in the MAC layer, as well as in the physical layer. Transmit reference (TR) modulation in the physical layer offers a simple low power receiver architecture, achieves fast synchronization with short signal acquisition time, and provides multiple frequency offsets that can be used for multiple simultaneous communications. However, this modulation technique has a performance penalty at the transmitter side because of transmitting the reference signal together with the modulation signal, and at the receiver side because of their cross products as well as the interfering signals of multiple frequency offsets using the same spectrum. Hence, TR modulation is suitable for WSN applications requiring low power operations, if a suitable MAC protocol can be designed together with this modulation technique to exploit its advantages and minimize 
its drawbacks.

The main contributions of this dissertation are motivated by the research question of how to design a medium access control protocol to exploit the features of the underlying transmit reference modulation to provide energy-efficiency, traffic-adaptive behavior, and robust medium access control in a shared wireless environment.

We introduce a new energy-efficient MAC protocol, called TR-MAC, which exploits the benefits of the TR modulation in the underlying physical layer, and minimizes its drawbacks. We design an analytical model to analyze the energyefficiency of the TR-MAC protocol, and provide optimization mechanisms using the analytical model to minimize the overall energy consumption of the protocol operation. Further, we design an energy-efficient traffic-adaptive mechanism for the TR-MAC protocol such that it can accommodate traffic-adaptive behavior to deal with higher traffic load generated by sudden event-driven scenarios where a sudden event rapidly increases the traffic load within the network, which needs to be disseminated fast.

We utilize the multi-channel features provided by the underlying TR modulation, and design a multiple access control model to provide a fundamental insight into the performance of a MAC protocol with TR modulation using multiple frequency offsets for multiple communications. Finally, we provide a detailed design of the TR-MAC protocol, and analyze the protocol performance from the multiple access perspective.

The analysis of this thesis improves the understanding of the frequency offset based transmit reference modulation, and proposes a medium access control protocol customized for this unique modulation technique, named as the TR-MAC protocol. The TR-MAC protocol exploits the fast synchronization capabilities of the underlying frequency offset based transmit reference modulation. Energyefficiency is achieved in this TR-MAC protocol by using transceiver duty cycling mechanism and preamble sampling technique. Furthermore, this TR-MAC protocol achieves traffic-adaptive behavior while maintaining energy-efficiency by using a duty cycle adaptation algorithm. The multi-channel multiple access model using frequency offsets and the performance analysis of the TR-MAC 
protocol in a multiple access scenario show that the TR-MAC protocol together with frequency offset based TR modulation increases the throughput and efficiency of the system while maintaining energy-efficiency. This performance enhancement is achieved by exploiting the inherent multiple access capability of the transmit reference modulation, which uses multiple frequency offsets for multiple simultaneous transmissions. Because of the inherent power and performance limitations of frequency offset based transmit reference modulation, the TR-MAC protocol is suitable for low data rate and low-duty-cycle sparse WSN scenarios, for example, monitoring and tracking applications. 



\section{Samenvetting}

Een draadloos sensornetwerk (WSN) bestaat uit sensor nodes die verspreid over een gebied data verzamelen en onderling uitwisselen. Onderzoek op het gebied van WSN's is uitdagend door de combinatie van eigenschappen en beperkingen van deze netwerken: beperkte beschikbaar energie, bereiken lage complexiteit en lage kosten. Deze beperkte middelen hebben invloed op alle lagen van het ontwerp van de communicatieprotocollen. De grootste invloed is die op de Medium Access Control (MAC) protocollen. Een MAC-protocol is verantwoordelijk voor de aansturing van de transceiver, die verantwoordelijk is voor het zenden en ontvangen van signalen. Dit onderdeel verbruikt het meeste energie in een sensor node. Om energie te besparen is de transceiver het grootste deel van de tijd uitgeschakeld. Om het gehele WSN zo energie-efficiënt mogelijk te maken is een MAC-protocol nodig dat betrouwbare communicatie mogelijk maakt en de sensor nodes toch het grootste deel van de tijd kan laten slapen.

De toepassingen van WSN's vereisen betrouwbare communicatie met laag energieverbruik in de MAC en de fysieke laag. Transmit Reference (TR) modulatie op de fysieke laag staat het gebruik van een simpele radio-ontvanger architectuur toe met laag energie verbruik, snelle synchronisatie en de mogelijkheid om meerderde communicatiestromen tegelijkertijd te onderhouden. De nadeel van deze modulatietechniek aan de zenderijde is het versturen van een referentiesignaal tegelijk met het gemoduleerde signaal, en aan de kant van de ontvanger vanwege hun kruisproducten en interfererende signalen van meerdere frequentie-offsets die hetzelfde spectrum gebruiken. TR-modulatie is geschikt voor WSN-applicaties die een laag energie verbruik hebben als er een geschikt MAC-protocol ontworpen kan worden dat de voordelen van deze mod- 
ulatie techniek kan gebruiken terwijl de nadelige gevolgen geminimaliseerd worden.

De onderzoeksvraag die in dit proefschrift beantwoord wordt is: Hoe kunnen we een MAC-protocol ontwerpen dat de sterke punten van TR-modulatie gebruikt om energie-efficiënte, adaptieve en robuuste toegang tot het draadloze medium te geven in een omgeving met veel nodes.

Wij introduceren een nieuw energie-efficiënt MAC-protocol genaamd TR-MAC dat de voordelen van TR-modulatie in de onderliggende fysieke laag gebruikt en de nadelen minimaliseert. We ontwerpen een analytisch model om de energie-efficiency van het TR-MAC protocol te evalueren en optimaliseren het protocol aan de hand van dit model. Ook ontwerpen we een adaptief mechanisme voor TR-MAC zodat het om kan gaan met en zich aanpassen aan verschillende hoeveelheden verkeer. Dit is nodig omdat er zich situaties voor kunnen doen waarin een netwerk ineens grote hoeveelheden verkeer snel moet kunnen verwerken.

TR-modulatie maakt het tegelijkertijd gebruiken van meerdere kanalen mogelijk door verschillende offsets tussen de frequenties van het gemoduleerde signaal en het referentiesignaal te gebruiken. Om inzicht te geven in de prestaties van MAC protocollen voor TR-modulatie met meerdere kanalen ontwikkelen wij een wiskundig model dat de essentie van zulke protocollen beschrijft. Verder presenteren we een gedetailleerd ontwerp van het TR-MAC protocol en analyseren we dit op prestaties in een omgeving met veel gelijktijdige communicatiestromen. De analyses beschreven in dit proefschrift verbeteren ons begrip van het gebruik van TR-modulatie. Het in dit proefschrift voorgestelde MAC-protocol, genaamd TR-MAC, is ontworpen om deze modulatie techniek optimaal te gebruiken. Het TR-MAC protocol gebruikt het snelle synchronisatie mechanisme van de onderliggende TR-modulatie techniek. TR-MAC bespaart energie door het gebruik van zogenaamde transceiver duty cycling en preamble sampling mechanismen. Adaptieve duty cycling wordt gebruikt om TR-MAC aan te passen aan de hoeveelheid verkeer terwijl het energieverbruik geminimaliseerd wordt. Het multi-channel multiple access model in combinatie met de prestatie-analyse van TR-MAC, toont aan dat het TR-MAC protocol samen met TR-modulatie zorgt 
voor een verbeterde doorstroming en efficiëntie van het gehele systeem met laag energiegebruik. Prestatieverbeteringen worden behaald door gebruik te maken van de inherente multiple access eigenschap van TR-modulatie, waarbij meerdere communicatiestromen tegelijkertijd worden gerealiseerd door verschillende offsets tussen de frequenties van het gemoduleerde signaal en het referentiesignaal. Door zijn energie- en prestatie-eigenschappen is het TR-MAC protocol geschikt voor WSN communicatie met lage bandbreedte en lage activiteit, zoals applicaties voor monitoring en tracking. 



\section{Contents}

1 Introduction $\quad 1$

1.1 Motivation ........................ 2

1.2 Research and design challenges ... . . . . . . . . . . . . 6

1.3 Research objectives .................. 9

1.4 Contributions and thesis organization . . . . . . . . . . 10

2 Background and Related Work $\quad 13$

2.1 Importance of MAC protocols in WSNs . . . . . . . . . . . . . . 13

2.2 Transmit reference modulation . . . . . . . . . . . . . 17

2.3 Existing MAC protocols for WSNs . . . . . . . . . . . . . 20

2.4 Classification of asynchronous preamble sampling protocols . . 27

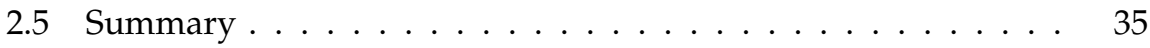

$3 \quad$ Energy-efficient medium access control 37

3.1 Motivation ........................ 38

3.2 TR-MAC protocol: design and operation . . . . . . . . . . 39

3.3 Analytically modelling TR-MAC protocol . . . . . . . . . . . 45

3.4 TR-MAC protocol optimization . . . . . . . . . . 53

3.5 Comparison with reference protocols . . . . . . . . . . . 57

3.6 Validation of analytical modelling . . . . . . . . . . . 62

3.7 Summary . . . . . . . . . . . . . . . 75

4 Traffic adaptation in MAC protocols 77

4.1 Motivation ........................ 78

4.2 Related work. . . . . . . . . . . . . . . . . 80 
4.3 Traffic adaptation in the TR-MAC protocol . . . . . . . . . 84

4.4 Results and analysis . . . . . . . . . . . . . . 94

4.5 Summary . . . . . . . . . . . . . . . . . . . 111

5 Modelling multi-channel multiple access using frequency offsets 113

5.1 Motivation . . . . . . . . . . . . . . . . . . . . 115

5.2 Related work . . . . . . . . . . . . . . . . . . . . . . . . 117

5.3 Modelling multiple access schemes . . . . . . . . . . . . . . . 118

5.4 Results and analysis . . . . . . . . . . . . . . . . . . . . 124

5.5 Summary . . . . . . . . . . . . . . . . . . . . . . . . 132

6 TR-MAC protocol: detailed design and performance analysis 133

6.1 TR-MAC protocol: detailed design . . . . . . . . . . . . . 135

6.2 TR-MAC protocol: multiple access perspective . . . . . . . . 148

6.3 Performance analysis . . . . . . . . . . . . . . . . . . . . . . . 164

6.4 Summary . . . . . . . . . . . . . . . . . . . . . . . 181

7 Conclusions and future work 183

7.1 Contributions and concluding remarks . . . . . . . . . . . . . 184

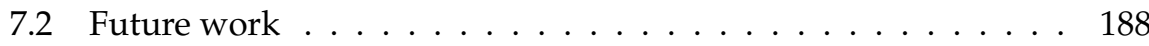

$\begin{array}{ll}\text { Bibliography } & 191\end{array}$

$\begin{array}{ll}\text { About the author } & 200\end{array}$

$\begin{array}{ll}\text { Acronyms } & 202\end{array}$ 


\section{CHAPTER 1}

\section{Introduction}

A Wireless Sensor Network (WSN) is a network of low power, low complexity, and energy-constrained sensor nodes distributed over an area to collect information, and communicate over a shared wireless communication medium [1]. The sensor nodes consist of sensors, computing devices, and communicating radio technologies to collaborate in the information dissemination activity towards other peers. Wireless sensor networks are widely seen as a key enabling technology to create an Internet of Things (IoT), which can support numerous applications, ranging from environmental, healthcare, home, military, and commercial industrial applications. As a result, WSNs have been researched, and gained popularity in the last two decades [2,3].

As of today, the main requirement of WSNs is low power consumption to allow a lifetime of a few years on a single small battery using energy harvesting [4]. Therefore, achieving energy-efficiency is crucial in WSNs. Communication technology is the dominating component in the energy consumption compared to the sensors and the computing devices within a sensor node. Low data rate applications are more suitable for WSNs, since energy consumption grows linearly with the number of bits exchanged. Among the other requirements that characterizes WSNs are: high application dependency, change of topology, variable traffic pattern, scalability, and high network dynamics $[1,5,6]$.

The wireless medium is inherently broadcast in nature. Hence, a wireless sensor network requires a highly optimized medium access control (MAC) protocol to establish successful communication links for data transfer. The prime role of 
a MAC protocol is to coordinate the access to the shared wireless medium for the sensor nodes of the wireless sensor network [2]. As a result, the medium access control is crucial to proper functioning of any communication system, hence is the focus of this thesis. The MAC protocol also determines the energy consumption of a wireless sensor node by specifying the listening, transmitting, and sleeping time [6]. Thus, MAC protocols play an important role in minimizing the overall energy consumption in a typical WSN. The aim of this thesis is to focus on the communication technologies of a WSN from the perspective of the MAC layer taking into account the transmit reference modulation technique in the physical layer, while targeting relevant applications.

The outline of this chapter is as follows. First, we present the motivation for this thesis. Afterwards, we describe the possible research challenges. Next, we formulate the research questions that we answer throughout this thesis. Following this, we present the contributions of this thesis. Finally, we elaborate on the thesis organization.

\subsection{Motivation}

WSNs have a wide range of applications that require robust communication with low power consumption [1,5]. Transmit Reference (TR) modulation, capable of achieving fast synchronization with short signal acquisition time, offers a simple low power receiver architecture, and can potentially meet the requirements of these applications $[7,8]$. As a result, the motivation for this thesis for designing a MAC protocol with TR modulation comes from the requirements imposed by the WSN applications, as well as, the capability of the underlying modulation technique to satisfy these requirements.

\subsubsection{Applications}

Wireless sensor networks consist of small and smart sensing and computing devices that are capable of sensing, e.g., movement, temperature, light, pressure, presence or absence of certain objects. As a result, a wide ranges of applications 
are possible with WSNs in our day-to-day life. Wireless sensor networks play an important role in the Internet of Things (IoT) applications in recent years.

- Environmental applications: Wireless sensor networks have several environmental applications that involve monitoring environmental conditions for habitat, tracking the movements of animals, monitoring the soil condition for irrigation and plants, monitoring green houses, monitoring toxic substances in drinking water, detecting flood, pollution, and forest fire, etc. [9]. In some of these applications, the WSN provides reliability and safety through monitoring; and in some of the applications, the WSN collects information for a longer period to observe significant internal variation.

- Healthcare applications: Applications of WSNs for healthcare include monitoring patients, especially critical patients with acute mental illness or disability [1]. Another WSN application can be monitoring old people to detect whether they have fallen. Furthermore, doctors and patients can carry small sensors that will enable them to locate other doctors or a critical patients' current location.

- Home applications: Embedding sensor nodes into the furniture and appliances of a typical home is now a reality with the advancement of the technology. These nodes can interact with each other or to the outside world via the Internet. The users can manage the devices efficiently through the use of the sensors both locally and remotely. Various domestic applications include sensor-driven lights, temperature control, monitoring security of the houses, monitoring water, gas, electricity, and wastage systems, etc. [9].

- Military applications: Low power, robust WSNs are very much suitable for military applications because of their capability to work in a harsh environment. WSNs can help the military to monitor their allies and enemies, collect information, perform surveillance of battlefield, detection of chemical, biological, nuclear weapons, etc. [1].

- Commercial applications: Many commercial applications rely on WSNs for 
their reliability and low cost. Some examples are monitoring inventory and warehouses, monitoring product quality, monitoring bridges, lifts, and critical structures, etc. The use of WSN in these industrial and commercial situations helps to detect failures at an early stage, thus enabling efficient preventive maintenance.

The ever-broadening horizon of WSNs has led to rapid growth in the market of smart building, intrusion detection, target tracking, and healthcare applications. A WSN has its own design requirements and resource constraints, and highly dependent on the application environment [3]. The design requirement of a WSN depends on the monitored environment, and influences the size of the network, topology, and deployment scheme. Ad-hoc deployment is generally preferred over planned deployment for a WSN. The topology of the network depends on the type of the application and environment. The resource constraints include low power requirement, short coverage area, and limited processing in a node. Therefore, the MAC protocols for WSNs need to meet the specific design and resource requirements offered by the applications and environments.

\subsubsection{Transmit Reference modulation in the underlying physical layer}

Dealing with the modulation of the data signal to the reference signal for the transmission in the medium is one of the key tasks of the physical layer, which is underneath the MAC layer in the Open Systems Interconnections (OSI) model. Transmit Reference modulation is a novel modulation technique in the physical layer that allows transmitting the reference signal together with the modulated signal [10]. TR modulation provides a simple low power receiver architecture with fast synchronization with inherent multiple access capability, and all together offers many opportunities to be exploited in the upper MAC layer [7]. The primary role of the MAC layer is to control the channel access mechanism of the shared wireless communication medium for multiple nodes. In this thesis, we investigate the use of TR modulation in the underlying physical layer from the MAC layer perspective to achieve energy-efficiency, robust connectivity, 


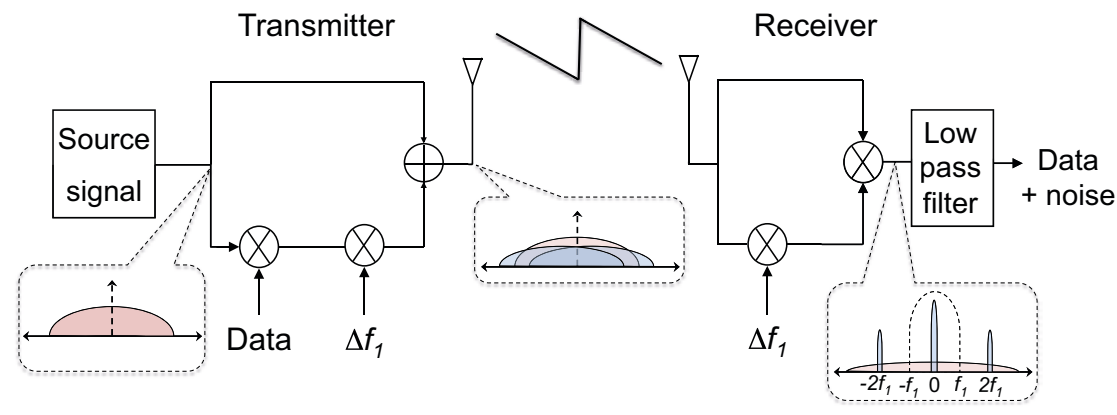

Figure 1.1: Transmit reference modulation

traffic adaptation, and medium access control for low data rate and low power operation for WSNs.

The transmitter using the TR modulation sends both the modulated and unmodulated signal with a known frequency offset [8], as presented on the transmitter side in Figure 1.1. In doing so, the transmitter consumes higher power than a traditional modulation technique, but this approach also enables the receiver with some possibilities to reduce the power consumption in the network. The receiver performs demodulation by correlating the received signal with a frequency shifted version of itself. Initially, the TR modulation was realized using time offset in the literature [10,11,12]. Afterwards, frequency offset based TR modulation was proposed $[7,13,14]$, which provided improved performance with simplified receiver architecture to implement on a chip.

There are several advantages of frequency offset based transmit reference modulation [15]. i) The receiver can restore the original signal quickly by correlating the received signal with a frequency-shifted version of the received signal. This is possible since all multi-path components contain identically distorted pulses, if the frequency offset is within the coherence bandwidth of the propagation channel. ii) The receiver can achieve faster synchronization with reduced signal acquisition time because of the correlation-based decoding, which is highly desirable for low duty cycle transmissions. iii) The receiver architecture becomes simple and energy-efficient without the need of a complex rake receiver 
technique, channel estimation, or power-hungry stable oscillators. iv) The TR modulation provides an inherent multiple access capability using multiple frequency offsets. v) The frequency offset based TR system is robust against frequency-selective fading.

While providing the above mentioned advantages, the TR modulation also comes with few drawbacks. The TR modulation consumes higher power on the transmitter side because of sending both the modulated and unmodulated signal in the channel [7]. On the other hand, the receiver using the TR modulation experiences an overall increase of the noise-floor because of the multi-user interference in the presence of a large number of active links with multiple frequency offsets. This noise is further increased at the receiver side by the self-mixing of the modulated and the unmodulated signals [87, 17, 15].

Considering all these interesting attributes of TR modulation in the physical layer and its radio technology implementation in circuit level, a customized MAC layer protocol is clearly required for energy-efficient robust reliable information dissemination by exploiting the advantages offered by the TR modulation while minimizing its drawbacks. This cross-disciplinary research work combining three research fields of the relevant low power robust MAC layer protocol design using the TR modulation in the underlying physical layer together with circuit level realization of this concept has been carried out in the WALNUT project funded by the Dutch Technology Foundation [18].

\subsection{Research and design challenges}

Wireless sensor networks have their own characteristics, which creates several challenges to design a MAC protocol. Wireless sensor networks can have diverse applications, as presented in Section 1.1.1, because of the large number of conceivable combinations of sensing, computing, and communication technologies. As a result, the MAC layer protocol is often customized to the specific application needs [1]. Furthermore, a customized MAC protocol is required to exploit the specific features of TR modulation in the underlying physical layer, as presented in Section 1.1.2. The MAC protocol needs to provide energy 
efficient connectivity with robust and traffic-adaptive communication including multiple access capability by using the characteristics of TR modulation. Designing an customized MAC protocol imposes several research challenges, which are discussed below:

- Energy-efficiency: Energy-efficiency is one of the most important factors that need to be considered in design of a WSN [9]. The lifetime of a sensor node depends on its battery; therefore, prolonging the battery lifetime has a deep impact on the lifetime of the network. That is why, the main challenge in sensor network research domain is to ensure robust communication with the goal to extend the network lifetime by minimizing energy consumption. One way to increase the battery lifetime of the wireless sensor node is to use energy harvesting, which refers to converting energy from the environment or other energy sources, e.g., motion, pressure, or heat, to electrical energy [4]. TR modulation consumes higher power than a general modulation technique to transmit individual bits, since the reference signal is also sent [7]. Furthermore, the receiver has a performance penalty because of the self-mixing of the modulated as well as the unmodulated signal and the interfering signals of multiple frequency offsets using the same spectrum [16, 17, 15]. As a result, achieving energy-efficiency in the upper MAC layer is crucial for TR modulation.

- Robust connectivity: The MAC protocol needs to provide reliable and robust connectivity despite of using any mechanism to increase energy-efficiency. Since communication dominates the energy consumption compared to sensing and computing within a sensor node, sometimes the amount of communication is minimized in WSNs. Furthermore, the MAC protocol makes the node to sleep a dominantly large amount of time to save energy. For example, transceiver duty cycling is used to increase energy-efficiency, where each node periodically switches between working and sleeping mode. Providing robust connectivity by coordinating the duty cycles of all sensor nodes within the network is one of the key challenges of a MAC protocol. 
- Traffic adaptation: Most often the MAC protocol achieves energy-efficiency by sacrificing some of the Quality of Service (QoS) parameters, for example, maximizing throughput or minimizing communication delays [6]. However, sometimes the specific application requires improvement of these QoS parameters. A monitoring application will have burst traffic caused by an event that needs to be quickly disseminated in the network. As a result, the MAC protocol requires adapting to traffic variations within the network while maintaining energy-efficiency.

- Multi-Channel multiple access control: The MAC protocol is responsible for the multiple access control for the shared wireless medium in WSNs. A system using frequency offset based TR modulation allows multiple nodes to transmit simultaneously and asynchronously without any mutual timing coordination by using different frequency offsets. Thus, a crucial challenge remains about the utilization of this inherent capability of TR modulation for multiple access control in the MAC layer to coordinate the shared use of the common wireless medium among the nodes of the WSN.

- High network dynamics: Wireless sensor networks have very high network dynamics due to the addition of new nodes, failure of nodes, battery drain-out, topology changes, and scalability, etc. [5]. For example, TR modulation has interference-based limitations on simultaneous communications within the WSN. The MAC protocol has to be adaptive in these different possible situations.

Taking these characteristics of wireless sensor networks into account, we focus our research in this thesis on designing a new MAC layer protocol by exploiting frequency offset based TR modulation in the physical layer. In designing the MAC protocol for WSN, we focus on achieving energy-efficiency with robust and adaptive communication with frequency offset based multi-channel medium access control. 


\subsection{Research objectives}

The main focus of this thesis is on providing energy-efficient connectivity with multiple access control in the MAC layer customized for transmit reference modulation in the underlying physical layer. The medium access control protocol needs to satisfy the following requirements: low-power, robust network-wide connectivity, multiple access control, traffic-adaptive behavior, and several other challenges, as mentioned in Section 1.2.

The general research question to be addressed in this thesis is:

How can the features of transmit reference modulation be exploited in a medium access control protocol for traffic-adaptive information dissemination with robust multiple access control in wireless sensor network, optimized for this radio technology in an energy-efficient manner?

The medium access control protocol has to deal with connectivity by providing working communication links in a duty-cycled wireless sensor network, where nodes are allowed to sleep most of the time. Connectivity here also includes multi-channel multiple access control using multiple frequency offsets of transmit reference modulation. Very low-power energy-efficient communication is the most important feature imposed by application requirements. Furthermore, the traffic-adaptive behavior here relates to a better quality of service performance of the protocol.

We address a number of open research challenges in the field of MAC protocols for wireless sensor networks mentioned in Section 1.2. In this section, we formulate a number of specific research questions regarding these research challenges. The rest of this thesis will focus on answering these research questions.

i) Research question 1: How can the features of transmit reference modulation be exploited in the MAC layer for WSNs?

ii) Research question 2: How can transmit reference modulation be used to achieve energy-efficiency in WSNs?

iii) Research question 3: How can traffic-adaptive behavior be attained in 
WSNs while maintaining energy-efficiency?

iv) Research question 4: How do the multi-channel properties affect the performance of multiple access control in a system using transmit reference modulation with frequency offsets?

v) Research question 5: How can an energy-efficient MAC protocol for WSNs be designed using transmit reference modulation for multi-channel medium access control using frequency offsets?

\subsection{Contributions and thesis organization}

In this section, we provide a short summary of the thesis organization. Different chapters of this thesis answers the individual research questions and contribute towards the main goal of the thesis. Therefore, in this section we explain the arrangement of the chapters by providing which research questions they answer. This thesis spans seven chapters. This chapter, Chapter 1, provides a brief introduction to the research areas covered in this thesis, presents our motivation, specifies the research challenges, formulates the research questions, and finally summarizes the contributions of this thesis. The research area of this thesis is medium access control protocol for wireless sensor networks. Our motivation to design a new MAC protocol for WSN comes from the numerous possible applications of the wireless sensor networks using an unique modulation technique of frequency offset based transmit reference modulation as the underlying physical layer. Hence, we present the research challenges, and formulate the research questions in the line of the research and design challenges in this chapter.

Chapter 2 presents the background and an elaborated literature review of existing WSN MAC layer protocols from the energy-efficiency perspective. Energyefficiency is one of the most important constraints in the energy-constrained low-power WSNs. Furthermore, the underlying frequency offset based transmit reference modulation has a performance penalty in the transmitter side because of transmitting both the modulated and unmodulated signal, and in the receiver side because of the self-mixing of these signals as well as the interfering signals 
of multiple frequency offsets using the same spectrum. As a result, the upper MAC layer has to contribute to improve the energy-efficiency of the sensor node and the operation of the wireless sensor network.

Chapter 3 answers the research questions 1 and 2 by proposing a new energyefficient MAC protocol for the WSN by exploiting the features of the underlying frequency offset based transmit reference modulation by minimizing its drawbacks. This chapter presents analytical modelling of this new MAC layer protocol and provides optimization techniques to improve the energy-efficiency. The new MAC layer protocol in Chapter 3 has been published on the following three publications $[19,20,21]$.

Chapter 4 answers the research question 3, where a traffic-adaptive algorithm has been proposed to disseminate higher number of packets in the WSN originated from an event-driven scenario. The important contribution of this approach is that the traffic adaptation is achieved while maintaining the energyefficiency of the MAC protocol. This chapter is based on the publication [22].

Chapter 5 answers research question 4 by providing a model to utilize the multichannel properties of the frequency offset based transmit reference modulation used in the underlying physical layer. The upper MAC layer can benefit from the possibility of multiple simultaneous transmissions using different frequency offsets offered by the transmit reference modulation. However, this excellent opportunity comes with certain limiting factors of a finite number of available frequency offsets, and a limitation on the total number of simultaneous transmitting frequency offsets. Hence, in this chapter we model the effects of frequency offset based TR modulation as a basic multiple access technique to analyze the potential benefits as well as to understand the consequences of the limiting factors at the upper MAC layer. The content of this chapter is based on the publication [23].

Chapter 6 answers research question 5, where the multi-channel properties of the frequency offset based TR modulation have been incorporated with the energy-efficient medium access control protocol for WSN. This chapter presents a detailed design of the MAC protocol from the multiple access perspective, and analyzes its consequence on the system capacity. This chapter builds 
upon the content of the Chapter 3 for the newly proposed energy-efficient MAC protocol, and also upon the contents of Chapter 5 for the multi-channel properties of frequency offset based TR modulation. In this chapter, we also present a complete finite state diagram for the protocol operation in a multiple access scenario.

Finally, Chapter 7 concludes this thesis, where we summarize our contributions, answer the research questions, provide conclusions on our presented work, and sketch the directions for the future work. 


\section{Background and Related Work}

This chapter discusses the most significant background and related work towards the contributions of this thesis for answering the research questions presented in Chapter 1. The discussion presented here represents the state-ofthe-art for the contents in the subsequent chapters of this thesis.

In this chapter, we discuss the importance of MAC protocols for WSNs in Section 2.1 from the perspective of energy-efficiency, traffic-adaptive behavior, and multiple access control. Afterwards, the transmit reference modulation technique is presented in Section 2.2 with its working principle as well as its impact on the MAC layer. Following this, a classification of existing most well known MAC protocols for wireless sensor networks is given in Section 2.3 with their advantages and drawbacks. We provide background information on MAC protocols from application requirements point of view, and review them from different protocol perspectives together with requirements of WSN. Subsequently, we present a classification of asynchronous preamble sampling protocols in Section 2.4 with a detailed analysis of many enhancements on the basic preamble sampling protocols. Finally, we conclude this chapter with summary in Section 2.5 .

\subsection{Importance of MAC protocols in WSNs}

The MAC protocol coordinates the transmission of packets to a shared communication medium, and determines when the participating nodes of the WSN 
are allowed to transmit or receive. A WSN is characterized with low cost, low memory, and low power sensor nodes with low computational capabilities. The main task of a sensor node in such networks is to sense, and to communicate that information via the wireless medium. Since the wireless channel is a shared transmission medium, any other node within the radio coverage area of the transmitter will be able to receive the packetized message. In such case, the MAC layer is responsible for successful transmission and reception of the message by controlling simultaneous communications in the common wireless medium.

A WSN is highly dependent on its application requirements. The network dynamics can also change quite quickly. This has a significant effect on the working principle of a MAC protocol. Moreover, the communication pattern varies from time to time depending on the occurrence of an event, based on a query, otherwise remains periodic. Furthermore, the MAC layer has to deal with retransmissions, if any packet is lost due to a collision with another message from any other sources. All in all, the key objective of the MAC layer is to organize and maintain the shared communication medium reliably with an efficient multiple access mechanism.

In this section, we present the importance of MAC protocols in WSNs from the perspective of energy-efficiency, traffic-adaptive behavior, and multiple access control; since these perspectives are within the scope of this thesis.

\subsubsection{Energy-efficiency}

WSNs have constraints on energy resources because of their typical deployment, which is often dense, distant, and difficult to access. Most often, the energy source is limited in such sensor networks that directly affect the maximum network lifetime. Since a MAC protocol directly controls the most energy consuming activities of transmission and reception of a wireless sensor node, a MAC protocol has to reduce the energy consumption for the protocol operation. An energy-efficient MAC protocol needs to reduce the major sources of energy waste such as idle listening, control packet overhead, overhearing, and collision; as identified by Ye [24] in the context WSNs. 
i. Idle listening: The major source of energy wastage according to [24] is idle listening. This occurs when a node spends energy while expecting to receive a packet, but does not receive any. The energy spent waiting to receive a packet is similar to the energy spent to actually receive a packet. Therefore, a node spends energy in idle listening while waiting to receive a potential packet, if the packet receiving time is not known, or not predictable by the receiver node. This is considered as the major source of inefficiency, because of the inherent low data rate nature of WSNs.

ii. Control packet overhead: Some protocols require network-wide exchange of control information to manage the active time of the nodes of a network, for example, a reservation of network resources or, a common wake up time. Such a strategy contributes to addition control packet or information exchange in WSNs. Thus the energy consumption increases to communicate the control information, and the complexity increases to maintain this schedule in the network.

iii. Overhearing: This occurs when one node redundantly receives a packet that is not destined for it. As a result, the node wastes energy by receiving the redundant packet that has to be deleted. Overhearing happens when all receiver nodes in receiving mode at a particular moment in the range of the transmitter node receive the transmitted packet, since the wireless medium by definition is broadcast in nature.

iv. Collision: A packet has to be discarded if colliding with another packet during transmission corrupts it. A collision causes the WSN to waste energy in both the receiver's and the transmitter's side. A receiver wastes energy by receiving a corrupted packet that has to be discarded eventually. In addition, the two or more transmitters involved have to perform retransmissions of the same packets once more. The potential receiver needs to be awake at the time of the transmission and the possible retransmission; otherwise the transmitter spends extra energy. This increases overall energy consumption. Presence of hidden terminals significantly increases this phenomenon, since the potential transmitters are out of the reach of each other. 


\subsubsection{Traffic-adaptive behavior}

When studying the behavior of MAC protocols, it is important to identify the kind of traffic they have to handle. Since MAC protocols in WSNs are generally heavily dependent on applications, two traffic patterns can be distinguished: periodic traffic, and event-driven variable rate traffic [3]. The periodic traffic includes processing and aggregation of data within a network of nodes at a regular interval. This accounts for most of the traffic in monitoring applications. This periodic traffic also includes the query based traffic, where nodes are asked to provide their data. However, these monitoring applications often encounter sudden event-driven variable rate traffic within the network after the occurrence of an event. In this case, the nodes require faster dissemination of the data to other nodes, because all data has to be communicated within a designated time to ensure their validity. Most energy-efficient MAC protocols achieve energy-efficiency by sacrificing performance for the QoS parameters [25]. The QoS parameters include lower delay, higher throughput, and lower latency. Hence, there exists a trade-off between better performance of QoS parameters and achieving energy-efficiency. Therefore, accommodating traffic-adaptive behavior is one of the key requirements of a MAC protocol to provide better QoS performance while maintaining energy-efficiency.

\subsubsection{Multiple access control}

The MAC layer is responsible for the channel access mechanism of the shared communication medium for multiple communicating nodes. WSNs mostly use single channel systems for operation. However, these single channel systems suffer throughput and capacity performance with the prevalence of Internet of Things (IoT) compared to a multi-channel system [26]. TR modulation offers a simple multi-channel capability through the use of multiple frequency offsets for simultaneous communications within the network. Although multiple transmissions can take place using several different frequency offsets destined to different receivers, the total interference level increases for a receiver. This happens due to the simultaneous communications, and cross-mixing among 
signals from different active nodes using different frequency offsets. As a result, the communication link could collapse at some point when the received signal is above the tolerable interference limit of a receiver. Therefore, the MAC layer requires a multiple access scheme such that the maximum allowable simultaneous communication limit is taken into account for a TR modulationbased system with multiple frequency offsets.

\subsection{Transmit reference modulation}

The physical layer underneath the MAC layer deals with the modulation of the data signal to the reference signal in a node of a WSN. The low-power sensor network has to deploy an efficient medium access control protocol together with an efficient modulation technique in the underlying physical layer to enable low power operation. TR modulation is a low-power and low data rate spreadspectrum modulation, which was introduced by Hoctor and Tomlinson [10] as a promising short range communication technique.

\subsubsection{Basic operation}

In the novel TR modulation operation in the physical layer, the transmitter sends both the modulated and the unmodulated signals with a known time or frequency offset. Initially, time offset based TR modulation has been re-

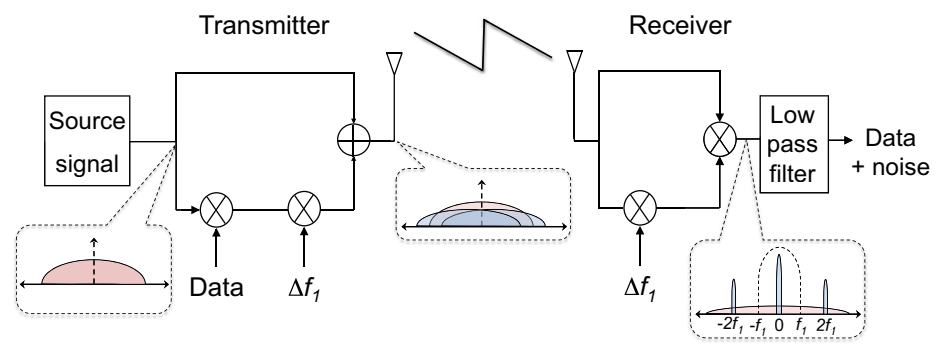

Figure 2.1: Transmit reference modulation 
searched in the context of ultra-wideband (UWB) systems [10, 11, 12], which offers higher transmission bandwidths. Hence, the receiver gets resistance to the multi-path fading effect, since higher transmission bandwidth leads to higher channel resolution for the receiver [15]. The capability of resistance to multi-path fading makes TR modulation suitable for robust communication in the harsh application environments of WSNs. Afterwards, a frequency offset based TR modulation has been researched in [7, 13, 14], as time offset is difficult to realize on a small chip because of the associated wideband delay element. Furthermore, a frequency offset based TR modulation provides performance improvements with a simple receiver architecture compared to time offset based TR modulation [13].

The working mechanism of TR modulation is presented in Figure 2.1, where we see the transmitter in the left side, and the receiver in the right side. In the transmitter, the source signal is divided into two components, one without modulation - known as unmodulated reference signal; and one with modulation with data and a frequency offset - known as modulated signal. Later on, these two components are combined, and sent through the channel. The reference signal and modulated signal in the channel are visibly separated by the frequency offset, as can be seen in the Figure 2.1.

Following the transmission, a receiver performs demodulation by correlating the received signal with a frequency shifted version of itself, without using rake receiver or channel estimation. The demodulation with simple self-correlation becomes possible, since the reference signal is also available in the received signal, and all multi-path components contain identically distorted signals. This interesting property of TR modulation allows the use pseudo-random noise as information carrier that is also easy to generate [8]. Finally, the demodulation restores the original data after passing through a low-pass detection filter. This receiver implementation reduces the complexity of the receiver, achieves faster synchronization, and results in energy-efficient receiver implementation. 


\subsubsection{Impact of TR Modulation on the MAC Layer}

The MAC protocol in WSNs is responsible for creating a network infrastructure with addressing, and providing a channel access mechanism for network nodes to effectively communicate in a shared wireless communication medium. However, minimizing energy consumption in WSNs is always a big challenge in the MAC layer. This problem maximizes for a modulation technique like TR modulation, which takes more energy burden in the transmitter side by transmitting the reference signal together with the modulated signal. Conversely, the TR modulation enabled receiver enjoys some possibilities to reduce the power consumption.

Interestingly, TR modulation provides many lucrative advantages for the receiver, and all together offers many opportunities to be exploited in the upper MAC layer [15]. Firstly, the receiver can restore the original signal quickly by simple self-correlation with the frequency shifted multi-path components of the same signal. As a result, the receiver with TR modulation requires small signal acquisition time. Secondly, the receiver with TR modulation enjoys faster synchronization time to detect and decode the received signal, which is highly desirable for low duty cycle transmissions for WSNs. Thirdly, the receiver becomes energy-efficient, since it does not require a complex rake receiver technique, channel state information, or power-hungry stable oscillator to restore the original signal. Fourthly, the TR modulation allows multiple nodes to transmit simultaneously and asynchronously using multiple frequency offsets without any mutual timing coordination. Thus, such a system provides inherent capabilities for multiple access, which needs to be exploited in the medium access control layer to coordinate the shared of use the wireless medium within the WSN.

Together with the opportunities, the TR modulation provides some challenges to be explored in the MAC layer. The TR modulation consumes higher power at the transmitter side by sending both the modulated and unmodulated signal in the channel [7]. Furthermore, the use of a large number of active links with multiple frequency offsets increases the overall noise-floor at the receiver side. The self-mixing of the modulated and unmodulated signal also contributes to 
the increase of the noise-floor at the receiver, which contributes to the overall performance penalty $[87,17,15]$.

TR modulation can be a potential candidate for asynchronous low power and low data rate communication in wireless sensor networks offering interesting features and flexibilities to the upper MAC layer. Furthermore, special MAC features have to be introduced to deal with the performance penalty of the frequency offset TR modulation while exploiting its special opportunities.

\subsection{Existing MAC protocols for WSNs}

A large number of MAC protocols have been proposed in the last decade addressing different applications and requirements of WSNs. Different medium access mechanisms have been applied with possible combinations of wireless channel access techniques. Many researchers have surveyed MAC protocols in WSNs from different contexts in the past $[3,5,6,27,28,29,30]$. Since TR modulation has a power penalty at both the transmitter and the receiver side, we focus on energy efficiency of MAC protocols for achieving multiple access control, and traffic-adaptive behavior for our research. In this section, we classify the existing MAC protocols in five categories in the following subsections, and eventually focus on the most energy-efficient category of MAC protocols.

\subsubsection{Reservation-based scheduled protocols}

In reservation-based scheduled protocols, certain network resources are reserved for each sensor node in which it is allowed to communicate with other nodes of the network. A node has to wait for its resource to send data. Hence, a network-wide dissemination of information is required in this type of MAC protocols to establish a schedule for multiple nodes to access the channel. These schedules are established based on different requirements; such as reducing collisions, increasing throughputs, minimizing delays, and ensuring fairness among nodes. Different multiplexing techniques such as time division multiple access (TDMA), frequency division multiple access (FDMA), code division mul- 
tiple access (CDMA), space division multiple access (SDMA) are representative examples of this category. Figure 2.2(a) represents the basic timing diagram of TDMA or TDMA-like MAC protocols [31, 32, 33, 34], which are good examples of this reservation based scheduled protocols category.

In TDMA or TDMA-like protocol, each sensor node is assigned a slot to transmit or receive, which is part of a frame. Therefore, the nodes send and receive in their specific slots, and can sleep the rest of the time. However, a node needs to know the exact time slot when its neighbor will be awake before transmitting to it. Dissemination of this slot information requires networkwide communication, which significantly increases the control packet overhead. Inherently, this technique allows collision reduction, and increases throughput for a high data rate network. However, this achievement comes at the cost of creating slots, and synchronizing all the sensor nodes of the network to their specific slots. Hence, this technique is suitable for periodic traffic with high data rate, but is not suitable for energy-efficient low data rate application scenarios in WSN.

\subsubsection{Synchronous MAC Protocols}

Synchronous MAC protocols establish a common active period in the network to wake up to actively communicate among nodes, and allow the nodes to sleep for the rest of the time. Therefore, synchronizing active period among the neighboring nodes is an extra task that needs to be performed in this category of MAC protocols. However, this synchronization of active periods in this category of MAC protocols are not as stringent as in reservation-based scheduled TDMAlike protocols. In synchronous MAC protocols, one node listens to the channel for some time for any activity. If it does not receive any schedule information during the active time, then it determines its own schedule to wake up for the next time, and broadcast it before going to sleep. Thus, this node acts as a synchronizer. Another node receiving a schedule from its neighboring nodes follows the receiving schedule over its own schedule. Thus, the second node becomes a follower to the synchronizer node. In this way, a cluster of nodes becomes synchronized to a synchronizer node. If one node receives a new 
schedule after it sets its own schedule, then it also adds the new schedule. In this way, this node can act as a bridge node between two clusters by waking up frequently. The most common protocols with common active period are S-MAC [35], T-MAC [36], RMAC [37].

Figure 2.2(b) represents the basic timing diagram for protocols with common active period. A node has to wait for the next active period to send data. Thus, the contention period to access the shared medium is reduced to the active period. This category also requires network-wide synchronization of the active period for all the participating nodes, which is expensive from energy-efficiency perspective. As a result, the synchronous MAC protocols with common active periods are most suitable for periodic traffic. However, this category of protocol is not energy efficient for irregular event-driven traffic, which is very common in WSNs.

\subsubsection{Asynchronous preamble sampling protocols}

Asynchronous preamble sampling protocols enable a node in a WSN to spend in low-power sleep mode for most of the time, and wake up for a short time to sample the channel for activity detection. This is known as duty cycling, and this technique is widely adopted in WSN for its energy saving capability [2]. Each node of the network can asynchronously choose its duty cycle time independent of all other nodes in the network. However, the nodes in the WSN can communicate only when they are active. Therefore, to enable successful communication, the transmitter has to precede the data packet with a preamble packet that is long enough to be detected by all potential receivers. The working principle of asynchronous preamble sampling protocol is illustrated in the timing diagram in Figure 2.2(c).

The duration between two consecutive duty cycle of a node is known as channel sampling interval or check interval. The preamble length has to be as long as the check interval to make sure that all potential receivers are awake during preamble transmission. If the receiver detects some part of the preamble during its sampling time, then it continues to listen to the channel to receive the data packet from the transmitter; otherwise returns back to sleep. If any overhearer 
listens to the preamble, then it has to continue to listen till the end of the preamble before receiving the data packet. The overhearer eventually discards the data packet. This category is also known as low power listening (LPL) protocols [38]. Most common preamble sampling protocols are BMAC [38], X-MAC [39], and WiseMAC [40].

Asynchronous preamble sampling protocols are very suitable for low data rate asynchronous applications; since this strategy enables the nodes to be energy-efficient without any network-wide synchronization and management. However, in the basic preamble sampling protocol, one node occupies the channel by sending preambles as long as the check interval of the potential receiver, and prevents other nodes to access the channel. Thus, the achievable throughput is limited for basic preamble sampling protocols. Therefore, these asynchronous preamble sampling protocols employ various techniques to increase throughput. Another drawback of preamble sampling protocol is that the overhearers waste energy by listening to the complete preamble before receiving the actual data packet. However, the benefit of preamble sampling protocol is that the preamble duration can be made shorter by reducing the check interval of the nodes. In this way, the preamble sampling protocols can save the overhearing energy, and can achieve lower packet delay delay with ultra-low duty cycle.

For preamble sampling protocols, the transmitter takes the burden of delivering the packet to the receiver, since the receiver sleeps most of the time. Also, the receiver and the overhearers need to receive the preamble first before receiving the packet. Although this technique introduces asymmetric energy consumption, the overall energy consumption for this category of MAC protocols remains minimal, when compared to other categories $[2,6]$. Receiver side may carry the burden by sampling the channel frequently, which allows the transmitter to minimize the preamble duration as it depends on the duration of two consecutive channel sampling intervals of the receiver. In these protocols, the idle listening is reduced to possible minimum, and network-wide synchronization is not necessary; thus this category of MAC protocols are the most energy saving. 


\subsubsection{Hybrid protocols}

The hybrid protocols combine different characteristics of the previously mentioned categories to enhance the protocol performance. For example, Funneling MAC [41] is a hybrid protocol that uses spatial separation by using TDMA close to the sink node to accommodate relatively high traffic load, but uses carrier sense multiple access (CSMA) for the rest of the network. Another hybrid protocol Zebra MAC (ZMAC) [42] uses a similar technique by using CSMA when the traffic load is low, and switches to TDMA when the traffic load is high. Scheduled channel polling (SCP) [43] is another hybrid protocol that uses a common active period to communicate, but allows the nodes to go back to sleep in the absence of packets in the contention period, like the preamble sampling protocols.

Depending on different requirements, ZigBee [44] is a flexible hybrid MAC that can switch to different modes, e.g., presence or absence of beacon, TDMA-like operation, or carrier sense multiple access/collision avoidance (CSMA/CA)-like operation. The ZigBee standard also has a power saving mode; where an access point with unlimited power will store the traffic for sensor nodes, and send beacons with traffic indication map. Once a sensor node wakes up and receives a beacon, it can poll to the access point to receive the data. However, this will not be suitable in the absence of an access point, which is sometimes the case for a WSN.

The hybrid protocols available in the literature focus on maximizing some performance parameters for WSNs, but most of them are not energy-efficient. Furthermore, some hybrid protocols accommodate different modes of operation for different traffic loads without emphasizing on energy saving.

\subsubsection{MAC protocols with wake up radios}

The MAC protocols having wake up radios add another radio to the node architecture. The purpose of this wake-up radio is to detect the communication, then perform a fast, on demand wake up to the sleeping main receiver; which sleeps otherwise. If the wake up radio is sensitive, then it will falsely wake up 
the main receiver that will drain energy. Moreover, the wake up radio has to be turned on all the time to detect a communication, which is energy inefficient. Also the total cost of the node will increase, if an additional wake up radio is added. An example protocol that uses wake up radio is STEM [45].

\subsubsection{Summary}

In this section, the existing MAC protocols for WSN are classified into five categories: i) reservation based, ii) synchronous, iii) asynchronous preamble sampling, iv) hybrid, v) protocols with wake up radios. The aim of this classification is to analyze the categories of MAC protocols in line with the requirements and characteristics of the WSN employing the TR modulation in the underlying physical layer. Therefore, achieving energy-efficiency is considered the most important parameter, since the TR modulation has relatively high power penalty at both the transmitter and the receiver side. The MAC protocol categories are analyzed to identify the category that minimizes the major sources of energy waste, namely: i) idle listening, ii) control packet overhead, iii) overhearing, and iv) collision.

Among the MAC protocol categories, the asynchronous preamble sampling protocols provide the most appealing features for WSNs using TR modulation. Compared to other categories of protocols, the preamble sampling protocols consumes less energy by minimizing idle listening with low duty cycles for the nodes. The control packet overhead is minimized for this category of protocols, since no common schedule is required to be created, exchanged or maintained. For low data rate traffic, this asynchronous duty cycling mechanism saves energy, and minimizes overhearing and collision because of the extra information exchange to communicate the synchronized wake up time over the network. Therefore, the asynchronous preamble sampling protocols are the most suitable for WSNs with low power, low complexity, and low data rate requirement. 

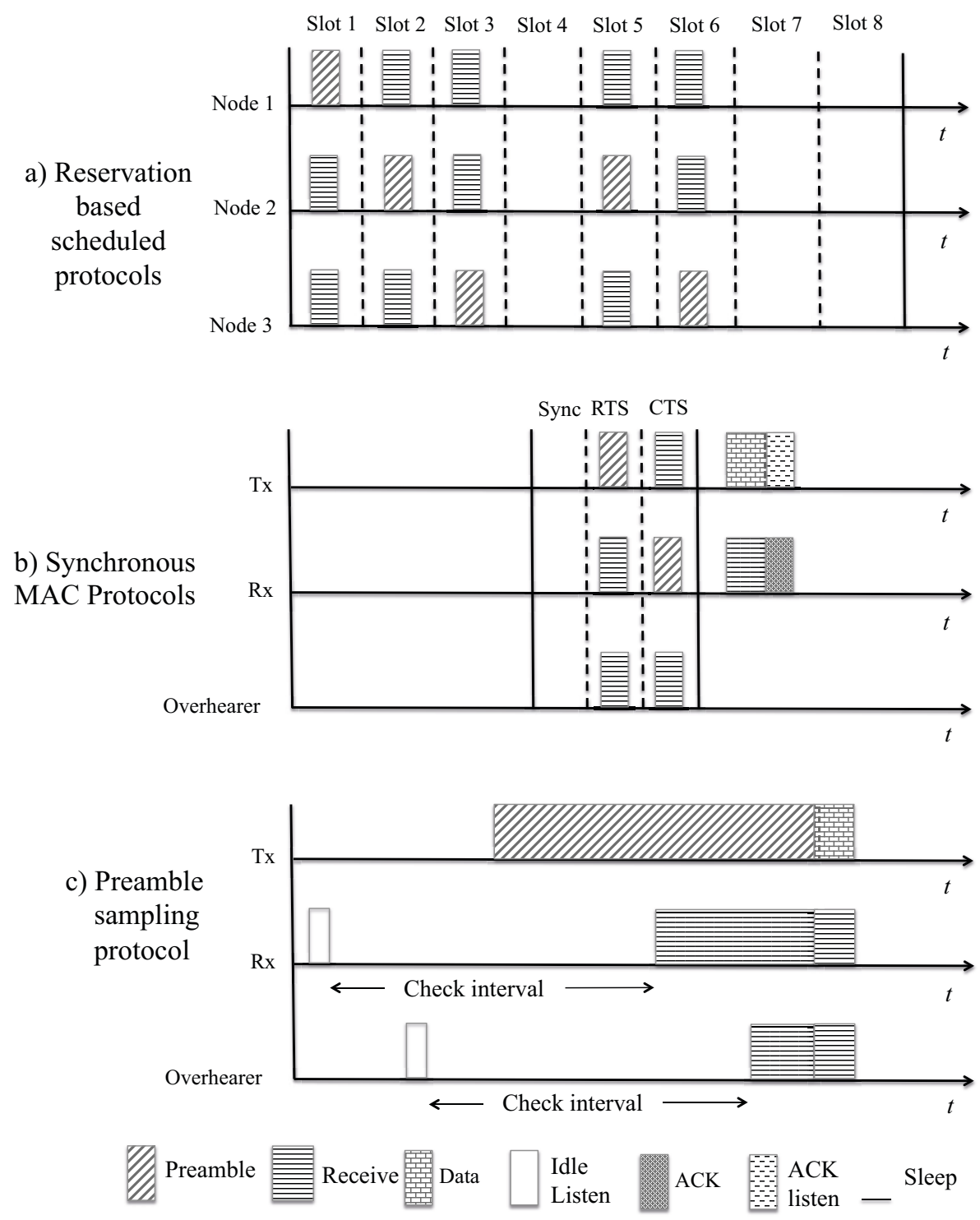

Figure 2.2: Classification of MAC protocols 


\subsection{Classification of asynchronous preamble sampling protocols}

Section 2.3 establishes preamble sampling protocols as the most energy-efficient category of MAC protocols. Thus we explore this category of MAC protocols further, since it is related to our research interest.

\subsubsection{Basic preamble sampling protocols}

In the basic preamble sampling protocols, the nodes switches off their transceiver to sleep mode, and periodically samples the channel according to their duty cycle. This periodic duty cycle can be performed without any network-wide information exchange among the nodes of the network. In this way, the nodes save energy in preamble sampling protocols. The duration between two consecutive channel sampling of a node is known as the check interval. To communicate successfully, the transmitter node sends preamble of duration at least as long as the check interval before sending the data packet. Therefore, the intended receiver node can receive the preamble whenever it wakes up, assuming all the nodes have the same check interval. After detecting the preamble, the receiver node continues to listen till the end of the preamble, and then receives the data packet. The overhearer node also performs the same procedure as the intended receiver. The working principle of a basic preamble sampling protocol is illustrated in Figure 2.3(a). The preamble sampling protocols are also referred to as low power listening protocols. B-MAC [38] and WiseMAC [40] are the examples of basic preamble sampling protocols.

The basic preamble sampling protocol wastes energy in overhearing by using the long preamble. Furthermore, the channel is occupied by one node by sending a preamble as long as the check interval duration of the receiver. That is why, the preamble sampling protocols try to reduce the preamble duration to save energy by making a node to wake up frequently reducing its check interval. This also minimizes the energy waste of the overhearers, and achieves satisfactory delay while maintaining low duty cycle. Researchers have analyzed 
the preamble sampling protocols to reduce the preamble duration mainly in three ways: i) protocols with preamble packetization, ii) schedule learning, and ii) adaptive duty cycles [6]. We discuss these techniques in detail in the subsequent subsections.

\subsubsection{Protocols with preamble packetization}

The protocols with packetization enable the transmitter to replace the long preamble by short preamble packet bursts with a destination address. Hence, receiving a single preamble packet allows the target receiver to continue listening to receive the data, and send an acknowledgement afterwards. The overhearers can go back to sleep after receiving only one small preamble packet, instead of listening to the large preamble using the basic preamble sampling method. In this way, the MAC protocols with preamble packetization achieve energy saving in overhearing. However, the maximum preamble length remains the same for all the future communications, because this category of MAC protocols does not adapt the preamble length for future communications with the same node.

In another alternative implementation of protocols with preamble packetization, the transmitter sends a short preamble packet, then listens for the acknowledgment of this short preamble from the receiver. The transmitter has to repeat this cycle of sending short preambles and waiting for the acknowledgement for at least the check interval duration. By waiting for the acknowledgement after sending the preamble, the transmitter is able to shorten its preamble length by receiving the acknowledgement from the intended receiver. The overhearers return to sleep after receiving one short preamble without sending an acknowledgement. This category of MAC protocols lacks the acknowledgement after successful data transmission, since the acknowledgement is used to indicate successful preamble reception.

Several preamble sampling MAC protocols exists in the literature that perform packetization of preamble or data. They can be subdivided into the following approaches: i) preambles with destination address, ii) preambles with data start information, iii) preambles with acknowledgement. Figure 2.3 represents these 

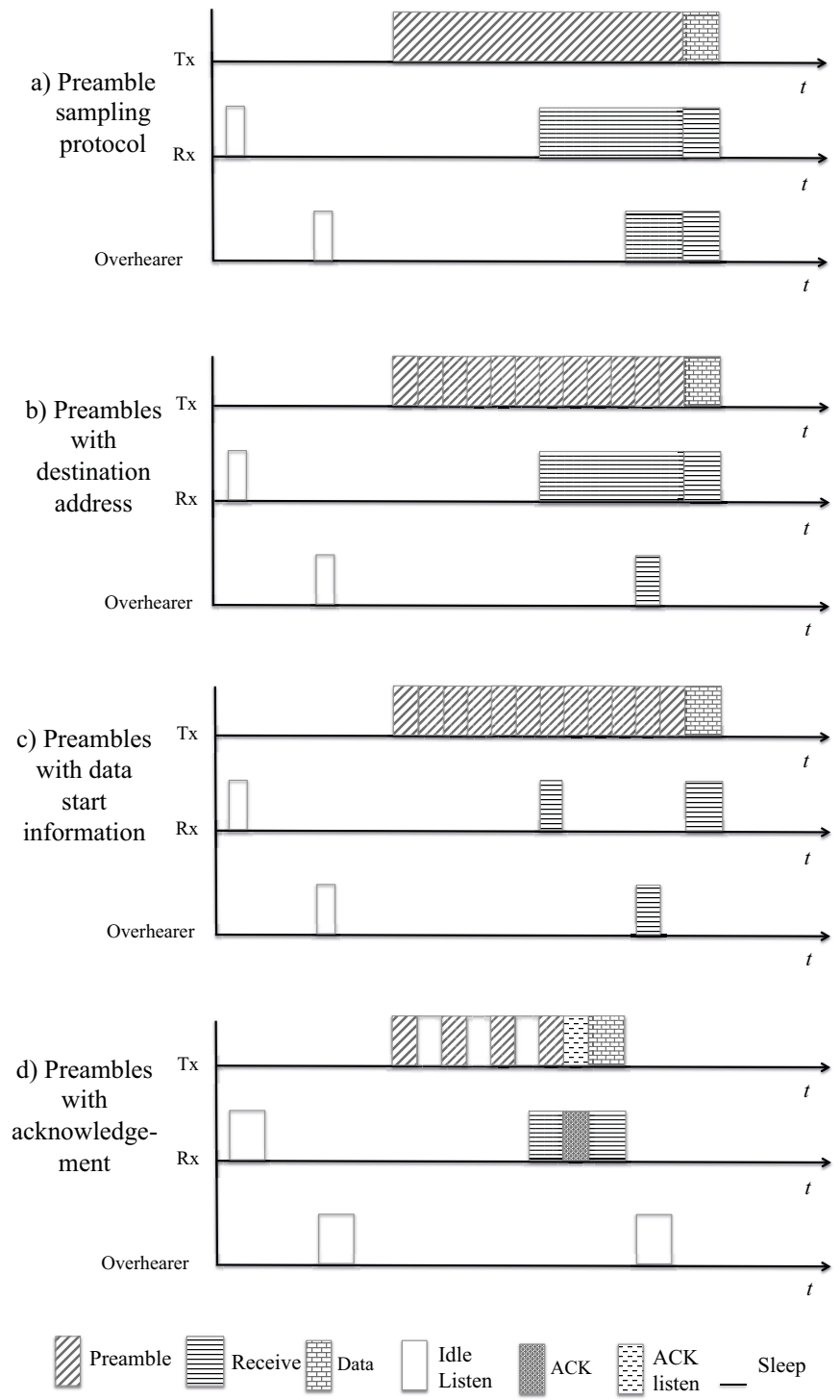

Figure 2.3: Classification of MAC protocols with packetization 
different categories of preamble sampling protocols.

\subsubsection{Preambles with destination address}

Some preamble sampling MAC protocols split the long preamble packet into many short ones with the destination address built into them. As a result, the overhearers can return to sleep after receiving only one short preamble. Hence, the overhearer will not waste any extra energy by receiving the whole preamble only to find out later that it was not the intended receiver. ENB-MAC [46] is an example of such kind of protocols, as illustrated in Figure 2.3(b).

\subsubsection{Preambles with data start information}

This category of MAC protocols send repetitions of packetized preambles with information about the start of data transmission together with the destination address. When the intended receiver wakes up during its periodic listening and receives one of these preambles, the receiver returns to sleep and wakes up again when the data transmission starts. This mechanism is illustrated in Figure 2.3(c). Examples of these protocols include B-MAC+ [46], Speck-MAC-B [47], DPS-MAC [48], SESP-MAC [49]. Sometimes protocols send repetitions of data packets with useful information and destination address instead of sending preambles, but do not request any acknowledgement. Then the receiver can return to sleep after receiving one small data packet. SpeckMAC-D [47] is an example of such protocols.

\subsubsection{Preambles with acknowledgement}

One interesting category of MAC protocols enables the transmitter to send repetitions of short preamble packets followed by an acknowledgement receiving period. As a result, the receiver can send an acknowledgement right after receiving the preamble. Hence, the transmitter can send the data right away to the destination node. This technique saves energy in both the transmitter and the receiver sides. The transmitter can effectively shorten its preamble and acknowledgement iterations, and the receiver can return to sleep early without 
the need to wake up once again. This technique is presented in Figure 2.3(d), and example protocols include CSMA-MPS [50], X-MAC [39], ContikiMAC [51], AREA-MAC [52], TICER [53].

\subsubsection{Schedule learning}

Some preamble sampling MAC protocols take the basic preamble sampling technique to operate on, but in addition add a state using receiver-driven communication strategy; where the transmitter adapts the start of the preamble by remembering the receiver's next periodic wake up time. Therefore, this category of protocols are essentially receiver-driven protocols that enable the transmitter to minimize its preamble length after the first time communication.

\subsubsection{Remembering receiver's next wake up time}

This category of MAC protocols remembers the next wake up time of a receiver after one communication. The first communication takes place with the actual longer default length preamble, but this technique allows the transmitter to shorten the preamble length of the future communications by targeting the next wake up time of the intended receiver. The transmitter learns about the receiver's next wake up time by the information sent along with the acknowledgement sent by the receiver. In this way, the transmitter becomes synchronized with the receiver for future communications. Figure 2.4(b) represents the timing diagram for the protocols with schedule learning. WiseMAC [40], CSMA-MPS [50], ContikiMAC [51], SyncWUF [54], and TrawMAC [55] are examples of this category.

Receiver-driven protocols are unsuitable for broadcast traffic, because one transmitter might have to adapt its preamble multiple times for its multiple neighbors. Furthermore, this category of protocols lacks a transmitter-driven strategy in the synchronized link state. Per packet overhead in low traffic increases the energy consumption for these protocols in the unsynchronized link state, since the potential receiver and all the overhearers have to listen the complete preamble before receiving the data packet. Moreover, the nodes have to store extra 


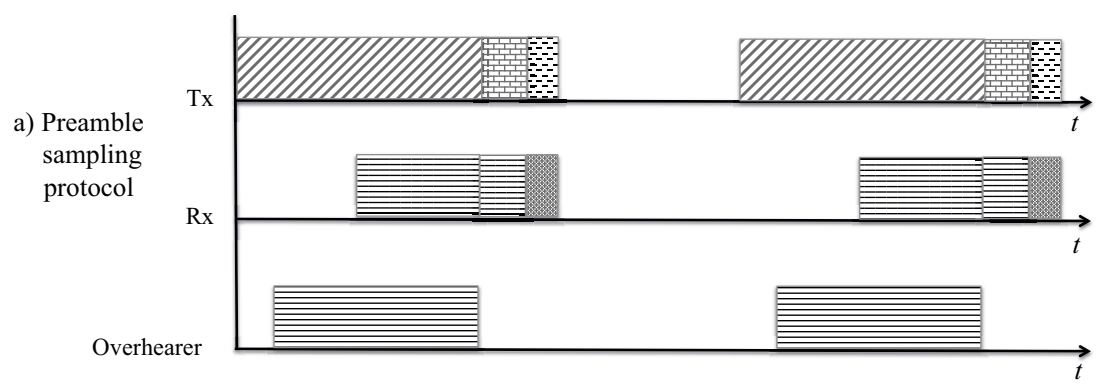

b) Remembering Receiver's next wake up time

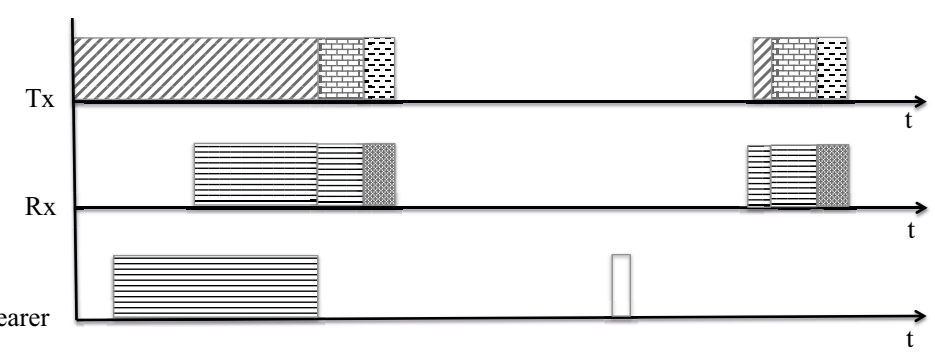

Overheare
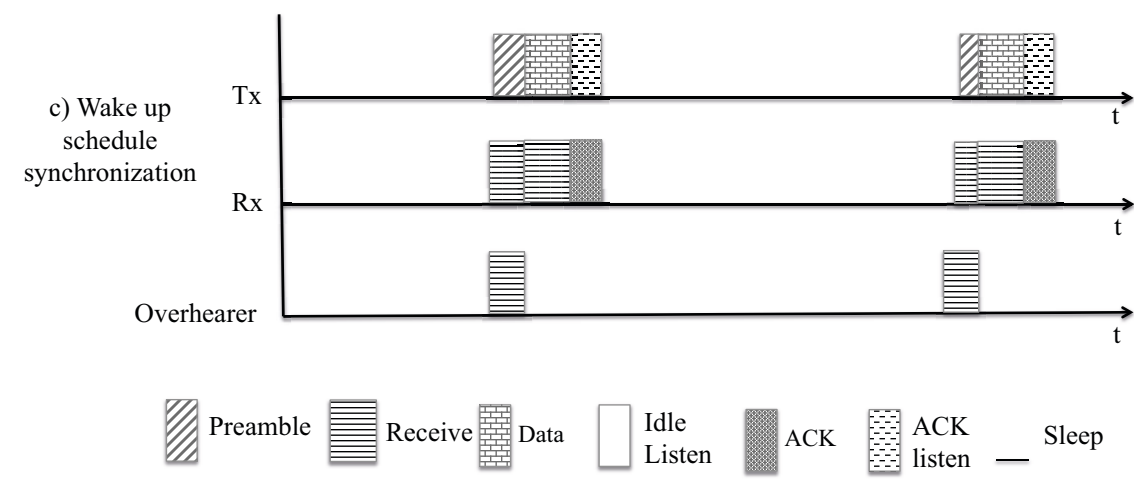

Figure 2.4: Classification of schedule learning MAC protocols

information of their respective neighbor node's channel sensing times that takes valuable storage space in a high density network. 


\subsubsection{Wake up schedule synchronization}

This group of MAC protocols synchronizes wake-up schedule to wake up at the same time while using a preamble sampling technique. These protocols work better with broadcast communications, and require less information to store; because of sharing the same wake up time. However, this advantage comes at the expense of network-wide control information dissemination to synchronize the wake-up times of all the participating nodes in the WSN. Figure 2.4(c) represents operation of such protocols. SCP-MAC [43] uses scheduled channel polling by synchronizing the wake up times of all the neighbor nodes. MiXMAC [56] synchronizes the wake up times of the nodes in a path to reduce the packet delivering delay from a particular source to a particular destination.

\subsubsection{Adaptive duty cycle}

This category of preamble sampling protocols are capable of adapting their duty cycle based on different requirements to enhance the protocol performance for the QoS parameters; for example, increasing throughput, decreasing per packet delay, ensuring utilization of available network resources to the maximum level. These requirements include topology information, requests from neighborhood, and variable data traffic. However, these duty cycle adaptive protocols do not adapt their network operation based on energy availability on individual nodes, often sacrifice energy-efficiency to achieve better QoS performance, and target only specific application scenarios.

\subsubsection{Request based}

Request based protocols adapt their duty cycles depending on the requests from the neighboring nodes. For example, one transmitter may use a single bit to request the receiver to keep listening to receive more packets after receiving one packet. For example, WiseMAC with more bit [40], and an extension of WiseMAC with stay awake promise [57] are such kind of protocols. BEAM [58] allows one node to double its duty cycle based on a specific request from the 
transmitter. AREA-MAC [52] allows nodes to adapt their duty cycle based on a specific request with a goal to minimize the delay from source to sink.

\subsubsection{Traffic load based}

This category of MAC protocols adapt their duty cycle based on the traffic. An extension of the B-MAC+ protocol in [59] proposes that the transmitter indicates the receiver before a burst packet transmission, so that the receiver can increase its duty cycle. MaxMAC [25] enables duty cycle adaptation based on varying traffic load, where the nodes wake up more frequently based on the high incoming traffic. SCP-MAC [43] adds extra duty cycles after receiving a packet, and releases these extra duty cycles when they are not useful.

\subsubsection{Topology information based}

Some protocols adjust their duty cycle based on the position of the node in a topology. EA-ALPL [60] performs the adaptation of duty cycle assuming a tree topology, where one node sets its own duty cycle depending on the number of descendants in the routing tree. Nodes learn their position in the tree based on the number of the forwarded messages. Preamble sampling with state information [61] protocol allows the nodes to adjust the duty cycle based on the position relative to the sink node. The nodes closer to the sink have higher duty cycles, whereas the nodes further away from the sink have lower duty cycles.

\subsubsection{Summary of preamble sampling protocols}

In this section, the preamble sampling protocols are analyzed in detail to have a further understanding of this category. The preamble sampling protocols are grouped into three categories, namely: i) protocols with preamble packetization, ii) schedule learning, and ii) adaptive duty cycles. Firstly, in the preamble packetization category, a significant amount of energy saving is achieved for overhearing by making the preambles into short bursts with extra information. Employing the early acknowledgment technique together with short preambles helps to minimize the preamble duration from the transmitter side. This 
category of preamble sampling protocols are suitable for high density WSN, because shortening the preamble is beneficial in this scenario. Secondly, learning the wake up schedule reduces the preamble length for the future communication with additional complexity of remembering the neighbor's future wake up time. Thus, this category saves energy for future communications with a smaller preamble, and is suitable for a low density WSN. Finally, the adaptive duty cycle MAC protocols are capable to adapt to varying traffic load while maintaining energy-efficiency using preamble sampling technique. As a result, this category is suitable to achieve better performance in the quality of service parameters in the presence of varying traffic.

\subsection{Summary}

Wireless sensor networks are characterized with energy-constrained low complexity network with low power sensor nodes distributed over an area to collect and communicate information using the shared wireless medium. As a result, the WSNs generally deal with low data rate communication, since the energy consumption increases linearly with the amount of information exchanged. That is why, the sensor nodes in WSN are frequently put to sleep mode to achieve energy-efficiency. The QoS parameters, like increasing throughput or decreasing delay, is achieved while maintaining energy-efficiency.

We observe that preamble sampling protocols provide flexibility with low power and low cost operation by enabling the nodes to have short channel sampling time without network-wide synchronization overhead. Compared to other MAC protocol categories, the preamble sampling protocols has less energy consumption due to the minimization of the idle listening. Hence, preamble sampling protocols are appealing for the requirements of WSNs, which requires energy-constrained sensor nodes to deal with low traffic load based applications. The basic preamble sampling technique is further used to enhance the energyefficiency by employing several techniques. Some preamble sampling protocols minimize the energy consumption using short bursts of preamble with extra information instead of a long one. Another few protocols use early acknowl- 
edgement together with the short bursts of preambles to minimize the preamble length to save energy. Thus, this category of preamble sampling protocols is suitable for dense WSNs. There is another category of preamble sampling protocols that exploit synchronization information of the nodes to make the preamble length shorter for future communications. Hence, these protocols are suitable for low density WSN. The traffic adaptive category of preamble sampling protocols adapt to the traffic behavior while maintaining energy-efficiency.

Using the transmit reference modulation in the underlying physical layer opens new prospects to be analyzed in the upper MAC layer. The transmit reference modulation provides smaller signal acquisition time, faster synchronization time to detect and decode the received signal, and different frequency offsets for multiple access. However, transmit reference modulation also comes with a power penalty because of transmitting the reference signal together with the modulated signal, and the cross-mixing of the signals at the receiver side. The existing MAC protocols are not customized to benefit from the opportunities provided by the transmit reference modulation technique in the underlying physical layer. Therefore, in the subsequent chapters of this thesis, we focus on preamble sampling protocols in the MAC layer to achieve energy-efficiency, traffic-adaptive behavior, and multiple access control using the opportunities provided by the transmit reference modulation in the physical layer in the context of wireless sensor networks. 


\section{CHAPTER 3}

\section{Energy-efficient medium access control}

The medium access control protocol determines the energy consumption of a wireless sensor node by specifying the listening, transmitting, or sleeping time. Therefore, MAC protocols play an important role in minimizing the overall energy consumption in a typical wireless sensor network. Using transmit reference modulation in the underlying physical layer opens up new possibilities and challenges to be investigated in the upper MAC layer. Hence, this chapter presents a new energy-efficient MAC protocol, called TR-MAC, to exploit all the benefits provided by the TR modulation in the physical layer while minimizing its drawbacks. TR-MAC enables both transmitter-driven and receiver-driven communication in the WSN, allows a pair of nodes to use individual frequency offsets for multiple access, and is capable of achieving fast synchronization in the receiver side to reduce energy consumption. In short, TR-MAC is an energy-driven MAC layer communication protocol for asynchronous low data rate applications that enables nodes to choose between transmitter-driven or receiver-driven communication possibilities based on the available energy in the node.

This chapter expands the answer to research question 1 ("How can the features of transmit reference modulation be exploited in the MAC layer of WSNs?"), and research question 2 ("How can transmit reference modulation be used to achieve energy-efficiency in WSNs?"), as mentioned in Chapter 1 . The contributions of this chapter comes from the following publications [19, 20, 21], and are as follows: (1) we introduce a MAC protocol, TR-MAC, exploiting the 
characteristics of TR modulation, and provide detailed design with models to analyze the energy consumption; (2) we provide an explicit optimization for the TR-MAC protocol depending on the experienced traffic load; (3) we provide a validation of the analytical models of the TR-MAC protocol by evaluating the energy consumption using simulated results obtained from the simulator; (4) we provide a comparison of TR-MAC protocol with other reference protocols, and show that the results of the comparison works favorably for TR-MAC protocol over similar reference protocols.

This chapter is organized as follows: Section 3.1 explains the motivation behind this research. The TR-MAC protocol design is presented in Section 3.2 with the basic protocol operation and corresponding states. Afterwards, the analytical modelling for TR-MAC is given in Section 3.3. Subsequently, the optimization model for the TR-MAC protocol is presented in Section 3.4. The comparison of TR-MAC protocol with relevant similar WSN protocols is elaborated in Section 3.5. We present the TR-MAC protocol implementation details in the OMNeT++ simulator, and compare the analytical results with simulation results in Section 3.6. Finally, we summarize this chapter in Section 3.7.

\subsection{Motivation}

Low-power wireless sensor devices have gained popularity in the past decade. These low-power devices have to deploy an efficient medium access control protocol together with an efficient modulation technique in the underlying physical layer to enable low power operation. TR modulation, as discussed in Chapter 1 and 2, is such a low-power spread-spectrum technique [10], where the transmitter sends the unmodulated carrier signal together with the modulated signal in the wireless medium separated by a frequency offset already known to the receiver. The receiver restores the original signal using the known frequency offset by performing self-correlation with the frequency shifted version of the same signal [8]. Hence, the receiver enjoys faster synchronization with reduced signal acquisition time without the need of channel estimation, or a complex rake receiver, or a power-hungry stable oscillator. Moreover, multiple frequency 
offsets using TR modulation can be used to provide multiple access to the common shared medium. As a result, the TR modulation with its simplified receiver architecture enabling low power operation provides flexibilities to the upper MAC layer for WSNs. However, TR modulation has a power penalty in the transmitter because of sending the reference signal together with the modulated signal, which accounts for $3 \mathrm{~dB}$ power penalty [7]. Furthermore, the cross-mixing of the received signal increases the overall noise-floor at the receiver, which further increases the overall power penalty in TR modulation to $10.7 \mathrm{~dB}[16,17]$. Therefore, an energy-efficient MAC layer protocol customized to the TR modulation is necessary to exploit all the advantages provided by the TR modulation, while minimizing its drawbacks.

\subsection{TR-MAC protocol: design and operation}

We propose a new energy-efficient protocol, TR-MAC, which exploits the opportunities provided by TR modulation in the underlying physical layer, and simultaneously mitigates the transmit power penalty of TR modulation. The TR-MAC protocol combines the best characteristics from all three categories of the energy-efficient preamble sampling protocols, which is discussed in Chapter 2. The transmitter using TR-MAC protocol packetizes the preamble with the destination address. The receiver using TR-MAC protocol can detect any transmission in the channel with a very short preamble, because the receiver architecture using TR modulation has fast signal acquisition and synchronization capability. As a result, TR-MAC protocol enables the transmitter to transmit very short preamble. To exploit this advantage of TR modulation further, small data packet can be appended with the preamble, as the data packets in wireless sensor networks are generally very small and within a range of few bytes. Therefore, the combination of preamble and data is referred to as preamble-data for the rest of this thesis.

The TR-MAC protocol is a preamble sampling protocol that saves energy in the node by allowing the transceiver to sleep most of the time. One node periodically wakes up for a short amount of time to receive a transmitted packet 
in the channel. This time duration to detect channel activity is known as periodic listening. The maximum duration between two consecutive periodic wake up times of a node is known as the check interval, and is globally known in the network. Since the transmitter does not know when the potential receiver node will wake up, it has to take the responsibility to reach the receiver during his periodic listening time. As a result, the transmitter uses this check interval as the maximum duration to transmit its preamble-data in order to communicate the message to the receiver when it is awake. To shorten the preamble length from from the maximum duration of the check interval, the transmitter transmits one preamble-data, then listens for a potential acknowledgement from the receiver, and continues this cycle until it receives an acknowledgement from the desired receiver. In this way, the transmitter also minimizes the energy consumption.

To save energy for future communications, the nodes using the TR-MAC protocol can form a pair by remembering each other's next wake-up time and a communicating frequency offset for communicating in future. Thus, the next time both the transmitter and the receiver node in the pair wake up at the previously agreed time instance and a particular frequency offset to communicate in the synchronized link state. In this way, the transmitter can optimally minimize the iteration of preamble-data and acknowledgement listen iteration to as small as possible; thereby can achieve energy-efficiency by minimizing the power penalty due to high transmission power of TR modulation. Moreover, in this final stage, the nodes can adapt their duty cycles to variable traffic while maintaining energy-efficiency to meet the requirements of the network, which is elaborated in Chapter 4. The TR-MAC protocol solves the critical multiple access issue using individual frequency offsets for individual pairs of nodes when the pair of nodes agrees on their future communicating time and frequency offset, a key advantage offered by the underlying TR modulation. Thus, collision can be avoided as future communication takes place in different virtual channels using different frequency offsets, hence, reduces the expense of costly retransmissions. This technique is elaborated in Chapter 5. Furthermore, the process of agreeing for a time instance and frequency offset for a pair of nodes for future communications is elaborated in Chapter 6. 


\subsubsection{Achieving energy-efficiency}

The main sources of energy waste, as discussed in Chapter 2, were i) idle listening, ii) control packet overhead, iii) collision, iv) overhearing. Idle listening refers to the situation when a node spends energy for a possible packet reception, but does not receive any packet. Allowing the sensor node to turn off its transceiver most of the time, and sample the channel for any activity for a very small amount of time saves energy. In this way, the sensor node is able to minimize the energy consumption due to idle listening. One node using TRMAC protocol can choose its wake up time instant by itself, and does not need to share that information with other nodes in the network. Therefore, TR-MAC protocol eliminates the need of extra information exchange for the networkwide synchronization of wake up times for the nodes in the WSN. As a result, TR-MAC protocol saves energy by minimizing the control packet overhead to communicate this extra synchronization information, and minimizes the complexity since nodes do not require maintaining a network-wide schedule of wake up times of all the nodes. Furthermore, collisions are also reduced, because of the less information exchange for maintaining the network-wide schedule in the WSN. In addition, the intended receiver and the overhearers can decode the destination node address from the preamble part of the preambledata transmitted by the transmitter. Hence, the potential overhearers can return to sleep after receiving one preamble-data packet, thus minimizing the energy waste due to overhearing. Finally, the synchronized link state in TR-MAC protocol minimizes communication in the medium by allowing the transmitter node to communicate precisely when the receiver node wakes up. As a result, the collision and overhearing in the communication medium is minimized.

\subsubsection{Protocol operation}

The proposed TR-MAC protocol has three operating states, namely (1) first time communication, (2) unsynchronized link state, and (3) synchronized link state; as shown in Figure 3.1. In the beginning of operation, a node knows nothing about the system and its neighbors. This state is referred to as the first 


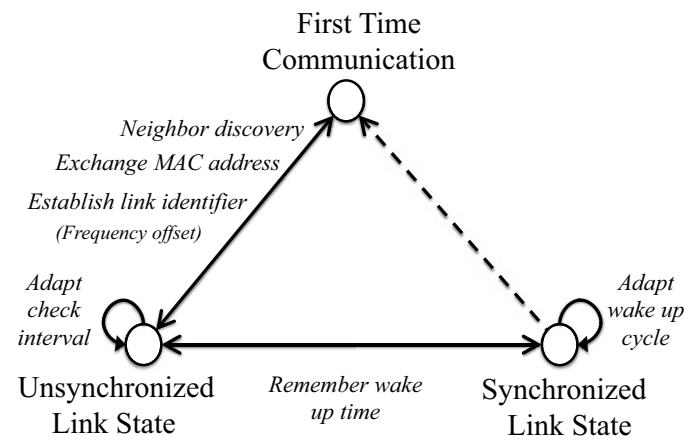

Figure 3.1: TR-MAC: Operating states

time communication state. The first time communication state is presented as a separate link state in Figure 3.1 to perform the initial neighbor discovery related tasks. In this state, one node performs neighbor discovery, exchanges MAC address, and establishes link identifiers. Once the node completes the three tasks mentioned above, the protocol can move to operate in unsynchronized link state. The protocol operation in first time communication state takes place in the same way as in unsynchronized link state. Thus, the protocol operation of the first time communication state is explained in the next paragraph, where the unsynchronized link state is explained. A number of frequency offsets are available using TR modulation, and one of the frequency offset is assigned as the default frequency offset for the common channel. All the initial communications takes place using this common channel with the default frequency offset, which is known globally in the network. The rest of the frequency offsets are used in the synchronized link state, where nodes form pair to communicate with each other using a particular previously agreed frequency offset. Figure 3.2 represents the TR-MAC protocol operation for unsynchronized and synchronized link states. The relevant system parameters with their corresponding symbols are presented in Table 3.1.

In unsynchronized link state, all the nodes in the system sleep most of the time, and perform channel sampling periodically in the default frequency offset for 


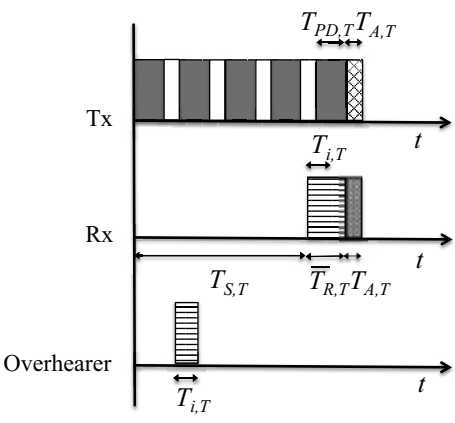

Unsynchronized Link State

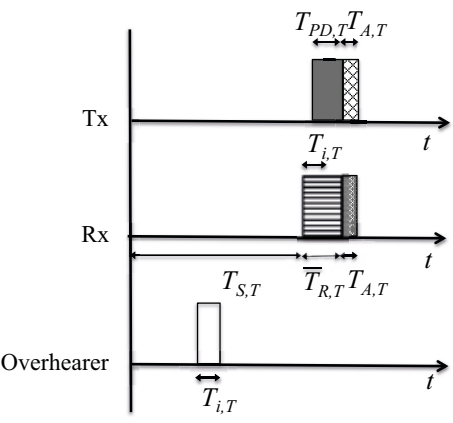

Synchronized Link State
Preamble
with data

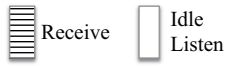

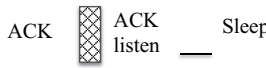

Figure 3.2: TR-MAC protocol operation

\begin{tabular}{|ll|}
\hline Symbols & System Parameters \\
\hline$T_{P, T}$ & Preamble duration \\
$T_{H, T}$ & Header duration \\
$T_{D a t a, T}$ & Data duration \\
$T_{P D, T}$ & Preamble + header + data duration \\
$T_{A, T}$ & Acknowledgement duration \\
$T_{i, T}$ & Periodic listen duration \\
$T_{S, T}$ & Sleeping duration \\
$T_{W, T}$ & Check interval duration \\
$\bar{T}_{R, T}$ & Expected receiving duration \\
$P_{T x, T}$ & Power to send \\
$P_{R x, T}$ & Power to receive \\
$P_{S, T}$ & Power to sleep \\
$\lambda$ & Packet arrival rate \\
$n$ & Number of sensor nodes \\
\hline
\end{tabular}

Table 3.1: System parameters

any data transmission. Each node can choose its periodic channel sampling time independently, and does not need to share this information to its neighbors. Therefore, for a successful communication, the transmitter transmits preamble- 
data using the default frequency offset, waits for the acknowledgement from the desired receiver. The transmitter repeats this procedure until the maximum duration of the check interval to ensure that all its neighbors will receive at least one preamble-data packet in the iteration. The check interval is the duration between the sleeping time of two successive periodic channel sampling times of a node, and is known to all nodes globally from the beginning. If the intended receiver receives a single preamble-data packet, then it responds with an acknowledgement. The transmitter stops these iterations after receiving an acknowledgement from the desired receiver. This complete process indicates a successful transmission of a data packet, as explained in the Figure 3.2 for the unsynchronized link state. The receiver can derive the destination address from the preamble part of the preamble-data packet. As a result, potential overhearers can return to sleep after decoding only the preamble part of the completely received preamble-data packet.

In order to move to synchronized state, the transmitter and receiver pair has to agree on future communication time instance, and a frequency offset from the frequency offset pool. Therefore, for a successful communication in the synchronized link state, the receiver node in the pair wakes up at a previously agreed time instance and frequency offset to check any channel activity, and the transmitter node in the node pair also transmits in the same time instance using the same frequency offset. In this way, the transmitter can optimally minimize the preamble-data and acknowledgement listen cycle duration to as small as possible for the future communications, and the receiver can minimize its idle listening duration; as shown in Figure 3.2 for the synchronized link state. Consequently, the nodes can communicate in an energy-efficient manner in this state, which is important for the TR-MAC protocol because of the inherent transmission power penalty of using TR modulation. The agreement about the future wake up time and frequency offset for a pair of nodes can happen in two ways: using a receiver-driven or a transmitter-driven strategy. For the receiver-driven case, the receiver allocates an extra wake up at a particular frequency offset, and the transmitter delays the next packet transmission until that receiver's next pairwise channel sampling time in that particular previously 
agreed frequency offset. This refers to the fact that the transmitter follows the receiver; hence the term receiver-driven communication. For the transmitterdriven case, the transmitter proposes a time instance, and the receiver adds an extra channel sampling time. Thus, the receiver follows the transmitter, hence the term transmitter-driven communication. The details of receiverdriven and transmitter-driven communication techniques, and the process of communicating the next wake up time are presented in Chapter 6 . In both of these mechanisms, both of the nodes still continues their respective periodic listening in the default frequency offset for communicating with other unpaired nodes in the unsynchronized link state.

The MAC layer enjoys tremendous flexibilities because of the options to communicate in either receiver-driven or transmitter-driven strategy for both link layer, and multi-hop communication. For example, one node having less energy can transfer energy burden by requesting another node to follow its lead, and can effectively operate like an energy-driven protocol. Efficient broadcasting can take place where one node makes its first-hop neighbors to follow its lead.

\subsection{Analytically modelling TR-MAC protocol}

In this section, we present a mathematical representation of the TR-MAC protocol for both the unsynchronized and the synchronized link state in terms of energy consumption. The analytical model computes the total energy consumption for a node to perform its necessary tasks; for example, to perform periodic listening, to receive a packet, and to send a packet. The system parameters used to model the energy consumption are summarized in Table 3.1 in Subsection 3.2.2. The protocol operation in the unsynchronized and synchronized link state is presented in Figure 3.2 in Subsection 3.2.2.

\subsubsection{Unsynchronized link state:}

TR-MAC is a preamble sampling protocol, thus this protocol allows a node to sleep most of the time. However, the node has to wake up periodically to sense 
the channel for any activity. If the desired node receives the beginning preamble part within its periodic listening, then it continues to listen for the remaining data before sending the acknowledgement back to the sender. However, if the node receives the end part of the preamble-data packet within its periodic listen duration, then it extends its periodic listening to listen for the next complete preamble-data packet. The overhearing nodes can go back to sleep by turning off their transceiver after they receive the preamble part of the preamble-data packet without sending any acknowledgement. This becomes possible since the preamble part contains the destination address.

The total energy for TR-MAC to operate in the unsynchronized link state is given by Equation 3.1:

$$
E_{T}^{u n s y n c}=\lambda\left(E_{T x, T}^{u n s y n c}+E_{R x, T}^{u n s y n c}+(n-2) E_{O H, T}\right)+n E_{P L, T}
$$

where $E_{T}^{\text {unsync }}$ represents the energy consumption of the total system in the unsynchronized link state of the TR-MAC protocol, $\lambda$ being the packet arrival rate, $E_{T x, T}^{\text {unsync }}$ is the energy to transmit a packet, $E_{R x, T}^{\text {unsync }}$ is the energy to receive a packet, $E_{O H, T}$ is the overhearing energy, and $E_{P L, T}$ is the energy for periodic listening. We assume $n$ number of nodes where one node transmits, another node listens, and $(n-2)$ other nodes act as overhearers.

Firstly, we calculate the periodic listening energy, $E_{P L, T}$, of the TR-MAC protocol. Every node using the TR-MAC protocol performs periodic listening for duration $T_{i, T}$, and sleeps for the duration $T_{S, T}$, within one cycle of check interval duration, $T_{W, T}$. Recall that the check interval, $T_{W, T}$, is the duration between two consecutive periodic listening duration of a node. Therefore, the energy spent for a single cycle of periodic listening and sleeping is given by $\left(P_{R x, T} T_{i, T}+P_{S, T} T_{S, T}\right)$, where $P_{R x, T}$ represents the power spent to receive for periodic listening duration $T_{i, T}$, and $P_{S, T}$ represents the power spent to sleep for sleeping duration $T_{S, T}$. Hence, the energy spent for the periodic listening, $E_{P L, T}$, is given by Equation 3.2:

$$
E_{P L, T}=P_{R x, T} T_{i, T}+P_{S, T} T_{S, T} .
$$


The goal of the periodic listening is to detect the data transmission in the medium. Therefore, the duration of the periodic listening needs to be long enough to detect any ongoing transmission, and at the same time, it needs to be short enough to minimize the power consumption. We consider that one node can detect an ongoing data transmission if it can listen at least the preamble duration of the preamble-data packet. Since the transmitter using the TR-MAC protocol waits for the acknowledgment between transmitting two consecutive preamble-data packets, thus the periodic listen duration, $T_{i, T}$, of a node has to be long enough to detect the preamble duration, $T_{P, T}$, of any of the two consecutive preamble-data packets including one acknowledgement packet duration, $T_{A, T}$, within these two consecutive preamble-data packets. If the periodic listening duration is shorter than the above duration, then one node may not be able to detect an ongoing transmission. Hence, the periodic listening duration, $T_{i, T}$, has to be greater than or equal to the duration of the acknowledgement duration, $T_{A, T}$, plus two times preamble duration, $T_{P, T}$, and is represented by Equation 3.3:

$$
T_{i, T} \geq T_{A, T}+2 T_{P, T}
$$

We take the minimum duration for the periodic listen of TR-MAC in our calculation to minimize the power consumption, because that is enough to detect a transmission.

The receiver using the TR-MAC protocol extends its periodic listening if it detects the preamble part of the preamble-data packet. Thus, the receiver has to spend extra energy to receive a packet in addition to the periodic listen energy. To calculate this additional energy for the reception of the data packet, the periodic listen duration, $T_{i, T}$, has to be subtracted from the expected extended listening duration, $\bar{T}_{R, T}$. Furthermore, the energy to send an acknowledgement has to be added. Hence, the additional energy to receive a packet, $E_{R x, T}^{\text {unsync }}$, is given by Equation 3.4:

$$
E_{R x, T}^{u n s y n c}=P_{R x, T}\left(\bar{T}_{R, T}-T_{i, T}\right)+P_{T x, T} T_{A, T} .
$$


Now we calculate the expected extended listening duration, and use that to calculate the energy spent for overhearing. The receiver's listen duration has to be at least the duration of a preamble-data packet in the best case scenario, considering the receiver wakes up exactly at the time to receive the preamble-data packet sent by the transmitter. However, consider a scenario where the receiver node wakes up during an ongoing preamble-data packet transmission. Then the receiver node can detect that a transmission is ongoing, but cannot decode the packet successfully since the complete preamble-data packet was not received. In this case, the receiver node has to extend its its periodic listen duration to receive the next complete preamble-data packet. Since the transmitter using TR-MAC protocol waits for an acknowledgement from the receiver in between transmitting two consecutive preamble-data packets, thus the extended duration has to include one acknowledgement duration. Therefore, the periodic listening can have a minimum duration same as a single preamble-data packet duration, and can have a maximum duration of the first incomplete preambledata packet duration, plus an acknowledgement duration, plus the second complete preamble-data packet duration. The maximum duration considers the full first preamble-data packet reception duration, because missing only a few bits of this first packet results in an incomplete packet reception. Thus, the expression for receiver listen duration is given by Equation 3.5:

$$
T_{A, T}+2 T_{P D, T}>T_{R, T} \geq T_{P D, T}
$$

The probability that the receiver can wake up at any time within the interval $\left[T_{P D, T}, T_{A, T}+2 T_{P D, T}\right]$ is equal. That is why, we assume the wake up time of the receiver is uniformly distributed over the interval $\left[T_{P D, T}, T_{A, T}+2 T_{P D, T}\right]$. As a result, the expected extended listening duration, $\bar{T}_{\mathrm{R}, \mathrm{T}}$, is given by Equation 3.6:

$$
\bar{T}_{\mathrm{R}, \mathrm{T}}=\int_{t=T_{P D, T}}^{T_{A, T}+2 T_{P D, T}} t P\left(T_{R, T}=t\right) d t=\frac{1}{2} T_{A, T}+\frac{3}{2} T_{P D, T} .
$$

The overhearer listens to the complete preamble-data packet, finds that it is 
not the desired receiver node. Thus, the overhearer node does not send an acknowledgement. However, the in order to decode the destination address, the overhearer has to receive a complete preamble-data packet. As a result, if the overhear wakes up during the first preamble-data packet, and receives an incomplete preamble-data packet, then it has to extend its periodic listening to receive the second preamble-data packet. Hence, the overhearing energy is actually the energy spent in receiving a packet including the possible extension of periodic listening, but without the energy to send an acknowledgement. Thus, the overhearing energy is derived from Equation 3.4, and is given by Equation 3.7:

$$
E_{O H, T}=P_{R x, T}\left(\bar{T}_{R, T}-T_{i, T}\right)
$$

Finally, the energy to send a single packet for TR-MAC, $E_{T x, T}^{\text {unsync }}$, is given by Equation 3.8, and is derived as follows. The energy required for a single cycle of sending a packet of preamble-data, followed by listening for an acknowledgement (regardless of its reception) is given by $\left(P_{T x, T} T_{P D, T}+P_{R x, T} T_{A, T}\right)$. Always at least one such cycle is needed for sending the data, hence the +1 . Extra cycles might be needed depending on when the receiver wakes up. If we assume that the first cycle of sending a packet starts at an arbitrary moment between the start of two consecutive listen periods with duration $\left(T_{i, T}+T_{S, T}\right)$, we can derive the expected number of extra cycles. If the packet starts within the first $\left(T_{i, T}-T_{P, T}\right)$ seconds of the listen period of the receiver, no extra iterations are needed. This happens because the receiver receives the complete preamble from which it can derive that it needs to stay awake for the rest of the packet. If the packet transmission starts later, with probability $\left(T_{S, T}+T_{P, T}\right) /\left(T_{i, T}+T_{S, T}\right)$, extra preambledata and listen iterations are transmitted until the next listen period of the

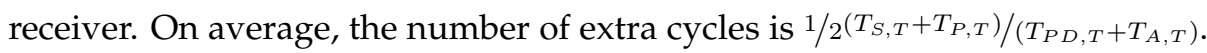


The energy needed to send a packet, $E_{T x, T}^{u n s y n c}$, is thus given by Equation 3.8:

$$
\begin{aligned}
E_{T x, T}^{u n s y n c} & =\left(\frac{1}{2} \frac{\left(T_{S, T}+T_{P, T}\right)^{2}}{\left(T_{i, T}+T_{S, T}\right)\left(T_{P D, T}+T_{A, T}\right)}+1\right) \\
& \times\left(P_{T x, T} T_{P D, T}+P_{R x, T} T_{A, T}\right) .
\end{aligned}
$$

\subsubsection{Synchronized link state:}

In the synchronized link state of the TR-MAC protocol, a transmitter and receiver pair wake up at a previously agreed upon time to communicate at a particular frequency offset. Even though the two communicating nodes try to wake up at a particular previously agreed upon time, yet there can be timing mismatch in waking up with respect to each other. This happens because of the respective clocks in those two nodes being drifted from the ideal depending on the time since the last communication. The total energy of the system in the synchronized link state is calculated for a particular link without any overhearers, since nodes are now synchronized and follow each other's wake up time. All the nodes have to perform their respective periodic listening in the default frequency offset to communicate with the unpaired nodes. Hence, the total energy per second in synchronized link state is given by

$$
\left.\left.E_{T}^{s y n c}=\lambda\left(\bar{E}_{T x, T}^{s y n c}\right]+\bar{E}_{R x, T}^{s y n c}\right]\right)+n E_{P L},
$$

where $E_{T x, T}^{s y n c}$ represents the expected energy to transmit a packet given by Equation 3.10, and $E_{R x, T}^{s y n c}$ represents the expected energy to receive a packet given by Equation 3.11 respectively

$$
\begin{aligned}
& \bar{E}_{T x, T}^{s y n c}=\int_{d=d_{\min }}^{d=d_{\max }} P(D=d) E_{T x, T}^{s y n c} \mid(D=d) \mathrm{dd}, \\
& \bar{E}_{R x, T}^{\text {sync }}=\int_{d=d_{\text {min }}}^{d=d_{\max }} P(D=d) E_{R x, T}^{s y n c} \mid(D=d) \mathrm{dd} .
\end{aligned}
$$


The probability part of the previous two equations is derived from the possible clock difference between the transmitter and receiver's clock, represented by the random variable $D$ with individual realization $d$. The energy part calculates the amount of energy needed to transmit or receive a packet for individual clock differences. The equations to calculate the energy are provided later.

The main reason for the difference between the clocks of transmitter and receiver node is the clock drift. We assume that the clock drift of both transmitter and receiver nodes, represented by $C_{T}$ and $C_{R}$ respectively, are uniformly distributed, as in [62]. Therefore, we have

$$
P\left(C_{T}=c\right)=P\left(C_{R}=c\right)=\left\{\begin{array}{cc}
\frac{1}{2 \theta l}, & -\theta l \leq c \leq \theta l \\
0, & \text { otherwise }
\end{array}\right.
$$

where $\theta$ represents the frequency tolerance of the clock, $l$ represents the time since last communication, and $c$ represents the individual realizations of $C_{T}$ and $C_{R}$. For example, the receiver might be $\theta l$ seconds early, or $\theta l$ seconds late compared to the transmitter based on the clock drift, hence the term $2 \theta l$. In contrast, the transmitter can also be $\theta l$ seconds early, or $\theta l$ seconds late with respect to the receiver. We are interested in the difference of the two clocks $C_{T}-C_{R}$, that is, how much the sender and receiver clocks are apart from each other. The difference between two uniformly distributed clock drifts results in a convolution between them that further produces a triangular distribution, which eventually determines the probability of the transmitter and receiver being awake to communicate. This probability is given by

$$
P(D=d)=\left\{\begin{array}{cc}
\frac{2 \theta l+d}{(2 \theta l)^{2}}, & -2 \theta l \leq d \leq 0, \\
\frac{2 \theta l-d}{(2 \theta l)^{2}}, & 0<d \leq 2 \theta l, \\
0, & \text { otherwise. }
\end{array}\right.
$$

In the synchronized link state, the transmitter has to transmit at least one preamble-data packet, and may be more dependent on the precise wake-up time of the receiver. Receiving an acknowledgement from the receiver marks a 


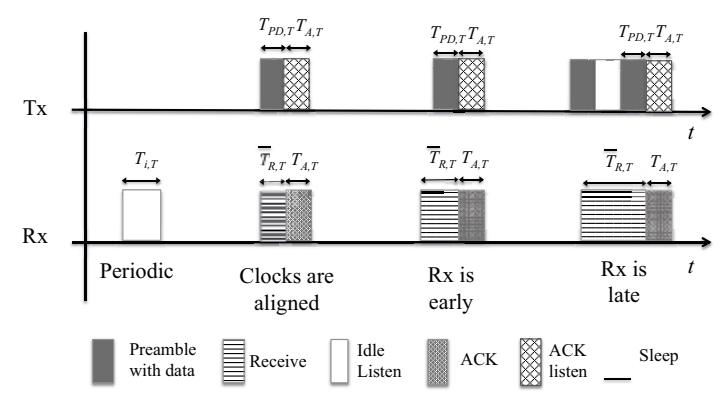

Figure 3.3: TR-MAC synchronized link state

successful communication. The energy to transmit a packet in the synchronized link state can be calculated by

$$
E_{T x, T}^{s y n c}=P_{T x, T} T_{P D, T}+P_{R x, T} T_{A, T}+N \times\left(P_{T x, T} T_{P D, T}+P_{R x, T} T_{A, T}\right),
$$

where $N$ represents the number of extra preamble-data packets. Recall that, the transmitter node waits for the acknowledgement after transmitting every preamble-data packet. Figure 3.3 represents different scenarios that might take place in the synchronized link state. The transmitter does not need to transmit any extra preamble-data packets if it is later than the receiver, meaning that the receiver node can receive the complete preamble-data packet. Hence, $N$ becomes zero for this case. However, if the transmitter is early, then it has to transmit more than one preamble-data packets based on the positive value of $N$, thus it spends much energy in this scenario. The positive fractional value of $N$ is rounded to the next integer value, since the transmitter has to transmit at least one more preamble-data packet. Here $N$ is calculated depending on the specific clock drift, and is given by

$$
N=\left\lceil\frac{\max (d, 0)}{T_{P D, T}+T_{A, T}}\right\rceil .
$$


The receiver in the synchronized link state spends energy to receive the data, later transmits an acknowledgement after successful reception. The receiver has to listen more than the ideal duration, if it wakes up either early or late with respect to the transmitter. If the receiver is early, then it has to continue listening until it receives the complete preamble-data packet. Alternatively, if the receiver is late, then it receives part of the first preamble-data packet, and cannot decode that packet. Therefore, if the receiver node receives at least the preamble duration of the first preamble-data packet, then it has to continue listening to receive one more preamble-data packet transmitted by the transmitter. After receiving a complete preamble-data packet, the receiver node transmits an acknowledgement back to the sender node. These alternative situations that can take place in the synchronized link state are explained in the Figure 3.3. Therefore, the receiver in the unsynchronized link state spends energy for receiving the one complete preamble-data packet, sending one acknowledgement packet, and additional receiving energy for receiving a partial preamble-data packet depending on the wake up time of the receiver relative to the transmitter. Hence, the energy spent by the receiver is given by

$$
E_{R x, T}^{s y n c}=P_{R x, T} T_{P D, T}+P_{T x, T} T_{A, T}+P_{R x, T} \times\left(N\left(T_{P D, T}+T_{A, T}\right)-d\right) .
$$

\subsection{TR-MAC protocol optimization}

TR-MAC protocol has been analytically modelled and presented in Section 3.3. However, optimizations have been proposed on the basis of the analytical modelling of the TR-MAC protocol both in the unsynchronized and the synchronized link state. The optimizations of the TR-MAC protocol, which have been first proposed in $[20,21]$, are presented in this section.

\subsubsection{Unsynchronized link state}

In the unsynchronized link state of the TR-MAC protocol, each node can choose its periodic channel sampling time independent of other nodes, and does not 
need to share this information to its neighbor nodes. In this way, TR-MAC protocol follows the basic principle of preamble sampling protocol. Since the transmitter node does not know when the potential receiver will wake up, thus it has to take the responsibility of reaching the receiver during its periodic listening time. Therefore, for a successful communication, the transmitter sends preambledata in the default frequency offset, then waits for the acknowledgement from the desired receiver, and repeats this procedure until the maximum check interval duration to ensure that all nodes receive at least one preamble-data during its periodic listening time. That is why, the maximum check interval duration has to be globally known in the network. Once the desired receiver receives a preamble-data packet during its periodic listening, then it responds with an acknowledgement. Then the transmitter stops the iteration of preambledata packets, and does not continue till the maximum check interval duration.

From the above operation in the unsynchronized link state, we can observe that the overall energy consumption depends on the chosen check interval duration, since it has an impact on both the receiver and the transmitter. Choosing a very small check interval makes all the receiver nodes to wake up frequently, thus the receiver nodes waste energy. However, choosing a very small check interval has a better consequence for the energy consumption of the transmitters, because the maximum duration for the repetition of preamble-data, which depends on the chosen check interval, becomes smaller. In contrary, choosing a very long check interval saves energy on the receiver nodes, because they can sleep for a longer duration by having infrequent periodic listening. But it means that the transmitter nodes have to continue the repetition for preamble-data packet for a longer duration, thus spending a significant amount of energy. Therefore, the check interval duration can be optimized to minimize the overall energy consumption in both the transmitter and the receiver side. One possible way of optimizing the check interval is to choose it depending on the expected packet arrival rate in the network. The application scenario determines the expected packet arrival rate in the network. Thus, the check interval can be optimized depending on the expected arrival rate of the network for the particular application scenario during the time of the deployment of the sensor network. In this 
way, all the nodes in the network can start with the optimized check interval duration from the beginning.

Therefore, we derive a mathematical expression to find the optimum check interval duration for the TR-MAC protocol depending on the packet arrival rate. The transmitter side spends a significant amount of energy for the repetition of the preamble-data packet for a maximum of the check interval duration compared to the receiver side. Thus, we take the energy consumption of the transmitter side into consideration for optimizing the check interval duration. We find out this optimum by first differentiating the equation for energy consumption in the transmitter side with respect to the check interval duration, then evaluate the differentiated result for the check interval duration. Thus, we take the mathematical expressions of Equation 3.1, and differentiate with respect to the check interval, $T_{W, T}$, which is given by Equation 3.17:

$$
\begin{gathered}
\frac{\mathrm{d} E_{T x, T}^{\text {unsync }}}{\mathrm{d} T_{W, T}}= \\
\frac{n P_{S, T}}{T_{W, T}}-\frac{n\left(P_{R x, T} T_{i, T}-P_{S, T}\left(T_{i, T}-T_{W, T}\right)\right)}{T_{W, T}^{2}} \\
-\left(\frac{\left(T_{P, T}-T_{i, T}+T_{W, T}\right)^{2}}{2 T_{W, T}^{2}\left(T_{A, T}+T_{P D, T}\right)}-\frac{2\left(T_{P, T}-T_{i, T}+T_{W, T}\right)}{2 T_{W, T}\left(T_{A, T}+T_{P D, T}\right)}\right) \\
\times \lambda\left(P_{R x, T} T_{A, T}+P_{T x, T} T_{P D, T}\right) .
\end{gathered}
$$

Afterwards, we find out that the optimized check interval, $T_{W, T}^{*}$, by evaluating Equation 3.17, and the result is given by Equation 3.18:

$$
\begin{gathered}
T_{W, T}^{*}=\left[\lambda ( P _ { R x , T } T _ { A , T } + P _ { T x , T } T _ { P D , T } ) * \left\{\lambda\left(P_{R x, T} T_{A, T}+P_{T x, T} T_{P D, T}\right)\right.\right. \\
\times\left(T_{i, T}^{2}+T_{P, T}^{2}\right)+2 n\left(T_{A, T} T_{i, T}+T_{P D, T} T_{i, T}\right)\left(P_{R x, T}-P_{S, T}\right) \\
\left.\left.-2 \lambda T_{P, T} T_{i, T}\left(P_{R x, T} T_{A, T}+P_{T x, T} T_{P D, T}\right)\right\}\right]^{1 / 2} \\
/ \lambda\left(P_{R x, T} T_{A, T}+P_{T x, T} T_{P D, T}\right) .
\end{gathered}
$$

This expression can be evaluated by supplying individual input values for respective variables to optimize the check interval. Here the expected packet 
arrival rate, $\lambda$, and the number of neighbor nodes, $n$, comes from the deployed application scenario for the sensor network, and has to be set during the time of the deployment.

\subsubsection{Synchronized link state}

In the synchronized link state of the TR-MAC protocol, the receiver node in the pair wakes up at a previously agreed time instance and a particular frequency offset to check any channel activity, and the transmitter node in the node pair also transmits in the same time instance using the same frequency offset. In this way, the transmitter can optimally minimize the preamble-data and acknowledgement listen cycle duration to as small as possible for the future communications, and the receiver can minimize its idle listening duration. The TR-MAC protocol in synchronized link state effectively minimizes its preambledata iterations in transmitter side, thus achieves energy-efficiency. The energy consumption in the synchronized link state depends on the relative wake up time based on the transmitter and the receiver clocks. If the clocks are aligned, then both the transmitter and the receiver spend minimum energy. The transmitter does not need to transmit any extra preamble-data packets, if the receiver wakes up earlier than the transmitter or at the same time. However, if the receiver wakes up later than the transmitter, then the transmitter needs to send more than one preamble-data packets. At the same time, the receiver needs to extend its receiving. This results in more energy consumption for both the transmitter and the receiver. This phenomenon of wake up time difference between the transmitter and the receiver is explained in Section 3.3 Figure 3.3. The fact that at system level, the receiver being late is costlier than the receiver being early, led us to experiment with an intentional clock offset, $\delta$. As a result, the expected energy to transmit or receive in Section 3.3 Equation 3.10, and 3.11 change their form. However, this clock offset only affects the probability part of the triangular distribution in Equation 3.13, not the energy part. The triangular distribution with a negative intentional clock offset of $\delta$ can be derived from 
Equation 3.13, and can be represented by

$$
P(D=d)=\left\{\begin{array}{cc}
\frac{2 \theta l+(d+\delta)}{(2 \theta l)^{2}}, & (-2 \theta l-\delta) \leq d \leq(0-\delta), \\
\frac{2 \theta l-(d+\delta)}{(2 \theta l)^{2}}, & (0-\delta)<d \leq(2 \theta l-\delta), \\
0, & \text { otherwise }
\end{array}\right.
$$

where $\theta$ represents the frequency tolerance of the clock, and $l$ represents the time since last communication. For a given clock drift of $\theta$, the intentional clock offset, $\delta$, can be calculated numerically depending on the last communication time.

\subsection{Comparison with reference protocols}

In this section, we present the analytical model for two reference protocols in the preamble sampling category, and compare our TR-MAC preamble sampling protocol with these reference protocols. The preamble sampling protocol with all its subcategories were presented in Chapter 2. We choose X-MAC [39] as the reference protocol for the preamble sampling protocols with preamble packetization. This is an ideal reference protocol from this category, since the destination address is mentioned in the preamble, and the transmitter waits from the acknowledgement from the receiver after sending each preamble packet. We choose WiseMAC [40, 63, 64, 65] for the schedule learning category of preamble sampling protocols, since a transmitter using WiseMAC remembers the next wake up schedule of the receiver to minimize the preamble for the future communications. Thus, we choose these X-MAC and WiseMAC protocol as the reference protocols, since the characteristics of these two protocols are comparable with the TR-MAC preamble sampling protocol.

The operating principles of X-MAC [39] and WiseMAC [40] protocols are presented in Figure 3.4 with their behavior in corresponding states together with TR-MAC protocol to facilitate the comparison. Here we see that X-MAC protocol only has the unsynchronized link state. This protocol does not have the synchronized link state, since it does not adapt its preamble-listen duration for 

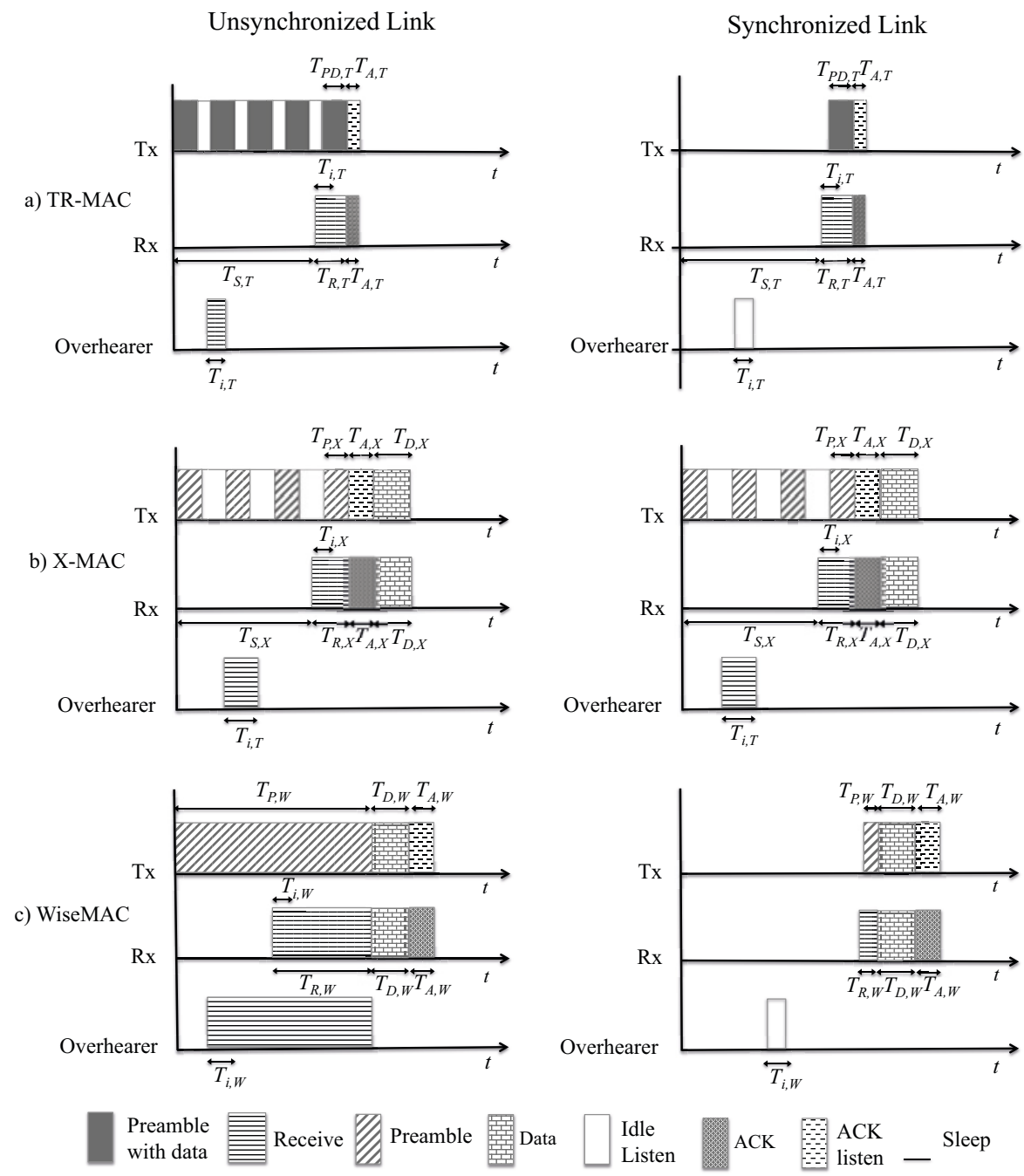

Figure 3.4: TR-MAC with two reference protocols

the future communications. The transmitter using the X-MAC protocol sends packetized preambles and waits for the acknowledgement from the receiver, 
and continues this cycle for a maximum of the check interval of the receiver, which is the duration of two consecutive wake up times of the receiver. X-MAC has high extra packet overhead, because this same cycle has to be repeated to send each packet from a source to a destination node. Also X-MAC does not send any acknowledgement after successful data packet transmission, it only acknowledges the reception of the preamble packet.

Alternatively, in Figure 3.4, we see that WiseMAC protocol has both the unsynchronized and the synchronized link state. In the unsynchronized link state, WiseMAC has to use a long preamble before transmitting the data packet. As a result, the potential receiver and the overhearers have to listen the complete preamble for the first time communication before receiving any data, which is costly for per packet overhead in low traffic condition. Furthermore, WiseMAC has to use long preambles for broadcasting instead of short ones, and does not adapt duty cycles depending on the changing traffic pattern. To move to the synchronized link state, the receiver node includes the next wake up time in the acknowledgement. Thus, the transmitter adapts the preamble length in the later state by remembering the receiver's next periodic wake up time. In synchronized link state, WiseMAC efficiently manages the effects of clock drifts and minimizes collision using small preambles. The receiver-driven approach of WiseMAC makes a potential receiver collision-prone if many transmitters follow one node's next wake up and start transmitting at the same time. To have a remedy to this problem, WiseMAC adds a medium reservation preamble in front of the wake up preamble [40].

\subsubsection{Unsynchronized link state}

In this section, we present the model for the unsynchronized link state for the $X$ MAC and WiseMAC protocol. The energy consumption in the unsynchronized link state includes three activities: energy for periodic listening, energy to receive a packet, and energy to transit a packet, similar to that for TR-MAC protocol given by Equation 3.2, 3.4, and 3.8.

The energy spent for periodic listening for X-MAC protocol remains similar as TR-MAC protocol given in Equation 3.2. The condition for periodic listening 
for X-MAC, $T_{i, X}$, is given by Equation 3.20:

$$
T_{i, X} \geq T_{A, X}+2 T_{P, X}
$$

We take the minimum value of $T_{i, X}$ for calculation of the periodic listening duration for X-MAC protocol.

The additional energy spent to receive a packet for X-MAC does not include the energy to receive a preamble, because that is already calculated within the periodic listen energy. Therefore, only the energy to send an acknowledgement, and the energy to receive the data packet is present in the expression to calculate the additional energy to receive a packet, $E_{R x, X}^{u n s y n c}$. This additional energy to receive a packet is given by

$$
E_{R x, X}^{u n s y n c}=P_{T x, X} T_{A, X}+P_{R x, X} T_{D, X}
$$

Similarly, the energy to send a packet for X-MAC, $E_{T x, X}^{\text {unsync }}$, is derived like TR$\mathrm{MAC}$, and given by Equation 3.22 with the exception that X-MAC needs to send the data packet separately.

$$
\begin{aligned}
E_{T x, X}^{\text {unsync }} & =\left(\frac{1}{2} \cdot \frac{\left(T_{S, X}+T_{P, X}\right)^{2}}{\left(T_{i, X}+T_{S, X}\right)\left(T_{P, X}+T_{A, X}\right)}+1\right)\left(P_{T x, X} T_{P, X}+P_{R x, X} T_{A, X}\right) \\
& +P_{T x, X} T_{D, X}
\end{aligned}
$$

For WiseMAC, the minimum listen duration, $T_{i, W}$, is taken as the minimum preamble duration, $T_{P, T}$, because the receiver keeps listening in case it detects any transmission in the channel. The receiver using WiseMAC protocol has to spend additional energy to listen for the preamble, then to listen for the data packet, at last to send the acknowledgement. Hence, the energy to receive a packet for WiseMAC protocol, $E_{R x, W}^{u n s y n c}$, is given by 


$$
E_{R x, W}^{u n s y n c}=P_{R x, W}\left(\bar{T}_{R, W}-T_{i, W}\right)+P_{R x, W} T_{D, W}+P_{T x, W} T_{A, W},
$$

where the average receiver listen duration is $\bar{T}_{R, W}=\frac{T_{W}}{2}$. The energy to send a packet for WiseMAC includes the energy to send a preamble, then to send the data, and later to receive the acknowledgement. Thus, the energy to send a packet in WiseMAC protocol, $E_{T x, W}^{u n s y n c}$, is given by

$$
E_{T x, W}^{u n s y n c}=P_{T x, W} T_{P, W}+P_{T x, W} T_{D, W}+P_{R x, W} T_{A, W} .
$$

\subsubsection{Synchronized link state}

In this section, we model the synchronized link state of the WiseMAC protocol only, since the X-MAC protocol does not have any synchronized link state. The WiseMAC protocol for the synchronized link state has been modelled by considering similar equations mentioned for the TR-MAC protocol in Equation 3.10 and 3.11. Here the probability part of the equation and the resulting triangular distribution mentioned in Equation 3.13 remains the same, only the energy part differs. Thus, we model the energy part of WiseMAC.

Since the WiseMAC transmitter knows the receiver's next wake up time, it can transmit a preamble of duration $T_{P, W}=\min \left(4 \theta l, T_{W}\right)$, where $T_{W}$ is the periodic check interval. This value of the preamble is enough for the possible clock drifts of the transmitter and receiver, and the maximum value of $T_{W}$ ensures that the next periodic channel sampling receives the preamble. Hence, the energy for WiseMAC transmitter is given by

$$
E_{T x, W}^{s y n c}=P_{T x, W} T_{P, W}+P_{T x, W} T_{D, W}+P_{R x, W} T_{A, W},
$$

where $P_{T x, W}$ represents the power to transmit a packet, $P_{R x, W}$ represents the power to receive a packet, $T_{D, W}$ represents the data packet duration, and $T_{A, W}$ represents the acknowledgement duration. 
The WiseMAC receiver spends energy to detect the preamble, to receive the data packet, and to transmit the acknowledgement back to the transmitter. The energy for WiseMAC receiver is given by

$$
E_{R x, W}^{s y n c}=P_{R x, W} T_{P, W}+P_{R x, W} T_{D, W}+P_{T x, W} T_{A, W},
$$

where preamble receiving duration, $T_{P, W}$, is considered to have the same value as the minimum duration to detect communication of TR-MAC protocol, $T_{P, T}$.

\subsection{Validation of analytical modelling}

To validate the analytical modelling for TR-MAC protocol, we implemented the protocol in a simulator. The validation is achieved by comparing the results received from the simulator with the results received from the models.

\subsubsection{TR-MAC implementation in the simulator}

Simulation is an important technique to evaluate the mathematical model, since the system can be realized and tested in a controllable manner in a simulator. Thus, we implement TR-MAC protocol in OMNeT++ simulator with MiXiM simulation framework [66]. OMNeT++ is a discrete event simulator that helps to analyze wired and wireless protocols by means of events occurring at discrete time instances. An event can be a certain action, for example, generating a packet following a certain distribution, or transmitting a packet at a certain time. The simulated protocol causes the node to change its states between sleeping, transmitting, or receiving at discrete point of time depending on the events. All these changes can be analyzed based on the progression of time together with the relative ordering of the events.

MiXiM is a simulation framework that uses the OMNeT++ simulator for simulating wireless communication scenarios [66]. MiXiM provides some example protocol stacks for all the layers of OSI model with battery elements for computing energy consumption. Since we are interested to see the TR-MAC protocol behavior, we remodeled the MAC layer according to the TR-MAC protocol, 
and used the default implementation for the rest of the layers. The individual battery parameters are configured in MiXiM together with the power levels. The default network layer is used, and the sensor application layer is configured with Poisson packet arrival with exponential inter-arrival times between packets. The physical layer uses simple path loss models without any specific channel effects or propagation delays.

We present a finite state machine for the TR-MAC protocol implementation in Figure 3.5 with the possible states and events causing the node to change between its working states of sleeping, transmitting, and receiving. Each and every node has an instance of this state machine to perform its necessary tasks. If one node is communicating in the unsynchronized link state, then it does not need to keep track of any information for its neighbor nodes. However, each node needs to maintain a data structure for each of its neighbors after making a pair with that neighbor node, and moving from the unsynchronized link state to the synchronized link state. In the synchronized link state, the current node maintains the future communicating frequency offset and the time instance for its pairwise neighbor node to communicate in future. The data structure is organized in such a way that it keeps track of the next event, which can be a periodic listening in the default frequency offset, or a synchronized wake up or packet sending time for a paired node at a particular frequency offset. The current node schedules the next event, and then goes to sleep. When it wakes up again, then it checks the data structure to check the event to perform now, then performs that event. Before adding a new entry for a pairwise node in the data structure, the current node has to check for time overlapping event in the data structure. The node adds a new entry only if there is no time overlapping event in the data structure. The periodic listening activity in the default frequency offset for each node is the highest prioritized event in order to facilitate the information receiving from other nodes, which is the basic requirement for a preamble sampling protocol. The process of agreeing for a particular frequency offset and a time instance to move from the unsynchronized link state to the synchronized link state, and to fall back from the synchronized link state to the unsynchronized link state is further explained in Chapter 6. 


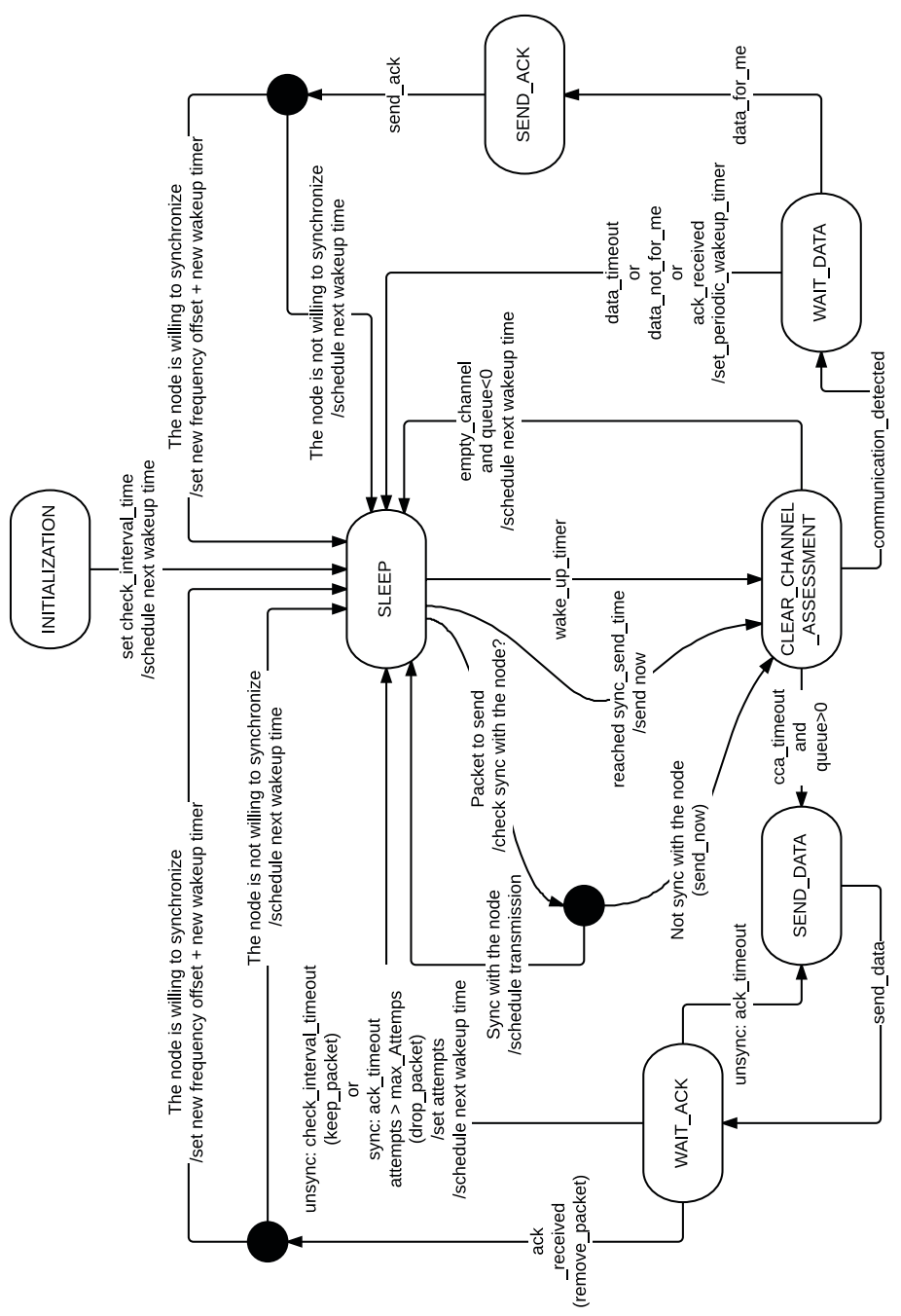

Figure 3.5: Finite state machine of TR-MAC protocol implementation

After initialization in INITIALIZATION state for the first time, the node starts in the unsynchronized link state and goes to SLEEP. One node wakes up to sense the channel for periodic listening duration by moving from the SLEEP 
state to the CLEAR_CHANNEL_ASSESSMENT state. If this node does not receive anything during CLEAR_CHANNEL_ASSESSMENT, and does not have any packet to send, then it goes back to SLEEP after scheduling its next periodic listening event after the check interval duration. If the node detects communication during CLEAR_CHANNEL_ASSESSMENT, then it goes to WAIT_DATA state. The current node remains in the WAIT_DATA state until it receives a complete preamble-data packet. If it detects a partial preambledata packet during the CLEAR_CHANNEL_ASSESSMENT state, then it has to remain in the WAIT_DATA state to receive at least one complete preamble-data packet. If the received preamble-data packet is meant for this node, and the received packet is a not an acknowledgement packet; then this current node needs to send an acknowledgement back to the sender node from whom it received the preamble-data packet. Before sending the acknowledgement, this current node also decides whether to move to the synchronized link state for receiver-driven communication, or to remain in the unsynchronized link state for this node. To move to the synchronized link state with this neighbor, this node allocates a non-overlapping time instance and a frequency offset for that neighbor node, if there exists no entry in the data structure for that neighbor. Afterwards, this current node sends the acknowledgement back to the sender neighbor node, schedules the next wake up event, and goes to SLEEP. Next time this node wakes up to perform its periodic listening, or for an agreed time instance to receive or send a packet for a pairwise node in the synchronized link state; depending on which comes first.

When one node receives a packet from the upper layer to send to a particular node, then it checks in the data structure whether there is already an entry for that particular node. If there exists an entry for that neighbor node, then this node realizes that it already has an agreement with that node for the future communication time instance and frequency offset. Therefore, it delays the packet transmission to that time instance, and goes back to SLEEP after scheduling the packet sending event to that particular paired node. If there exists no entry in the data structure for that particular node, then this current node realizes that it has to communicate in the unsynchronized link state with that particular node. 
Hence, this current node moves to the CLEAR_CHANNEL_ASSESSMENT state from the SLEEP state, and further moves on to SEND_DATA state to send the packet right away if the channel is empty. After sending the preamble-data packet in the SEND_DATA state, the node moves to WAIT_ACK to wait for an acknowledgement from the receiver node. The node iterates in these two states of SEND_DATA and WAIT_ACK until it receives an acknowledgement or until the default check interval time is reached. After receiving the acknowledgement packet with all the information from the receiver node in the receiver-driven communication, this node decides about going to synchronized link state by checking the data structure about the non-overlapping time instance. This current node adds the information regarding the neighbor node in the data structure if no overlapping time instance is present in the data structure. The node drops the packet after a maximum number of failing attempts, when it is unable to receive an acknowledgement for several check interval durations for the intended receiver node.

\subsubsection{Results and analysis}

In this section, we evaluate the analytical models together with the simulation results for TR-MAC, X-MAC, and WiseMAC protocol from the context of energy consumption. We simulate TR-MAC protocol using MiXiM framework in $\mathrm{OMNeT}++$. For generating simulation results, we run each simulation to generate and transmit approximately 100 packets, and the consumed energy is averaged for the number of packets. Afterwards, 100 of such simulation runs are used to average the result. We present the energy consumption results with respect to the following parameters, namely the check interval, and the packet arrival rate. We consider Poisson packet arrival with exponential inter-arrival times between packets.

The TR-MAC data packet, $T_{P D}, T$, consists of 8 bits of preamble, $T_{P, T}, 16$ bits of header, $T_{H, T}, 32$ bits of data, $T_{D a t a, T}$; thus having 56 bits. We consider the minimum duration for detecting any transmission for TR-MAC protocol corresponds to the preamble duration of 8 bits. Data rate of $25 \mathrm{kbps}$ is chosen for measurements. The duration of the preamble-data packet in the simulator is 


\begin{tabular}{|c|c|c|c|c|}
\hline Symbol & Description & TR-MAC & X-MAC & WiseMAC \\
\hline$T_{P}$ & Preamble duration & 8 bits & 65 bits & $T_{\mathrm{W}}$ \\
\hline$T_{H}$ & Header duration & $16 \mathrm{bits}$ & $16 \mathrm{bits}$ & $16 \mathrm{bits}$ \\
\hline$T_{D a t a}$ & Data duration & 32 bits & 32 bits & 32 bits \\
\hline$T_{A}$ & Acknowledgement duration & 24 bits & 65 bits & 80 bits \\
\hline$T_{i}$ & Periodic listen duration & $40 \mathrm{bits}$ & $195 \mathrm{bits}$ & $8 \mathrm{bits}$ \\
\hline$P_{T x}$ & Tx power & $2 \mathrm{~mW}$ & $1 \mathrm{~mW}$ & $1 \mathrm{~mW}$ \\
\hline$P_{R x}$ & Rx power & $1 \mathrm{~mW}$ & $1 \mathrm{~mW}$ & $1 \mathrm{~mW}$ \\
\hline$P_{S}$ & Sleep power & $15 \mu W$ & $15 \mu \mathrm{W}$ & $15 \mu \mathrm{W}$ \\
\hline
\end{tabular}

Table 3.2: System parameters values

calculated using the number of bits divided by the data rate. The periodic listen duration, $T_{i, T}$, is calculated using Equation 3.3 to be the acknowledgement duration, $T_{i, T}$, plus the twice of the preamble duration, $T_{P, T}$. The transmit power, receive power and sleep power are represented by $P_{T x, T}, P_{R x, T}$ and $P_{S, T}$ respectively. Since TR modulation has a power penalty due to sending the reference signal together with the modulation signal, thus it is considered that the transmitter using TR modulation spends twice power than any other protocols. The sleeping time duration, $T_{S, T}$, is calculated by subtracting the periodic listening duration, $T_{i, T}$, from the check interval, $T_{W, T}$. The switching time and power consumption to switch the transceiver among sleeping, sending and receiving states is considered as $15 \mu \mathrm{W}$ in the simulator. Since this value is much smaller compared to other power consumption values; thus it is neglected in the modeling. The preamble duration and the acknowledgement duration for the X-MAC protocol is taken from the protocol specification, and its periodic listening is calculated using the Equation 3.20 to be 195 bits. The acknowledgement duration for WiseMAC protocol is taken from its protocol specification. The periodic listening of WiseMAC protocol is taken as the minimum duration to detect any transmission, which is the preamble duration of 8 bits for the TR-MAC protocol. The header duration and the data packet duration for all the protocols are considered as same. The parameters and their corresponding representing symbols and values for TR-MAC, X-MAC, and WiseMAC protocols are given in Table 3.2. 


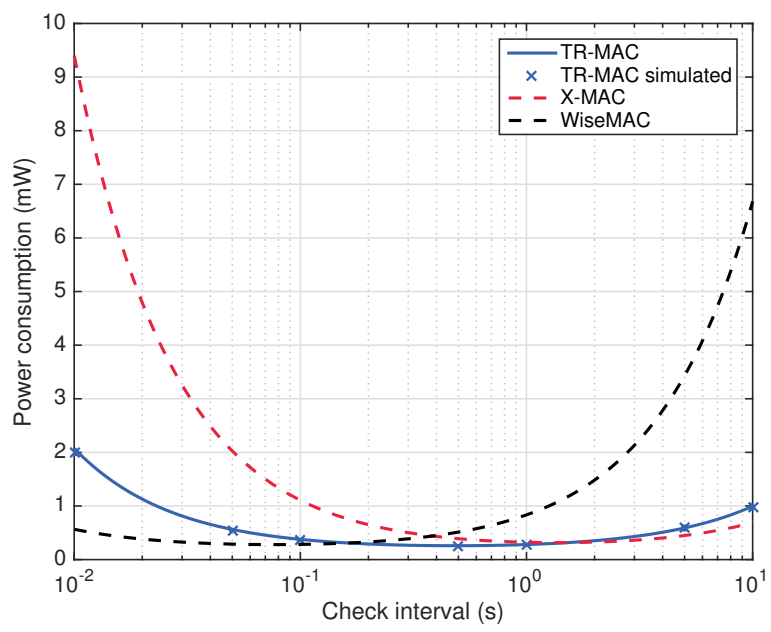

Figure 3.6: Unsynchronized link state: Power consumption for packet arrival rate $=0.1$ packets/second

\subsubsection{Unsynchronized link state}

The total energy consumption for the unsynchronized link state includes the energy to transmit or receive a packet, for periodic listening, and for overhearing. Here we consider a 1-hop link with a network of twelve nodes where one node is transmitting, another node is receiving, and ten other nodes are overhearing. We calculate and present the energy consumption in unsynchronized link state for all three protocols with respect to varying check interval for a traffic load of $\lambda=0.1$ packets/second in Figure 3.6. We present and explain the energy consumption results for a particular packet arrival rate, since similar system behavior for a given packet arrival rate has been examined. The TR-MAC simulation results, presented on top of the analytical results matches to the analytical ones. Confidence intervals are not drawn with the simulation results, because $95 \%$ of the values were smaller than $1 \%$ of the displayed value. We see that the power consumption for TR-MAC protocol has the minimum value in the middle of the check interval, and the power consumption increases for 


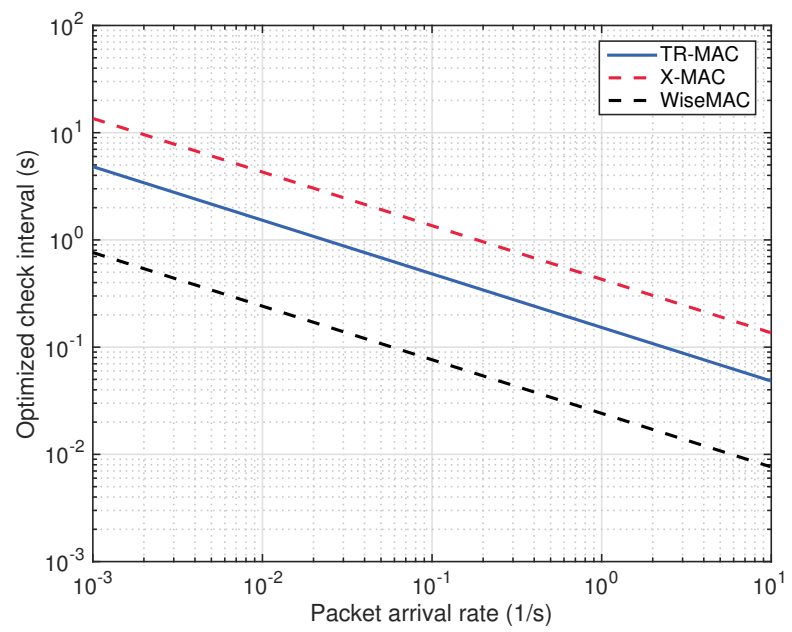

Figure 3.7: Unsynchronized link state: Optimized check interval for varying packet arrival rate

smaller or longer check interval values. For smaller check interval values, the periodic listening dominates the total power consumption. In contrary to that, the packet transmission dominates the total power consumption for longer check interval. The results also show that the energy consumption for TR-MAC is lower than X-MAC, but higher than WiseMAC for smaller check interval values. However, the scenario turns around when the check interval is longer. Therefore, the energy consumption for the TR-MAC protocol can be minimized by optimizing the check interval for a given traffic load.

In Section 3.4, we presented a mathematical expression to find the optimum check interval to minimize the energy consumption in the unsynchronized link state for TR-MAC protocol for a given packet arrival rate. We also derive the optimized check interval values for X-MAC and WiseMAC protocol, but left out the derivations in this thesis. Hence, Figure 3.7 presents the optimized check interval values calculated using mathematical expressions for all three protocols for a given traffic load. We see that WiseMAC protocol can have smaller check 


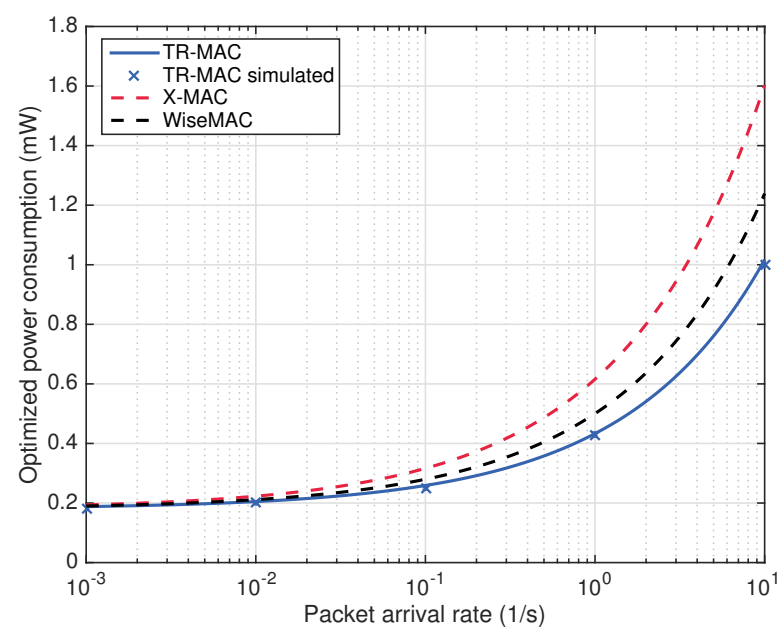

Figure 3.8: Unsynchronized link state: Power consumption using optimized check interval for varying packet arrival rate

interval, because the receiver node only needs to detect the preamble. On the other hand, TR-MAC and X-MAC need to have longer check intervals than WiseMAC. Afterwards, we use these optimized check intervals to calculate the energy consumption. We plug in the optimized check interval duration depending on the given packet arrival rate in the simulator so that the nodes can start with the optimized check interval from the beginning of the operation.

Next we calculate the overall energy consumption in the unsynchronized link state for a range of traffic arrival rates. In this case, the protocols use the previously calculated optimized check intervals. The results are given in Figure 3.8, where the simulation results for TR-MAC protocol matches with the analytical results. Confidence intervals are not drawn in this figure, because $95 \%$ of the values were smaller than $1 \%$ of the displayed value. We see that the TR-MAC protocol in the unsynchronized link state performs better than the reference protocols, even though the transmitter using TR-MAC uses double power than 


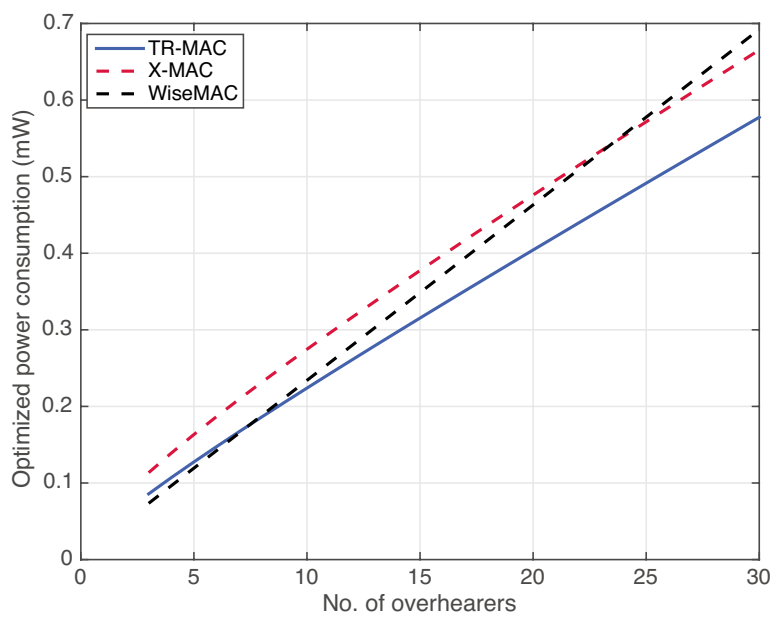

Figure 3.9: Unsynchronized link state: Energy with overhearers for packet arrival rate $=0.1$ packets $/$ second

other protocols. TR-MAC overhearers can return to sleep after receiving one preamble-data packet, effectively minimizing its periodic listening. On the contrary, WiseMAC has more energy consumption because the overhearers have to listen until the end of the preamble before deciding whether to receive the data packet or return to sleep.

In Figure 3.9, we represent the comparison of energy consumption for these three protocols for different number of overhearers in the system. We see that the TR-MAC protocol has less power consumption than the X-MAC protocol. The difference between the TR-MAC and the WiseMAC protocol decreases with a small number of overhearers, and increases with a large number of overhearers. The fact that the TR-MAC protocol saves energy for overhearing contributes towards the energy saving in the unsynchronized link state operation. 


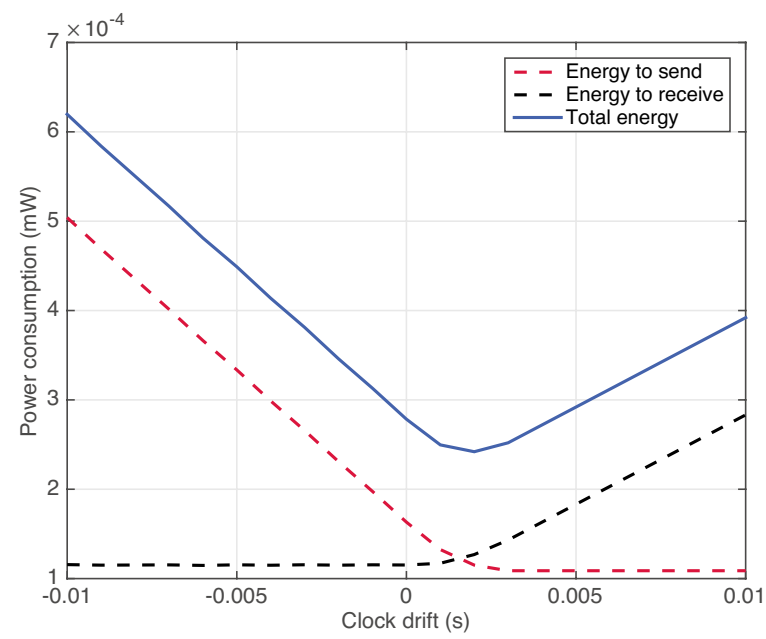

Figure 3.10: Synchronized link state: Power consumption for clock drift

\subsubsection{Synchronized link state}

The TR-MAC protocol in the synchronized link state effectively minimizing the preamble duration in transmitter side, since either the transmitter or the receiver follows each other's next wake up time. The energy consumption in the synchronized link state depends on the relative wake up time of the transmitter and receiver clocks, as presented in Section 3.4, Figure 3.3. Figure 3.10 represents the power consumption to transmit or receive in the synchronized link state of TR-MAC for a range of clock drifts. The clock drift of both the transmitter and the receiver are assumed to be uniformly distributed between $-30 \mathrm{ppm}$ to 30 ppm, and the time since last communication is assumed to be 50 s. Here we see that the total energy consumption does not reach its minimum value when the clock offset is zero. Rather the total energy consumption reaches its minimum value at a point with some positive clock difference, around .002 seconds in this particular case. This result indicates that waking up the receiver .002 seconds earlier than the transmitter would minimize the total energy consumption for this case, which was proposed in the optimization method in Section 3.4. Hence, 


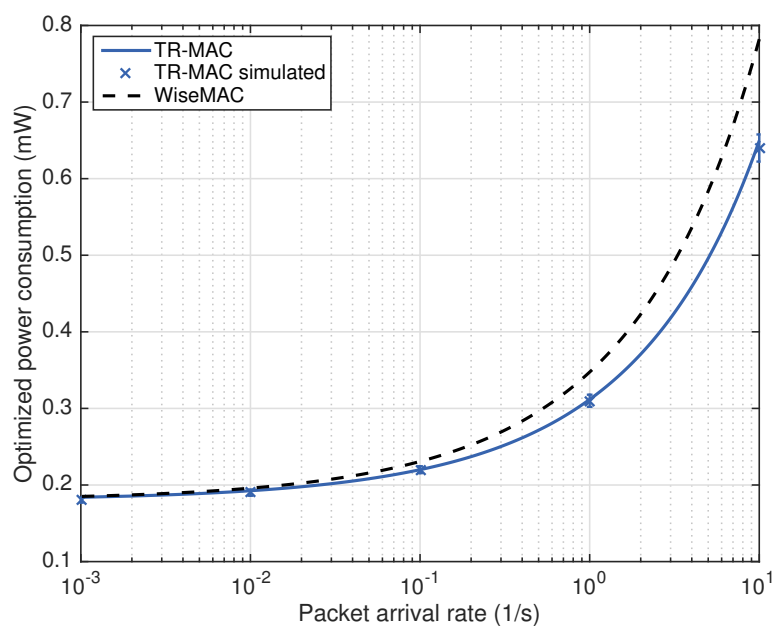

Figure 3.11: Synchronized link state: Power consumption for varying packet arrival rates

an intentional clock offset, $\delta$, can be introduced depending on the clock drift, $\theta$, and time since last communication, $l$, to wake up the receiver earlier than the transmitter for the future communication to optimize the energy consumption in the synchronized state of the TR-MAC protocol.

To model the synchronized link state for TR-MAC protocol with the optimization in the simulator, we take the clock drift as a random value from a uniform distribution for both the sender and the receiver node, and maintain the time since last communication between these nodes. Depending on these parameters, the nodes can determine the amount of the intentional clock offset needed to adjust their future wake up times. We also modelled the WiseMAC protocol for synchronized link state. The X-MAC protocol has been left out for the synchronized case, since it does not have any synchronized link state. Figure 3.11 presents the energy consumption for varying packet arrival rates calculated from both analytical models and simulation measurements for the optimized TRMAC synchronized link state, together with the analytical results for WiseMAC synchronized link state. We see that the simulation results confirm the analyt- 


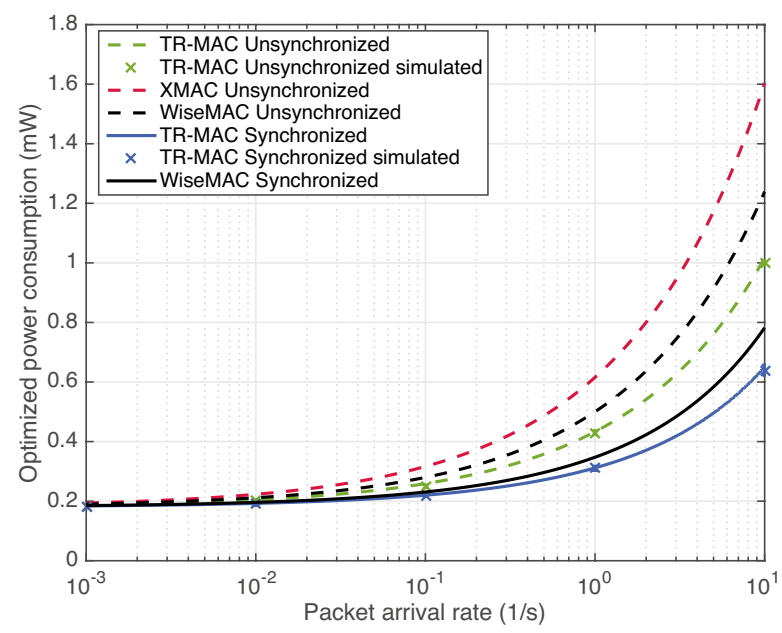

Figure 3.12: Unsynchronized and synchronized link state comparison

ical results for TR-MAC protocol. We find that optimized TR-MAC protocol consumes less energy than WiseMAC protocol in the synchronized link state. Unlike WiseMAC, the overhearers in TR-MAC are unable to overhear the communications in the synchronized link states, because the future communications in synchronized link states take place in different frequency offsets.

\subsubsection{Comparison of unsynchronized and synchronized link state}

The overall energy consumption for the TR-MAC, X-MAC and WiseMAC protocols for both the unsynchronized and the synchronized link state with respect to varying packet arrival rate is illustrated in Figure 3.12. As a result, this figure combines the results of the previously presented individual results for the unsynchronized and synchronized link states. The protocols use optimized check interval for individual traffic loads for the unsynchronized and synchronized link state. Here we see the TR-MAC protocol in the synchronized link state has better energy consumption than the unsynchronized link state compared to the X-MAC and WiseMAC protocol. Thus, the TR-MAC protocol tries to switch to 
the synchronized link state whenever possible. The fact that the TR-MAC protocol in the synchronized link state can optimally minimize the preamble-data and acknowledgement listen cycle duration to as small as possible contributes towards the overall energy saving for the protocol operation.

\subsection{Summary}

In this chapter, we present the TR-MAC preamble sampling protocol based on TR modulation for wireless sensor networks that utilizes the advantages of TR modulation. TR-MAC receiver can detect a very small preamble as the receiver architecture using TR modulation has fast signal acquisition and synchronization capability. TR-MAC transmitter takes this advantage, and appends small data packets ranging from very few bytes with the preamble, referred to as preamble-data. At the same time, the TR-MAC protocol minimizes the power penalty of the TR modulation in the synchronized link state using minimum preamble-data transmissions and listen iterations for a successful communication. Furthermore, the TR-MAC protocol can use multiple frequency offsets for simultaneous communications in the synchronized link state. The TRMAC protocol with its unsynchronized and synchronized link states has both transmitter-driven and receiver-driven communication possibilities based on the specific application requirement, or based on available energy on the node. As a result, the TR-MAC protocol can provide opportunities for energy-efficient routing in the network layer. The TR-MAC protocol analytical modelling and the simulation results are compared with the two reference preamble sampling protocols for a system of a realistic number of nodes. The results show that the TR-MAC protocol is energy-efficient, for both the unsynchronized and the synchronized link states. Furthermore, the TR-MAC protocol successfully mitigates the common energy wastage of WSN, namely idle listening, overhearing, control packet overhead, and collisions. Overall, TR-MAC is promising for energy-efficient communications for low power and low data rate applications of wireless sensor networks. 



\section{Traffic adaptation in MAC protocols}

MAC protocols for WSN require to satisfy certain performance requirements, although they are influenced by a number of constraints. Since MAC protocols are mostly application dependent, a specific MAC protocol tries to make a tradeoff among the important performance parameters based on the specific application requirements. The important performance parameters for the MAC protocols include energy-efficiency, delay per packet, throughput, etc.. A MAC protocol achieves energy-efficiency by employing low duty cycling for a transceiver. Duty cycling in the context of WSN MAC protocols means to turn off the transceiver to save energy. However, lower delay per packet or higher throughput requires comparatively higher duty cycling for a transceiver to be able to deliver higher number of packets. Therefore, a MAC protocol that prioritizes energy-efficiency, often has to sacrifice the quality of service parameters of delay per packet and throughput [67]. As a result, these protocols often fail to adapt to higher traffic load, since they are designed to satisfy lower traffic load with low duty cycles.

In Chapter 3, we discussed the energy-efficient TR-MAC protocol, which is suitable for low power, low data rate, and low duty cycle WSN scenarios. However, low data rate cannot always be maintained in wireless sensor networks. Due to application specific requirements, a WSN often has to deal with eventdriven scenarios, where a sudden event rapidly increases traffic load within the network. In this chapter, we present a traffic adaptation mechanism in order to provide responsiveness to traffic rate variations for the TR-MAC protocol, 
which is based on the published paper [22]. This mechanism increases throughput, and decreases packet delay while maintaining energy-efficiency with very limited extra information exchange among the sensor nodes in the network. This chapter answers the research question 3 ("How can traffic-adaptive behavior be attained while maintaining energy-efficiency?"). The contributions presented in this chapter are: i) we present a traffic-adaptive duty cycle adaptation algorithm for the TR-MAC protocol for varying traffic while maintaining energy-efficiency, ii) we combine the traffic-adaptive duty cycle adaptation algorithm together with a request-based traffic adaptation method to improve throughput and reduce per packet delay, iii) we compare the TR-MAC protocol using this traffic adaptation approach with two reference protocols in the $\mathrm{OMNeT}++$ simulator using the MiXiM simulation framework.

This chapter is organized in the following manner: Section 4.1 presents the motivation of this work. After that, Section 4.2 discusses the related work regarding traffic adaptation in wireless sensor networks. The traffic adaptation techniques of the TR-MAC protocol is presented in Section 4.3. We present and analyze the results in terms of QoS parameters and energy-efficiency in Section 4.4. Finally, Section 4.5 provides summary.

\subsection{Motivation}

Energy-efficiency is an important criterion for MAC protocols to operate in wireless sensor networks. To achieve energy-efficiency, MAC protocols employ transceiver duty cycling to keep the sensor node's radio transceiver in sleep mode as much as possible to minimize the overall energy consumption. Therefore, the protocols that use transceiver duty cycling achieve energy-efficiency as trade off for important performance parameters, for example, throughput or delay. As discussed in Chapter 2, asynchronous preamble sampling protocols tend to be the most energy-efficient category of MAC protocols for low data rate traffic scenarios because they allow the nodes to sleep most of the time without network wide wake up synchronization [6]. Thus, the nodes can independently wake up periodically to check any ongoing activity in the channel. The duration 
between two consecutive periodic duty cycles is referred to as the check interval. For a successful communication, the sender node has to transmit a preamble of check interval duration of the potential receiver node in order to reach the receiver node when it is awake. Therefore, there has to be a maximum value for the check interval to be known in the WSN, even though one node in a WSN can have a smaller check interval duration than the global maximum value. However, the basic preamble sampling protocols lack traffic-adaptivity due to their rigidity to support delay sensitive applications. Hence, these protocols achieve energy-efficiency at the expense of higher per packet delay and lower throughput in the presence of an event-driven higher traffic load scenario, since improving both the energy-efficiency and the quality of service parameters at the same time is difficult [67].

We have proposed a preamble sampling based energy-efficient MAC protocol for WSN in Chapter 3 based on the publications in $[19,20,21]$. This new MAC protocol for WSN is called the TR-MAC protocol and has two operating states: i. unsynchronized link state, and ii. synchronized link state. In the unsynchronized link state, the TR-MAC protocol uses incoming traffic load to optimize the check interval in order to achieve energy-efficiency. However, one node has to measure several parameters in order to use the analytical model to optimize the check interval. Furthermore, one node does not have any knowledge about its surroundings in the beginning. Moreover, some parameters might change during the network lifetime. Thus, at a certain point in time, one node might miscalculate the network parameters, e.g., packet arrival rate, or the number of neighbors, which in turn can influence the node to miscalculate the optimized check interval using the analytical model. Furthermore, power consumption is higher in the unsynchronized link state, because the sender has to take the responsibility of reaching the receiver by sending data packets and wait for the acknowledgements for a maximum of the system level check interval duration. That is why the nodes try to make pairs and move to the synchronized link state after agreeing about a future time instance and communicating frequency offset within a pair. Therefore, the pair of nodes is successfully offloaded from the default frequency offset, because they can communicate at a different frequency 
offset in the future using the synchronized link state.

In the synchronized link state, one node allocates an extra listen cycle and frequency offset to its neighbor pairs to communicate in future. The difference between two of these extra listen cycles is also the same as the check interval. This approach enables the sender to communicate exactly when the receiver wakes up. In this way, this approach saves the power consumption in the protocol operation. However, the throughput and the delay per packet remain constant in this approach, which is not a desirable situation in the presence of higher event-driven traffic. If the packet arrival rate in the sender exceeds the communication frequency with its receiver pair, then the packets experience queuing delay at the sender node. For example, if two nodes in a pair agree to communicate after every one second, and the packet arrival rate exceeds one packet per second, then the sender node in this pair cannot deliver the packets at a rate of more than one packet per second to its receiver pair. Moreover, if one node adopts a traffic-adaptive behavior by increasing its duty cycle using a particular frequency offset, then its neighbors cannot take the benefit of these extra listen cycle. The reason is, one node communicates with its neighbor pairs using different frequency offsets in the synchronized link state. As a result, maintaining energy-efficiency and at the same time performing better in the quality of service parameters with minimum overhead is very difficult for a preamble sampling based asynchronous MAC protocol to deal with variations in offered traffic load for event-driven scenarios. Therefore, in this chapter, we investigate the design of a traffic-adaptive approach to provide better performance for quality of service parameters for the synchronized link state of the TR-MAC protocol with minimum amount of extra information exchange, and to minimize overall energy consumption in a WSN.

\subsection{Related work}

Preamble sampling protocols are the most energy-efficient category of MAC protocols, as discussed in the Chapter 2. These protocols use low duty cycle to achieve energy-efficiency, thus often suffers in the delay or throughput pa- 
rameters. In Chapter 3, we presented a preamble sampling based TR-MAC protocol to achieve energy-efficiency in WSN. In this section, we discuss existing literature about preamble sampling protocols that attempt to adapt to variable traffic load. To increase the packet receive rate based on high traffic load scenario, some protocols enable the nodes to learn about the increasing traffic load based on thresholds on packet arrival rate, or overhearing other ongoing communications. In some cases, the node informs its neighbors about its updated duty cycle to enable the neighbors to send packets at a higher rate. Few other techniques use specific request from the potential neighboring sender nodes to indicate that the receiver node need to increase its duty cycle. The papers [67] and [6] provide a survey of preamble sampling protocols from the delay and throughput perspective.

The approaches dealing with adopting traffic-adaptive behavior using the duty cycle based preamble sampling technique involves increasing duty cycle of a node to accommodate the increasing traffic. The authors in [59] propose an extension of the basic B-MAC protocol [38], where one node increases its duty cycle after realizing the increase in incoming packet rate. One node informs its neighbors about the updated duty cycle only when it communicates with other nodes at a future time. All the nodes maintain a list of the duty cycle of every neighbor nodes, and this list gets updated after overhearing a communication. The overhearing technique is not suitable for protocols with very low duty cycling, specially if the communications take place using different frequency offsets, like our TR-MAC protocol. The BoostMAC protocol [68] uses a technique, where one node increases its duty cycle in multiplicative steps after realizing about the increasing traffic, and decreases its duty cycle in additive steps when the traffic decreases. In this case, the potential senders decide the preamble length based on their own traffic rate. As a result, there is a probability that the packet may need to be retransmitted, because the intended receiver node could be sleeping when the packet was sent. Another traffic-adaptive protocol MaxMAC [25] is built on the WiseMAC [40] synchronized link state operation, by which one node doubles its base duty cycle based on a threshold of incoming packets towards it. This protocol uses three states and three 
thresholds, where the crossing the first two threshold leads one node to a higher state with doubling the duty cycle, and crossing the third threshold leads one node to operate in CSMA. The LWT-MAC protocol [69] employs a mechanism to operate in CSMA to increase the throughput, when the traffic load in the network increases. This protocol allows all the overhearer nodes to send packets to any nodes with a shorter preamble just after the previous packet transmission was completed. Using CSMA, the nodes are always active without having any sleep time. Thus, the energy-efficiency is completely sacrificed when the nodes in a protocol use CSMA.

The X-MAC [39] protocol enables the nodes to select their check interval based on their own estimation of traffic load. However, there has to be a network-wide maximum check interval for the sender node for the maximum duration of the preamble and acknowledgement listen duration, like the TR-MAC protocol. Furthermore, X-MAC generally has a high per packet overhead, because of operating only in the unsynchronized link state. The protocol SCP-MAC [43] proposes a traffic load based duty cycle adaptation, where the receiver node adds few extra listening cycles just after receiving a packet from the sender. Once these extra listening cycles are not used, the receiver node deletes them, and goes back to the basic preamble sampling. Another protocol [70] proposes an asymmetric additive duty cycle control (AADCC) to increase the sleeping time of a node when five consecutive packets are received, and to decrease the sleeping time after one packet miss. This protocol thinks that receiving five packet consecutively means that the channel contention is low; thus, the nodes can sleep for a longer time. However, in actual scenario, receiving five consecutive packets could also mean that the traffic load is increased. DuoMAC [71] provides runtime parameter adaptation targeting heterogeneous traffic applications to balance energy-minimization with delay-minimization by adaptively switching between two states according to the dominating traffic in the network.

There are some protocols where one sender node experiencing higher traffic load initiates a request to its potential receiver node to increase its duty cycle. The authors in WiseMAC [40] propose a technique to send burst packets from a 
sender to a receiver after a successful packet reception without waiting for the next listen cycle. In this case, one sender assigns a bit to indicate to the receiver that more data packets are coming towards the receiver. After receiving this request from its sender pair, the receiver node changes its duty cycle pattern, and continues to listen after sending the acknowledgement back to the sender. This approach allows the delivery of many packets in a burst from a single sender to a single receiver at once without waiting for the next periodic duty cycling. Furthermore, this approach reduces the end-to-end delay for burst traffic. The authors in WiseMAC More bit extension[57] propose an enhancement of WiseMAC with an overhearing technique and stay awake promise. Using this technique, one receiver node stays awake for a longer time after receiving a request from a sender node. Another node, which wants to send to the same receiver node, overhears to an ongoing transmission and can send data packets right after the first node finishes its communication to the same receiver node. Another similar technique is used in BEAM [58], where the sender marks a bit in the data packet header to signal the receiver to double its duty cycle. As a result, the sender can effectively shorten its preamble duration from the maximum one, hence can increase throughput. The pQueueMAC protocol [72] uses synchronization based preamble sampling for lower traffic load, but dynamically allocates time slots based on TDMA for bursty traffic.

In Chapter 3, we have proposed the TR-MAC protocol that operates in the synchronized state in pairs, where different pairs communicate using different frequency offsets and a time instance. Therefore, one node can communicate with at most one other pair in the synchronized link state for a particular listen cycle using a specific frequency offset. Hence, one node adopting a trafficadaptive strategy by increasing its duty cycle using a particular frequency offset does not help its neighbors to take the benefit of this extra listen cycle, because the neighbors are more likely to use different frequency offsets to communicate with this node in the synchronized link state. Furthermore, one node cannot overhear any ongoing communication, unless they are using the same frequency offset. As a result, the traffic-adaptive approaches that uses overhearing technique to extend the listen cycle of a node to deliver more 
packets to a particular receiver node does not work with the TR-MAC protocol. Therefore, based on the literature studies, in this chapter, we investigate on achieving traffic-adaptive behavior while maintaining energy-efficiency using the synchronized link state of the TR-MAC protocol, which is suitable for varying traffic load scenarios to increase throughput and decrease delay with minimum amount of extra information exchange.

\subsection{Traffic adaptation in the TR-MAC protocol}

The energy-efficient TR-MAC protocol was originally designed for low data rate, and low duty cycle WSNs. Therefore, the MAC protocol has to adapt to the changes in traffic caused by a sudden event-driven scenario within the network. In the occurrence of these kinds of scenarios, the packets are required to be disseminated fast while maintaining the energy-efficiency. In this section, we provide a traffic-adaptive duty cycle adaptation algorithm, which is capable of disseminating packets at a higher rate in the presence of an event-driven scenario.

\subsubsection{Traffic-adaptive duty cycle adaptation}

In this subsection, we propose a traffic-adaptive duty cycle adaptation algorithm to be used in the preamble sampling based TR-MAC protocol in the synchronized link state. In the synchronized link state for the TR-MAC protocol, a transmitter and a receiver pair wakes up at a previously agreed time instance and frequency offset to communicate in an energy-efficient manner, as described in Chapter 3. The receiver node in the pair wakes up at a previously agreed time instance using a particular frequency offset to check any channel activity, and the sender node of the pair also transmits in the same time instance using the same frequency offset. In this way, the sender node can optimally minimize the preamble-data and acknowledgement listen cycle duration to as small as possible, and the receiver can minimize its idle listening duration. The rate at which packet communication can take place in the synchronized link state 
is limited to the frequency of the agreed upon communication cycles of this pair of nodes. In case of an event-driven scenario, the sender node experiences increased traffic load, and it needs to deliver packets frequently to its receiver pair. If the packet arrival rate in the sender exceeds the communication frequency with its receiver pair, then the packets queue up in the sender node. As a result, the packets in the sender node experience extra delay. To solve this problem, we propose a traffic-adaptive duty cycle adaptation algorithm where a pair of nodes adapts their communication frequency based on the increasing or decreasing traffic rate while maintaining the energy-efficiency characteristics of the preamble sampling protocols.

According to the traffic-adaptive duty cycle adaptation algorithm, a potential receiver node increases its duty cycle by waking up at a higher rate for increasing traffic, and decreases its duty cycle by removing the extra listen cycles for decreasing traffic. In order to take the advantage of these extra listen cycles of the receiver, the sender node has to send packets when the receiver node wakes up. We propose a traffic-adaptive duty cycle adaptation algorithm, where the sender node uses the same algorithm to estimate the extra listen cycles of its receiver pair, and can send packets targeting those extra listen cycles of its receiver pair. In this way, one receiver node does not need to inform its current wake up schedule to its pairs, even though it has increased its listen cycles based on increasing traffic rate that it experiences from its sender pair. The trafficadaptive algorithm has several states, where a lower state means infrequent communication cycles, and a higher state means frequent communication cycles. The pair of nodes can move to a higher or lower states based on the experienced traffic load.

The receiver node realizes that the traffic load is increasing when it receives packets consecutively for a number of its listen cycles. Similarly, the sender node realizes that the receiver node received all the data packets, when it receives consecutive acknowledgments for all the data packets it sent to the receiver node. Therefore, we define a state increase parameter, $K$, for the number of consecutive packet reception without any interruption, and another state decrease parameter, $L$, for the number of consecutive empty listen cycles 


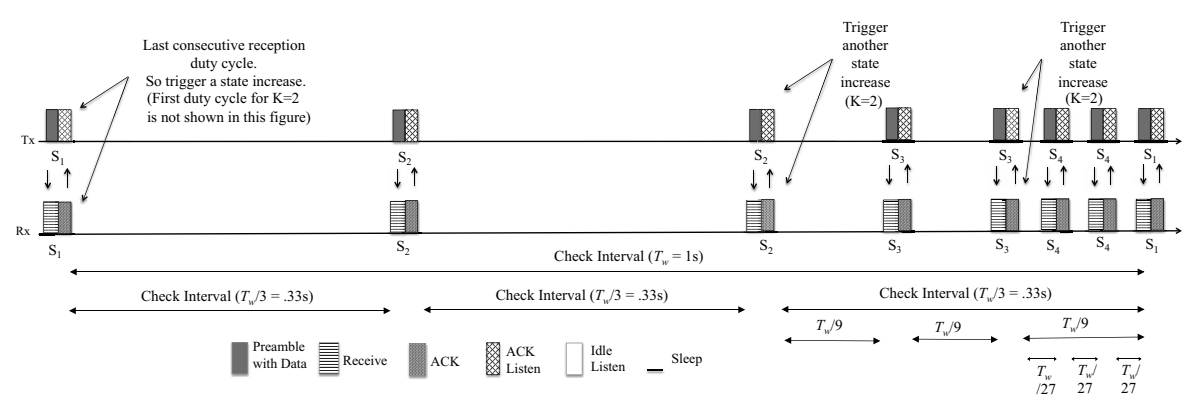

Figure 4.1: Traffic-adaptive duty cycle adaptation for TR-MAC protocol

without any interruption. The state increase parameter, $K$, and the state decrease parameter, $L$, triggers the shifting among the higher or lower states, as they indicate the current traffic load. The total number of states is defined by the parameter $R$, and the operating states are defined by the parameter $S$. In a higher state, extra listen cycles are be added. The time instances of these extra listen cycles are determined by dividing the base check interval, $T_{w}$, with the listen cycle time decider parameter, $M$. This listen cycle time decider parameter is defined as $M=\left\{M_{1}, M_{2}, \ldots, M_{R}\right\}$, where $M_{i}=(R-1)^{i-1}$ for $(1 \leqslant i \leqslant R)$. For example, total number of states parameter value, $R=4$, results in four operational states $S=\left\{S_{1}, S_{2}, S_{3}, S_{4}\right\}$ with listen cycle time decider parameter values, $M=\left\{M_{1}, M_{2}, M_{3}, M_{4}\right\}=\left\{3^{0}, 3^{1}, 3^{2}, 3^{3}\right\}=\{1,3,9,27\}$.

Figure 4.1 illustrates the state based mechanism for the traffic-adaptive duty cycle adaptation algorithm, and the decision making process to switch between these states together with the time instances of the extra added listen cycles using a timeline for state increase parameter, $K=2$. That is, consecutive two packets reception triggers a state increase. Initially, the protocol operates in the first starting state $S_{1}$ with the default base check interval, $T_{w}$. The first duty cycle $S_{1}$ in the figure represents the last duty cycle of the consecutive packet reception. When one receiver node receives packets in $K$ consecutive listen cycles without any interruption while operating in starting state $S_{1}$, it triggers a state increase operation. Then that receiver node needs to move to the immediate higher state of $S_{2}$ by adding extra wake up cycles at $T_{w} / M_{2}$ time instances to accommodate 
higher traffic, instead of waiting until the base check interval, $T_{w}$. This same procedure is repeated to move to state $S_{3}$ by adding extra listen cycles at $T_{w} / M_{3}$ time instances, and then to state $S_{4}$ by adding extra listen cycles at $T_{w} / M_{4}$ time instances to accommodate even higher traffic. As a result, the receiver node can quickly reduce its check interval to adapt to the increasing traffic by a factor of the listen cycle time decider parameter, $M$. The sender node also uses the same algorithm, and triggers a state increase operation depending on receiving $K$ consecutive acknowledgements without any interruption. Consequently, the sender and the receiver pair can increase their communication frequency depending on the increasing traffic load without any extra information exchange. We present an analysis about different parameter values for the state increase parameter, $K$, and state decrease parameter, $L$, in Subsection 4.4.4. This trafficadaptive duty cycle adaptation algorithm is presented in Algorithm 4.1.

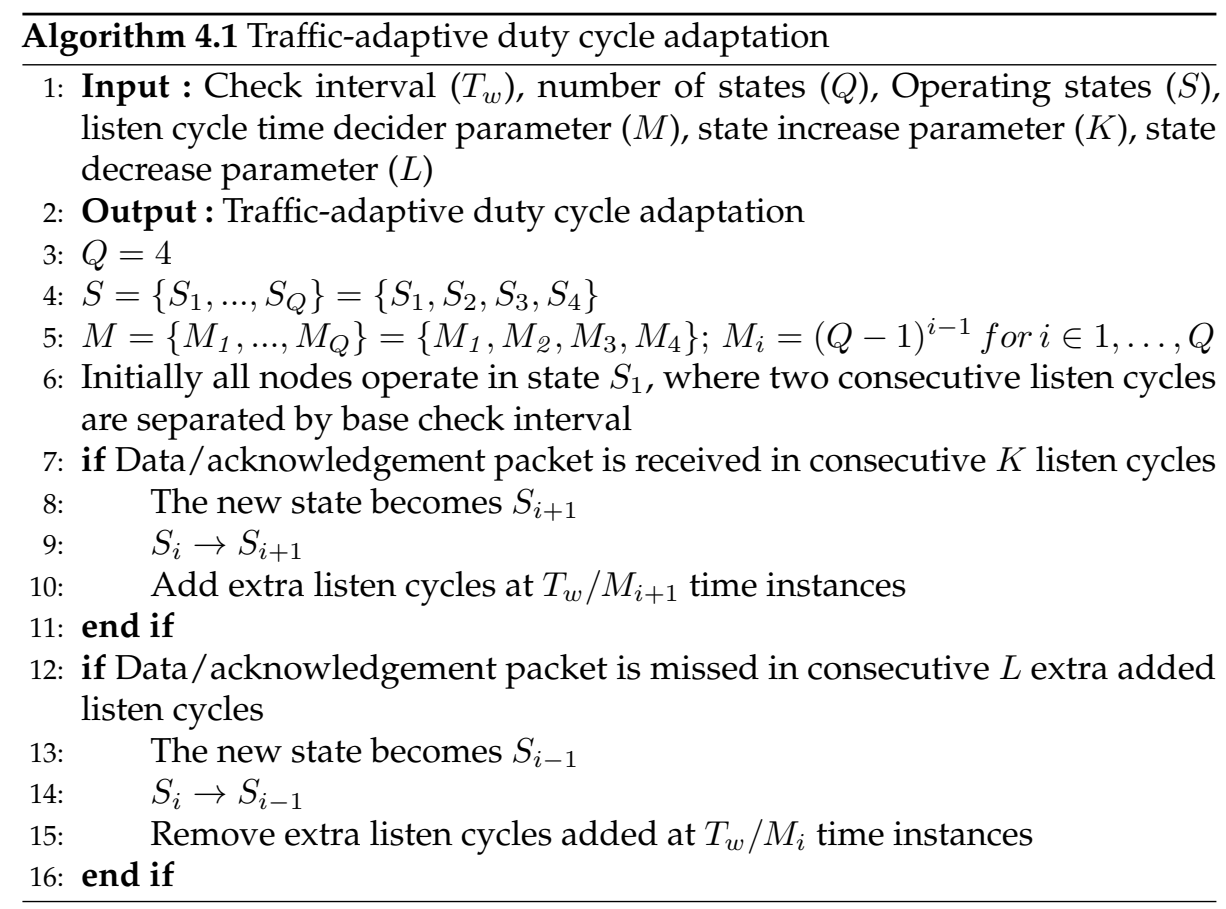


The protocol adapts to the decreasing traffic rate by deallocating the added extra wake ups, and moving to a lower state, when it does not receive any packets in $L$ number of consecutive extra added listen cycles without any interruption. Hence, the node deallocates the extra listen cycles added previously, and moves to the immediate lower state. For example, if a receiver node is operating in state $S_{3}$, and does not receive packets in $L$ consecutive extra added listen cycles, then this node deallocates the previously added extra listen cycles, and ramps down to state $S_{2}$. After successfully delivering the instantaneous increased traffic load, the receiver node eventually moves down to initial base state $S_{1}$ to continue waking up after base check interval by deallocating all the previously added extra listen cycles. Correspondingly, the sender node also deallocates the extra packet sending operations at a higher rate depending on receiving no acknowledgements for $L$ number of consecutive listen cycles without any interruption, and moves to a lower state. Later in Section 4.4.4, we further investigate about choosing the optimum values for the state increase parameter $K$ to allocate extra listen cycles to move to a higher state, and the state decrease parameter $L$ to deallocate the previously allocated extra wake ups to return back to a lower state.

The advantage of this traffic-adaptive duty cycle adaptation algorithm is that both the sender and receiver pair can use the same algorithm in the same manner to adapt to the change of the traffic load. As a result, one pair of nodes does not need to exchange any information to inform its pair node about the allocation of the extra duty cycle time instances in case of increasing traffic load, or about the deallocation of the previously added extra wake up cycles. As a result, this algorithm does not bring any overhead about network-wide communication within the WSN. One receiver node can increase the communication frequency with more than one sender node by increasing the communication frequency in each pair. In this way, this duty cycle adaptation mechanism can increase the packet receive rate for a particular receiver node, when it is receiving data from more than one sender node. However, increasing the communicating frequency with more than one sender pair is limited to the available non-overlapping time instances that one receiver node can offer to its sender pairs. In case of 
overlapping time instances, the receiver node needs to provide priority to its sender pairs. By using this traffic-adaptive duty cycle adaptation algorithm, the delay per delivered packet is expected to decrease, and the throughput is expected to increase. The impact of packet loss on this traffic-adaptive duty cycle adaptation algorithm is presented in Subsection 4.3.3.

\subsubsection{Duty cycle adaptation combined with request-based burst packet transfer}

In the literature, we find a request-based burst packet transfer methodology that enables a node to send multiple packets from its queue destined to a single node. In this case, the sender sets an one bit flag, called More Bit, to request the receiver to receive more packets at this moment without waiting for the next listen cycle of the receiver. Receiving this request by setting the More Bit in the header section of the data packet indicates the receiver that one more data packet is coming towards the receiver. Thus, the receiver prepares to receive the next data packet, after sending the acknowledgement packet for the previous received data packet. On the other side, the sender node receives the acknowledgement, then sends the next packet from its queue, and waits for the acknowledgement from the receiver. Once again the receiver sends an acknowledgement back to the sender after reception of the new data packet. In case the sender wishes to send more data packets to the same receiver, it repeats the same procedure by setting the More Bit, and the receiver individually acknowledges each data packet. When the sender has no more packets in its queue to send to the same receiver, then it resets the More Bit to zero to the last data packet of the burst, which indicates that the receiver node can return to sleep after sending the last acknowledgement. This request-based adaptation mechanism is illustrated in Figure 4.2 for the base check interval.

This request-based burst packet transfer technique increases throughput significantly between a communicating pair of nodes, because the sender node does not need to wait for the next duty cycle of the receiver node to transmit packets. Consequently, this request-based burst packet transfer reduces the queueing 


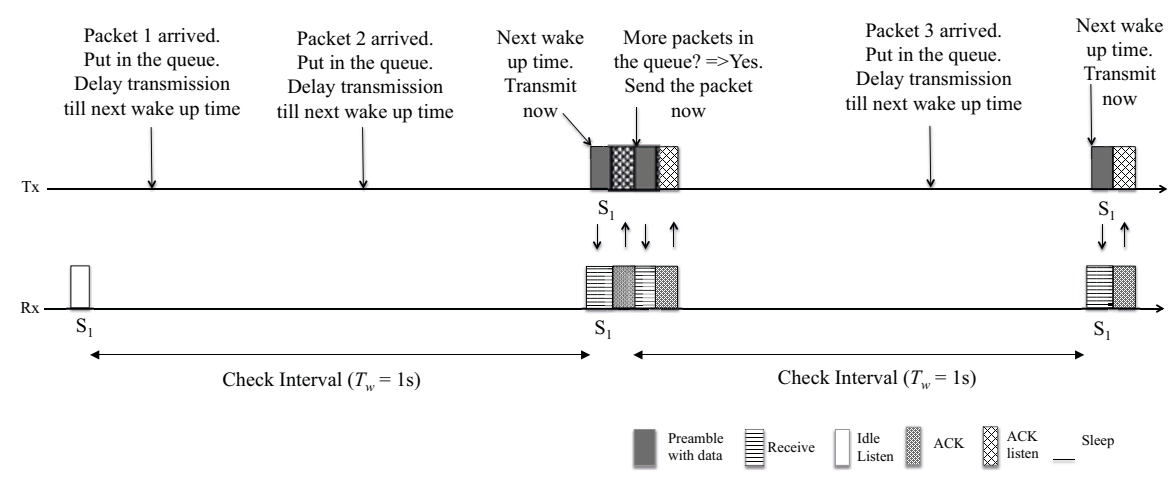

Figure 4.2: request-based burst packet transfer

delay per packet at the sender node. The request-based burst packet transfer method is useful, however, it is limited by the queue size or the maximum burst size at the sender node. Furthermore, this request-based strategy does not increase the communication frequency within the pair of nodes in the event of higher traffic, rather continues to have listen cycles after the base check interval. As a result, the benefits of this request-based burst traffic method gets limited. Therefore, we propose to combine this request-based burst packet transfer method together with our traffic-adaptive duty cycle adaptation method. As a result, the sender node in a pair can send a burst of packets to its receiver pair for a single listen cycle. This combination of two traffic-adaptive methods enable a pair of nodes to communicate at a much higher rate compared to a single approach. Hence, a combination of these two traffic-adaptive approaches is expected to provide better performance in the quality of service parameters by increasing the throughput and decreasing the delay per packet.

\subsubsection{Dealing with packet loss}

In this subsection, we explain the consequence of packet loss on our trafficadaptive algorithms. A lost packet has to be retransmitted. Furthermore, the traffic-adaptive algorithm needs to work in the presence of packet loss scenarios. 
We present the effect of packet loss on the traffic-adaptive duty cycle adaptation algorithm, and on the request-based burst packet transfer algorithm separately.

\subsubsection{Traffic-adaptive duty cycle adaptation algorithm}

In this subsection, we explain the working process and consequences of the traffic-adaptive duty cycle adaptation algorithm in the event of packet loss. The pair of nodes works using the synchronized link state of the TR-MAC protocol. Therefore, the sender wakes up to send a packet to its receiver pair at a previously agree time instance and frequency offset. The sender makes only one attempt to send packet to the receiver for its listen cycle, because of the optimization of the synchronized state discussed in Chapter 3. A packet loss is always followed by a retransmission. In most of the cases, the receiver and the sender pair follow each other by operating in the same state. In some cases, there is a possibility of having a mismatch in the operating states of the receiver and the sender pair, but eventually they converge to operate in the same state. There are four scenarios possible, namely i) no packet exchange, ii) packet exchange with no loss, iii) data packet is lost, and iv) acknowledgement is lost. We explain these four scenarios below:

i. No packet exchange: In the case of no packet exchange, both the receiver and the sender operate in the basic synchronized link state with the duty cycles separated by the base check interval. Therefore, both of the nodes in a pair operate in the same state without any mismatch.

ii. Packet exchange with no loss: For packet exchange with no loss, both the receiver and the sender move to a higher state or to a lower state at the same time without any mismatch in operating states. As a result, no extra cost involved in the traffic-adaptive operation.

iii. Data packet is lost: For the loss of a data packet, the receiver does not send an acknowledgement back to the sender node. Consequently, the sender also does not receive an acknowledgement from the receiver node. Therefore, both the receiver and the sender experience similar behavior of missing a data packet or an acknowledgement. The state increase parameter, $K$, is reset to zero in the receiver node for a single data packet loss. Correspondingly, the sender node 
also resets the state increase parameter, $K$, to zero, since it does not receive an acknowledgement in the event of a data packet loss. As a result, both the nodes experience similar amount of delay to move to the next higher state.

On the other hand, missing the data packets for consecutive $L$ number of listen cycles triggers the receiver to move to a lower state. As the receiver does not send any acknowledgements packet in case of a data packet loss, the sender misses the corresponding acknowledgement packets. Missing the acknowledgement packets for consecutive $L$ number of listen cycles also triggers the sender to move to a lower state. As a result, both the receiver and the sender follow each other in converging to operate in the same state in the event of consecutive data packet losses. Hence, the performance may suffer, but the state of the receiver and the sender will be consistent.

iv. Acknowledgement is lost: For the loss of an acknowledgement packet, there is a possibility of having a mismatch in the operating states of the receiver and sender. Considering that initially both the receiver and the sender node in a pair are operating in the same state. In the acknowledgement loss case, the receiver sends an acknowledgement after receiving the data packet, which is not received by the sender. As a result, receiving consecutive $K$ packets triggers the receiver to go to a higher state by adding extra wake up cycles. However, the state increase parameter, $K$ is reset to zero for a single acknowledgement packet loss. Hence, the sender node does not trigger to go to a higher state, because it did not receive the acknowledgements for consecutive $K$ listen cycles as the corresponding receiver node. This results in one state mismatch in the state based operation between the receiver and the sender, where the receiver may operate in a higher state while the sender may operate in a lower state in the event of an acknowledgement packet loss.

The consequence of this mismatch operation is that the receiver has extra listen cycles, since it is operating in a higher state. However, the sender does not send packets targeting for those extra listen cycles, because it is operating in a lower state. The extra cost of this mismatch is the few extra listen cycle operations performed by the receiver. The converging operation takes place, when the receiver realizes that it is not receiving packets for those extra added listen 
cycles by operating at a higher state. Therefore, the receiver removes those extra added listen cycles, and moves to a lower state after missing data packets in consecutive $L$ listen cycles. As a result, both the receiver and the sender converge to the same operating state at an expense of few extra listen cycle operations performed by the receiver.

For a special case, the corresponding sender may trigger to go to the lower state after missing acknowledgements in consecutive $L$ listen cycles. In this case, the receiver and the sender may be more than one state apart. For this case, the receiver needs to perform two state decrease operations. The state decrease parameter $L$ needs to provide the stability in an operating state in order to prevent a node to quickly trigger a state decrease operation. This in turn enables the pair of nodes to be only one state apart, from which it is easier to converge to the same state.

Because of the acknowledgement packet losses, the opposite scenario of the sender operating in a higher state and the receiver operating in a lower state is impossible to happen. Therefore, the event where the sender is sending frequently while operating at a higher state and the receiver is waking up infrequently to receive those packets is also impossible to happen.

We considered the effect of consecutive acknowledgement packet loss on our algorithm in this subsection. We have found that the traffic-adaptive duty cycle adaptation algorithm works in the presence of packet loss with few consequences. The algorithm suffers in performance for the data packet loss, but the receiver and the sender converge quickly to the same operating state. For the acknowledgement packet loss, the receiver sometimes may operate in a higher state than its corresponding sender pair, but the receiver converges to the same lower state of the sender after some time at an expense of few extra listen cycle iterations.

\subsubsection{Duty cycle adaptation combined with request-based burst packet transfer}

The packet loss scenario with request-based burst traffic transfer is explained with two possible scenarios, namely i. loss of the first packet of the burst, ii. loss 
of an intermediate packet of the burst.

i. Loss of the first packet of the burst: Missing the very first data packet of the burst marks the end of that burst for the receiver. Similarly, missing the very first acknowledgement packet marks the end of that burst for the sender. Receiving the first packet of a burst indicates a successful reception in the consecutive packet reception parameter, $K$, for the duty cycle adaptation algorithm. Therefore, missing the first data packet or the first acknowledgement packet of the burst affects the traffic-adaptive duty cycle adaptation algorithm, since missing the first packet indicates one packet loss or one acknowledgement loss in the consecutive packet reception for the traffic-adaptive duty cycle adaptation algorithm.

ii. Loss of an intermediate packet of the burst: When an intermediate data packet of a burst is lost, the burst packet transfer has to be stopped. In this case, the receiver does not send the acknowledgement back to the sender pair, since it did not receive the data packet. As a result, the sender cannot send the rest of the packets from its queue, because it is not sure whether the receiver will listen to receive the next packet. The loss of an intermediate packet within the burst does not have any impact on the consecutive packet reception parameter of the traffic-adaptive duty cycle adaptation algorithm.

On the other hand, the burst packet transfer has to be stopped for an intermediate acknowledgement packet loss. In this case, the receiver continues to receive for one more data packet duration before marking a packet loss, because it received the last data packet. However, the sender does not receive the acknowledgement, and immediately stops the burst packet transfer.

\subsection{Results and analysis}

In this section, we investigate the performance of our proposed traffic-adaptive duty cycle adaptation algorithm combined with request-based burst packet transfer method for the TR-MAC protocol operating in the synchronized link state. We implement the TR-MAC protocol with traffic-adaptive duty cycle adaptation combined with request-based burst transfer in the OMNeT++ simulator 
using the MiXiM simulation framework. We use a pair of nodes to experiment with the traffic-adaptive duty cycle adaptation algorithm combined with the request-based adaptation. We consider a queue length of 20 at the sender node, and a Poisson arrival stream of packets. We also implement and compare our protocol with the preamble sampling protocols X-MAC [39] and WiseMAC [40]. We choose X-MAC as it is a basic preamble sampling protocol, and only operates in the unsynchronized link where nodes do not remember each other's wake up time. We choose WiseMAC because of its request-based burst packet transfer approach, and its capability to operate in the synchronized link state, which is similar to the TR-MAC protocol. We evaluate the protocol performance using system parameters mentioned in Chapter 3 for the transmission power, and packet duration for these protocols. We use a basic check interval for all the protocols, and gradually increase the packet arrival rate to observe their performance with respect to traffic-adaptive behavior. We compare these protocols in terms of throughput, delay per packet, and energy-efficiency. Afterwards, we present a parameter analysis for our proposed traffic-adaptive duty cycle adaptation algorithm. At the end of this section, we provide an analysis of the performance implications of packet loss for our proposed algorithm.

\subsubsection{Throughput}

The throughput comparison is presented in Figure 4.3 using logarithmic scale for varying traffic load with 1 second base check interval with 95\% confidence interval. We increase the Poisson traffic arrival rate at the sender node, and the sender node delivers the packets to its receiver pair. The throughput is calculated by dividing the total number of delivered packets by the total simulation time. The TR-MAC protocol, and the WiseMAC protocol operates in their respective synchronized link state, where the pair of nodes communicates with a previously agreed time instance. The X-MAC protocol does not have any synchronized link state. The TR-MAC protocol uses 4 states, where the receiver moves to a higher state depending on the number of consecutive packet reception in consecutive listen cycles, and the sender pair moves to a higher state depending on the consecutive acknowledgement receptions in consecutive ac- 


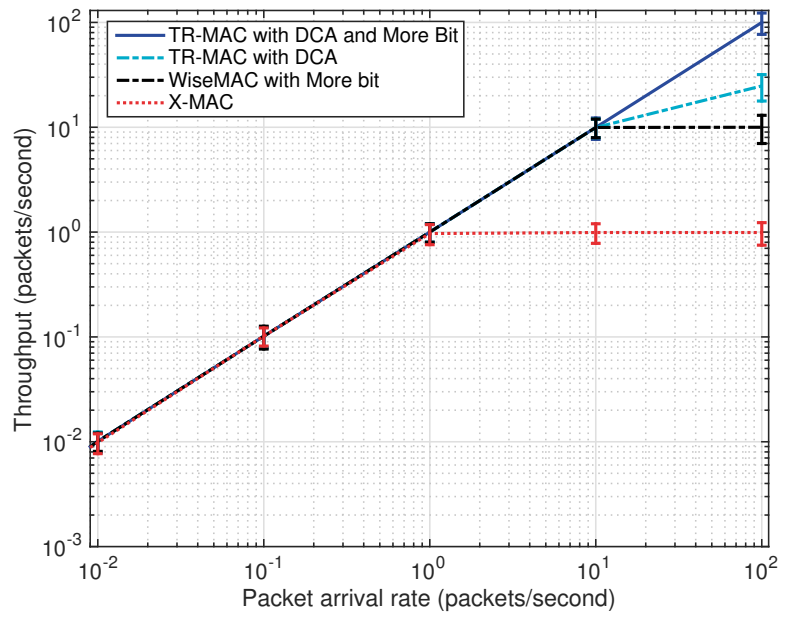

Figure 4.3: Throughput with varying traffic

knowledgement reception cycles. The state increase parameter $(K)$ determines whether one node will move to a higher state by adding extra listen cycles based on consecutive packet reception. Correspondingly, the state decrease parameter $(L)$ determines whether one node will move to a lower state by removing the previously adding extra listen cycles based on consecutive packet miss. We set the state increase parameter $(K)$ to 5 consecutive packet reception, and the state decrease parameter, $L$ to 10 consecutive packet miss. The reasons for choosing this parameter combination is analyzed in Subsection 4.4.4.

Here we observe that the TR-MAC protocol with the combination of duty cycle adaptation (DCA) together with request-based More Bit provides significantly higher throughput as the traffic load increases compared to other protocols. Firstly, the duty cycle adaptation mechanism enables the receiver node to wake up frequently depending on the traffic to facilitate receiving packets at a higher rate. Secondly, the request-based approach enables the sender to send packets from its queue after a successful transmission for a single listen cycle of the receiver. Thus, the TR-MAC protocol with a combination of duty cycle based 
adaptation and request-based approach can successfully deliver the offered load faster with very few queue overflows at the sender node. We also observe that the throughput with only the traffic-adaptive duty cycle adaptation approach is little lower than the combined approach, but still better than the request-based burst approach of the WiseMAC protocol. The throughput of the WiseMAC protocol is based on the request-based approach using More Bit, which gets limited to the burst length or the queue size at the sender node. The WiseMAC protocol does not add extra listen cycles to accommodate higher traffic, rather it continues to operate with the base check interval. Hence, the performance of WiseMAC protocol gets limited for a relatively higher traffic due to the lack of the duty cycle adaptation mechanism. The adaptation mechanism of the X-MAC protocol deals with adjusting the check interval based on the traffic-arrival, but it does not employ any traffic or request-based adaptation mechanism like the two other protocols. As the check interval was fixed for this experiment, thus the $\mathrm{X}$-MAC protocol experiences a lower throughput, and higher queue overflow for higher traffic.

\subsubsection{Delay}

The average delay per received packet is represented in Figure 4.4 using logarithmic scale and $95 \%$ confidence interval for varying traffic load for a pair of nodes. The base check interval was fixed to 1 second, and the packet arrival rate was increased at the sender node. Similar to the previous experiment, the TR-MAC protocol uses state increase parameter $(K)$ set to 5 , and state decrease parameter $(L)$ set to 10 . In this figure, we observe that the TR-MAC protocol with DCA and More Bit experiences significantly lower per packet delay for increasing traffic. This is achieved because of the inherent mechanism of the protocol combining the receiver-driven traffic-adaptive duty cycle adaptation approach, and the sender-driven request-based approach, as explained in Section 4.3. The TRMAC protocol with the combined approach can wake up as many as 27 times with the duty cycle adaptation algorithm at its highest state, and can deliver packets as many as the queue-size using the request-based More Bit mechanism. Comparatively, the delay per packet with only the traffic-adaptive approach 


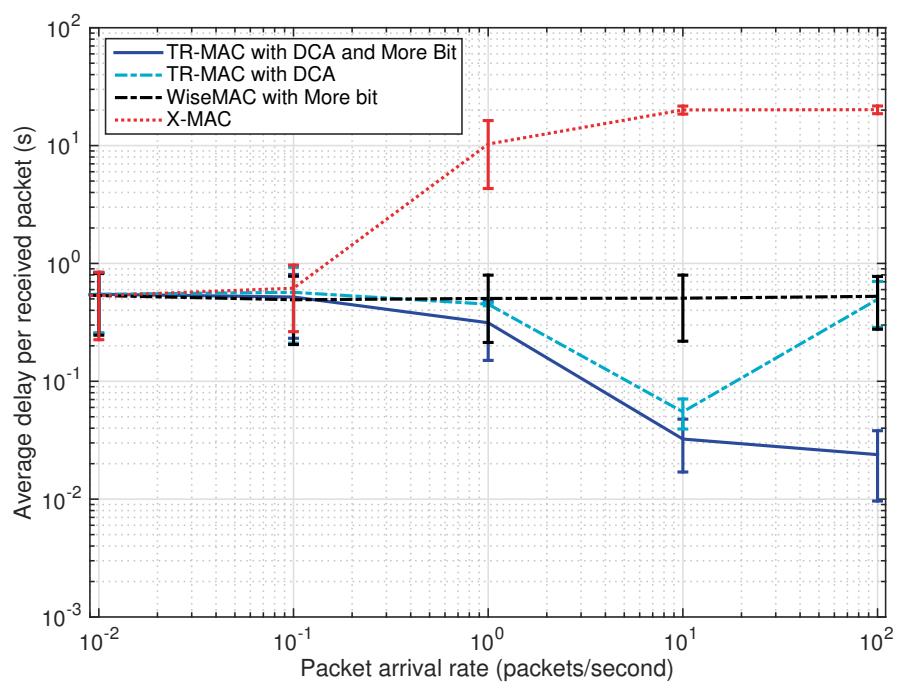

Figure 4.4: Average delay per received packet with varying traffic

provides better result at a traffic rate of 10 packets per second, but cannot keep up with the combined approach at a traffic rate of 100 packets/second. The delay per packet remains constant for the WiseMAC protocol at a higher traffic rate, because it only uses the sender-driven request-based More Bit approach to deliver all the packets in the queue whenever the sender gets access to a listen cycle of the receiver. The WiseMAC protocol cannot achieve the per packet delay like the TR-MAC protocol, since it uses the listen cycles using the fixed check interval, and does not add extra duty cycles based on the increasing traffic rate. On the other hand, the X-MAC protocol experiences higher per packet delay for higher traffic as the check interval was fixed for this experiment, and this protocol does not use a traffic adaptation mechanism like the other two protocols. 


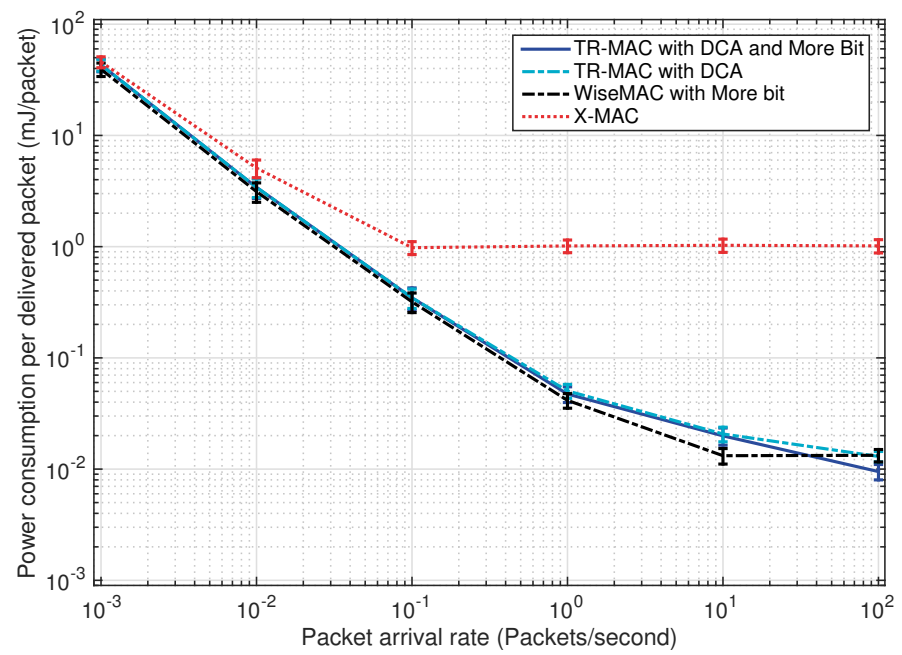

Figure 4.5: Energy-efficiency with varying traffic

\subsubsection{Energy-efficiency}

The simulation scenario to analyze the power consumption per delivered packet is similar to the previous simulation scenarios. The parameters for packet duration and transmission powers for these protocols are chosen as the system parameters given in Chapter 3. The check interval is fixed to 1 second, and the protocols employ traffic-adaptive mechanisms based on the increasing traffic rate. To compute the power consumption per delivered packet, the total power consumption during the simulation is divided by the number of successfully delivered packets. When a protocol can deliver higher number of packets, then it consumes higher amount of power. However, when the total power consumption is divided by a higher number of successfully delivered packets, then the power consumption per delivered packet gets smaller. Therefore, in this figure we observe that the power consumption per delivered packet decreases with increasing number of packet arrival rates, which leads to increasing number of successfully delivered packets by using the traffic-adaptation algorithm. 
The power consumption per delivered packet is presented in Figure 4.4 for varying traffic load with $95 \%$ confidence interval. We observe that the energy per delivered packet for the TR-MAC protocol with DCA and with More Bit is quite close to the energy consumption per delivered packet of the WiseMAC protocol. Recall from the system parameters for the transmission power of these protocols given in Table 3.2 that the TR-MAC protocol uses twice power compared to the WiseMAC and the X-MAC protocol, because of its underlying transmitted-reference modulation. Thus, eventually the TR-MAC protocol would provide better energy consumption, if the same transmission power were used for transmitting both the data packet and the acknowledgement packet. Furthermore, we have seen from the throughput results that the TR-MAC protocol with its combined traffic-adaptive approach is capable of delivering much higher packets compared to the WiseMAC protocol for higher amount of packet arrival rate. Thus, the TR-MAC protocol consumes higher amount of power per delivered packet, since higher number of packet transmissions accounts for higher energy consumption. Therefore, the energy-efficiency for the TR-MAC protocol with traffic-adaptive duty cycle adaptation combined with the request-based burst packet transfer approach in the presence of event-driven higher traffic can be considered satisfactory compared to the other protocols.

The X-MAC protocol operates in the unsynchronized link state by sending packets and waiting for the acknowledgements, and continuously performs this operation when it has more packets to send in its queue. This X-MAC protocol does not use any synchronized link state to minimize the preamble duration from the basic of maximum of the check interval duration by remembering the receiver's next wake up time, hence consumes higher amount of power for successfully delivering a single packet. Also the total number of delivered packet is limited for the X-MAC protocol, as we have seen in the earlier results. As a result, the energy per delivered packet for the X-MAC protocol stalls much earlier than the rest of the protocols, since dividing the total power consumption by the lower number of delivered packets yields to higher power consumption. 


\subsubsection{Parameter analysis}

In this section, we investigate the optimum state increase and decrease parameters for our proposed traffic-adaptive duty cycle adaptation algorithm for varying traffic loads. We analyze different combinations of system state increase parameter, $K$, and state decrease parameter, $L$, to find out the optimum combination ensuring both adaptability and stability. Recall that, the state increase parameter, $K$, determines whether one node will move to a higher state by adding extra listen cycles based on consecutive packet reception in its listen cycles. Correspondingly, the state decrease parameter, $L$, determines whether one node will move to a lower state by removing the previously adding extra listen cycles based on consecutive empty listening in those extra listen cycles. One node needs to adapt quickly to move to a higher state by decreasing the check interval after realizing an increase of traffic towards it. After moving to a new state, the node needs to operate in a stabilized manner by not moving back and forth to upper or lower states. Stability can be achieved by setting the parameters to a higher value, which in turn provides lower adaptability. Therefore, a tradeoff between adaptability and stability exists to decide for a combination of optimum state increase and decrease parameter. To analyze this tradeoff, we evaluate the traffic rate variation from three perspectives: the traffic increasing part, the stable traffic part, and the traffic decreasing part. We have conducted an experiment using 1 second base check interval where initially we offer 0.5 packets per second of traffic for first 50 seconds, then increase the traffic to 10 packets per second and maintain this rate for 1000 seconds, afterwards the traffic is decreased to initial 0.5 packets per second.

Firstly, we analyze the adaptability for fast state changing during traffic load increase by measuring the maximum delay at the transition point. Figure 4.6 represents distributions in form of box-plots for maximum packet delay experienced for different state increase parameters, $K$, over 100 simulations. On each box in a box-plot, the central mark indicates the median of all the data points, and the bottom and top edges of the box indicate the 25th and 75th percentiles, respectively. The 25th percentile is also known as the first quartile, the 50 th percentile as the median or second quartile, and the 75 th percentile as 


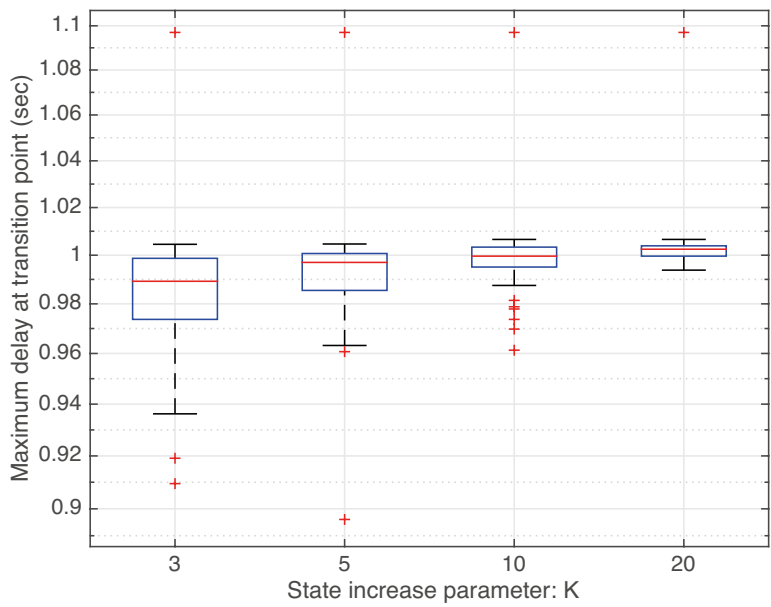

Figure 4.6: Maximum delay during traffic load increase

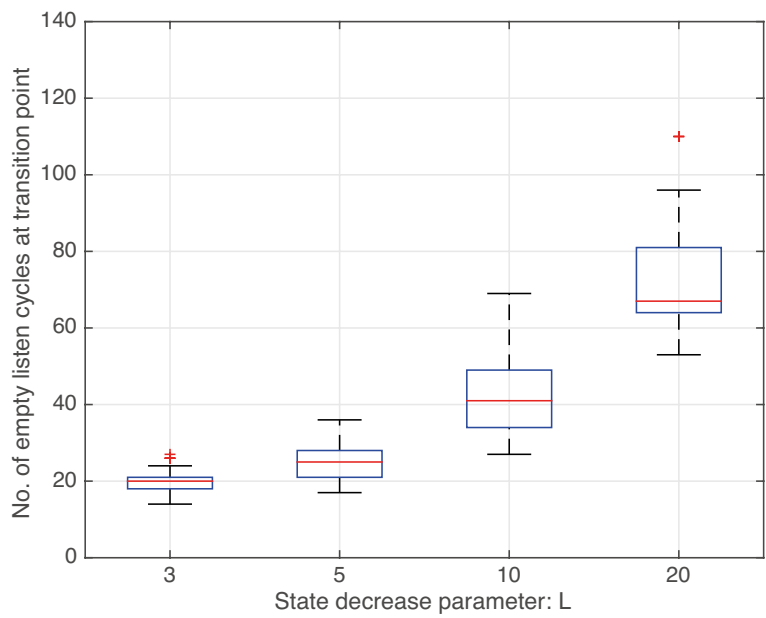

Figure 4.7: Number of empty listening during traffic load decrease 
the third quartile among the spread of all the data points. Thus, all the data points are essentially divided into four groups. The lines in both sides outside each box are called whiskers. These whiskers extend to the most extreme data points, which are not considered outliers. The outliers are plotted individually using the ' + ' symbol. Here we see that the maximum delay at transition point is close to the used check interval value of $1 \mathrm{~s}$, because of the request-based burst transfer part of the combined traffic-adaptive algorithm. In the figure, we observe that a smaller state increase parameter value provides relatively smaller delay to move to the next state, and eventually offers quick adaptivity. However, we do not see a significant difference in delay among the parameter values, since the initial traffic is increased to a significantly higher traffic rate. Thus, the node could move quickly to a higher state after receiving consecutive packets in $K$ number of successive listen cycles.

Secondly, we analyze the effect of adaptability during traffic load decrease for different state decrease parameters, $L$. If one node takes too long to decrease to a lower working state, then it consumes extra energy because of empty listenings allocated earlier. Figure 4.7 represents the number of empty listenings that occur for 20 seconds after the traffic rate is decreased for different state decrease parameters, $L$. We see that a higher state decrease parameter value implies higher number of empty listen cycles before moving decrease to a lower state. Thus, a lower state decrease parameter provides higher energy saving and higher adaptability. However, higher adaptability is achieved at an expense of lower stability, since one node can move to a lower state after missing only few packets consecutively.

Finally, we analyze the stability of the algorithm for a long stable traffic scenario from both delay and energy consumption perspectives. Figure 4.8 represents average delay per packet, and Figure 4.9 represents the energy per delivered packet for various asymmetric combinations of state increase parameter, $K$ and state decrease parameter, $L$ for stable traffic scenario. Here we observe that the combination of a lower value for the state increase parameter, $K$, and a higher value for the state decrease parameter, $L$, provides lower delay per packet. Hence, this combination enables one node to move quickly to a higher 


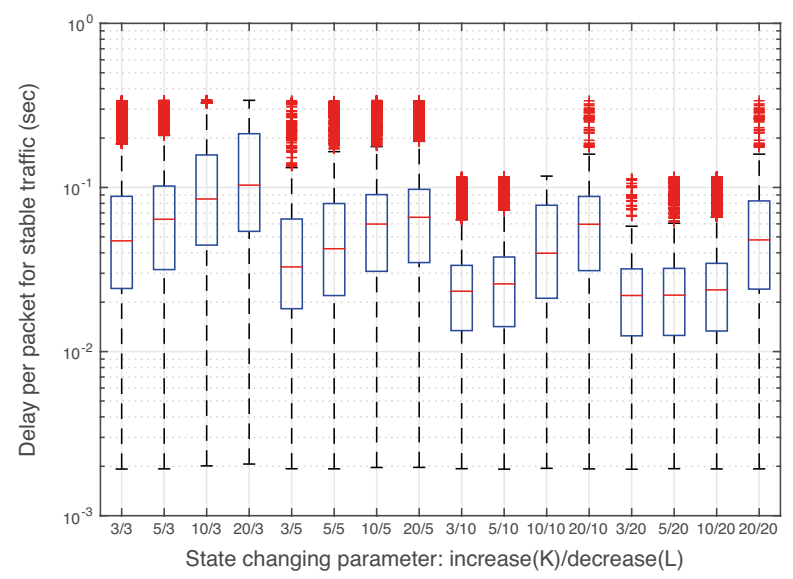

Figure 4.8: Delay per packet for stable traffic

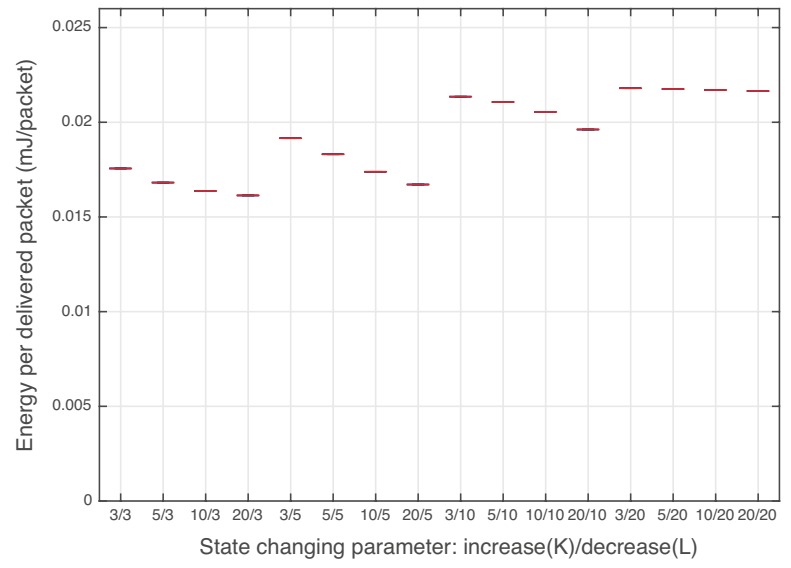

Figure 4.9: Energy per packet for stable traffic

state because of the lower value of the state increase parameter, $K$, and remain in the higher operating state for a longer duration because of the higher value of the state decrease parameter, $L$. As a result, this combination provides quick 
adaptability, and higher stability in an operating state of the traffic-adaptive duty cycle adaptation algorithm. However, one node cannot quickly move down to a lower operating state with this combination. Hence, this combinations has relatively higher power consumption because of the empty extra listen cycles of the node in a higher operating state.

Alternatively, the combination of a higher value for the state increase parameter, $K$, and a lower value for the state decrease parameter, $L$, provides higher delay per packet. As a result, this combination has slow adaptability to move to a higher operating state because of the higher value of the state increase parameter, $K$. On the other hand, this combination provides fast adaptability to move to a lower state. Hence, it compromises with the stability, because of the short operating time in a higher operating state. This combination consumes lower amount of energy because of the fast adaptability to move to a lower state causes very few empty listen cycles in a higher states. However, the difference of the extra energy consumption of this combination with its opposite combination is not significant.

Considering all these factors into account, we propose to choose a combination of smaller state increase parameter $K$ and higher state decrease parameter $L$ to minimize the delay per packet, to provide quick adaptability to move to higher state in the presence of higher event-driven traffic, and to provide relatively higher stability in an operating state, for example, $K=5$ and $L=10$. Given this setting, we could trade delay for energy consumption, and adaptability for stability by optimally tuning the state changing parameters.

\subsubsection{Performance with packet loss}

In this section, we analyze the impact of packet loss on the traffic-adaptive duty cycle adaptation algorithm together with the request-based burst packet transfer, as discussed in Subsection 4.3.3. Recall that the traffic-adaptive duty cycle adaptation algorithm triggers one node to move to a higher state with higher transmissions after receiving consecutive packets in $K$ number of consecutive listen cycles, and triggers to move down to a lower state with lower transmissions after missing consecutive packets for $L$ number of those extra 


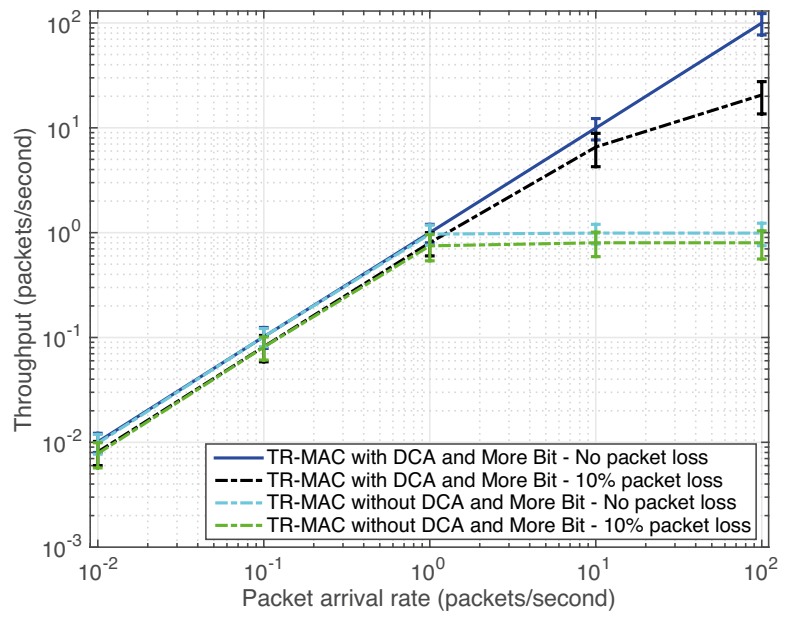

Figure 4.10: Throughput with varying traffic with packet loss

added listen cycles. Furthermore, the consequence of missing a packet in the middle of a burst for the request-based transfer algorithm stops the burst right at that moment. We simulate similar simulation scenarios for the traffic-adaptive algorithm combined with the request-based burst packet transfer in the presence of $10 \%$ packet loss, where the nodes start with the base check interval of 1 second. It means that, around $10 \%$ of the packets are randomly chosen as lost, which includes both the data packet and the acknowledgement packet. In this section, we present an analysis of the throughput, delay, and energy-efficiency in the presence of $10 \%$ packet loss.

\subsubsection{Throughput}

The simulation scenario to analyze the throughput is similar to the previous simulation scenarios. The TR-MAC protocol starts with the base check interval of 1 second, and increases the number of transmissions by moving to a higher state triggered by the traffic-adaptive algorithm for increasing traffic rate. The throughput is calculated by dividing the total number of delivered packets by 
the total simulation time. The throughput results with $10 \%$ packet loss with and without the traffic adaptive algorithm are presented in Figure 4.10. Here we see that the throughput with the traffic adaptation algorithm with DCA and with More Bit for the TR-MAC protocol with 10\% packet loss is lower than the throughput without any packet loss. This happens because one receiver node cannot move to a higher state in the case of a data packet loss. This movement to a higher state requires consecutive packet reception without any interruption for $K$ listen cycles. Recall that $K$ represents the state increase parameter. Missing a data packet breaks this consecutive packet reception for the listen cycles. As a result, the throughput does not increase as much as the case without any packet loss for higher packet arrival rate. The throughput increase also gets affected for the case of an acknowledgement packet loss, as the sender node does not send packets at a higher rate although the receiver node may operate at a higher rate after triggering to move to a higher state by receiving consecutive packets in $K$ listen cycles. Furthermore, the throughput is affected after a packet loss within the burst. In this case, the burst packet transfer cannot continue with the burst, as the lost packet has to be retransmitted. Moreover, the loss of the first packet in the burst resets the state increase parameter $K$ to zero. As a result, one node needs to receive consecutive packets again for $K$ listen cycles to move to a higher state. Hence, we achieve lower throughput with the traffic adaptation algorithm for packet loss.

Figure 4.10 also presents the throughput without the traffic-adaptive algorithm with and without packet loss. Without the traffic adaptive algorithm, we see that the throughput does not increase when the packet arrival rate becomes higher than the check interval duration. In this case, one pair of node operates with the base check interval of 1s. Hence, they cannot increase the amount of transmissions with respect to the increasing packet arrival rate. We also observe that the throughput with $10 \%$ packet loss becomes further lower than the throughput without packet loss case. 


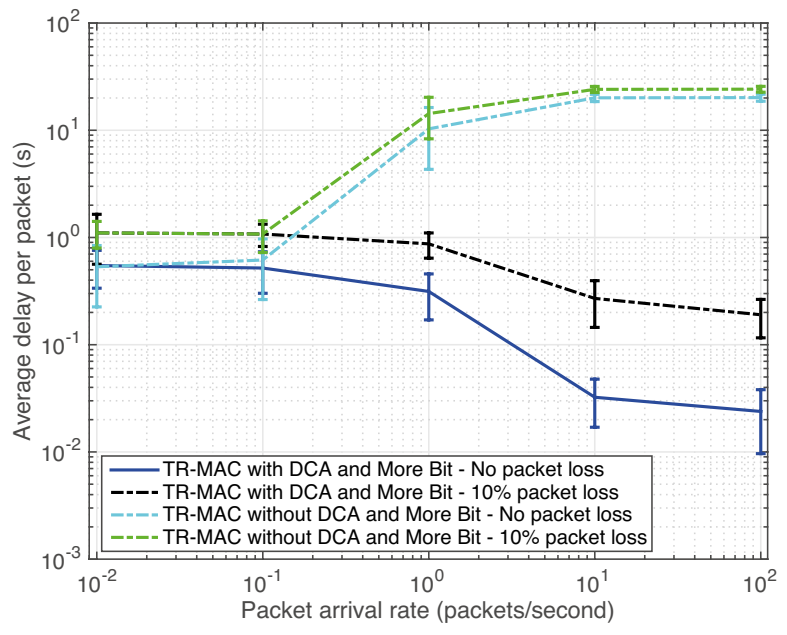

Figure 4.11: Average delay per packet with packet loss

\subsubsection{Delay}

We present the delay per packet results with no packet loss and $10 \%$ packet loss with and without the traffic-adaptive algorithm in Figure 4.11. Here we observe that the delay per packet with the traffic adaptive algorithm together with the burst packet transfer for $10 \%$ packet loss scenario is higher than the delay per packet without any packet loss scenario. The reason is that the receiver node fails to trigger to move to a higher state if it looses a data packet, because a data packet loss event resets the value of the state increase parameter $K$ to zero. As a result, the pair of the nodes are forced to communicate in the lower state, as they fail to move to a higher state based on the increasing packet arrival rate. From the moment of a packet loss, the receiver node again needs to receive consecutive packets without any packet loss for consecutive $K$ number of listen cycles to move to a higher state. The effect of an acknowledgement packet loss is higher than the data packet loss. The reason is that, the receiver node moves to a higher state by receiving consecutive data packets in $K$ listen cycles, while the sender fails to move to a higher state because of missing an acknowledgement 


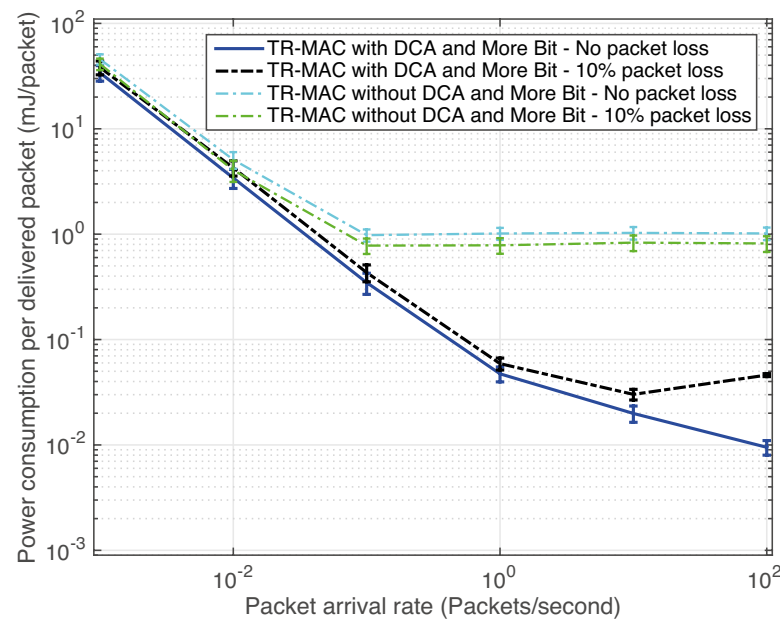

Figure 4.12: Energy-efficiency with varying traffic with packet loss

packet in the consecutive packet reception process. In this scenario, the sender node does not send at a higher rate, even though the receiver node already scheduled few extra listen cycles. Thus, the delay per packet increases in the presence of data or acknowledgement packet loss.

Furthermore, the request-based burst packet transfer fails to send all the packets from its queue in the presence of packet loss, because the burst stops as soon as one data packet within the burst is lost. As a result, all the packets in the queue experience higher delay, because all of them needs to wait to be delivered during the next listen cycle of the receiver node. Thus, a packet loss within a burst also has an impact on the delay per packet parameter. Note that, loosing the first packet of the burst marks a packet miss in the state increase parameter $K$ of the duty cycle adaptation algorithm, while loosing any other packet of the burst does not have any impact on that parameter. 


\subsubsection{Energy consumption}

The simulation scenario to analyze the power consumption per delivered packet is similar to the previous simulation scenarios. The traffic-adaptive algorithm is applied by the TR-MAC protocol based on the increasing traffic rate. The total power consumption during the simulation is divided by the number of successfully delivered packets to compute the power consumption per delivered packet. The number of successfully delivered packets increases by using the traffic-adaptation algorithm, which consumes higher amount of power. It means that the protocol have delivered higher number of packets. However, the power consumption per delivered packet becomes smaller when the total power consumption is divided by a much higher number of successfully delivered packets. As a result, we observe in the figure that the power consumption per delivered packet decreases with increasing number of successful packet deliveries.

The power consumption per delivered packet is presented in Figure 4.12 for varying traffic load with and without packet loss. We observe that the energy per delivered packet for the TR-MAC protocol with DCA and with More Bit without any packet loss consumes lower power per successfully delivered packet than the case with $10 \%$ packet loss. The pair of nodes needs to receive consecutive packets in $K$ listen cycles to move to a higher state with higher number of transmissions, which does not happen in the case of packet loss. Rather, the parameter $K$ needs to start recounting the number of consecutive packet receptions once a packet has been lost. Loss of acknowledgement packets interrupts the consecutive acknowledgement packet reception, thus restricts the movement to a higher state for the sender node. As a result, the sender node cannot send packets at a higher rate, although the receiver node may be operating at a higher state by receiving consecutive data packets. Furthermore, the packet loss within a burst stops the burst immediately. We also observe in the figure that power consumption per delivered packet without any traffic adaptation algorithm is much higher than with traffic adaptation algorithm. The reason is, relatively lower number of packets gets delivered without the traffic adaptation algorithm. As a result, the power consumption per delivered 
packet becomes higher. With the $10 \%$ packet loss, the power consumption per delivered packet without the traffic adaptation algorithm becomes even higher, as the number of successfully delivered packets decreases with the packet loss.

\subsection{Summary}

In this chapter, we present a traffic-adaptive duty cycle adaptation algorithm combined with request-based burst packet transfer for the TR-MAC preamble sampling protocol for wireless sensor networks. The traffic-adaptive duty cycle adaptation algorithm increases the duty cycles of the receiver node based on the incoming traffic, and the corresponding sender node can follow the duty cycle of the receiver node without extra information exchange. As a result, it is useful to quickly adapt to sudden variation of traffic generated by event-driven scenarios. On the other hand, the request-based burst transfer mechanism maximizes the number of packet deliveries between a pair of nodes using a single bit overhead. We present an analysis about the stability and adaptability of our algorithm to provide an in depth knowledge of the algorithm. In addition to that, we present the consequence of packet loss for this traffic-adaptive duty cycle adaptation algorithm combined with request-based burst packet transfer. Furthermore, we evaluate our proposal in comparison with other protocols, and show that our proposed solution achieves better results for quality of service parameters, for example, throughput and per packet delay. The traffic-adaptive duty cycle adaptation combined with request-based burst packet transfer presented in this chapter provides adaptability for traffic varying scenarios in WSNs by maximizing the throughput, minimizing delay per packet, and at the same time maintaining energy-efficiency with very limited explicit information exchange within the WSN. 



\section{Modelling multi-channel multiple access using frequency offsets}

A system using frequency offset based transmit reference modulation allows multiple nodes to transmit simultaneously and asynchronously using multiple frequency offsets without any mutual timing coordination. Thus, such a system provides inherent capabilities for multiple access in the MAC layer to coordinate the shared use of the common wireless medium among the nodes of the wireless sensor network. However, certain characteristics of a frequency offset based system limits its performance as discussed in Section 2 of Chapter 2. For example, the number of available frequency offsets is limited being dependent on several system parameters, and the number of simultaneous communications using different frequency offsets is limited due to inter-user interference. In order to obtain an understanding of the consequences of these limiting factors, and the throughput and efficiency potential at the MAC layer, we model the effect of frequency offset based TR modulation on a basic multiple access scheme. In this chapter, we introduce an extended version of the performance model of a basic multi-channel slotted-Aloha system, which captures the basic phenomena of a multi-channel system with a limited set of channels and a limit to the number of simultaneously used channels. An analysis of this model reveals the potential of a multiple access control in a MAC protocol for TR modulation with frequency offsets.

This chapter answers research question 4 ("How do the multi-channel properties affect the performance of multiple access control in a system using transmit 
reference modulation with frequency offsets?"). In this chapter, we propose and analyze a model for a multiple access scheme using frequency offsets, which is based on our published paper [23]. As our starting point, we take a basic model of single channel slotted Aloha (S-Aloha) [73]. In S-Aloha, a channel is divided into several slots, where transmissions always start at the beginning of a slot. S-Aloha does not perform carrier sense to check whether the channel is busy or not. A successful communication in S-Aloha requires only one packet is transmission during a slot duration. We extend the single channel S-Aloha model for multi-channel S-Aloha with similar principles representing the possibilities of multiple simultaneous transmissions using frequency offsets. In the multi-channel S-Aloha model, a node can randomly and independently choose any of the channels for a time slot to transmit a packet. A transmission is successful if the chosen channel was unique for that time slot; i.e., no other node was transmitting in the same channel (with the same offset) during that slot. Finally, we extend this model further to take into account the specific limitations for frequency offset based TR modulation that there is a limit to the number of simultaneously used channels (offsets). The result is a model that describes the behaviour of a slotted, Aloha-based access mechanism for WSNs based on transmit reference modulation with multiple frequency offsets. This model provides fundamental insights into what limits the performance of a MAC protocol for frequency offset based TR modulation.

The contributions of this chapter are: i) we present a general model for a multichannel slotted Aloha-based multiple access scheme where transmissions are successful if a unique channel is chosen, and if the total number of transmissions is below a certain limit; ii) we use the model to provide fundamental insight into the performance of a MAC protocol for transmit reference modulation with frequency offsets; iii) as an intermediate product, we present a model for straight multi-channel slotted Aloha; and iv) we validate our models by comparing to results from a simulation model.

The remainder of this chapter is organized in the following manner: Section 5.1 presents the motivation for this work. Afterwards, Section 5.2 presents the related work to this research. Section 5.3 provides the multiple access modelling 
scheme that starts with S-Aloha, followed by an extended model for multichannel S-Aloha. Finally, this section concludes with a model to represent frequency offset based TR modulation. Section 5.4 presents the results and analysis in terms of efficiency and throughput of the system. Finally, Section 5.5 provides the summary of our work.

\subsection{Motivation}

Wireless sensor networks mostly use single channel systems for operation. However, throughput and capacity performance of single channel systems decline as the number of communicating nodes increases with the prevalence of IoT. Thus, WSNs need to have an efficient multiple access scheme with multiple channels. A multi-channel system attempts to increase the system throughput and efficiency by using multiple frequency channels. However, this performance improvement comes at the cost of increased power consumption, channel switching delay, channel coordination between sender and receiver, and adjacent channel interference [26]. The power consumption of a node increases if multiple transceivers are used to realize multi-channel communication. A single tunable transceiver to operate on different frequencies adds the overhead of channel switching delay. Most importantly, a detailed coordination for channel switching is necessary among the nodes to ensure that the receiver is on the same channel with the sender at the same time to receive the packet. However, there exist single channel systems that could offer performance like a multichannel system with limited complexity. TR modulation is one of such single channel systems.

Transmit reference modulation offers multiple frequency offsets, which can be used as virtual frequency channels for multiple access [17]. The sender using TR modulation modulates a reference signal with data, and sends both the modulated signal and the reference signal in the shared medium with a frequency offset known to the receiver. The receiver restores the transmitted data by correlating the received signal with a version of itself having the same frequency offset applied. In a TR system, the receiver only needs to know the frequency 
offset used by the sender in order to decode the received signal [19]. Thus, TR modulation offers a simplified solution to the channel coordination problem if nodes work in pairs. Moreover, multiple nodes can transmit simultaneously by employing several frequency offsets without the need for mutual timing coordination. This performance improvement comes without using multiple transmitters and channels switching delays.

The frequency offset based transmit reference modulation system provides several opportunities that can be utilized in the upper layers. Exploiting this TR modulation in the physical layer, earlier the authors proposed a MAC layer protocol in [19, 20,21,22], called TR-MAC for low duty cycle and low data rate operation. The nodes using TR-MAC operate in pairs in synchronized link states by remembering the frequency offset to be used for future communication. As a preamble sampling protocol, the nodes using TR-MAC protocol perform independent duty cycling, and sleep most of the time to save energy. Furthermore, this duty cycling ensures that only few nodes communicate at a time, thus only few frequency offsets are used simultaneously. As a result, a single channel can be used for multiple access communication by different pairs of nodes using different frequency offsets. The communication is successful if different pairs of nodes within the range choose mutually exclusive frequency offsets. Multiple pairs of nodes choosing the same frequency offset within the range of a receiver would result in a collision, since the intended receiver would not be able to decode the transmitted signal properly. Thus, TR-MAC protocol exploits the frequency offset based TR modulation in the physical layer to provide more flexibility towards the upper layers.

Although frequency offset based TR modulation has its advantages, there are also some parameters that limit its performance. In a network with multiple access using frequency offset based TR modulation, there can be a number of simultaneous communication links using different frequency offsets. If a frequency offset is larger than the channel coherence bandwidth, it causes the reference and information signals to loose coherence between them. As a result, the despreading suffers from frequency-selectivity of the propagation channel, leading to a weak despread signal. Hence, there exists a limitation on the 
number of available frequency offsets [17]. Moreover, the frequency offset based TR modulation has an impact in the receiver side due to the overall increase of noise-floor causing multi-user interference in the presence of a large number of active links with multiple frequency offsets. The noise is further enlarged by the self-mixing of the reference and information signals, thus restricting the number of active interfering links using frequency offsets in the network [17]. Therefore, we can conclude that the frequency offset based multiple access has two characteristics that limit its performance: i) The number of available frequency offsets gets limited, and depends on the desired data rate, symbol rate of the used modulation, and delay spread of the environment; and ii) The number of simultaneous communications using different frequency offsets becomes limited, since the receiver experiences problems to decode the signal within the increased interference level in the presence of several signals using different frequency offsets. Therefore, a suitable model for frequency offset based multiple access technique is needed that takes care of these limitations for coordinating the shared medium access in WSNs.

\subsection{Related work}

In wireless communication, medium access control has been analyzed for several decades. Various multiple access protocols are available in the literature $[74,75]$. Aloha was the first multiple access protocol that allows a node to transmit a packet as soon as it receives the packet from the upper layers [76]. In case of a collision, the senders retransmit the packet with a certain probability $p$ after waiting a random amount of time. With this simple mechanism, Aloha provides a maximum of $18 \%$ channel efficiency under the assumption of a large (infinite) number of transmitters with Poisson arrivals. Afterwards, S-Aloha was proposed where a channel is divided in several time slots, and nodes defer the packet transmission to the start of a time slot [73]. The maximum efficiency of S-Aloha is $37 \%$ after using the time slot based mechanism. S-Aloha may be unstable in several scenarios as mentioned in [77].

A generic multi-channel system consists of several independent channels. To 
transmit a packet, a user can choose any of these channels using a suitable multiple access protocol. A simple multi-channel system could be a multichannel slotted-Aloha system that consists of multiple equal-capacity slotted channels shared by several users, where a user first chooses a channel, then chooses a slot to transmit a packet. A multi-channel S-Aloha protocol has been studied in [78], and a stability optimization criterion with and without receiver collision has been studied in [79]. This multi-channel system improved performance, and offered cost reduction and fault tolerance compared to the single channel system. The performance of multi-channel slotted-Aloha system was also analyzed in [80] and [81] with a deterministic channel model without multi-path fading. The authors in [82] discussed about stabilizing the multichannel S-Aloha from the context of machine to machine (M2M) communication. While previous researches consider use of separate channels, using frequency offsets that belong to the same frequency channel has not been addressed before. In this chapter, we propose a multiple access model for a multi-channel system with frequency offsets using a multi-channel S-Aloha model.

\subsection{Modelling multiple access schemes}

The multiple access problem deals with the coordination of multiple sending and receiving nodes sharing a common medium. In this section, we start with the basic multiple access scheme of S-Aloha for a single channel. Afterwards, we model a simple multiple access scheme for multiple channels using S-Aloha. Finally, we model the multiple access technique with multiple frequency offsets.

\subsubsection{S-Aloha system}

S-Aloha is a simple multiple access technique for a single channel, where the channel is divided into time slots. A node always transmits in the beginning of a time slot. Whenever a node receives a packet to transmit, the transmission is delayed until the start of the next time slot [76]. A transmission can be successful if only one packet is transmitted during a slot. Collision happens 
in the presence of multiple packets in a single time slot. In case of a collision, a node will retransmit the packet in each next slot with probability $p$ until the packet is transmitted successfully.

In modelling S-Aloha, it is usually assumed that the probability $p$ that a certain node transmits within a time slot for a fresh packet is the same as for a retransmitted packet that suffered a collision. Hence, the average number of packet transmissions in the system within a time slot can be given by $\lambda=n p$, where $n$ is the number of active nodes. Let us now define the random variable $K$ as the number of nodes that are transmitting simultaneously. If we assume the packet arrival process is a Poisson process, the probability of $k$ arrivals, or equivalently $k$ transmission attempts, within a unit time is given by [83] as

$$
P(K=k)=e^{-\lambda} \frac{\lambda^{k}}{k !}, k=0,1,2,3 \ldots
$$

Therefore, the average number of packets that can be successfully delivered, that is, the throughput, $S_{S}$, for single channel S-Aloha system is given by [77] as

$$
\begin{aligned}
S_{S} & =\mathbb{E}(\text { No.of successfultransmissions per slot }) \\
& =P(K=1)=\lambda e^{-\lambda} .
\end{aligned}
$$

S-Aloha only provides successful transmissions without collision when only one transmission attempt is made per time slot. The efficiency of the S-Aloha system would be the same as its throughput because of the availability of only one channel, and can be given by

$$
E_{S}=\lambda e^{-\lambda}
$$

\subsubsection{Multi-channel S-Aloha system}

In this section, we extend the model of S-Aloha for multiple channels. The working principle of multi-channel S-Aloha would be similar to that of S-Aloha with $C$ narrowband S-Aloha channels in parallel occupying the same bandwidth as the S-Aloha system. This way, the collision domain would be limited to that 
particular channel. A node would select a channel randomly and independent of other nodes to initiate a packet transmission. The communication would be successful if one node chooses a unique channel for a single time slot. Collision happens if multiple transmitters choose the same channel for a single time slot.

Therefore, the system throughput, $S_{M}$, for a multi-channel S-Aloha system with $C$ channels can be derived as

$$
\begin{aligned}
S_{M}= & \mathbb{E}(\text { No. of successful transmissions per timeslot }) \\
= & \sum_{k=1}^{\infty} P(K=k) \\
& \times \mathbb{E}(\text { No. of successful transmissions per timeslot } \mid K=k) \\
= & \sum_{k=1}^{\infty} P(K=k) \\
& \times \sum_{s=1}^{k} s P(\text { s out of } k \text { choose unique channels out of } C \text { channels }) \\
= & \sum_{k=1}^{\infty} e^{-\lambda} \frac{\lambda^{k}}{k !} \cdot \sum_{s=1}^{\min (k, C)} s P(C, k, s),
\end{aligned}
$$

where $P(C, k, s)$ denotes the probability of $s$ successful transmissions when $k$ nodes are transmitting simultaneously, and $C$ channels are available. In Eq. 5.4, we have also used the fact that the number of successful transmissions $(s)$ cannot be more than the number of attempts $(k)$ or the number of channels $(C)$ present. That is why, $\min (k, C)$ is used in the expression. An expression for $P(C, k, s)$ is given later in this section.

The efficiency of the multi-channel S-Aloha system can be derived by normalizing the throughput by the number of available channels, $C$, and can be given by

$$
\mathbb{E}_{M}=\frac{1}{C} \sum_{k=1}^{\infty} e^{-\lambda} \frac{\lambda^{k}}{k !} \cdot \sum_{s=1}^{\min (k, C)} s P(C, k, s) .
$$




\subsubsection{Multiple access scheme using frequency offsets}

In this section, we present a multiple access model using multiple frequency offsets. In a system with transmit reference modulation, the multiple access scheme can be based on multiple frequency offsets with different nodes using different frequency offsets to communicate with each other. To transmit a packet, one node using transmit reference modulation can randomly choose a frequency offset from the frequency offset pool, and modulate the carrier signal with the chosen frequency offset. The receiver can use the same frequency offset to decode the received signal in order to retrieve the original transmitted data. Therefore, the frequency offset pool can be referred to as a collection of virtual channels.

In a frequency offset based multiple access system, a node could independently and randomly choose a frequency offset to initiate a packet transmission. A transmission is successful only if the frequency offset chosen by one node is not chosen by any other node within its range at the same time. Collision occurs if another node within the coverage area chooses the same frequency offset to transmit simultaneously. As a result, a multiple access scheme using frequency offsets can be modelled using similar principles as multi-channel S-Aloha. The parameter $C$ now corresponds to the number of maximum available frequency offsets for multiple access with frequency offset, instead of number of available channels.

Besides the similarities between the multi-channel S-Aloha and the frequency offset based multiple access, there are two important differences between these systems. Firstly, the number of unique frequency offsets in the offset pool is variable, and depends on the system parameters, for example, data rate, coherence bandwidth, and delay spread of the environment. As a result, the total number of unique frequency offsets available for use gets limited. A calculation of the number of frequency offsets based on the system parameters is given in Subsection 5.4.1.

The second important difference between the multi-channel S-Aloha system and the frequency offset based multiple access system is that the number of simultaneous communications is limited with a maximum value of $m$ in frequency 
offset based multiple access model, due to the limit in acceptable interference level. Assuming a scenario where one receiver node can sense multiple transmissions using several different frequency offsets destined to different receivers. The total interference level for this receiver is increased by these simultaneous transmissions because of the cross-mixing among signals from different active nodes using different frequency offsets. The authors in [17] analyzed the limitation on simultaneous communications for a desired receiver in the presence of several simultaneous transmissions, and concluded at most 3 to 4 active transmissions within the acceptable interference level. It may even be possible to have fewer parallel transmissions towards the same receiver, which is out of scope of this chapter.

We can then derive the system throughput, $S_{F O}$, for a frequency offset based multiple access scheme with $C$ available frequency offsets, and maximum $m$ number of simultaneous communications as

$$
\begin{aligned}
S_{F O} & =\sum_{k=1}^{\infty} P(K=k) \mathbb{E}(\text { No. of successful transmissions } \mid K=k) \\
& =\sum_{k=1}^{m} P(K=k) \mathbb{E}(\text { No. of successful transmissions } \mid K=k),
\end{aligned}
$$

since with more than $m$ simultaneous communication attempts, none can be successful. This can be further simplified, similar to Eq. (5.4), as

$$
S_{F O}=\sum_{k=1}^{m} e^{-\lambda} \frac{\lambda^{k}}{k !} \cdot \sum_{s=1}^{\min (k, C)} s P(C, k, s)
$$

The efficiency of the frequency offset based multiple access system can be derived by normalizing the throughput by the number of maximum allowed simultaneous transmissions, $m$, and can be given by

$$
\mathbb{E}_{F O}=\frac{1}{m} \sum_{k=1}^{m} e^{-\lambda} \frac{\lambda^{k}}{k !} \cdot \sum_{s=1}^{\min (k, C)} s P(C, k, s) .
$$


The success probability, $P(C, k, s)$, is derived based on the condition where $s$ number of simultaneous communications become successful out of $k$ simultaneous transmission attempts by choosing unique frequency offsets out of $C$ frequency offsets. Recall that a node will be successful in transmitting only if the frequency offset chosen by the node is unique in the sense that it is not chosen by any other node. Since the total number of ways $k$ nodes can choose from $C$ frequency offsets is $C^{k}$, and each node chooses a frequency offset randomly and independently of each other, the success probability, $P(C, k, s)$, is given by

$$
P(C, k, s)=\frac{1}{C^{k}} f(C, k, s) .
$$

Here $f(C, k, s)$ provides the number of possible ways $k$ nodes can choose from $C$ frequency offsets with exactly $s$ nodes having selected a unique frequency offset. Note that exactly $s$ nodes select a unique frequency offset means that none of the remaining $(k-s)$ nodes select a unique frequency offset from the remaining $(C-s)$ frequency offsets. If $\left(\begin{array}{l}k \\ s\end{array}\right)$ represents the possible sets of nodes that can be successful, and ${ }^{C} P_{s}$ represents the possible ways this set of $s$ nodes can choose unique frequency offsets from $C$ frequency offsets, then we can write $f(C, k, s)$ as

$$
f(C, k, s)=\left(\begin{array}{l}
k \\
s
\end{array}\right){ }^{C} P_{s} f(C-s, k-s, 0) .
$$

Now we derive an expression for $f\left(C^{*}, k, 0\right)$ that represents the number of ways $k$ nodes can select frequency offsets from $C^{*}$ frequency offsets, and none having a unique frequency offset. This happens when any of the frequency offsets chosen by a node is also chosen by at least another node. This resembles to situations where the nodes form groups of size two or more, then choose a frequency offset as a group. For example, one possibility is when all of the nodes choose the same frequency offset, which is equivalent to all the nodes forming one group, then as a group choosing a frequency offset. Another possibility is that few of the nodes choose the same frequency offset as one group, and the rest of the nodes choose another frequency offset as another group. Since each group must contain at least two nodes, the maximum number of groups that can be formed is $\lfloor k / 2\rfloor$. 
We use 2-associated Stirling number of second kind [84, 85], $S_{2}(k, i)$, that represents the number of possible group formations from $k$ distinguishable objects into $i$ groups, where each group containing at least 2 objects. The number of ways these $i$ groups can choose from $C^{*}$ frequency offsets is represented by ${ }^{*} P_{i}$. Hence, the number of ways there can be no communication where there are $C^{*}$ frequency offsets and $k$ nodes, represented by $f\left(C^{*}, k, 0\right)$, can be expressed as

$$
f\left(C^{*}, k, 0\right)=\sum_{i=1}^{\lfloor k / 2\rfloor} S_{2}(k, i){ }^{C^{*}} P_{i} .
$$

The 2-associated Stirling number of second kind holds the following properties $[84,85]$ :

$$
S_{2}(k, i)= \begin{cases}0, & k<2 i \\ 1, & k \geqslant 2 \\ i \cdot S_{2}(k-1, i)+(k-1) \cdot S_{2}(k-2, i-1), & \text { other cases }\end{cases}
$$

\subsection{Results and analysis}

We have two goals in this section: i) we present the performance of frequency offset based multiple access model, and compare the analytical results with simulation performance, ii) we present the analytical results of multi-channel S-Aloha. Before presenting these results, we present a parameter analysis to calculate the number of available frequency offsets in the system. We implement the analytical models of the frequency offset based multiple access and the multichannel S-Aloha in Matlab. We also simulate the frequency offset based system using the discrete event simulator $\mathrm{OMNeT}++$. For the simulation, we consider a single server with many interfaces that can represent the multiple virtual channels using frequency offsets. One node randomly chooses a frequency offset, a virtual channel in this case, to communicate with the server. In the server, we impose the limitation on simultaneous communications imposed by the TR modulation using frequency offsets. The server concludes successful 
packet reception if different packets are received in different virtual channels for a single time slot within the permitted limitation on the number of simultaneous communications. In the rest of the section, we first present the parameter analysis. Afterwards, we present the results for both of these models in terms of the total throughput and efficiency by varying the offered load. For the frequency offset based multiple access model, we consider different limitations on the number of simultaneous transmissions. For statistical accuracy, 100 simulation runs are averaged, with 95\% confidence intervals. Afterwards, we present the analytical results for multi-channel S-Aloha model, which we developed as an intermediate step.

\subsubsection{Parameter analysis}

To analyze the performance of the frequency offset based multiple access model, it is important to know the parameter value of the maximum number of available frequency offsets in the system. In this section, we present a sample calculation to find out the number of available frequency offsets. Only a discrete set of frequency offsets are usable using frequency offset based transmit reference modulation system, as mentioned in Subsection 5.3.3. A frequency offset must be an integer multiple of symbol rate, $R_{b}$ [17]. The symbol rate is equivalent to the data rate for binary phase shift keying (BPSK) modulation. However, the physical layer imposes a restriction on the maximum number of usable frequencies to be used as a frequency offset, represented by $f_{\max }$. This maximum frequency offset has to be much smaller than the coherence bandwidth of the channel, $B_{C}$; that is, $f_{\max } \ll B_{C}$ to ensure the reference and the information signals are affected similarly by the channel [17]. As a rule of thumb, this condition translates as $f_{\max } \leqslant B_{C} / 10$. The coherence bandwidth is inversely proportional to the delay spread, $\tau_{r m s}$, which has typical value of $100 \cdot 10^{-9} \mathrm{sec}$ for an indoor office environment [86]. Therefore, the maximum frequency to be used as an offset, $f_{\text {max }}$, in an office environment is given by

$$
f_{\max } \leqslant \frac{B_{C}}{10}=\frac{\frac{1}{\tau_{r m s}}}{10}=\frac{\frac{1}{100 \cdot 10^{-9} s}}{10}=1000 \mathrm{KHz} .
$$


All the frequency offsets to be used should be less than the maximum frequency. For a data rate of $25 \mathrm{kbps}$, the corresponding symbol rate becomes $25 \mathrm{KHz}$. Thus, the number of available frequency offsets, $N_{F}^{A}$, is given by

$$
N_{F}^{A}=\frac{f_{\max }}{R_{b}}=\frac{1000 \mathrm{KHz}}{25 \mathrm{KHz}}=40
$$

However, the frequency offset in a particular link should not be twice the frequency offset of another simultaneous link, that is, $f_{i} \neq 2 f_{j}$ for $i \neq j$ [17]. Because after demodulation, the desired message signal of user 1 appears at baseband at $f_{1}=0$, as well as at an intermediate frequency band at $f=2 f_{1}$. It means that any user (say user 2), using a frequency offset of $f_{2}=2 f_{1}$, will suffer interference from user 1 at the demodulation stage. This condition is vitally important to minimize the inter-user interference and maximize the signal-to-noise ratio.

As a result, the number of usable frequency offsets, $N_{F}^{U}$, is given by [23] as

$$
N_{F}^{U}= \begin{cases}\frac{N_{F}^{A}}{2}+\sum_{d=1}^{d_{\max }} \Gamma\left(\frac{N_{F}^{A}+4^{d}}{2^{2 d+1}}\right), & \text { for even } N_{F}^{A} \\ \frac{N_{F}^{A}+1}{2}+\sum_{d=1}^{d_{\max }} \Gamma\left(\frac{N_{F}^{A}+4^{d}}{2^{2 d+1}}\right), & \text { for odd } N_{F}^{A}\end{cases}
$$

Here $\Gamma(a / b)$ is the quotient of the division of the variable $a$ by $b$, and $d_{\max }$ is the greatest dividing exponent of a base 4 with respect to $N_{F}^{A}$, i.e., $4^{d_{\text {max }}} \leq N_{F}^{A}$, and $4^{d_{\max }+1}>N_{F}^{A}$. As $N_{F}^{A}$ is 40 , thus $d_{\max }$ is 2 for this case $\left(4^{2} \leqslant 40\right.$ and $4^{3}>40$ ). Since $N_{F}^{A}$ in our case is an even number 40 , we can deduce using Eq. 5.14 that for a data rate of $25 \mathrm{kbps}$, at most 26 frequency offsets are usable in simultaneous communications within the 40 available frequency offsets. We use this parameter value of the available frequency offsets to calculate the throughput and efficiency of the frequency offset based multiple access model. 


\subsubsection{Performance of frequency offset based multiple access}

In this subsection, we present the performance evaluation results in terms of the throughput and the efficiency for the frequency offset based multiple access scheme for different limitations on the maximum number of simultaneous transmissions, $m$, within the acceptable interference level. The throughput and the efficiency results are obtained by varying the offered traffic for analytical modelling using Matlab, as presented in Section 5.3, and simulation results using $\mathrm{OMNeT}++$. Here we present the results for number of frequency offsets $C=5$ and $C=25$ to show how the throughput and the efficiency changes, when the number of frequency offsets in the system is increased from $C=5$ to $C=25$. We choose $C=25$, since this is a realistic value for the number of frequency offsets with the given parameters, as calculated in Subsection 5.4.1.

\subsubsection{Throughput}

We present the results for the throughput for frequency offset based multiple access model in this subsection, which is explained in Section 5.3, Eq. 5.6. The throughput is presented with respect to the offered traffic variation for number of frequency offsets $C=5$ and $C=25$ in Fig. 5.1 and 5.2 respectively. For number of frequency offsets $C=5$ in Fig. 5.1, we see a significant linear increase of throughput at low offered traffic load with increasing number of simultaneous transmissions, $m$. After reaching the maximum throughput at an optimized traffic level, the throughput decreases with increasing traffic load, due to many collisions caused by the overload of the system. The throughput goes to zero for large values of channel traffic. We see similar behavior in Fig. 5.2 for $C=25$. Note that the scaling in the y-axis for throughput results is different for different number of frequency offsets of $C=5$ and $C=25$ cases. The simulation results with $95 \%$ confidence interval confirm the analytical results. The confidence interval is very small because of the scaling in the y-axis.

We see a significant increase in the total throughput of the system by comparing the throughput of frequency offsets $C=5$ and $C=25$. The throughput increases as more offsets are added to the system, even for the same number of 


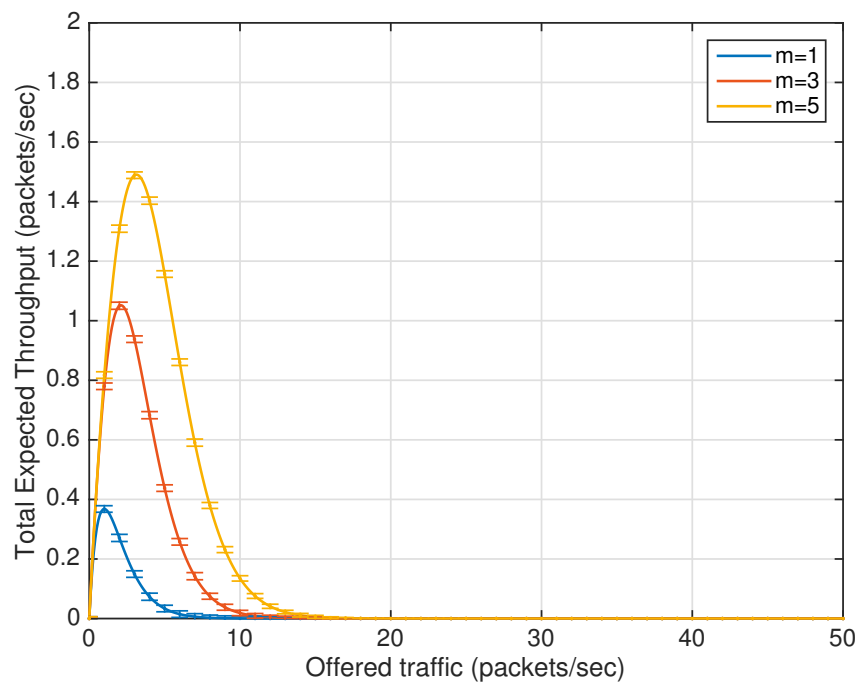

Figure 5.1: Throughput of the frequency offset based system with increasing number of frequency offsets, $\mathrm{C}=5$

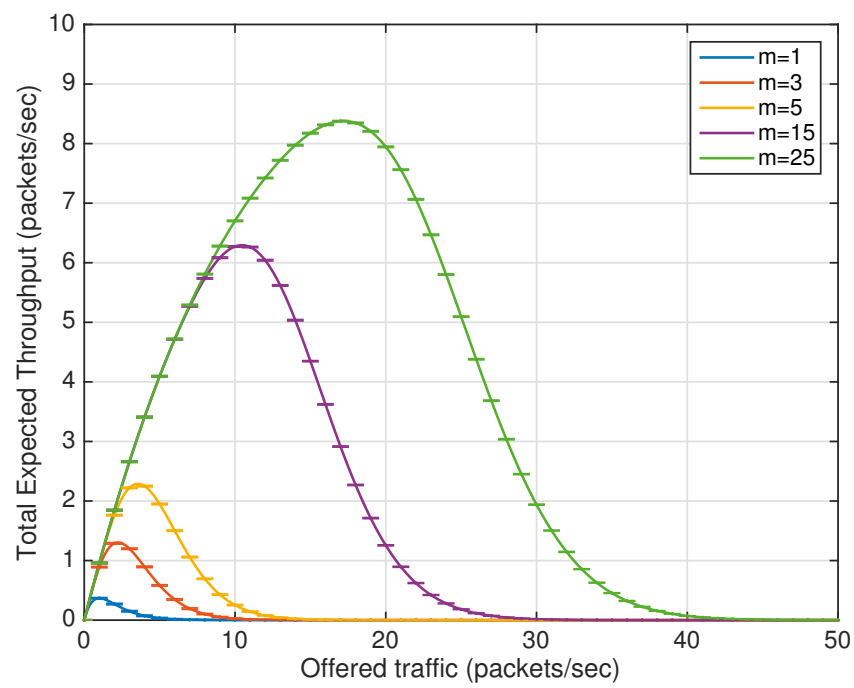

Figure 5.2: Throughput of the frequency offset based system with increasing number of frequency offsets, $\mathrm{C}=25$ 
simultaneous communications limitation. For example, the highest throughput peak with $m=5$ is at 1.5 packets/second for $C=5$ in Fig. 5.1, whereas the highest throughput peak with $m=5$ is at 2.2 packets/second for $C=25$ in Fig. 5.2. Furthermore, we see in Fig. 5.2 for $C=25$ that the throughput increases a significant amount for higher number of available frequency offsets when more simultaneous communications are allowed, for example, $m=15$ or $m=25$ compared to a smaller value of $m$.

\subsubsection{Efficiency}

We present the efficiency results for the frequency offset based multiple access scheme by varying the offered traffic load in Fig. 5.3 and 5.4 for frequency offsets $C=5$ and $C=25$, respectively. We present the efficiency for different values of limitations on the maximum number of simultaneous transmissions, $m$. To calculate efficiency, we normalize the throughput with respect to the number of simultaneous transmissions allowed, as presented in Section 5.3, Eq. 5.7. The simulation results with $95 \%$ confidence interval are also presented with the analytical results, which match with the analytical results. We see a linear increase in efficiency for low offered traffic load in both of the figures, which slows down after reaching a maximum point with increasing traffic load, as seen for the throughput case. The efficiency goes to zero at high traffic load. Here we also see that the shapes of the curves in both Fig. 5.3 and 5.4 correspond to the characteristic behaviour of slotted Aloha. We observe that $m=1$ results in the single channel S-Aloha system, because only one simultaneous communication is allowed. This also provides basic validation of our model as a generalization of S-Aloha.

Furthermore, we see in Fig. 5.3 that the maximum efficiency decreases for the number of available frequency offsets $C=5$ as the number of allowed simultaneous communications value $m$ increases. For example, the efficiency for $m=5$ is less than the efficiency for $m=1$. Efficiency decreases with the increasing the limitation on simultaneous communications, because the efficiency is calculated by normalizing the throughput by the number of maximum allowed simultaneous communications limitation. However, we observe in Fig. 


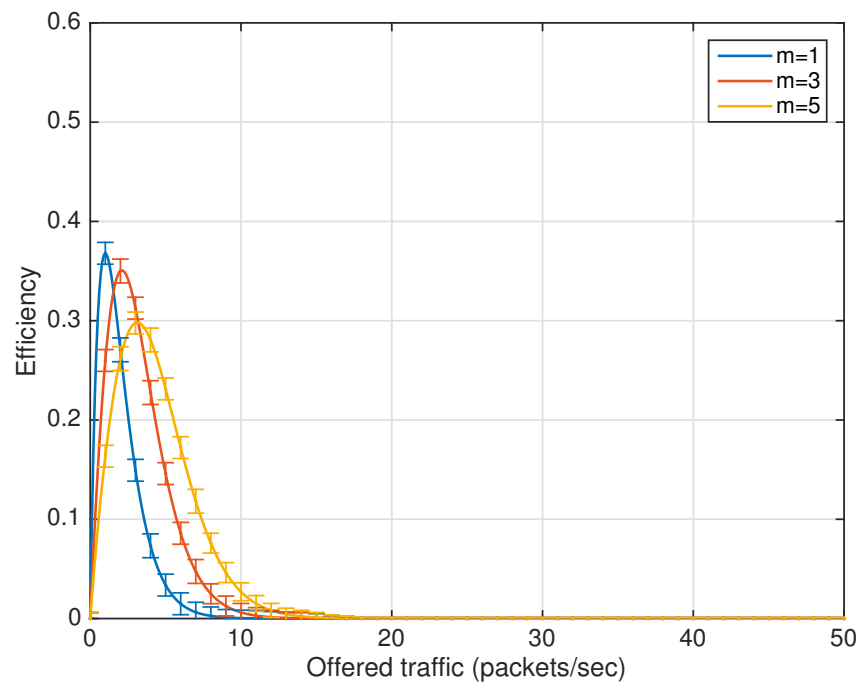

Figure 5.3: Efficiency of the frequency offset based system with number of frequency offsets, $\mathrm{C}=5$

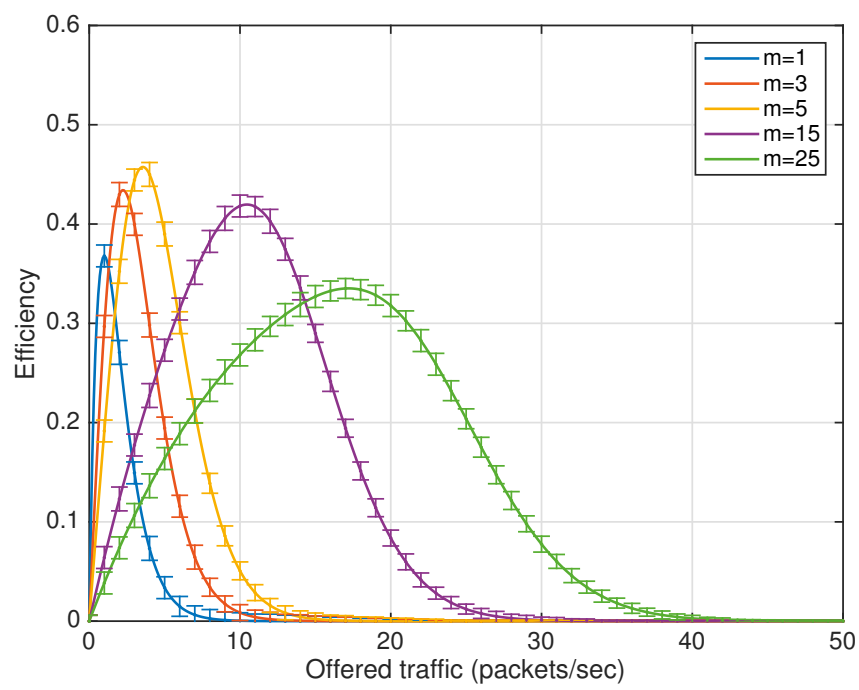

Figure 5.4: Efficiency of the frequency offset based system with number of frequency offsets, $\mathrm{C}=25$ 


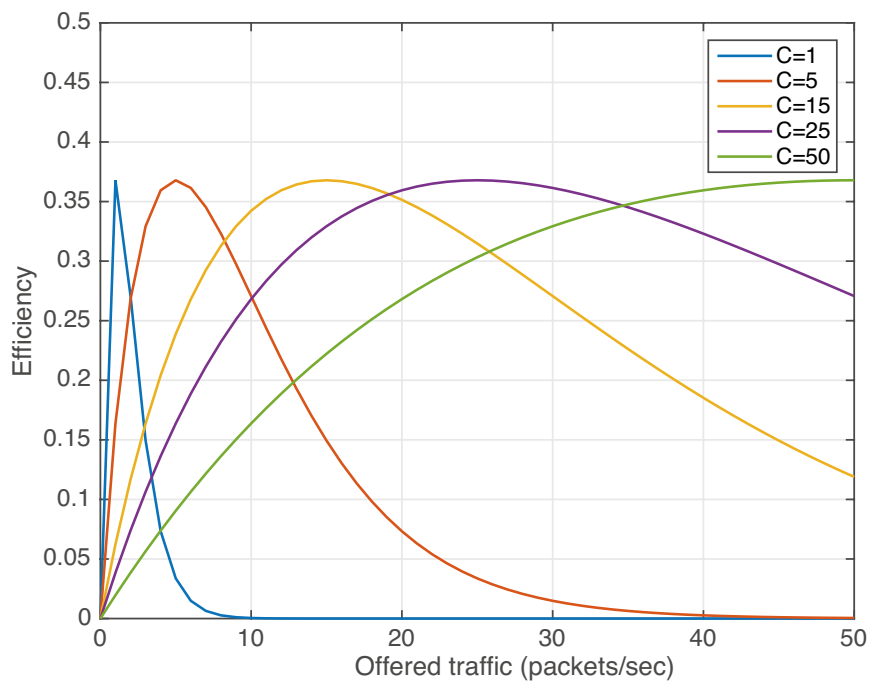

Figure 5.5: Efficiency of multi-channel S-Aloha

5.4 that the maximum efficiency increases when the number of frequency offsets increases $(C=25)$ for the same value of allowed simultaneous communications of $m=5$. The reason is that the probability of having a successful communication increases with the increase of more available frequency offsets to choose from the frequency offset pool. As a result, we observe the maximum efficiency exceeds the theoretical maximum of S-Aloha for a higher number of frequency offsets available in the pool, when a moderately low number of simultaneous communications are allowed ( $m=2,3,4)$. The maximum efficiency eventually decreases with imposing higher limitations on the number of simultaneous communications, for example $m=25$, with increasing traffic load.

\subsubsection{Performance of multi-channel S-Aloha}

As an intermediate step, we have obtained a model for multi-channel S-Aloha. Here we present its performance in terms of efficiency for several numbers of 
available channels by varying the offered load in Fig. 5.5. The corresponding analytical model is given in Section 5.3, Eq. 5.5. We see that the shapes of the curves correspond to the multi-channel S-Aloha. When a single channel is available for $C=1$, the system successfully corresponds to the single channel S-Aloha system along with its maximum efficiency of $37 \%$. Even though the number of frequency offsets increases, the efficiency maintains its maximum efficiency value. Furthermore, the position of the maximum efficiency point corresponds to the offered load, that is, $C=25$ provides the highest efficiency when offered load is 25 packets/sec. It means that a multi-channel system with 25 channels provides its highest efficiency, when 25 packet transmissions are taking place in 25 different channels. Therefore, the results correspond to what can be intuitively expected in a multi-channel S-Aloha system.

\subsection{Summary}

In this chapter, we propose a model for multiple access for frequency offset based transmit reference modulation extending a general model of multichannel S-Aloha based multiple access, where a successful communication implies that a unique offset is chosen, and the total number of simultaneous communications is below the interference limit. This multiple access model provides the fundamental insight into the performance of a MAC protocol that exploits the frequency offset based TR modulation in the physical layer. We find that the frequency offset based system provides much higher throughput and an increased efficiency compared to the multi-channel S-Aloha system under similar conditions. 


\title{
TR-MAC protocol: detailed design and performance analysis
}

\begin{abstract}
A MAC protocol controls the access of the shared wireless medium for the competing nodes of a wireless sensor network. Hence, the MAC protocol tries to ensure that the transmissions of multiple nodes do not interfere each other in a multiple access scenario. Using frequency offset based transmit reference modulation in the underlying physical layer can have advantages in the upper MAC layer for multiple simultaneous communications using multiple frequency offsets. Hence, a medium access control for a frequency offset based system is needed in the MAC layer. Earlier in Chapter 5, we proposed a multiple access model for multi-channel medium access control using frequency offsets, and analyzed its performance potential at the MAC layer based on the publication [23]. In this chapter, we extend the energy-efficient medium access control TRMAC protocol, introduced in Chapter 3, based on the publications [19, 20, 21], for the multiple access control with frequency offset based transmit reference modulation, and analyze its performance in a wireless sensor network with multiple access scenario. We also compare the performance results of the complete multiple access protocol with the results obtained using the multiple access model in Chapter 5.
\end{abstract}

The frequency offset based transmit reference modulation provides multiple frequency offsets, which can be utilized by multiple nodes to access the shared medium. Hence, a novel medium access control protocol for the multiple access scenario is required to address the medium access mechanism using multiple 
frequency offsets provided by the frequency offset based transmit reference modulation. To address this issue, the TR-MAC protocol takes the benefit of the underlying frequency offset based transmit reference modulation by enabling multiple pairs of nodes to choose from multiple available frequency offsets. As a result, multiple simultaneous communications can take place using different frequency offsets. Furthermore, the duty cycling mechanism of the energyefficient TR-MAC protocol enables a node to spend in low power sleep mode for most of its operational time, and wake up periodically to check for any ongoing activity in the channel. Hence, for a low data rate operation, only a few of the available frequency offsets are used simultaneously. Apart from the advantage of providing multiple frequency offsets for multiple communications, this frequency offset based TR modulation has some difficulties, such as, limited number of offsets and limited number of simultaneous communications [17]. The total number of available frequency offsets in the system is limited, because a frequency offset has to be smaller than the channel coherence bandwidth [15]. Moreover, the number of the simultaneous communications in the system also is limited, because of the increase in the multi-user interference in the presence of a large number of active links with multiple frequency offsets and self-mixing of the reference and the information signals [87]. Therefore, the performance benefit of the TR-MAC protocol using multiple frequency offsets for multiple access has a maximum limit due to the two mentioned limitations. In Chapter 5, we proposed a multiple access model taking into account these two limitations of TR modulation, and analyzed the performance of that hypothetical protocol using a mathematical model. In this chapter, we define a complete multiple access protocol, and analyze its performance using simulation.

This chapter expands the answer to the research question 5 ("How can an energy-efficient MAC protocol for WSNs be designed using transmit reference modulation for multi-channel medium access control using frequency offsets?"), as mentioned in Chapter 1. The contributions of this chapter are as follows: (1) we present a detailed design of the TR-MAC protocol, propose different communication strategies to establish synchronized link state, and present a state diagram for link state changing; (2) we analyze different aspects of the 
TR-MAC protocol from the multiple access perspective, which includes carrier sensing, exposed terminal problem, hidden terminal problem, collision and retransmission; (3) we present a finite state diagram for the TR-MAC protocol operation for multiple access scenario; (4) we analyze the TR-MAC protocol performance in a multiple access scenario.

This chapter is organized as follows: the detailed design of TR-MAC protocol is presented in Section 6.1 with different synchronized link establishment strategies to establish the synchronized link state. This section also presents a state diagram for the link state changing, which explains the procedure of the TR-MAC protocol to switch between its operational states. The design aspects for the TR-MAC protocol from the multiple access perspective is analyzed in Section 6.2, which deals with the critical issues related to multiple access, e.g., carrier sense, multiple simultaneous communications, hidden terminal issue, and collision and retransmission. This section also presents the finite state diagram for the protocol operation from a multiple access perspective. Subsequently, Section 6.3 presents the simulation setup and results, then analyzes the performance of the TR-MAC protocol for multiple access scenario in a wireless sensor network. Finally, the contents of this chapter are summarized in Section 6.4 .

\subsection{TR-MAC protocol: detailed design}

The TR-MAC protocol is a preamble sampling based energy-efficient duty cycling protocol for the frequency offset based transmit reference modulation, which is presented in Chapter 3. The TR-MAC protocol enables the nodes to choose from the multiple available frequency offsets provided by the frequency offset based transmit reference modulation in the underlying physical layer. In this section, we explain the design aspects of the medium access control using the TR-MAC protocol with respect to the multiple access scenario using multiple frequency offsets.

The TR-MAC protocol associates the use of multiple frequency offsets with its three operational states: first time communication, the unsynchronized 
link state, and the synchronized link state, as presented in Chapter 3. The operation in the first time communication also takes place in the same way as the unsynchronized link state. The unsynchronized link state uses a designated frequency offset, which is referred to as the default frequency offset. All the other frequency offsets are available in the frequency offset pool to be used by different nodes during their synchronized link state operation. Figure 6.1 represents the TR-MAC protocol operation for the unsynchronized and synchronized link states. In the beginning, all the nodes start their periodic listening operation in the unsynchronized link state with the default frequency offset to search for any ongoing activity in this common channel, and sleeps the rest of the time. The duration between the two consecutive periodic listen cycles is referred to as the check interval, which has a network-wide known maximum value. To perform a successful communication, one transmitter node transmits preamble-data, and waits for the acknowledgement, and repeats this procedure until the maximum check interval duration, or until an acknowledgement is received from the desired receiver. If the transmitter and receiver node agree on a frequency offset and a time instance for their future communication, then they move to the synchronized link state. In this way, all the nodes try to make pairs during the operation in the unsynchronized link state with the default frequency offset, so that they can operate using a different frequency offset using the synchronized link state.

The protocol operation using the synchronized link state has two advantages. Firstly, the synchronized link state operation is energy-efficient, because the transmitter can optimally minimize the preamble-data and acknowledgement listen cycle duration to as small as possible for the future communications, and the receiver can minimize its idle listening duration. Secondly, one pair of nodes can use a previously agreed frequency offset in the synchronized link state, which is different than the default frequency offset used in the common channel. Since there are many frequency offsets available, therefore this pair of nodes experiences less contention when they want to access the channel. This also results in a less contention in the default channel, because a pair of nodes offloads themselves from the default channel after setting up a synchronized 


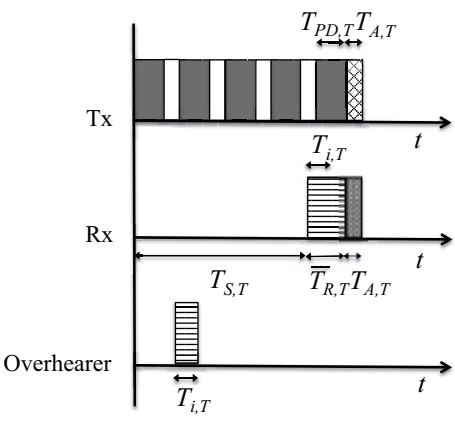

Unsynchronized Link State

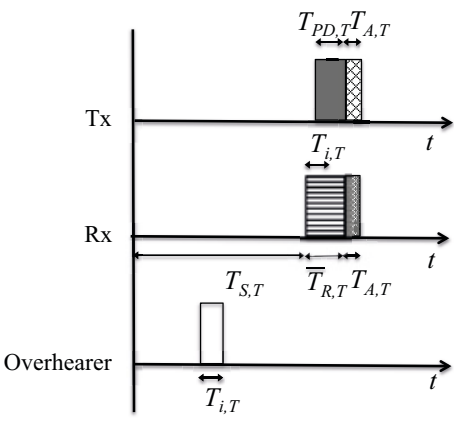

Synchronized Link State

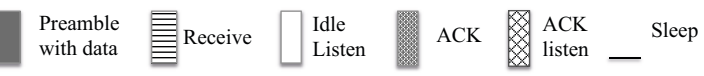

Figure 6.1: TR-MAC protocol operation

link state between them.

Each node in the network has to maintain a data structure to keep track of its send and receive events, and its neighbors. Furthermore, one node has to remember the particular link state for each of its neighbors. The frequency offsets are related with the link states. One node has to remember the frequency offset, and the particular time instance for communicating using the synchronized link state with its neighbor pair. This data structure is explained in detail in Subsection 6.1.3.

One important consideration in this link state based operation of the TR-MAC protocol is that one node has to perform communication in both unsynchronized and synchronized link states during its protocol operation. A node communicates in the synchronized link states with its synchronized pairs at a particular frequency offset and a particular time instance. Moreover, this node also needs to continue its periodic listening in the default frequency offset in the search of new neighbors. Furthermore, this node may require sending a packet to a new neighbor in the unsynchronized link state, with which the synchronized communication link is not yet established. In this case, this node requires to send packets in the unsynchronized link state for a maximum of the check interval 
duration. As a result, this node has to postpone its periodic listening and all the synchronized communications with all its pairs for a maximum of the check interval duration, when it needs to send a packet using the unsynchronized link state with the default frequency offset. If the potential receiver node is also sending in the unsynchronized link state during the same check interval by also canceling its periodic listening and other synchronized communications, then the transmitter node cannot reach this potential receiver node during this check interval. Since this transmitter node has to resume its periodic listening in the default frequency offset and the synchronized communications with its pairs, thus the transmitter node performs its next transmission attempt using the unsynchronized link after a random multiple of the check interval duration. After a failing attempt to reach a potential receiver node during an unsynchronized link state sending operation, the transmitter node does not attempt to transmit immediately for the next check interval duration in order to avoid a repetition of the event that prevented the successful communication during the last attempt. The communication in the unsynchronized link state using the default frequency offset consumes more energy compared to the synchronized link state; because of the long iteration of preamble-data packet and acknowledgement listen cycles. Failing to communicate with a synchronized pair using a previously agreed frequency offset for a number of attempts results in the pair falling back to the unsynchronized link state with the default frequency offset. Furthermore, higher number of nodes communicating in the unsynchronized link state make the default frequency offset crowded. As a result, it is recommended to maintain the already established synchronization between pairs of nodes by restraining quick falling back to the unsynchronized link state.

In this section, we analyze the protocol operation of the TR-MAC protocol in detail, which was first introduced in Chapter 3. In particular, we present the design choices for different synchronized link establishment strategies of the TR-MAC protocol using multiple frequency offsets. Afterwards, we present the state diagram for link state changing to move between the operating states of the TR-MAC protocol, namely the unsynchronized link state, and the synchronized link state. Furthermore, we explain about the bookkeeping procedure that one 
node uses to save information about the frequency offset and the time instance of its future activities for its own periodic listening and for its neighbor pairs. Finally, we present the information that the nodes require to exchange in the header part of the preamble-data or the acknowledgement packet.

\subsubsection{Synchronized link establishment strategies}

The synchronized link state communication can be established from the unsynchronized link state in two ways, i. receiver-driven strategy, and ii. transmitterdriven strategy. We explain these two strategies in the next subsections.

\subsubsection{Receiver-driven communication}

In the receiver-driven communication strategy to establish the synchronized link state from the unsynchronized link state, the receiver node allocates a frequency offset from the pool of available frequency offsets, and a time instance for this particular transmitter node in order to make a pair with it. As the receiver node controls the allocation of the resources, thus this strategy is referred to as the receiver-driven communication strategy. After allocating the resources and saving them in its data structure, the receiver node informs about the frequency offset and the time instance to its transmitter pair using the acknowledgement message. The transmitter node receives this information after receiving the acknowledgement packet, and saves them in its data structure, provided that there is no overlapping with any other activity in that allocated time instance. This handshaking process is further explained in Subsection 6.1.2.

The advantage of this receiver-driven communication strategy is that the receiver can randomly assign an unused frequency offset and corresponding time instance by checking the availability in its own data structure of the neighbor list. This data structure is presented in Subsection 6.1.3. In this way, the receiver node can have control about the transmitter pairs that it accepts. This in turn helps the receiver to allow many transmitter pairs to send to this particular receiver node within one check interval duration. 


\subsubsection{Transmitter-driven communication}

In this subsection, we explain the transmitter-driven communication strategy to establish a synchronized pair. Using this strategy, one node requests its neighbor node to wake up at a particular frequency offset and a particular time instance for their future communication. This transmitter-driven request can be communicated to the desired receiver during their unsynchronized communication.

This transmitter-driven communication strategy can be useful if one node having less energy can transfer the operational burden to its neighbors by requesting them to follow its lead. In that case, the node pair can operate in an energydriven manner, because the transmitter node saves energy by sending one preamble-data packet. The transmitter-driven strategy can also be useful to perform a broadcast communication to its neighbors in an energy saving manner. In a normal broadcast operation, one transmitter sends data packets using the default frequency offset. With the transmitter-driven communication strategy, this node requests its neighbors to add an extra listen cycle in a particular frequency offset, and send one preamble-data packet at that specific time instance. The corresponding receiver nodes allocate this extra listen cycle at that particular frequency offset and wake up at the corresponding time instance. If this transmitter-driven requested activity conflicts with any other synchronized operation in a receiver node or with the periodic listening in the default frequency offset, then the receiver node prioritizes the broadcast listening over its other activities.

This transmitter-driven communication strategy using the synchronized state for broadcast communication can have several advantages compared with a broadcast communication using the unsynchronized link state. Firstly, a communication using the unsynchronized link state means sending many iterations of preamble-data packets for a maximum of check interval duration, which consumes higher energy compared to a synchronized transmitter-driven communication. Secondly, the default frequency offset with the unsynchronized link becomes completely busy for the check interval duration for a broadcast operation. If the broadcast communication can be performed using a particular 
frequency offset, then the number of contentions in the default frequency offset can be minimized. Finally, the use of transmitter-driven strategy can provide some interesting opportunities to realize energy-efficient multi-hop communications at the network level. For instance, TR-MAC is able to create a ripple effect for broadcasting while maintaining its energy-efficiency. The transmitter can instruct its first hop neighbors to follow its lead, and those can in turn instruct their respective neighbors to follow them in order to broadcast more efficiently, thus saving both energy and time. As a result, a system of Green Waves [88] can be created to deliver packets to their destinations with limited delay. The limitation of this approach is that the neighbor nodes have to assign priorities, if this extra transmitter-driven listen cycle's frequency offset or time instance conflicts with any of its previously agreed synchronized pairs or with its periodic listening in the default frequency offset.

\subsubsection{State diagram for link state changing}

In this subsection, we present a state diagram for the link state changing for the TR-MAC protocol to switch between the unsynchronized link state and the synchronized link state. One node using the TR-MAC protocol needs to change between these two operational link states when necessary. Since the synchronized link establishment requires two nodes to form a pair, thus we present the state diagram from the point of view of two nodes. Any node can initiate the request to move to the synchronized link state. TR-MAC offers two communication strategies with receiver-driven communication and transmitter-driven communication. Hence, this state diagram can be applied to both receiver-driven and transmitter-driven communications strategies. We do not distinguish between a transmitter node and a receiver node. Rather, we distinguish the two nodes as the initiator node of the synchronized link establishment process, and the remote node that receives the synchronized link establishment request.

Figure 6.2 represents the state diagram for link state changing for the TR-MAC protocol. Here one node initiates the synchronization process, which is referred to as the initiator node state; and another node that receives the synchronization 


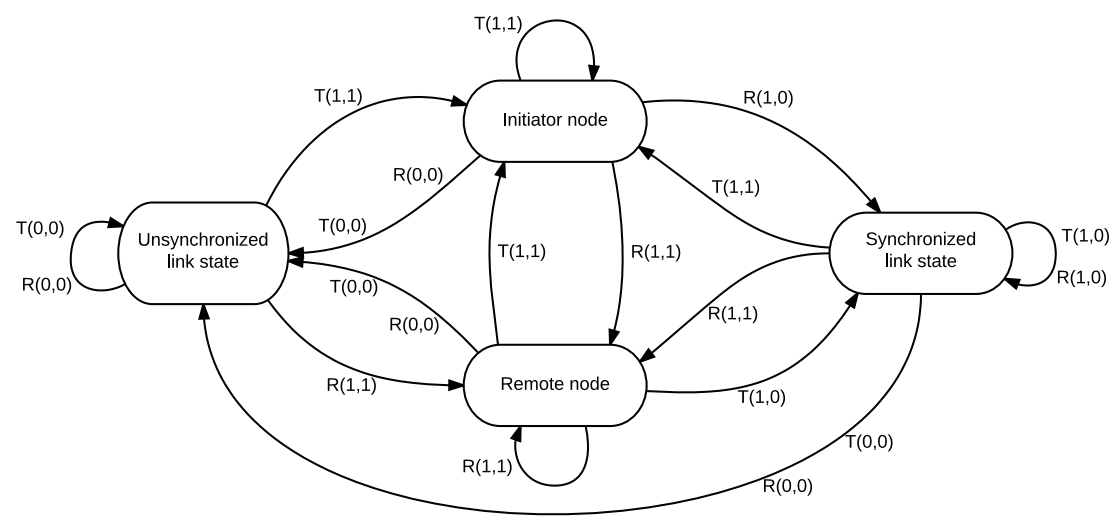

Figure 6.2: State diagram for link state changing for the TR-MAC protocol

request is referred to as the remote node state. Both of these two nodes initially operate in the unsynchronized link state, and their final goal is to reach the synchronized link state, as referred in the figure. Each of the two nodes has an operational version of the state diagram for the handshaking process to move between these two operating states. State transition happens based on the transmission or the reception of a message. In these transitions, the character $\mathrm{T}$ refers to the transmission, and the character $\mathrm{R}$ refers to the reception of a message. Two extra bits are used in the header section to specify the exact actions, namely the propose-to-sync-bit represented as the first digit, and the frequency-offset-propose-bit represented as the second digit. The propose-to-sync-bit is used to indicate a proposal to move to the synchronized link state from the unsynchronized link state. One initiator node sets the propose-to-sync-bit to 1 in the header part, if it is willing to establish a synchronized link state with the remote node. The frequency-offset-propose-bit is used to indicate a new frequency offset. The initiator node sets the frequency-offset-propose-bit to propose that a new frequency offset is specified to the remote node; where representing the actual frequency offset requires few extra bits in the header part. The header part with its extra bits is elaborated in detail in Subsection 6.1.3.

Initially both of the nodes communicate in the unsynchronized link state. When- 
ever one node wants to move from the initiator node state to the synchronized link state, it sets the propose-to-sync-bit to 1 , and also sets the frequency-offsetpropose-bit to 1 ; which results in the transition represented by $\mathrm{T}(1,1)$ from the unsynchronized link state to the initiator node state. This initiator node now waits for the response from the remote node. The remote node receives $R(1,1)$, which is represented by the transition from the unsynchronized link state to the remote node state. The remote node now has to decide among three options. i) The remote node refuses the proposal to move to the synchronized link state and moves back to the unsynchronized link state. This transition is represented by $\mathrm{T}(0,0)$ from the remote node state to the unsynchronized link state. The initiator node receives this denial message from the remote node. As a result, the initiator node moves back to the unsynchronized link state, which is represented by the transition $\mathrm{R}(0,0)$ from the initiator node state to the unsynchronized link state. ii) The remote node accepts the state changing request, and agrees with proposed frequency offset. Therefore, it moves to the synchronized link state and sends $\mathrm{T}(1,0)$ to the initiator node, which is represented by the transition from the remote node state to the synchronized link state. Here the frequency-offset-propose-bit is not set, which represents the fact that the proposed frequency offset is ok with the remote node. The initiator node receives the acceptance message, and also moves to the synchronized link state; which is represented by $R(1,0)$ from initiator node state to the synchronized link state. iii) The remote node accepts the request to move to the synchronized link state, but does not agree with the proposed frequency offset. Hence, the remote node proposes a new frequency offset by setting the frequency-offset-propose-bit; and waits for the response from the initiator node. This transition is represented by $\mathrm{T}(1,1)$ from the remote node state to the initiator node state. On the other hand, the initiator node receives this new proposal, which is represented by $\mathrm{R}(1,1)$ from the initiator node state to the remote node state. As can be observed in the state diagram, now the role of the initiator node and the remote node has been switched; and the new remote node now has to decide from the three options mentioned above.

If two nodes operating in the synchronized link state after forming a pair want to switch their frequency offset, then the initiator node sets both the propose-to-sync- 
bit and the frequency-offset-propose-bit. This transition is represented by $\mathrm{T}(1,1)$ from the synchronized link state to the initiator node state. The remote node receives $R(1,1)$, which is represented by the transition from the synchronized link state to the remote node state. Then the decision making process would be performed by the initiator and remote node in the process mentioned in the last paragraph. The self-loop in the unsynchronized link state with transition $\mathrm{T}(0,0)$ and $\mathrm{R}(0,0)$ means that both the nodes want to continue operation in the unsynchronized link state. Similarly, the self-loop in the synchronized link state with the transition $\mathrm{T}(1,0)$ and $\mathrm{R}(1,0)$ means that both the nodes would like to continue with the current synchronization pattern, and agree with the current frequency offset. The transitions $\mathrm{T}(0,0)$ and $\mathrm{R}(0,0)$ from the synchronized link state to the unsynchronized link state means that both the nodes want to discontinue the synchronized operation.

There could be packet loss in the synchronized link establishment process. In the case of packet loss, the initiator node has to repeat the proposal to move to the synchronized link state after a timeout event, which is represented by the self-loop in the initiator node state with the transition $T(1,1)$. Similarly, the self-loop in the remote node state with the transition $\mathrm{R}(1,1)$ represents the timeout event in the remote node caused by a packet loss. After a number of retransmission attempts, the initiator node falls back to the unsynchronized link state, which is represented by the transition $\mathrm{T}(0,0)$ from the initiator node state to the unsynchronized link state. The collision and retransmission in the unsynchronized and synchronized link state is analyzed further in the Subsection 6.2.4.

\subsubsection{Information bookkeeping and exchange}

To perform the TR-MAC protocol operation, each node needs to maintain a data structure to store the frequency offset and the time instance after making a pair with one of its neighbor nodes to operate in the synchronized link state to communicate in future. This data structure is organized in a time-sorted manner such that it keeps track at the next event, which can be a periodic listening in the default frequency offset after the check interval duration, or a 
synchronized wake up or a synchronized packet sending time for a paired node at a particular frequency offset. One node schedules the next event before going to the sleep mode. When this node wakes up again, then it performs the event by checking that data structure. Before adding a new entry for a pairwise node in the data structure, the current node has to check for frequency offset or time overlapping event in the data structure. The node adds a new entry only if there is no overlapping event in the data structure. The periodic listening activity in the default frequency offset for each node is the highest prioritized event in order to facilitate the information receiving from other nodes, which is the basic requirement for a preamble sampling protocol.

This data structure to keep track of the activities and information in a timesorted manner, which includes the following: the node identifier, the event whether it is a send or a receive event, current communication link state with this node, the frequency offset, starting time of the event, and the duration of the event. We discuss about the node identifier at the end of this subsection. This data structure is time-sorted based on the events to perform next. The first event is the periodic listening time instance with the default frequency offset for each node itself. One node inserts a new entry after communicating with a neighbor node by specifying the send or receive event and current state of communication.

For the protocol operation, some information needs to be exchanged in the header part of the preamble-data packet or the acknowledgement packet. The information that needs to be exchanged are as follows: the node identifier, the propose-to-sync-bit to indicate a proposal to move to the synchronized link state if it is set, the frequency-offset-propose-bit to indicate that a new frequency offset is proposed if it is set, optionally the frequency-offset bits to represent the actual frequency offset, and the more-bit to represent that more data packets are coming from the sender node towards the current receiver node. The node identifier is further discussed at the end of this subsection.

Transmitting the frequency offsets takes few bits in the header of the acknowledgement packet, since there is a finite number of frequency offsets in the system. However, mentioning the exact time instance with the precise hour, 
minute, and second information would require many bits. Therefore, we divide the base check interval by the number of available frequency offsets, and bind the possible time offsets with the frequency offsets. Hence, communicating only the frequency offset is enough for a node to derive the related time instance. In this way, one receiver node can communicate the chosen frequency offset to its sender pair, and the sender node can derive the time instance from the given frequency offset and the time of the base check interval. In both of these nodes, the future communicating time instance can be calculated by adding the time offset together with the base check interval duration based on the last successful communication. Hence, the header part does not need to include the time instance for the future communication, and saves energy by reducing the communication overhead. As a result of binding the frequency offset together with the time offset, one node can allocate as many synchronized pair nodes as the number of available frequency offsets in the system within its one check interval duration. This node communicates with its remaining neighbors using the unsynchronized link state on the default frequency offset.

One important consideration in this binding is that the check interval of the nodes needs to be the same to derive the exact time offset from the frequency offset. In Chapter 3, we proposed an analytical model to optimize the check interval of the system for the unsynchronized link state operation. After finding an optimized check interval for a particular operation scenario, that check interval value can be tuned in as the maximum check interval duration of the network. Recall that, the maximum check interval duration needs to be known in the network for a transmitter node to be able to stop its preamble-data sending, and acknowledgement listening cycle iteration for the unsynchronized link state operation.

In Chapter 4, we presented a traffic-adaptive duty cycle adaptation algorithm, where a receiver node increases its duty cycle by waking up at a higher rate for increasing traffic, and decreases its duty cycle by removing the extra listen cycles for decreasing traffic. The sender pair uses the same algorithm to estimate the extra listen cycles of its receiver pair to send packets when the receiver pair wakes up. These extra listen and send cycles needs to be maintained in the event 
list for both of the nodes in the pair. However, increasing the communicating frequency with a node pair is limited to the available non-overlapping time instances that this node can allocate. In case of overlapping time instances, one node needs to provide priority between its normal operation and trafficadaptive scheme with its pairs.

The node identifier can be the MAC address, which is a 48 bits long globally unique identifier assigned to the sensor nodes in a network. However, exchanging 48 bits in the header part of the preamble data or the acknowledgement packet imposes too much extra overhead, if only locally unique address of the sensor nodes are capable of delivering the same outcome. In order to reduce the extra overhead of addressing, some solutions in the literature enable sensor nodes to have pre-assigned locally unique addressed before deployment, e.g.; Berkeley Motes [89]. Some other solutions enable the sensor node to assign locally unique addresses when they join the network, e.g., ZigBee [44], PACMAN [90]. The authors in [91] present a locally unique dynamic address allocation algorithm with duplicate address detection, which takes less energy to maintain the addressing. The authors in [92] surveys the addressing algorithms for wireless sensor networks, which are used to minimize the overhead related to addressing of the nodes. By using the minimum number of bits for the node identifier to uniquely address all the nodes of the network can offer remarkable reduction in addressing overhead. For example, using only 8 bits as the node identifier allows to uniquely identify 256 nodes within a network. In practice, few addresses among these 256 addresses can be reserved for special addresses, for example, broadcast address. Depending on the maximum number of nodes in the network, the number of bits for the node identifier can be adjusted. This unique node identifier is used in the header of every packet to address another node instead of the exact address to minimize the number of bits required for node addressing. After establishing a synchronized link, two neighbors only need a minimal check about the node identifier to ensure that communications is indeed taking place with the intended neighbor. 


\subsection{TR-MAC protocol: multiple access perspective}

In this section, we discuss the consequences of using the unique TR modulation as the underlying physical layer and the unique TR-MAC protocol in the MAC layer from the multiple access perspective. The MAC protocol has the responsibility to provide an access mechanism to the shared wireless communication medium. At the same time, the MAC layer wants to minimize the probability of collisions in a wireless sensor network. Multiple nodes trying to access the shared wireless medium simultaneously may lead to a collision of packets. As a result, the desired receiver does not receive the transmitted packet, or the sender does not receive the acknowledgement. In this situation, the MAC protocol has to manage the retransmission, because the dropped or collided packet has to be retransmitted.

The TR modulation technique transmits the modulated signal together with the reference carrier signal using a frequency offset. Although one receiver node can decode only one signal at a time using a particular frequency offset known beforehand, it receives signals from the whole frequency spectrum. At the potential receiver, the other simultaneous communications using different frequency offsets in the shared frequency spectrum acts as interference, and increases the overall noise floor. One sender node also cannot transmit using two different frequency offsets simultaneously.

In this section, we present several scenarios where a collision can happen, and provide solutions to minimize the probability of collisions for the TRMAC protocol with frequency offset based TR modulation. We discuss about carrier sense, multiple simultaneous communications, hidden terminal problem, exposed terminal problem, and collision and retransmission issues. Finally, we present the retransmission mechanisms to handle a collision for the TR-MAC protocol at the end of this section.

\subsubsection{Carrier sense}

In general, the wireless communication medium is shared among the nodes of the WSN. As a result, one node already transmitting in the shared communica- 
tion channel occupies that channel. Collision happens if more than one node starts transmitting without checking whether the channel is currently busy or free. Therefore, carrier sensing can be performed in a node prior to transmit a packet to assess whether the channel is currently occupied by another user or not [93]. If the channel is assessed as free during carrier sense, then the node can transmit immediately. Otherwise, the node needs to backoff in order to avoid collision, if the channel is assessed as busy during the carrier sense.

TR modulation experiences overlapping frequency spectrum because of its design of transmitting the modulated signal using a frequency offset together with the reference carrier signal. One node can decode only one signal at a time using a particular frequency offset. However, the presence of many active simultaneous users using different frequency offsets in the shared frequency spectrum increase the overall interference and noise floor at the receiver. Therefore, the wireless channel using TR modulation can be interpreted as busy in two ways. Firstly, if another packet is received while listening to a particular frequency offset. Secondly, the received signal level during the channel sensing is very high, because of the interference level due to the simultaneous use of several other frequency offsets. Initiating a new transmission in this scenario might make the whole spectrum to collapse by increasing the total interference level of the channel to an unacceptable level to decode by any node. Hence, the node interprets the channel as busy based on the total interference level and received SNIR (signal to noise and interference ratio), even though no packet is received by listening to a particular frequency offset.

\subsubsection{Carrier sense duration}

The duration of the carrier sense has to be long enough to avoid any detection failure. To detect the data transmission in the medium and avoid any detection failure, taking into account the preamble sampling protocol proposed in Chapter 3 , where we take the duration of the periodic listening to be at least equal to the preamble duration for a first preamble-data packet, plus the duration for the acknowledgement reception, plus the preamble duration for a second preambledata packet. Since the purpose of the carrier sense is to detect data transmission 
in the medium without any detection failure, thus the carrier sense duration can be the same as the periodic listen duration. However, the periodic listening is extended after a communication is detected in the medium, which is not necessary for the carrier sense. Once the node learns that the channel is busy, then it can backoff. The carrier sense is performed when one node wants to send a packet with a purpose to backoff in order to avoid collision.

\subsubsection{Carrier sense with TR-MAC protocol states}

Using the TR-MAC protocol for the unsynchronized link state, nodes communicate using one particular frequency offset designated as the default frequency offset in the beginning of their protocol operation. All the nodes perform a carrier sense before sending a packet to the medium to prevent unnecessary collisions. Collisions are costly from the MAC layer protocol perspective, because a collision consumes energy for the receiver node and the sender nodes for an unsuccessful transmission attempt. Moreover, the sender nodes have to retransmit the collided packets to the desired receiver during the receiver's active time. One node performs carrier sense in the default frequency offset for the unsynchronized link state of the TR-MAC protocol, when it wants to send a packet. This node backs off with a random backoff timer, if it finds the medium is busy during the carrier sense. After scheduling the backoff timer, the node goes back to sleep to save energy. In 802.11 based WLAN protocol [94] and some other preamble sampling protocols, e.g., [39], the transmitter listens to the medium till the medium is free. This method is not suitable for the TR-MAC protocol, since extending the listen cycle in the unsynchronized link state for a node affects the other synchronized listen cycles already scheduled with its many pairs. Furthermore, this overhearing strategy consumes extra energy for the overhearing nodes. Hence, it is better to allow the transmitter to go back to sleep if it finds the medium is busy to increase the energy-efficiency. This node tries to send again when the backoff timer expires by performing carrier sense. If this node finds the medium is busy during the next carrier sense, then it again backs off. The duration of the backoff time in the beginning is chosen as a random fraction of the check interval time, since on average one node transmits 
only half of the check interval duration to successfully communicate a packet (See Chapter 3). Choosing a smaller backoff time avoids the collision, and at the same time allows the nodes to quickly move to the synchronized link state after a successful communication in the unsynchronized link state.

During the carrier sense in synchronized link state of TR-MAC protocol, one node performs carrier sense in the synchronized previously agreed frequency offset to communicate with one of its neighbors. If another node tries to use the same frequency offset at the same time, then the later node finds the medium is busy. Recall from the Section 6.1 that one receiver node allocates the new frequency offsets for its pairs based on its periodic listening time instance. Since the periodic listening of the all the nodes are uncorrelated and completely random, the probability of overlapping time instances for multiple nodes using the same frequency offset is low. However, if this situation occurs, the synchronized sender node holds its transmission by performing a backoff, goes back to sleep, and tries again for the receiver's next synchronized wake up time to avoid probable collision at that earlier moment. On the other hand, the synchronized receiver wakes up at the previously agreed time instance in the synchronized frequency offset only to receive nothing. Therefore, the synchronized receiver wakes up again after updating its next wake up time after the same interval. After a number of communication failures in the synchronized link state, this pair of nodes falls back to the unsynchronized link state.

\subsubsection{Exposed terminal issue}

The exposed terminal problem occurs in wireless networks when a node restricts its transmission to other nodes outside the collision region, because of overhearing an ongoing transmission of a neighbor node to the shared wireless medium during its carrier sense period. Consider the scenario in Figure 6.3 with four nodes, where the transmission range is also shown using circles around a particular node. Here node 3 is sending to node 4 , and node 1 receives that packet by overhearing during the carrier sense performed at the same time. As a result, node 1 delays its transmission to node 2, even though this communication does not interfere with the communication between node 3 and 


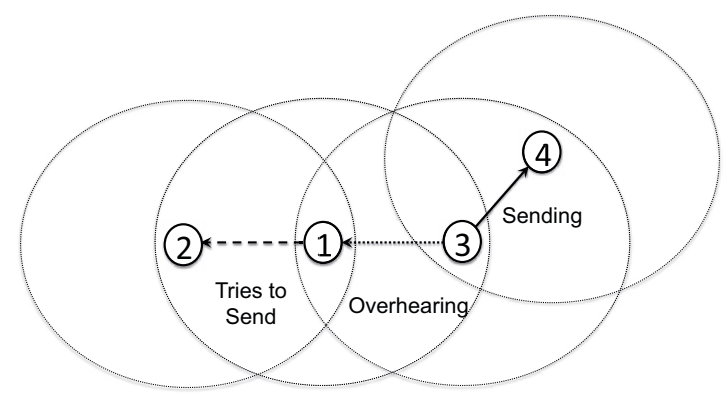

Figure 6.3: Exposed terminal issue

node 4 . This phenomenon is called exposed terminal problem, where a possible successful communication is delayed unnecessarily.

Using TR-MAC protocol in the unsynchronized link state, all the nodes in the figure are using the default frequency offset. In this scenario, node 1 can listen to the transmission of node 3 during its carrier sense. As a result, node 1 defers its transmission to node 2 , and backs off using the backoff timer. In the synchronized link state of the TR-MAC protocol, consider that the node 1 and 2 pair are synchronized using one previously agreed frequency offset, and node 3 is communicating with node 4 using the default frequency offset or another previously agreed frequency offset. In this case, node 1 does not overhear the communication from node 4 , because of using different frequency offset. However, the overall interference at node 1 during its carrier sense is higher. If the received signal to noise and interference ratio (SNIR) is below a certain threshold, then the channel is considered as busy. Considering a different scenario, where the node 1 and 2 pair uses the same frequency offset as the node 3 and 4 pair, then node 1 defers its packet transmission to the next synchronized wake up time of node 2. For the exposed terminal issue, the nodes does not change the frequency offset or the time instance. The protocol accepts that the exposed terminal issue can happen sometimes and the protocol looses capacity, which is better than sending a packet and cause a collision. 


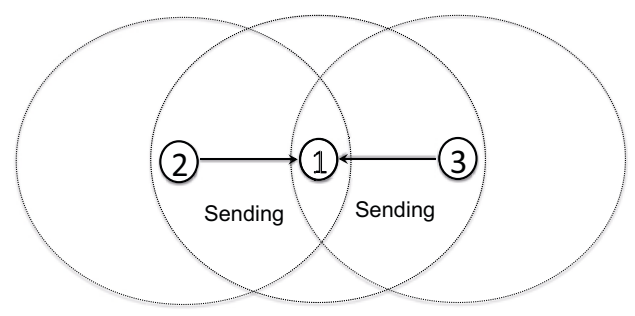

Figure 6.4: Hidden terminal issue

\subsubsection{Hidden terminal issue}

The hidden terminal problem occurs in wireless networks when nodes outside the range of each other communicate simultaneously to a single node, which is within the carrier sense range of those nodes individually. Consider a scenario in Figure 6.4 with three nodes, where the transmission ranges of these nodes are shown with a circle around a node. In this scenario, node 2 and 3 themselves are out of range of each other, but both node 2 and 3 are within the range of node 1 individually. In this case, a simultaneous transmission from node 2 and 3 towards node 1 , or towards some other nodes leads to collision at node 1 . This situation is referred to as the hidden terminal problem, where node 2 and node 3 are hidden to each other.

In the unsynchronized link state case for the TR-MAC protocol, the receiver node receives two garbled packets because of a collision. As a result, the receiver node does not send any acknowledgement back to the individual transmitter node. Eventually the transmitter node has to retransmit, if it does not receive an acknowledgement from the desired receiver node. On the other hand, if both the transmitter nodes do not receive any acknowledgement, then they continue sending preamble-data packets and wait for acknowledgement, and continue this cycle for a network-wide known maximum check interval duration. Eventually, this leads to a collision of all successive preamble-data packets incoming from these two transmitters. 
One solution to this hidden terminal problem is to use the three way handshaking RTS (request to transmit)/CTS (clear to transmit) mechanism, as used in MACA [95] and in IEEE 802.11 [94]. The preamble sampling B-MAC protocol proposes to solve the hidden terminal problem using RTS/CTS in the upper layer [38]. However, the author in [3] claims that, given the overheads involved in RTS/CTS signaling compared to the small WSN payloads, taking the resulting retransmissions for granted could be accepted as the best approach. Another approach to minimize the hidden terminal problem is to make the carrier sensing range larger than the collision range to minimize the collision probability of hidden terminals, as proposed in [50] and [96]. Carrier sensing range can be made larger than the collision range by using a sensing threshold far below than the receiving threshold. For example, a sensing threshold of $-100 \mathrm{dBm}$, which is far below than the receiving threshold of $-85 \mathrm{dBm}$, results in a larger sensing range than the collision range [50]. This approach minimizes the hidden terminal problem, but the increased carrier sensing range would result in higher exposed terminal problem, and higher number of backoffs. It might be the case that the node is backing off even though the other transmission would not interfere with its transmission.

During the synchronized communication using the TR-MAC protocol, simultaneous communications using different frequency offsets does not lead to the hidden terminal problem. Using the receiver-driven approach, one receiver does not allocate pairs if the time instance of two communications are overlapping, since one receiver can listen to only one frequency offset at a time. For example, if node 1 in Figure 6.4 allocates a time and frequency offset for node 2 , then node 1 does not allocate the same time for node 3. Furthermore, one receiver does not overhear the communication if one transmitter wants to send to a different receiver at the same time using a different frequency offset.

However, simultaneous communications using the same frequency offset results in a collision at the receiver for the TR-MAC protocol synchronized link state operation. In this case, the synchronized receiver marks one synchronized communication was missed. After a number of such misses, the receiver falls back to the unsynchronized link state. On the other hand, if the transmitter 
node does not receive an acknowledgement after sending a packet, it holds the packet retransmission and tries again at the next synchronized wake up time. The transmitter node also eventually falls back to the unsynchronized link state after a number of missed attempts during the synchronized link state operation. Although simultaneous transmissions using several different frequency offsets does not lead to hidden terminal problem, but they contribute to the overall interference in the frequency spectrum. With too much interference in the frequency spectrum, all the transmissions using different frequency offset will also be unsuccessful.

\subsubsection{Multiple simultaneous transmissions}

Consider a scenario where two nodes within range of each other start carrier sense at exactly the same time, and both of them find the medium is empty. As a result, both of the nodes start transmission simultaneously towards a receiver, which is also in the range of the two transmitter nodes. This situation leads to a collision at the receiver. Figure 6.5(a) represents this scenario with three nodes within the range of each other, where node 2 and node 3 both perform carrier sensing at the same time, find the medium is empty, and send simultaneously to node 1 . This scenario leads to a collision of packets at node 1. Another scenario with four nodes within the range of each other is presented in Figure 6.5(b), where node 2 and node 3 both perform carrier sensing, find the medium is empty, and starts sending simultaneously. In this case, node 2 sends to node 1 , and at the same time node 3 sends to node 4 . This scenario also leads to a collision at the node 1 and 4 , since both of these nodes can overhear each other's transmission.

In the unsynchronized link state of the TR-MAC protocol, this problem of multiple simultaneous transmissions can cause a chain of collisions. If two transmitters using the default frequency offset find the medium is empty after performing clear channel assessment, then both of them start to send preambledata packet and wait for the acknowledgement. This preamble-data packet and acknowledgement listen iterations continues until an acknowledgement is received, or until the maximum network-wide known check interval value 


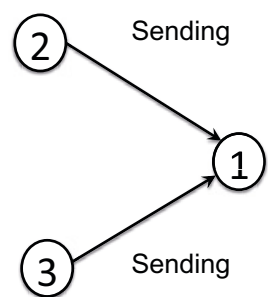

(a) Two senders sending to a single receiver

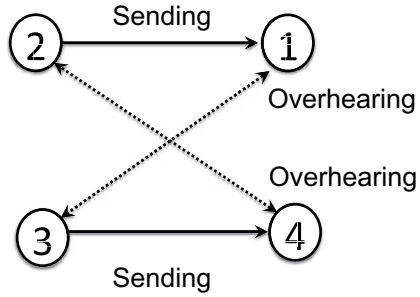

(b) Two senders sending to a two receivers

Figure 6.5: Multiple simultaneous transmissions

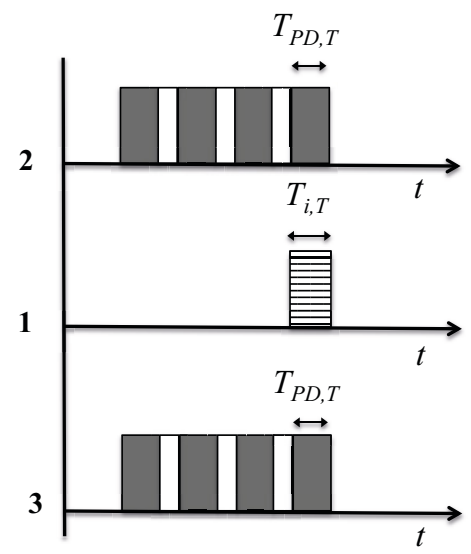

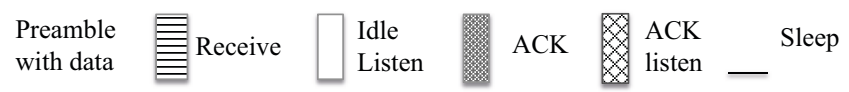

Figure 6.6: Multiple simultaneous transmissions with timeline

is reached. As a result, the preamble-data packets from two different senders experience collision at the desired receiver.

Figure 6.6 explains this situation from the perspective of two senders and one receiver, which elaborates the previously explained scenario of Figure 6.5(a). Here node 2 and 3 are the senders, and each of them sends simultaneously to 
node 1 using unsynchronized link state. We see that collision happens at node 1. As a result, node does not send any acknowledgement back to any of the sender nodes. To avoid this problem, one option is to add a random amount of preamble with the preamble-data packet to reserve the medium. This variation in the preamble size can solve this problem, since the transmitter that chooses the larger preamble can access the channel, where the other transmitter can backoff after receiving the preamble part of the preamble-data packet during its carrier sensing duration. This is an optional choice, by which the energy consumption for the transmitter increases with a small amount, as the size of a preamble is very small. The acknowledgement duration at the transmitter and the periodic listen duration at the receiver remain the same for the protocol after using this optional adjustment to avoid multiple simultaneous transmissions. WiseMAC uses a similar technique but in a different context. WiseMAC protocol uses a medium reservation preamble of random length before sending the actual short preamble to reserve the medium, when more than one transmitter node follow a single listen cycle of a receiver node in its synchronized communication[96]. The node that chooses the largest medium reservation preamble gets the access to the receiver for that particular cycle, because it was able to reserve the medium.

\subsubsection{Collision and retransmission}

A collision is costly from a protocol point of view, since a collision consumes energy for the sender nodes and the receiver node without a successful communication. Furthermore, the collided packets have to be retransmitted by all the participated sender nodes at a future time when the receiver node is active again. In the previous subsections, we have presented several techniques to minimize the probability of collisions for the TR-MAC protocol from the multiple access perspective to be operated in a WSN. Even though the collision probability can be minimized, a collision can still happen during the operation of a MAC protocol. Moreover, the frequency offset based transmit reference modulation has a limitation on the maximum number of simultaneous transmissions, which may result in packets being dropped. Hence, the upper MAC layer protocol has to manage the retransmission techniques. In this subsection, we discuss the 
collision and retransmission techniques for a dropped or collided packet in both unsynchronized and synchronized link states for the TR-MAC protocol.

During the unsynchronized link state of the TR-MAC protocol, one sender node performs carrier sense if it has a packet to send. If the sender finds the channel with the default frequency offset is empty and the interference level is not too high, then it transmits preamble-data packets for a maximum of the check interval duration or until an acknowledgement from the desired receiver is received. The check interval is defined as the maximum duration between two consecutive periodic listening times of a node. Since the sender node does not know the wake up time of the potential receiver node, this continuous packet sending by the sender node till the maximum check interval duration ensures that the potential receiver node can receive the packet whenever it wakes up to perform its periodic listening. If the sender node does not receive any acknowledgement after transmitting for a maximum of the check interval duration, it concludes that there was a problem in the communication. The cause of the problem can be that a collision with the preamble-data packets of another node prevented the preamble-data packet to be received by the receiver, or the receiver may have received the preamble-data packet, but the acknowledgement packet suffered a collision. Another potential problem can be that the potential receiver node is dead or out of range from the sender node. In all these cases, the sender node has to perform retransmission. The sender makes a retransmission attempt after a random multiple of the check interval duration. The sender does not perform immediate retransmission to avoid a repetition of the event that occurred during the previous check interval duration. After a number of failed retransmissions, the sender node concludes that the potential receiver node is dead or out of range from the sender node, hence discards the packet.

The sender node in the TR-MAC synchronized link state sends a packet to its synchronized and paired potential receiver using a previously agreed frequency offset and a time instance, if the channel was free during the preceding carrier sense duration. The sender node waits for the acknowledgement after sending the preamble-data packet. If the preamble-data packet is received correctly, then 
the receiver sends an acknowledgement back to the sender. The sender marks a successful communication after receiving the corresponding acknowledgement. If the sender node does not receive any acknowledgement from its paired receiver after a synchronized packet transfer, then the sender node marks an unsuccessful communication. The cause can be a collision of the preambledata packet, or the acknowledgement packet, or the synchronized node pair may be dead, out of range, or out of synchronization. In this context, out of synchronization refers to the scenario, where the one of the synchronized node in the pair may have changed its future wake up time instance or frequency offset, but the other node in the synchronized pair could not update to the new time instance or frequency offset. Loosing synchronization in this way between the synchronized pair nodes is not recommended. Therefore, the MAC protocol needs to ensure the that both the synchronized pair nodes updates to the new time or frequency offset, and maintain synchronization. In case of an unsuccessful communication by not receiving the acknowledgement packet from the synchronized receiver node, the sender node in the synchronized link state has to perform retransmission of the preamble-data packet by updating the future wake up time for that pair. The receiver node, on the other hand, assumes the preamble-data packet is lost if it does not receive any packet at the synchronized wake up time instance using the synchronized frequency offset. Hence, the receiver node wakes up again after updating its future wake up time for this paired sender node.

One design choice for the retransmission for synchronized link state can be that the synchronized sender pair concludes that the synchronized receiver pair is unreachable after a number of unsuccessful attempts, hence discards the packet. The drawback of this design choice is the synchronized sender pair concludes the receiver pair is unreachable too quickly, even though the receiver pair is still alive and within the range of the sender node. The reason for loosing the synchronization could be that both the synchronized pair was waking up to perform the sending and the receiving operation, however, the successful communication between the pair was prevented by the TR modulation's physical layer limitation of maximum number of simultaneous communication at a time 
instance using multiple frequency offsets.

Another design choice for the retransmission for synchronized link state could be to allow the synchronized pair nodes to fall back to the unsynchronized link state by assuming that the synchronization between the pair is lost, but that node is still alive and within the range of the sender node. Since the synchronized pair could not perform a successful communication using the previously agreed frequency offset and time instance, therefore, they fall back to the unsynchronized link state for future communications. After a successful communication in the unsynchronized link state, they again form a pair by moving to the synchronized link state by agreeing on a time instance and frequency offset for further communications. In the case of several unsuccessful communications in the unsynchronized link state, the sender node has no other option but to conclude that the desired receiver is dead or out of range from the receiver node. This design choice provides a more reliable communication strategy, because several unsuccessful communications in the synchronized link state leads to fall back to unsynchronized link state before finally declaring the node is unreachable. However, the drawback of this design choice is that the contention increases among nodes in the default frequency offset in the unsynchronized link state as more synchronized pair moves to the default frequency offset after loosing their synchronization. We recommend this design choice of falling back to unsynchronized link state before declaring a node is unreachable, because it adds reliability to the communication scheme of the protocol.

\subsubsection{State diagram from multiple access perspective}

We present a finite state diagram for the TR-MAC protocol operation for multiple access in Figure 6.7 with the possible states and events causing one node to change between its working states of sleeping, transmitting, and receiving. Each node also uses the state diagram for link state changing, provided in Section 6.1, Figure 6.2, to switch between the unsynchronized and the synchronized link states of the TR-MAC protocol.

After initializing in the INITIALIZATION state for the first time, one node 


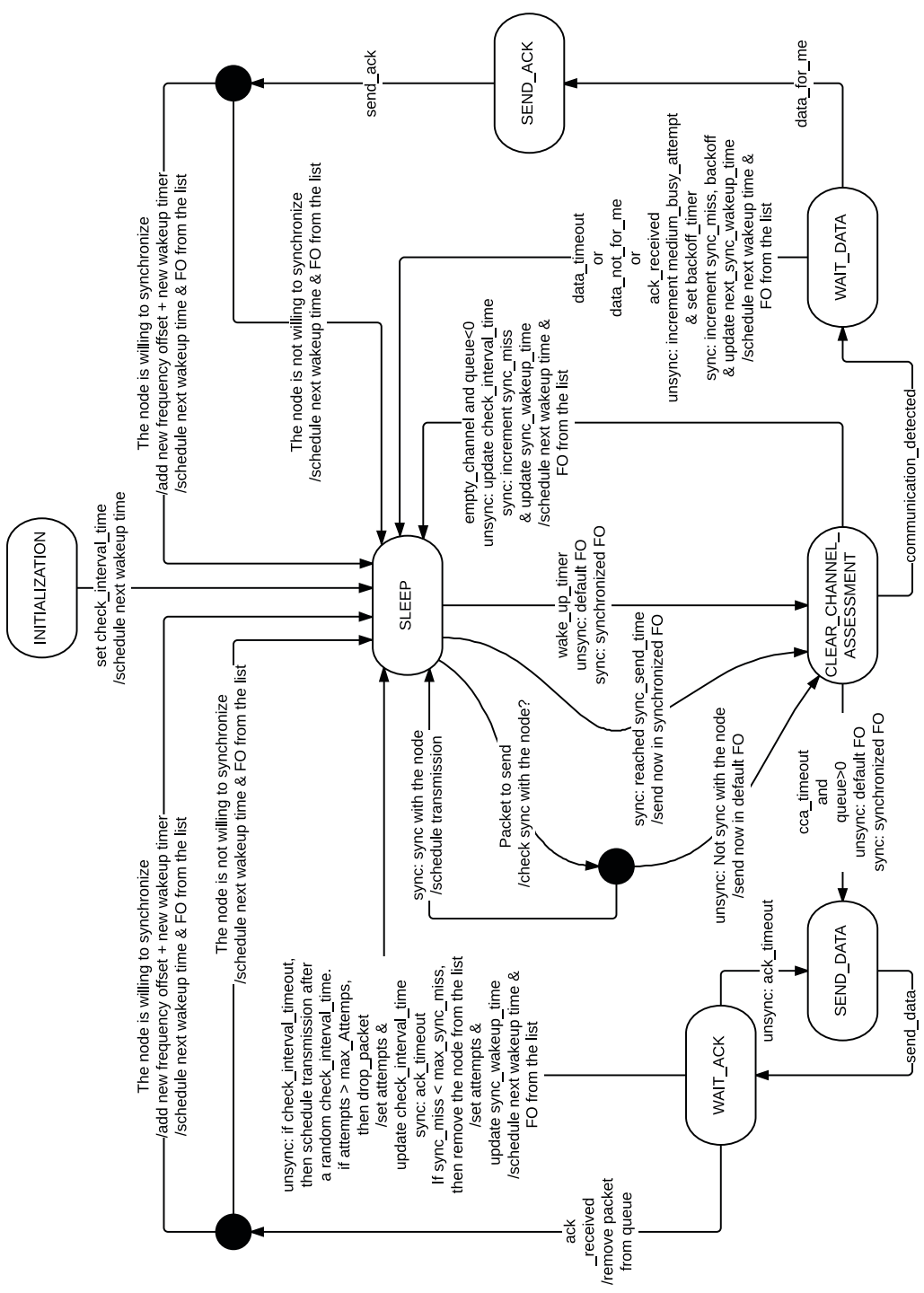

Figure 6.7: Finite state diagram for TR-MAC protocol operation 
starts operation in the unsynchronized link state and goes to SLEEP. One node chooses a periodic listening time, and wakes up to sense the channel for periodic listening duration in the default frequency offset by moving from the SLEEP state to the CLEAR_CHANNEL_ASSESSMENT state to perform carrier sense. If nothing was received during the carrier sense, and this node does not have any packet to send, then this node schedules its next periodic listening event after the network-wide known check interval duration and goes back to the SLEEP state. The node moves to the WAIT_DATA state if any communication was detected during the carrier sense in the CLEAR_CHANNEL_ASSESSMENT state. This node remains in the WAIT_DATA state until a complete preambledata packet is received. It discards the preamble-data if it was not destined for this node, or if any acknowledgement packet was received. Afterwards, this node schedules the next wakeup time and frequency offset from the list, and goes back to SLEEP. If the node was expecting a preamble-data packet from a synchronized pair at a synchronized frequency offset, and did not receive it; then it marks a sync_miss for that pair in the neighbor list. After a sync_miss, this node adds the next synchronized listen event in the event list before going to SLEEP. If the received preamble-data packet is destined for this node, then this node moves to the SEND_ACK state to send an acknowledgement back to the sender node from which it received the preamble-data packet. In this SEND_ACK state, this node decides to move to the synchronized link state for receiver-driven communication if the other node is willing to synchronize; or remain in the unsynchronized link state for this node if the other node is not willing to synchronize. If the other node is not willing to synchronize, then this node schedules the next wake up time and corresponding frequency offset, and goes to the SLEEP state. If this neighbor is willing to synchronize but does not exist in the neighbor list, then this node adds this neighbor in the neighbor list by allocating an unused frequency offset and non-overlapping time instance for that neighbor node to move to the synchronized link state with this neighbor. This synchronized listen event is also added to the event list. Afterwards, this current node sends the acknowledgement back to the sender neighbor node, schedules the next wake up event from the list, and goes to the SLEEP state. 
Next time this node wakes up to perform its periodic listening in the default frequency offset, or for an agreed time instance to receive or send a packet for a pairwise node in the synchronized link state in a synchronized frequency offset; depending on which comes first.

When one node receives a packet from the upper layer to send to a particular node, then it checks in the neighbor list whether there is already a synchronized link established with this particular neighbor node. Existence of the neighbor node in this node's the neighbor list means that a synchronized link has already been established with this neighbor including the future communication frequency offset and time instance. Therefore, it delays the packet transmission by scheduling a transmission to that agreed time instance, and goes back to SLEEP after scheduling the next event from the list. On the other hand, if the neighbor node is absent in this node's neighbor list, then this node realizes that it has to communicate in the unsynchronized link state with that particular node. Hence, this node moves to the CLEAR_CHANNEL_ASSESSMENT state to perform carrier sense in the default frequency offset from the SLEEP state. If the channel was busy during the carrier sensing, then the node moves to the WAIT_DATA state after detecting a communication. Afterwards, this node increments the medium_busy_attempt, backs off with a randomly chosen backoff timer, adds the next carrier sense event in the event list, schedules the next event from the event list, and goes back to the SLEEP state. If the channel was empty during the carrier sense, this node moves on to the SEND_DATA state to send the packet right away, then moves to the WAIT_ACK to wait for an acknowledgement from the receiver node. The node iterates in these two states of SEND_DATA and WAIT_ACK until it receives an acknowledgement from the desired receiver node or until the default check interval time is reached. After receiving the acknowledgement packet with all the information from the receiver node in the receiver-driven communication, this node decides about going to the synchronized link state using the state diagram for link state changing as mentioned in Figure 6.2 by checking the list about the proposed frequency offset and non-overlapping time instance. This node adds the information regarding the neighbor node in the neighbor list, if no overlapping time instance 
is present in the data structure. If an acknowledgement is missed, then this node schedules a retransmission event towards the receiver node in the event list. This node drops the packet after a maximum number of failing attempts in the unsynchronized state, when it is unable to receive an acknowledgement for several check interval durations from the intended receiver node. To perform backoff in the unsynchronized link state, the sender node schedules the next transmission after a random check interval duration in the event list. For the synchronized operation, this node sends a preamble-data packet, then waits for the acknowledgement. After a number of missed communications in the synchronized link state, this node removes the neighbor from the list, and falls back to the unsynchronized link state.

\subsection{Performance analysis}

In this section, we simulate the TR-MAC protocol to analyze its performance in a multiple access scenario. For simulation, we implement the TR-MAC protocol in $\mathrm{OMNeT}++$ simulator with MiXiM simulation framework [57]. OMNeT++ is a discrete event simulator to analyze a wireless protocol using events occurring at discrete time instances. An event can be a certain action in the protocol, for example, generating a packet following a certain distribution, or transmitting a packet at a certain time. The simulated protocol causes the node to change its states between sleeping, transmitting, or receiving at discrete point of time depending on the events. The impact of an event as the time progresses can be analyzed on the protocol level using the $\mathrm{OMNeT}++$ simulator. MiXiM is a simulation framework that uses the $\mathrm{OMNeT}++$ simulator for simulating wireless communication scenarios [57]. MiXiM uses protocol stacks for all the layers of OSI model. It also provides battery elements to compute energy consumption. Using this MiXiM simulation framework, we modelled the physical layer using the frequency offset based transmit reference system, and the MAC layer with our TR-MAC protocol, and used the default implementation for the rest of the layers to simulate a wireless sensor network. The individual battery parameters are configured in MiXiM together with the power levels. The default network 
layer is used, and the sensor application layer is configured with Poisson packet arrival with exponential inter-arrival times between packets.

In this section, at first we present the simulation setup in the physical layer with the frequency offset based transmit reference modulation as our underlying physical layer. Subsequently, we present the simulation setup for the TR-MAC protocol with its properties and provide parameter values. Afterwards, we present the results and performance analysis from the MAC layer perspective. We present the performance analysis for the synchronized link state establishment from the unsynchronized link state. We also present the impact of hidden terminal issue on the protocol. Here we also analyze the behavior of the protocol with the carrier sense and backoff mechanism. Finally, we present the system capacity and performance in terms of throughput and energy consumption for the system for a multiple access scenario.

\subsubsection{Simulation setup for the physical layer}

In this subsection, we present the physical layer setup in the simulation environment. The physical layer for using a specific modulation technique has impact on the sender side, at the channel, and on the receiver side. Thus, the effects at the sender, at the channel, and at the receiver have been taken into account to integrate the effects of using TR modulation in the physical layer in the simulator. The sender has to perform a mapping of transmission power and a mapping of the carrier frequency to transmit a signal to the channel. The Frii's free-space path loss model is used as the channel in the simulator, where the signal power of the transmitted signal gets attenuated based on the distance and the used carrier frequency [86]. The received signal power at the receiver is affected by the noise and interference of the channel. If this received power is greater than the receiver sensitivity, then the receiver decide that there is some valid signal to receive in the channel.

In the channel, the frequency offsets for the TR modulation are quite close to the carrier frequency of the reference signal. To ensure that the reference signal and the information signal are affected similarly by the channel, the maximum frequency offset has to be much smaller than the coherence band- 
width of the channel [15]. As a result, TR modulation experiences overlapping frequency spectrum. In comparison to a standard multichannel system, the TR modulation is highly affected by multi-user interference, where simultaneous communications using other frequency offsets act as the source of adjacent channel interference [87]. Furthermore, the noise floor at the receiver for the TR modulation is further enlarged by the self-mixing of the reference and the information signal [87]. Performance degradation further intensifies, if the spatial distribution of the nodes is such that the power received from an interfering node is much larger than the power received from the desired node. As a result, the feasible active interfering links are limited [17]. In the simulator, the receivers using the TR modulation analyze each received frame, and decide whether the received frame can be demodulated based on received SNIR by comparing it with a SNIR threshold.

\subsubsection{Simulation setup for the MAC layer}

In this subsection, we present the simulation setup for the MAC layer in the simulator. We have implemented the receiver-driven communication strategy, where one receiver allocates a frequency offset to the sender node after the first successful communication in the default frequency offset, and moves to the synchronized link state. In the scope of this thesis, we focus only on the receiver-driven communication strategy. Hence, the transmitter-driven strategy, and finally the energy-driven communication based on the available energy in a node are not considered within the scope of this thesis. Each node uses an event-based data structure to keep track of the events in order of their occurrence in time. The node also keeps track of its neighbors together with the communicating states. One node may communicate with its neighbor using the unsynchronized link state or synchronized link state. After establishing synchronized link state with a neighbor, one node has to keep track of the individual frequency offset, and the specific time instance to communicate with that particular neighbor pair. The TR-MAC protocol uses carrier sense with backoff mechanism. Hence, one node listens to the channel before sending a packet, and backs off if the channel was found busy. The TR-MAC protocol 
also provides retransmission mechanism in the presence of collision and loss of packets.

In Chapter 5, we have derived the number of usable frequency offsets for a given parameter setting, which was 26 frequency offsets. Among these total usable number of frequency offsets for our experimental setting, one frequency offset is assigned as the default frequency offset. Thus, one node can allocate at most 25 frequency offsets to its pairs to communicate in the synchronized link state. In order to avoid the communication of the specific time instance from the receiver to the sender pair, we bind the time offset with the frequency offset, as described in Subsection 6.1.3. Hence, the network-wide available maximum check interval duration is divided into 25 time instances, where each of them is bound with a particular frequency offset. For the TR-MAC protocol, we use the same parameter setting as mentioned in Table 1.2, in Chapter 3.

\subsubsection{Performance to establish the synchronized link}

In this subsection, we present the performance of the TR-MAC protocol in the very beginning phase to establish the synchronized link state with its neighbors. With this experiment, we want to analyze the operational insight of the common channel with the default frequency offset, which is used by a node in the network joining phase and neighbor discovery. In the beginning, the nodes start operation in the unsynchronized link state with the default frequency offset in the common channel. In the receiver-driven communication strategy, the receiver allocates a frequency offset to the sender node, and makes a pair with it after the first successful communication. The sender derives the time offset from the allocated frequency offset, and allocates a time instance by adding the time offset with its base check interval for sending towards that particular receiver node. For this experiment, the check interval is chosen as 1s. In this subsection, we provide performance analysis to establish the synchronized link state from the context of a single receiver, and multiple receivers by varying the number of active sender nodes and receiver nodes.

We consider a scenario with a single receiver node, where its neighbor sender nodes become active using a Poisson distribution. The maximum number of 


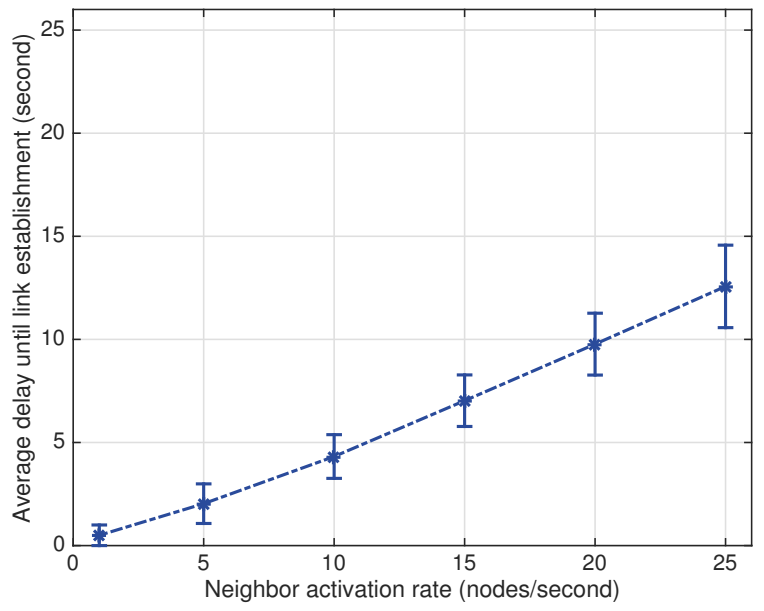

Figure 6.8: Performance to establish synchronized link for a single receiver

senders is chosen as 25 , since one receiver node can accommodate at most 25 sender nodes according to the scheme of binding the frequency offset together with the time instance. This binding avoids exchanging the time instance value in the header part of the preamble-data or the acknowledgement packet. The simulation stops when the receiver node has established synchronized link states with all the 25 sender nodes. We present the results for the synchronized link state establishment for a single receiver in Figure 6.8 in terms of the required time by varying the number of active neighbors in a second using a Poisson distribution.

In this figure, we observe that the average delay to establish a synchronized link is lower, when fewer neighbors are activated per second. As the number of active neighbors per second increases, the delay to establish the synchronized link increases. All the active sender nodes contend for the shared default frequency offset channel in the unsynchronized link state to send packets to the same receiver node. As the number of active neighbors increase, the contention increases. However, the sender node is offloaded from the default frequency 


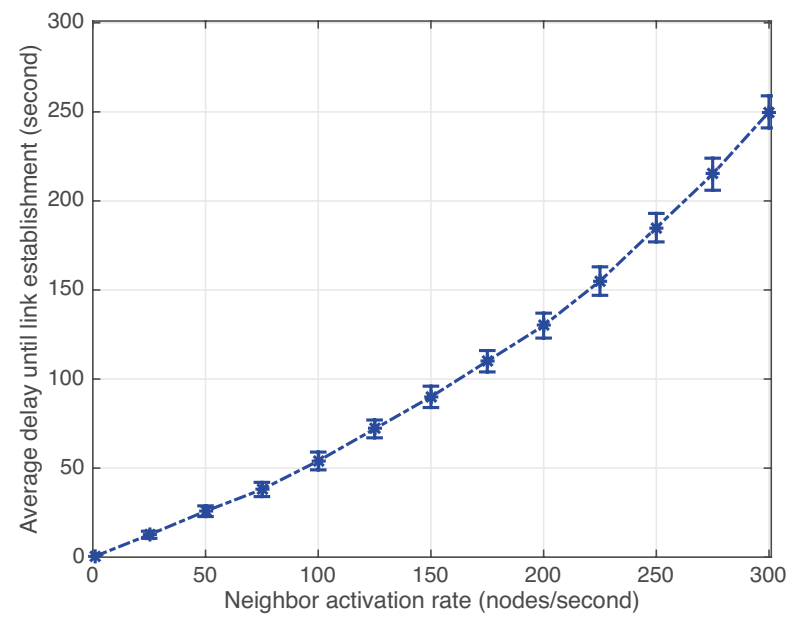

Figure 6.9: Performance of the default frequency offset to establish synchronized link for multiple receivers

channel to a different frequency offset channel after a successful communication using the unsynchronized link state. Thus, the other sender nodes do not have to contend with this sender node any more in the default frequency offset. The sender node that receives a packet from the upper layer, and starts sending first occupies the channel. The rest of the sender nodes perform clear channel assessment before sending a packet, and find the medium is busy. Hence, the rest of the sender nodes perform backoff, and try after that backoff time is over. The sender node, which occupied the channel, can send to the receiver node within $0.5 \mathrm{~s}$ for the best case scenario, which is half of the base check interval. From the figure, we see that on average $12.5 \mathrm{~s}$ delay is experienced to establish a synchronized link pairs for 25 sender nodes to a single receiver node. In this way, this experiments shows that all the sender pairs can establish synchronized links with a single receiver quite quickly, which is beneficial from the system level perspective.

Afterwards, we increase the number of nodes in the common channel to analyze 


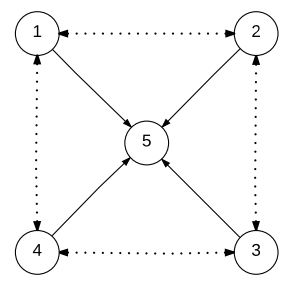

Figure 6.10: Hidden terminal setup

the capacity of this channel. For this simulation setup, each sender node tries to establish a synchronized link state with its intended receiver pair. Each sender node has a designated receiver node to which it sends the packets. All the active sender nodes contend with each other to access the channel to send packets to their respective intended receivers. Each of the sender nodes backs off if it finds the medium is already occupied. We stop the simulation when all the sender nodes have established synchronized links with their respective receiver pairs. Figure 6.9 represents the results in terms of average delay to establish the synchronized link state. Here we observe that the average delay remains around half of the neighbor activation rate with 125 active nodes, which is the desirable region at the system level. Afterwards, the average delay increases rapidly as the number of active nodes increases. This happens because the backoff timer was small for the nodes in the beginning. As one node experiences many backoffs, it increases its backoff timer to a relatively higher value for the next attempt. Therefore, the average delay reaches to a very high value for a higher number of active nodes. From this experiment, we realize that one sender link per receiver node scenario can have stable behavior till 125 links. In this way, we find an insight of the capacity limitation of the common channel with the default frequency offset from the system level perspective.

\subsubsection{Hidden terminal issue}

In this subsection, we analyze the behavior of the hidden terminal problem for our TR-MAC protocol to realize its effect in a multiple access scenario. 
Therefore, we perform a simple test for the hidden terminal problem with five nodes, where all of these nodes communicate in the unsynchronized link state using the default frequency offset. The simulation setup to analyze the hidden terminal problem is given in Figure 6.10.

In this simulation setup, four nodes from four corners send packets to the node in the middle using the default frequency offset. The central node 5 is in the range of each of the nodes in the corner, while the nodes in the opposite corner are out of range of each other. Hence, node 1 and 3 are out of range of each other, and node 2 and 4 are also out of range of each other. As a result, the nodes 1 and 3 are hidden to each other, as they cannot communicate directly to each other. Similarly, node 2 and 4 are hidden to each other. Hence, this simulation setup causes collisions in the central node 5 located in the middle position, because the hidden nodes are not aware of each other's transmissions towards node 5 . The central node 5 is active from the beginning. The four nodes in four corners are activated one after the other. We increase the number of active sources, and analyze the packet reception ratio and latency. The effect of the hidden terminal is analyzed in node 5 . The base check interval is considered as 1 second, and the traffic load is maintained in such a way that the activated nodes has packets to send for each of the check interval durations. When two nodes are made active, we always activate two nodes in the opposite corners. We use this setting to make represent a hidden terminal scenario. If the two nodes being active are in the range of each other, then they can listen to other's transmission and can backoff. For example, node 1 can overhear the transmission of node 2 , because they are in the range of each other.

We provide the packet reception ratio in Figure 6.11 by varying the number of source nodes. In this figure, we observe that the packet reception ratio is at its highest point when only one node is active. When 2 nodes are active, then we see a small drop in packet reception ratio. This drop in the packet reception ratio happens, because the two nodes being randomly active are the opposite ones in the corners, e.g., node 1 and 3, or node 2 and 4 . When three nodes are being active, then there is always one hidden node pair is present. Furthermore, one node backs off if it hears the communication of another node within its range. 


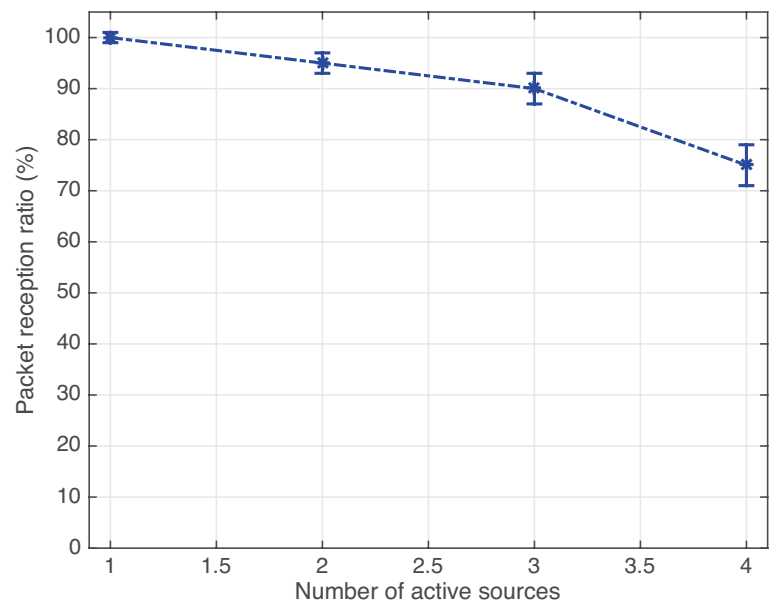

Figure 6.11: Packet reception ratio for the hidden terminal problem

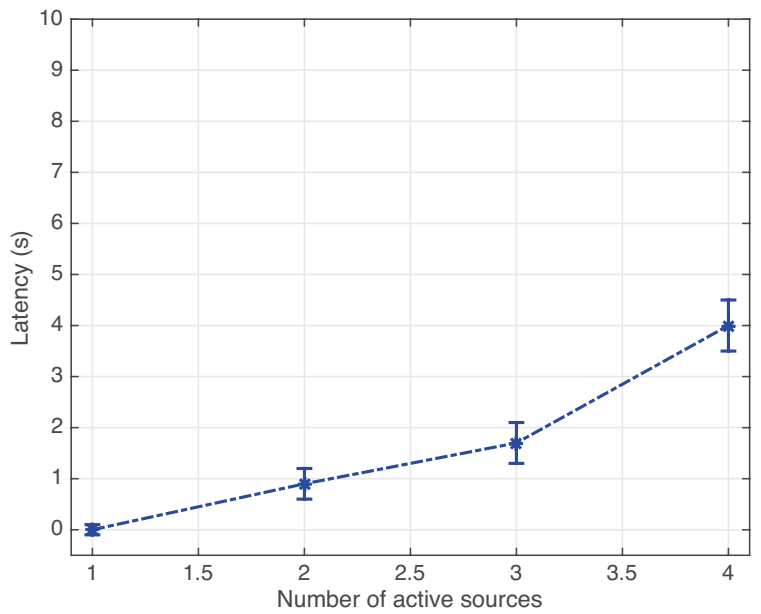

Figure 6.12: Latency for the hidden terminal problem 
For example, node 3 can overhear the communication of node 4 , and backs off. As a result, the packet reception ratio drops further. The lowest packet reception ratio can be seen with 4 active nodes, when two pairs of nodes act as hidden terminals to each other.

We present the latency results for the hidden terminal problem in Figure 6.12, where we observe a similar effect as the result with packet reception ratio. The latency is lower for a lower number of active nodes. The latency increases, as more nodes are being active, because of the hidden terminal problem. The latency is higher for 4 active nodes, because of two hidden terminal pairs.

All these tests for hidden terminal are done using the same frequency offset. In this case, we use the default frequency offset. If the communicating frequency offset is different, then the simultaneous communications with different frequency offsets do not interfere with each other. Based on these results of the hidden terminal issue, we can recommend to use as many different frequency offsets as possible for different transmission scenarios. The backoff mechanism of the protocol also prevents the collision using the same frequency offset, if one node can overhear the transmission of another node.

\subsubsection{System capacity and performance}

In this subsection, we analyze system capacity and performance for the TRMAC protocol with the underlying frequency offset based transmit reference modulation. Recall that the frequency offset based transmit reference modulation provides opportunity for multiple simultaneous transmissions using multiple frequency offsets. However, there exists a limitation on the number of simultaneous communications in the system, because of the increase in the multi-user interference in the presence of a large number of active links with multiple frequency offsets, and self-mixing of the reference and the information signals. We choose an area smaller than the maximum communication range, which means there is no hidden terminal problem if all the nodes are within this area. We use the number of active links as a parameter to analyze the system performance. We analyze the throughput and packet loss performance by varying the number of active links. We also present the energy-efficiency 


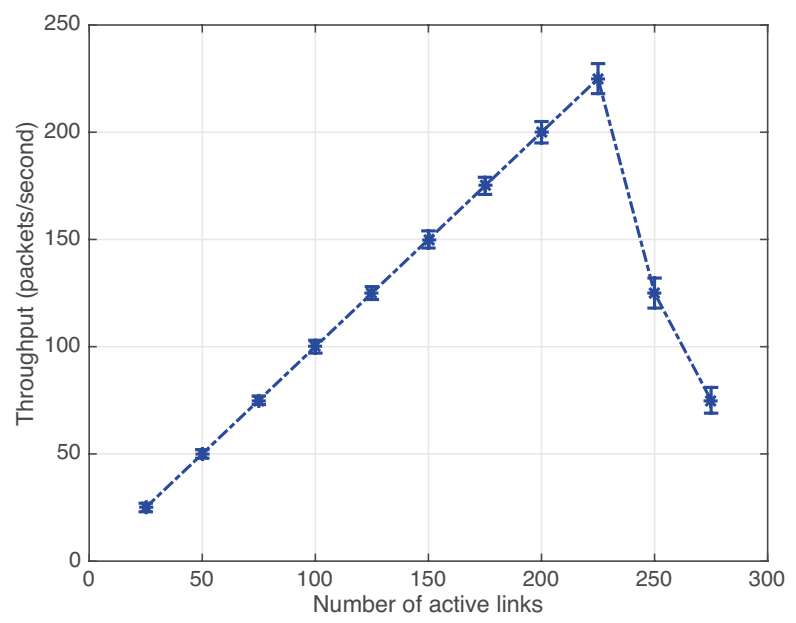

Figure 6.13: Throughput performance for varying number of active links

results for the same simulation setup. Finally, we present the throughput and packet loss performance by varying both the number of active links, and the number of links per receiver.

$i$. Throughput performance for varying number of active links: For this experiment, we use the receiver-driven communication strategy to setup synchronized links. One receiver node can have 25 synchronized pairs using 25 different frequency offsets. Thus, we use 25 nodes to send packets to the receiver nodes, and gradually increase the number of receivers to increase the number of active links. According to this simulation setup, increasing one receiver node increases the number of active links by 25. During the simulation duration, we make sure that the sender nodes have packets to send for all the check interval iterations to keep the links active by sending packets to the receiver node. The throughput result is presented in Figure 6.13 for varying number of active links. Here we observe that the throughput increases with the increasing number of active 
links until a certain range of active links, then the throughput experiences a sudden drop. The reason the throughput increases gradually with lower number of active links is that the receiver has full control of its sender pairs in the receiver-driven communication strategy to establish the synchronized links. The receiver node allocates the frequency offsets randomly to its sender pairs. As the receiver can allocate 25 sender pairs within each of its check interval duration, thus one receiver node can receive from 25 sender pairs. The time instances of communication with several sender pairs are derived from the allocated frequency offsets, as the time offset is bound with the frequency offset. As the periodic listen cycle of the receiver nodes are independent of each other, thus the time instances of communication with several sender pairs derived from the frequency offset are also independent of each other. For some cases the time instances of the communication using different frequency offset overlap, however the underlying transmit reference modulation allows multiple simultaneous communications using multiple frequency offsets to some extent. The authors in [17] analyzed the limitation on simultaneous communications for a desired receiver in the presence of several simultaneous transmissions using frequency offset based transmit reference modulation, and concluded at most 4 active transmissions within the acceptable interference level. As a result, increasing the number of receivers does not affect the throughput performance to that extent, since multiple communications mostly take place using different multiple frequency offsets.

The sudden drop in the throughput performance in Figure 6.13 is observed after 225 active links. This happens because of the limitation of maximum 4 active simultaneous communication links is reached after that number of active links. Whenever that happens, all the contributing simultaneous communication links at that time fail. As a result, we observe a high drop in the throughput value. We also calculate the system capacity by finding out how many non-overlapping data transmission instances with different frequency offset pairs can take place. Recall that the check interval of 1 s is divided into 25 time offsets to bind with 25 frequency offsets. Therefore, the time difference between two consecutive synchronized listen cycles is .038 seconds. The time duration to receive one com- 


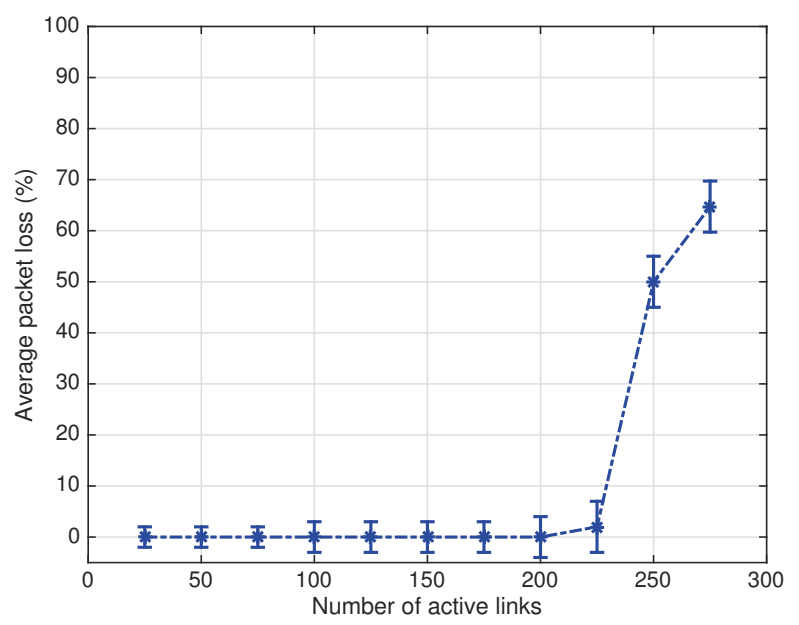

Figure 6.14: Packet loss performance for varying number of active links

plete preamble-data transmission includes one periodic listen duration, plus one acknowledgement duration; which is .00032 seconds according to the parameter setting in Table 1.2, in Chapter 3 considering $25 \mathrm{kbps}$ data rate. Therefore, dividing the time difference between two consecutive synchronized listen cycles (.038 seconds) by one successful packet reception duration (.00032 seconds) gives the maximum number of non-overlapping data transmission instances that can fit within two consecutive synchronized link cycles $(.038 / .00032=11.87)$. Afterwards, by multiplying the number of active links per receiver (25), together with the limitation for simultaneous communications (4), and with the amount of non-overlapping time instances (11.87), we can derive the total number of active links $(25 \times 4 \times 11.87=1187)$. From the result presented in Figure 6.13, we see the decrease of throughput performance after 225 active links, which is around 19\% when compared to the total number of available links $(225 / 1187) \times 100 \approx 19 \%)$. This 19\% efficiency result approximately corresponds to the maximum theoretical efficiency presented in Chapter 5 Section 5.4 using 4 simultaneous frequency offsets. 
ii. Packet loss performance for varying number of active links: We also analyze the packet loss results for the same simulation setup presented in Figure 6.14 follows the throughput results. Here we observe that there is no significant amount of packet loss until the number of active links reaches 225 links. Afterwards, the packet loss increases sharply, because of the overlapping in time for more than four simultaneous communications using multiple different frequency offsets destroys all the consecutive synchronized communication links of the involved receivers. Here packet loss means that the sender could not confirm whether the communication was successful. Receiving the acknowledgement packet in the sender marks a successful communication. A communication can be unsuccessful if the data packet was lost. For this case, the receiver does not send any acknowledgement towards the sender. An unsuccessful communication can also happen if the receiver node received the data packet, sent one acknowledgement towards the receiver, but the acknowledgement packet was lost. We considered a queue size of 10 to keep the generated packets in the sender node. A packet cannot be added to the queue at the sender node if the queue is full. A packet can also be dropped from the queue after a certain number of retransmission attempts if the intended receiver node is not reachable. However, the term packet loss in this context does not relate to the activity with the queue. Rather packet loss in this context refers to the fact that one communications was not successful. The retransmission attempts try to deliver the packet towards the intended receiver after an unsuccessful attempt.

iii. Energy-efficiency for varying number of active links: In this subsection, we analyze the energy consumption behavior of the system to analyze the energyefficiency of the system for the same simulation scenario. The energy-efficiency result is presented in Figure 6.15, where the mean power consumption per delivered packet is calculated by varying the number of active links. The total energy consumption during the whole simulation is divided by the total simulation time to derive the mean power consumption. Afterwards, this mean power consumption is divided by the total number of successfully delivered packet to obtain the value of the metric used to represent the energy-efficiency result. The behavior that we observe in the energy-efficiency figure is that the power 


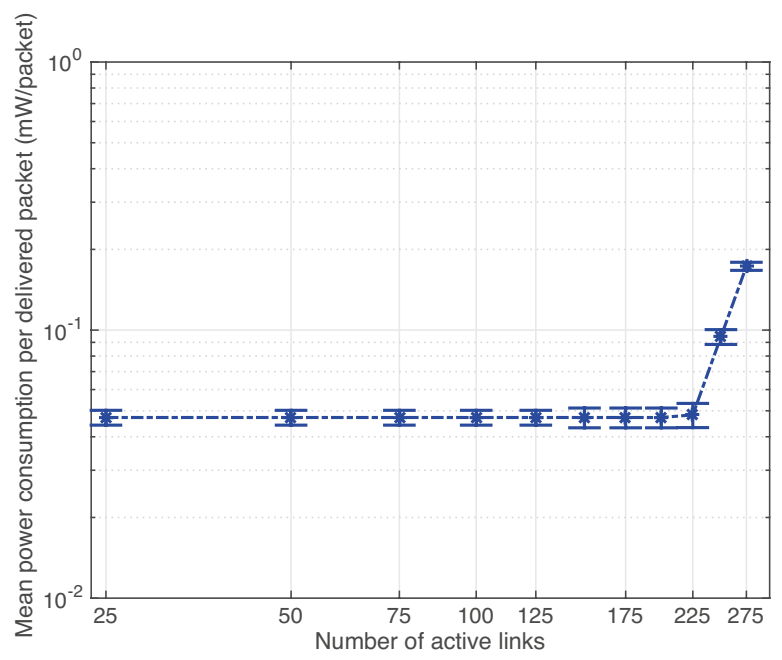

Figure 6.15: Energy-efficiency for varying number of active links

consumption remains stable with the increase of the throughput. Even though the throughput increase represents higher number of packet delivery, but this stability in the energy-efficiency result is obtained because the mean power consumption is divided by the number of successfully delivered packets. The mean power consumption per delivered packet increases when the throughput decreases. Although he energy consumption remains the same, but the decreasing throughput represents lower number of successful packet delivery. As a result, the mean power consumption per delivered packet increases after reaching the maximum throughput limit for the system. This energy-efficiency result ensures that the energy consumption for the TR-MAC protocol at the system level remains stable until the throughput limit of the system is reached. iv. Throughput performance for varying number of active links and varying number of links per receiver: We perform another experiment for the TR-MAC protocol by varying both the number of active links and the number of links per receiver. For the previous experiment, one receiver node was completely full with 25 sender pairs. As a result, the receiver was in full control of its sender pairs to allocate 


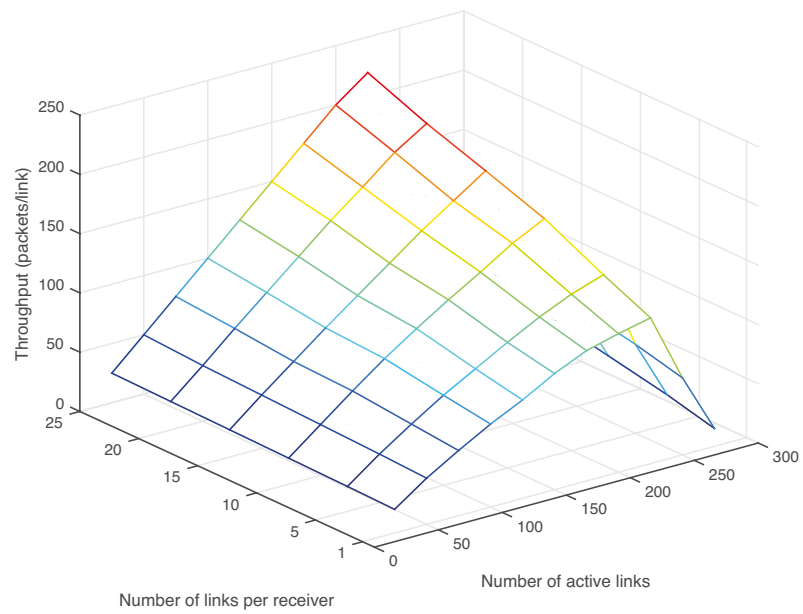

Figure 6.16: Throughput performance for varying number of active links and for varying number of links per receiver

non-overlapping time instances to them. For this experiment, we increase the number of nodes in an area to increase the number of links, but restrict the number of active links per receiver from 25 to 5 consecutively. The throughput result of this experiment is presented in Figure 6.16 in a three dimensional figure, where both the number of the active links and the number of the links per receiver are varying. Here we observe that the throughput increases for all the varying number of links per receiver until the maximum theoretical efficiency of 225 active links is reached for all the different number of links per receiver values, then the throughput sharply decreases. This behavior is similar to the behavior that we have observed earlier. We further observe that the throughput performance decreases as the number of links per receiver decreases for the same number of active links. The reason behind this behavior is that with many receiver nodes each having few numbers of synchronized links has a higher probability of having more than 4 simultaneous communications at the same time. Unlike the previous simulation setup, there are many receiver nodes 


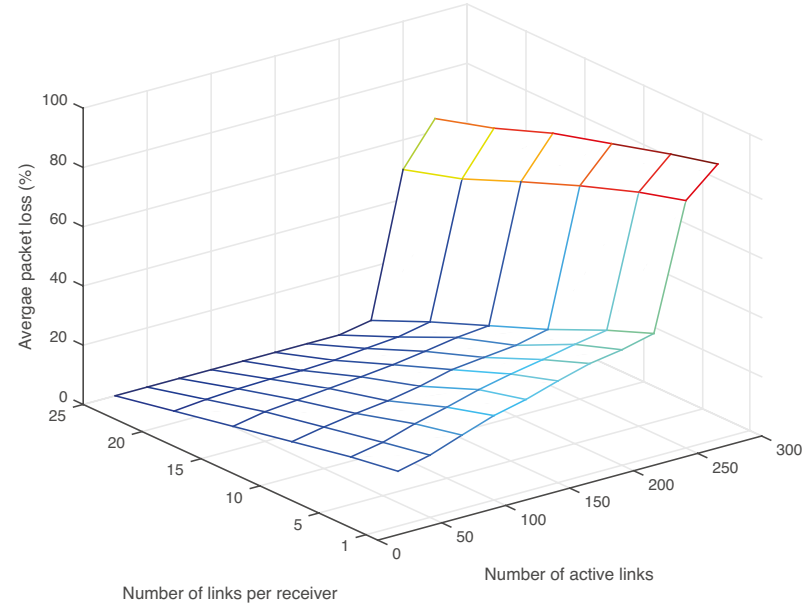

Figure 6.17: Packet loss performance for varying number of active links and for varying number of links per receiver

with few synchronized pairs. This contributes to the drop of the throughput for lower number of links per receiver for the same number of active links.

v. Throughput performance for varying number of active links and varying number of links per receiver: We present the packet loss results for the same simulation setup in Figure 6.17 by varying both the number of active links, and the number of links per receiver. We observe a similar expected behavior as the throughput results, where the packet loss for different number of links per receiver is slowing increasing until the maximum theoretical level of active links is reached. Afterwards, the packet loss increases sharply. We also observe that the packet loss also increases slightly as the number of links per receiver decreases for the same number of active links. The reason is that the increasing number of receivers with their few synchronized links increases the number of overlapping simultaneous communications at the same time.

From these experiments, we can derive the conclusion for the system level that higher number of links per receiver increases the throughput for the overall 
network. Although the number of links remains the same in network, but having lower number of links per receiver provides lower throughput. The reason behind this phenomenon is that when one receiver node has many pairs, then it can coordinate its pairs in different frequency offsets distributed over different time instances. On the other hand, the overlapping in time increases for the case where each of the receivers has fewer links. This overlapping in time for different frequency offsets limits the throughput, as the number of simultaneous communications becomes limited by the physical layer. The energy-efficiency for the system remains stable for the protocol operation with low data rate. However, the power consumption per delivered packet increases when the throughput decreases, because of the overlapping in time for simultaneous communications using different frequency offsets.

\subsection{Summary}

In this section, we present a detailed design of the TR-MAC protocol using frequency offset based transmit reference modulation. We further analyze the TR-MAC protocol performance for multiple access perspective, where multiple frequency offsets are used for multiple simultaneous communications. We propose two state diagrams for the TR-MAC protocol, one for the link state changing, and another one for the protocol operation for coordinating the receiving, transmitting, sleeping operations. We further discuss the impact of important multiple access criteria of carrier sense, hidden terminal problem, collisions, and retransmissions. Finally, we analyze the protocol performance for multiple access scenario and explain the maximum achievable system capacity and energy-efficiency based on the achievable throughput and packet loss results.

The medium access control mechanism for the TR-MAC protocol using the transmit reference modulation in the underlying physical layer has several strong points. The use of multiple frequency offsets for multiple simultaneous communications increases the overall system throughput. Furthermore, carrier sense with backoff mechanism reduces the collision, as one node listens to the 
channel before sending packets. The retransmission mechanism ensures that a packet is delivered to the receiver in the event of a data packet or an acknowledgement packet loss. Moreover, the energy consumption remains stable for low data rate operation scenario. However, the default frequency offset can become a bottleneck for the system, if higher number of nodes communicates using this offset. Although the MAC protocol provides energy-efficiency, but the inherent power penalty of the modulation technique in the physical layer makes this protocol not suitable for high data rate applications. The amount of frequency offsets provided by the physical layer can be a limiting factor for the system at the MAC layer. Although one frequency offset can be used in many different non-overlapping time instances, but a system with hundreds of nodes may require much higher frequency offsets. Moreover, the throughput of the system at the MAC layer becomes limited because of the limitation on the simultaneous communications of the physical layer.

Taking all these criteria into account, we recommend to use this TR-MAC protocol together with the frequency offset based transmit reference modulation for low data rate, and low duty cycle sparse WSN scenarios, for example, monitoring and tracking applications. 


\section{Conclusions and future work}

This thesis addresses energy-efficient medium access control protocols. Energyefficiency is an important requirement in the design of communication protocols for wireless sensor networks. The motivation for this thesis comes from the numerous applications of wireless sensor networks to create IoT, as well as, from the unique capabilities of the underlying frequency offset based transmit reference modulation technique. This thesis presents new insights about the communication problems of energy-constrained sensor nodes in a WSN by exploiting the opportunities, and at the same time minimizing the drawbacks provided by this underlying unique modulation technique. The ideas and solutions presented throughout the chapters of this thesis have the potential to address the requirements of modern IoT-based applications. The proposed solutions build up to a new communication protocol to use with transmit reference modulation to provide energy-efficient, traffic-adaptive, robust connectivity with multiple access control for the shared wireless medium.

This chapter is organized in the following manner: Section 7.1 presents our concluding remarks by elaborating our contributions in response to the research questions presented in Chapter 1. Afterwards, Section 7.2 presents possible future research directions to conclude this chapter. 


\subsection{Contributions and concluding remarks}

The thesis started with the aim to provide energy-efficient connectivity with multiple access control in the MAC layer for WSN customized for transmit reference modulation in the underlying physical layer. The main research question that we address in this thesis was:

How can the features of transmit reference modulation be exploited in a medium access control protocol for traffic-adaptive information dissemination with robust multiple access control in wireless sensor network, optimized for this radio technology in an energy-efficient manner?

In the following, we summarize the contributions of this thesis to answer the individual research questions presented in Chapter 1, which together answer the main research question mentioned above.

- Contribution 1: The design of an energy-efficient medium access control protocol using transmit reference modulation in the underlying physical layer.

With this contribution, we answer our first two research questions: research question 1: how can the features of transmit reference modulation be exploited in the MAC layer for WSNs?; and research question 2: how can transmit reference modulation be used to achieve energy-efficiency in WSNs?

In Chapter 3, we have presented a new energy-efficient MAC protocol for wireless sensor networks, called TR-MAC, which exploits the characteristics of transmit reference modulation in the MAC layer. The receiver architecture with TR modulation has the capability of fast signal acquisition and synchronization because of correlation based decoding. Thus a receiver using the TR-MAC protocol has the advantage of detecting a very small preamble. As a result, the transmitter using TR-MAC protocol appends small data packets ranging from very few bytes with the preamble, referred to as preamble-data. Furthermore, the frequency offset based TR modulation inherently provides a robust partitioning scheme by means of frequency offsets. The TR-MAC protocol takes this advantage and enables medium access control using different frequency offsets for multiple simultaneous communications. However, TR modulation has a 
power penalty in the transmitter because of sending the reference signal together with the information modulated signal. The TR-MAC protocol also minimizes this drawback by enabling energy-efficient protocol operation in the upper MAC layer.

We have provided a detailed mathematical model of the TR-MAC protocol operation from the perspective of energy consumption. During the initial unsynchronized link state operation of the preamble sampling based TRMAC protocol, one transmitter node transmits preamble-data, waits for the acknowledgement, and repeats this procedure until the maximum check interval duration, or until an acknowledgement is received from the desired receiver. We have derived an explicit mathematical expression for check interval optimization of the TR-MAC protocol, which will contribute to lower the energy consumption for the unsynchronized link operation.

The synchronized link state of the TR-MAC protocol enables a pair of nodes to communicate in an energy-efficient manner, where the transmitter node sends only one preamble-data at the previously agreed frequency offset and time instance towards its receiver pair. We have also proposed a mathematical model to optimize the protocol operation in the synchronized link state by waking the receiver a little earlier than the sender to ensure that only one iteration of preamble-data packet is enough for a successful communication in the presence of clock drift in the transmitter and the receiver nodes.

- Contribution 2: The design of traffic-adaptive duty cycle adaptation for periodic or event-driven communication patterns.

This contribution answers the research question 3: How can traffic-adaptive behavior be attained in WSNs while maintaining energy-efficiency? In Chapter 4, we have presented a traffic-adaptive duty cycle adaptation algorithm in order to provide responsiveness to traffic rate variations, which improves the protocol performance in the quality of service parameters while maintaining energy-efficiency. The traffic-adaptive duty cycle adaptation algorithm enables the receiver to increase its duty cycle based on incoming 
traffic rate. The corresponding sender node can follow this increasing duty cycle of the receiver node without any extra information exchange. Hence, this algorithm allows the protocol to adapt quickly to sudden higher traffic rate generated by event-driven scenarios.

Furthermore, we have combined our traffic-adaptive duty cycle adaptation algorithm together with request-based burst traffic transfer, where the later approach enables one transmitter node to send multiple packets from its queue destined to a single receiver node. This request-based approach achieves this benefit by using only a single bit to request the receiver to remain awake to receive multiple packets from the transmitter node after a successful communication. The consequence of preamble-data packet or acknowledgement packet losses on this traffic-adaptive algorithm has also been analyzed in Chapter 4 . All in all, this traffic-adaptive duty cycle adaptation combined with request-based burst packet transfer increases throughput and decreases packet delay while maintaining energy-efficiency when compared with other reference protocols with very limited information exchange.

- Contribution 3: The design of a model for multi-channel multiple access control using frequency offsets.

This contribution answers the research question 4: how does the multichannel properties affect the performance of multiple access control in a system using transmit reference modulation with frequency offsets? In Chapter 5 , we have presented a model to provide fundamental insight into the performance of a MAC protocol for transmit reference modulation with frequency offsets. The inherent capability of the underlying physical layer with transmit reference modulation enables the MAC layer to use multiple frequency offsets for multiple simultaneous communications. However, this excellent opportunity comes with certain limiting factors, e.g., the number of available frequency offsets is finite, and the total number of simultaneous transmitting frequency offsets has an upper limit. The limitation on the simultaneous transmission comes from the fact that 
many simultaneously active frequency offsets in the shared frequency spectrum increases the overall interference and noise floor. As a result, the receiver cannot decode the received signal, when the number of active transmissions using different frequency offsets crosses the limitation.

The model introduced in Chapter 5 is based on a basic multi-channel slotted-Aloha system, which uses a limited set of channels and a limit to the number of simultaneously used channels to represent the characteristics of the frequency offset based transmit reference modulation. This model investigates the opportunities and possibilities of frequency offset based TR modulation as a basic multiple access technique to analyze the potential benefits as well as to understand the consequences of the limiting factors at the upper MAC layer. We find that this model increases the throughput and efficiency for the MAC layer, which uses using frequency offset based TR modulation in the underlying physical layer.

- Contribution 4: The detailed design and performance analysis of the TR-MAC protocol from the multiple access perspective.

With this contribution, we answer the research question 5: how can an energy-efficient MAC protocol for WSNs be designed using transmit reference modulation for multi-channel medium access control using frequency offsets? In Chapter 6, we have provided a detailed design of the energy-efficient TR-MAC protocol for medium access control in WSNs from the multiple access perspective, which exploits the frequency offset based transmit reference modulation in the underlying physical layer. As part of the detailed design, we have introduced different communication strategies to establish the synchronized link state of the TR-MAC protocol from the unsynchronized link state, and a state-based mechanism for the link state changing procedure between these two states. For the multiple access analysis, we have considered the important criteria of carrier sense, exposed terminal issue, hidden terminal issue, collision and retransmission, etc. Furthermore, we have presented a finite state diagram for the TRMAC protocol operation in a multiple access scenario. Finally, we have 
presented the performance analysis of the TR-MAC protocol in a wireless sensor network with multiple access scenarios. The performance analysis shows that the MAC protocol can achieve higher throughput while maintaining energy-efficiency. The throughput increases because of the capability of using multiple frequency offsets for simultaneous transmissions provided by the underlying frequency offset based TR modulation.

The TR-MAC protocol is a novel variant of preamble sampling based protocol to exploit the fast synchronization capabilities of the frequency offset based transmit reference modulation. Energy-efficiency is achieved in this protocol by using a duty cycling mechanism and preamble sampling technique. Furthermore, the traffic-adaptive behavior provides adaptability to variable traffic rate. Moreover, this protocol also offers higher system capacity by exploiting the inherent multiple access capability of the transmit reference modulation that uses frequency offsets for multiple access control. Because of the inherent power and performance limitations of frequency offset based transmit reference modulation, the TR-MAC protocol is suitable for low data rate and low-duty-cycle sparse WSN scenarios, for example, monitoring and tracking applications.

\subsection{Future work}

This thesis presents a customized energy-efficient MAC protocol to exploit the specific features of TR modulation in the underlying physical layer with robust and traffic-adaptive communication including multiple access capability by using frequency offsets. However, there are still open areas to be addressed in future work, which are discussed below:

- Energy-driven communication: The proposed TR-MAC protocol enjoys tremendous flexibility because of the options to communicate in either receiver-driven or transmitter-driven strategy for both the link layer, and for multi-hop communication. For example, one node having less energy can transfer the energy consumption burden by requesting another node to follow its lead. Other nodes can follow the lead of the energy-limited 
node by remembering the next communication time of this energy-limited node. In this way, the protocol can effectively operate like an energydriven protocol, where the protocol operation takes place based on the available energy of a node. The transmitter-driven communication strategy can be investigated further for efficient broadcasting, where one node makes its first-hop neighbors to follow its lead.

- Energy harvesting: To extend the network lifetime by minimizing energy consumption is one of main challenges in the sensor network research domain. One way to increase the battery lifetime of the wireless sensor node is to use energy harvesting. The sources of energy harvesting can be from the environment, e.g., solar energy; or from other energy sources, e.g., motion, pressure, or heat. The energy availability may be unevenly distributed between nodes, which have to be taken into account when using energy harvesting. For instance, all the nodes in a network may not be equally exposed to sunlight in case of energy harvesting from solar energy. Protocol operation must take this behavior into account.

- Traffic-adaptive behavior with multiple links: The traffic-adaptive algorithm presented in this thesis to disseminate increased amount of packets generated by event-driven scenario is evaluated for a single-hop network operation. As a future work, this traffic-adaptive algorithm can be evaluated for multi-hop network operation using multiple link scenarios.

- Effect of using multi-channel communication using frequency offsets at upper layers: The TR-MAC protocol together with the underlying frequency offset based transmit reference modulation provides multi-channel-like behavior, since it allows medium access control by using different frequency offsets simultaneously. As a result, a single receiver can offer performance like in a multi-channel system with limited complexity, where the frequency offsets provide the inherent channel partitioning. The opportunities that this multi-channel communication using frequency offsets provides to the upper layer can be researched further. 
- Energy-efficient multi-hop communication: In this thesis, we evaluated low duty cycle energy-efficient TR-MAC protocol for single-hop network operation. We also considered a flat topology, where all the nodes are equal. At the network layer, further research can be done to evaluate this protocol for extremely low duty cycle network operation in a multi-hop environment using a clustered topology in designing simple routing algorithms.

- Impact of the physical layer features in the upper MAC layer: In this thesis, the TR-MAC protocol is evaluated with a simplified version of the physical layer with the frequency offset based transmit reference modulation. The physical layer model can be improved by considering channel fading, and by considering the near-far effect with difference distances for multiple transmitter nodes from a single receiver node. Furthermore, the receiver architecture with the exact demodulation technique in the physical layer can be extended to represent the operation of the modulation technique at the receiver.

- Combination of MAC Protocol, physical layer and IC-design: The MAC protocol deals with the low duty cycle and robust communication aspects of the wireless sensor network. The underlying physical layer focuses on the modulation in the transmitter, channel models, and the demodulation in the receiver. The IC-design aims to realize the extremely low power implementation of these concepts. As a result, future work on the realization of a sensor node might include the combination of low-power IC design for the radio transceiver, the frequency offset based transmit reference modulation in the physical layer, and finally, the customized TR-MAC protocol as a communication protocol optimized for this radio technology. 


\section{Bibliography}

[1] I. F. Akyildiz, W. Su, Y. Sankarasubramaniam, and E. Cayirci, “A survey on sensor networks," IEEE Communications Magazine, vol. 40, no. 8, pp. 102-114, Aug. 2002.

[2] P. Huang, L. Xiao, S. Soltani, M. W. Mutka, and N. Xi, “The evolution of MAC protocols in wireless sensor networks: A survey." IEEE Communications Surveys and Tutorials, vol. 15, no. 1, pp. 101-120, 2013.

[3] K.G.Langendoen, "Medium access control in wireless sensor networks," in Medium Access Control in Wireless Networks, H. Wu and Y. Pan, Eds. Nova Science Publishers, Inc., May 2008, pp. 535-560.

[4] S. Sudevalayam and P. Kulkarni, "Energy harvesting sensor nodes: Survey and implications." IEEE Communications Surveys and Tutorials, vol. 13, no. 3, pp. 443-461, 2011.

[5] A. Bachir, M. Dohler, T. Watteyne, and K. K. Leung, "MAC essentials for wireless sensor networks." IEEE Communications Surveys and Tutorials, vol. 12, no. 2, pp. 222-248, 2010.

[6] C. Cano, B. Bellalta, A. Sfairopoulou, and M. Oliver, "Low energy operation in WSNs: A survey of preamble sampling MAC protocols," Computer Networks, vol. 55, no. 15, pp. 3351-3363, Oct. 2011.

[7] J. Haartsen, X. Shang, J. Balkema, A. Meijerink, and J. Tauritz, “A new wireless modulation technique based on frequency-offset," in Proceedings of the 12th Symposium on Communications and Vehicular Technology in the Benelux. Enschede, The Netherlands: Twente University Press, 2005.

[8] A. Meijerink, S. Cotton, M. Bentum, and W. Scanlon, "Noise-based frequency offset modulation in wideband frequency-selective fading channels," in 16th Annual Symposium of the IEEE/CVT. Louvain-la-Neuve, Belgium: IEEE/SCVT, 2009.

[9] I. F. Akyildiz and M. C. Vuran, Wireless Sensor Networks. Wiley, 2010. 
[10] R. Hoctor and H. Tomlinson, "Delay-hopped transmitted-reference RF communications," in IEEE Conference on Ultra Wideband Systems and Technologies, 2002, pp. 265-269.

[11] J. D. Choi and W. E. Stark, "Performance of ultra-wideband communications with suboptimal receivers in multipath channels." IEEE Journal on Selected Areas in Communications, vol. 20, no. 9, pp. 1754-1766, 2002.

[12] J. Haartsen, A. Meijerink, A. Bekkaoui, A. Taban, and J. Tauritz, “Novel wireless modulation technique based on noise," in 11th Symposium on Communications and Vehicular Technology in the Benelux (SCVT 2004), Gent, Belgium, 2004.

[13] D. Goeckel and Q. Zhang, "Slightly frequency-shifted reference ultra-wideband (UWB) radio." IEEE Transactions on Communications, vol. 55, no. 3, pp. 508-519, 2007.

[14] Z. Lai, H. Joshi, D. Goeckel, D. Gupta, D. Gupta, and A. Mathew, "Performance of UWB systems in the presence of severe multipath and narrowband interference," in Ultra-Wideband, 2008. ICUWB 2008. IEEE International Conference on, vol. 1, Sept 2008, pp. 85-88.

[15] I. Bilal, A. Meijerink, and M. J. Bentum, “Performance analysis of noise-based frequency offset modulation in dense frequency-selective fading channels," in 9th International Conference on Signal Processing and Communication Systems, ICSPCS 2015, Cairns, Australia, December 14-16, 2015, 2015, pp. 1-7.

[16] I. Bilal, A. Meijerink, and M. Bentum, "Single-tone interference in noise-based frequency offset modulation," in Communications and Vehicular Technology in the Benelux (SCVT), 2014 IEEE 21st Symposium on, Nov 2014, pp. 24-29.

[17] B. I. Bitachon, I. Bilal, A. Meijerink, and M. J. Bentum, “Near-far problem in noisebased frequency-offset modulation," in Communications and Vehicular Technology in the Benelux (SCVT), 2015 IEEE Symposium on, Nov 2015, pp. 1-6.

[18] WALNUT Project, Std., 2012. [Online]. Available: http://www.utwente.nl/ctit/ research/research_projects/national/stw/walnut

[19] S. Morshed and G. J. Heijenk, "TR-MAC: an energy-efficient MAC protocol for wireless sensor networks exploiting noise-based transmitted reference modulation," in 2nd Joint ERCIM eMobility and MobiSense Workshop, St. Petersburg, Russia, June 2013, pp. 58-71.

[20] S. Morshed and G. Heijenk, "TR-MAC: An energy-efficient MAC protocol exploiting transmitted reference modulation for wireless sensor networks," in Proceedings of 
the 17th ACM International Conference on Modeling, Analysis and Simulation of Wireless and Mobile Systems, ser. MSWiM '14. ACM, 2014, pp. 21-29.

[21] S. Morshed and G. J. Heijenk, “Optimization and verification of the TR-MAC protocol for wireless sensor networks." in WWIC, ser. Lecture Notes in Computer Science, vol. 9071. Springer, 2015, pp. 396-410.

[22] S. Morshed, M. Baratchi, and G. Heijenk, "Traffic-adaptive duty cycle adaptation in TR-MAC protocol for wireless sensor networks," in 2016 Wireless Days (WD), March 2016, pp. 1-6.

[23] S. Morshed, M. Baratchi, P. K. Mandal, and G. Heijenk, "A multi-channel multiple access scheme using frequency offsets - modelling and analysis," in 2016 WiMob, October 2016, pp. 1-6.

[24] W. Ye, J. Heidemann, and D. Estrin, "An energy-efficient mac protocol for wireless sensor networks," in INFOCOM 2002. Twenty-First Annual Joint Conference of the IEEE Computer and Communications Societies. Proceedings. IEEE, vol. 3, 2002, pp. 1567-1576 vol.3.

[25] P. Hurni and T. Braun, "MaxMAC: A maximally traffic-adaptive MAC protocol for wireless sensor networks," in Wireless Sensor Networks, 7th European Conference, EWSN 2010, Coimbra, Portugal, February 17-19, 2010. Proceedings, 2010, pp. 289-305.

[26] O. D. Incel, "Survey paper: A survey on multi-channel communication in wireless sensor networks," Computer Networks, vol. 55, no. 13, pp. 3081-3099, Sep. 2011.

[27] I. Demirkol, C. Ersoy, and F. Alagoz, "MAC protocols for wireless sensor networks: a survey," Communications Magazine, IEEE, vol. 44, no. 4, pp. 115-121, April 2006.

[28] K. Kredo II and P. Mohapatra, "Medium access control in wireless sensor networks," Computer Networks, vol. 51, no. 4, pp. 961-994, Mar. 2007.

[29] J. Yick, B. Mukherjee, and D. Ghosal, "Wireless sensor network survey," Computer Networks, vol. 52, no. 12, pp. 2292-2330, Aug. 2008.

[30] G. P. Halkes and K. Langendoen, "Energy-efficient medium access control." in Embedded Systems Handbook, R. Zurawski, Ed. CRC Press, 2005.

[31] S. C. Ergen and P. Varaiya, "PEDAMACS: Power efficient and delay aware medium access protocol for sensor networks." IEEE Transactions on Mobile Computing, vol. 5, no. 7, pp. 920-930, 2006. 
[32] L. van Hoesel and P. Havinga, "A lightweight medium access protocol (LMAC) for wireless sensor networks: Reducing preamble transmissions and transceiver state switches," in 1st International Workshop on Networked Sensing Systems, INSS 2004. Tokio, Japan: Society of Instrument and Control Engineers (SICE), 2004, pp. 205-208.

[33] V. Rajendran, K. Obraczka, and J. J. Garcia-Luna-Aceves, “Energy-efficient, collisionfree medium access control for wireless sensor networks," Wireless Networks, vol. 12, no. 1, pp. 63-78, Feb. 2006.

[34] G. P. Halkes and K. G. Langendoen, "Crankshaft: An energy-efficient mac-protocol for dense wireless sensor networks," in Proceedings of the 4th European Conference on Wireless Sensor Networks, ser. EWSN'07. Berlin, Heidelberg: Springer-Verlag, 2007, pp. 228-244.

[35] W. Ye, J. S. Heidemann, and D. Estrin, "Medium access control with coordinated adaptive sleeping for wireless sensor networks." IEEE/ACM Transaction on Networking, vol. 12, pp. 493-506, 2004.

[36] T. van Dam and K. Langendoen, "An adaptive energy-efficient MAC protocol for wireless sensor networks." in SenSys. ACM, 2003, pp. 171-180.

[37] S. Du, A. K. Saha, and D. B. Johnson, "RMAC: A routing-enhanced duty-cycle mac protocol for wireless sensor networks," in IEEE INFOCOM 2007 - 26th IEEE International Conference on Computer Communications, May 2007, pp. 1478-1486.

[38] J. Polastre, J. Hill, and D. E. Culler, "Versatile low power media access for wireless sensor networks." in SynSys, 2004, pp. 95-107.

[39] M. Buettner, G. V. Yee, E. Anderson, and R. Han, "X-MAC: A short preamble MAC protocol for duty-cycled wireless sensor networks," in Proceedings of the 4th International Conference on Embedded Networked Sensor Systems, ser. SenSys '06. ACM, 2006, pp. 307-320.

[40] A. El-Hoiydi and J.-D. Decotignie, “WiseMAC: An ultra low power MAC protocol for multi-hop wireless sensor networks." in ALGOSENSORS, ser. Lecture Notes in Computer Science, vol. 3121. Springer, 2004, pp. 18-31.

[41] G.-S. Ahn, S. G. Hong, E. Miluzzo, A. T. Campbell, and F. Cuomo, "FunnelingMAC: A localized, sink-oriented MAC for boosting fidelity in sensor networks," in Proceedings of the 4th International Conference on Embedded Networked Sensor Systems, ser. SenSys '06. New York, NY, USA: ACM, 2006, pp. 293-306. 
[42] I. Rhee, A. Warrier, M. Aia, J. Min, and M. L. Sichitiu, "Z-MAC: a hybrid MAC for wireless sensor networks." IEEE/ACM Transaction on Networking, vol. 16, pp. 511-524, 2008.

[43] W. Ye, F. Silva, and J. Heidemann, "Ultra-low duty cycle MAC with scheduled channel polling," in Proceedings of the 4th International Conference on Embedded Networked Sensor Systems, ser. SenSys '06. New York, NY, USA: ACM, 2006, pp. 321-334.

[44] ZigBee Alliance, Std., June 2017. [Online]. Available: http:/ /www.zigbee.org

[45] C. Schurgers, V. Tsiatsis, S. Ganeriwal, and M. B. Srivastava, “Optimizing sensor networks in the energy-latency-density design space." IEEE Transactions on Mobile Computing, vol. 1, no. 1, pp. 70-80, 2002.

[46] M. Avvenuti, P. Corsini, P. Masci, and A. Vecchio, "Increasing the efficiency of preamble sampling protocols for wireless sensor networks," in Mobile Computing and Wireless Communication International Conference, 2006. MCWC 2006. Proceedings of the First, Sept 2006, pp. 117-122.

[47] K.-J. Wong and D. K. Arvind, "SpeckMAC: Low-power decentralised MAC protocols for low data rate transmissions in specknets," in Proceedings of the 2 nd International Workshop on Multi-hop Ad Hoc Networks: From Theory to Reality, ser. REALMAN '06. New York, NY, USA: ACM, 2006, pp. 71-78.

[48] H. Wang, X. Zhang, F. Naït-Abdesselam, and A. A. Khokhar, “DPS-MAC: An asynchronous MAC protocol for wireless sensor networks." in HiPC, ser. Lecture Notes in Computer Science, S. Aluru, M. Parashar, R. Badrinath, and V. K. Prasanna, Eds., vol. 4873. Springer, 2007, pp. 393-404.

[49] K. Han, S. Lim, S. Lee, J. W. Lee, and S. An, "Signaling-embedded short preamble mac for multihop wireless sensor networks." in ICOIN, ser. Lecture Notes in Computer Science, T. M. Vazao, M. M. Freire, and I. Chong, Eds., vol. 5200. Springer, 2007, pp. 1-10.

[50] S. Mahlknecht and M. Bock, "CSMA-MPS: a minimum preamble sampling MAC protocol for low power wireless sensor networks," in Factory Communication Systems, 2004. Proceedings. 2004 IEEE International Workshop on, 2004, pp. 73-80.

[51] A. Dunkels, "The ContikiMAC Radio Duty Cycling Protocol," Swedish Institute of Computer Science, Tech. Rep. T2011:13, Dec. 2011.

[52] P. Kumar, M. Günes, Q. Mushtaq, and B. Blywis, "A real-time and energy-efficient MAC protocol for wireless sensor networks," in 6th IEEE International Conference on Wireless and Optical Communications Networks, Cairo, Egypt, April 2009. 
[53] E.-y. Lin, J. M. Rabaey, and A. Wolisz, "Power-efficient rendez-vous schemes for dense wireless sensor networks," in Proc. of ICC 2004 Paris, June 2004, ConferenceProceedings.

[54] X. Shi and G. Stromberg, "SyncWUF: An ultra low-power MAC protocol for wireless sensor networks." IEEE Transactions on Mobile Computing, vol. 6, pp. 115-125, 2007.

[55] X. Zhang, J. Ansari, and P. Mähönen, “Traffic aware medium access control protocol for wireless sensor networks," in Proceedings of the 7th ACM International Symposium on Mobility Management and Wireless Access, ser. MobiWAC '09. New York, NY, USA: ACM, 2009, pp. 140-148.

[56] C. J. Merlin and W. B. Heinzelman, "Schedule adaptation of low-power-listening protocols for wireless sensor networks." IEEE Transactions on Mobile Computing, vol. 9, no. 5, pp. 672-685, 2010.

[57] P. Hurni, T. Braun, and M. Anwander, "Evaluation of WiseMAC and extensions on wireless sensor nodes." Telecommunication Systems, vol. 43, no. 1-2, pp. 49-58, 2010.

[58] M. Anwander, G. Wagenknecht, T. Braun, and K. Dolfus, “BEAM: A burst-aware energy-efficient adaptive MAC protocol for wireless sensor networks," in Seventh International Conference on Networked Sensing Systems (INSS), June 2010, pp. 195 -202.

[59] M. Avvenuti and A. Vecchio, "Adaptability in the B-MAC+ protocol." in ISPA. IEEE Computer Society, 2008, pp. 946-951.

[60] R. Jurdak, P. Baldi, and C. V. Lopes, "Energy aware low power listening for sensor networks," in Second International Workshop on Networked Sensing Systems (INSS05), 2005.

[61] E. R. Sanchez, C. Chaudet, and M. Rebaudengo, "Improving preamble sampling performance in wireless sensor networks with state information," in Proceedings of the Sixth International Conference on Wireless On-Demand Network Systems and Services, ser. WONS'09. Piscataway, NJ, USA: IEEE Press, 2009, pp. 93-100.

[62] Y. Wu, S. Fahmy, and N. B. Shroff, "Optimal sleep/wake scheduling for timesynchronized sensor networks with QoS guarantees." IEEE/ACM Transactions on Networking, vol. 17, no. 5, pp. 1508-1521, 2009.

[63] A. El-hoiydi, J.-D. Decotignie, and J. Hernandez, "Low power MAC protocols for infrastructure wireless sensor networks," in In Proceedings of the Fifth European Wireless Conference, 2004, pp. 563-569. 
[64] A. El-Hoiydi and J.-D. Decotignie, “WiseMAC: An ultra low power mac protocol for the downlink of infrastructure wireless sensor networks," in Proceedings of the Ninth International Symposium on Computers and Communications, ser. ISCC '04. IEEE Computer Society, 2004, pp. 244-251.

[65] A. El-hoiydi and J.-D. Decotignie, "Low power downlink MAC protocols for infrastructure wireless sensor networks," Mobile Networks and Applications, vol. 10, no. 5, pp. 675-690, Oct 2005.

[66] A. Köpke, M. Swigulski, K. Wessel, D. Willkomm, P. T. K. Haneveld, T. E. V. Parker, O. W. Visser, H. S. Lichte, and S. Valentin, "Simulating wireless and mobile networks in OMNeT++ the MiXiM vision," in Proceedings of the 1st International Conference on Simulation Tools and Techniques for Communications, Networks and Systems $\mathcal{E}$ Workshops, ser. Simutools '08. ICST, Brussels, Belgium, Belgium: ICST (Institute for Computer Sciences, Social-Informatics and Telecommunications Engineering), 2008, pp. 71:1$71: 8$.

[67] M. Doudou, D. Djenouri, and N. Badache, "Survey on latency issues of asynchronous MAC protocols in delay-sensitive wireless sensor networks," IEEE Communications Surveys Tutorials, vol. 15, no. 2, pp. 528-550, 2013.

[68] K. Stone and M. Colagrosso, "Efficient duty cycling through prediction and sampling in wireless sensor networks," Wireless Communications and Mobile Computing, vol. 7, no. 9, pp. 1087-1102, 2007.

[69] C. Cano, B. Bellalta, A. Sfairopoulou, and J. Barcelo, “A low power listening MAC with scheduled wake up after transmissions for wsns." IEEE Communications Letters, vol. 13, no. 4, pp. 221-223, 2009.

[70] C. J. Merlin and W. B. Heinzelman, "Duty cycle control for low-power-listening MAC protocols," IEEE Transactions on Mobile Computing, vol. 9, no. 11, pp. 1508-1521, Nov 2010.

[71] M. Doudou, D. Djenouri, J. M. Barcelo-Ordinas, and N. Badache, “Delay-efficient MAC protocol with traffic differentiation and run-time parameter adaptation for energy-constrained wireless sensor networks," Wireless Networks, vol. 22, no. 2, pp. 467-490, 2016.

[72] L. Wu, S. Zhuo, Z. Wang, and Z. Wang, "pQueue-MAC: an energy efficient hybrid MAC protocol for event-driven sensor networks." IJDSN, vol. 11, pp. 160167:1160167:11, 2015. 
[73] L. G. Roberts, "ALOHA packet system with and without slots and capture," SIGCOMM Comput. Commun. Rev., vol. 5, no. 2, pp. 28-42, Apr. 1975.

[74] R. Rom and M. Sidi, Multiple access protocols: performance and analysis, ser. Telecommunication networks and computer systems. New York: Springer-Verlag, 1990.

[75] D. Bertsekas and R. Gallager, Data Networks (2nd Ed.). Upper Saddle River, NJ, USA: Prentice-Hall, Inc., 1992.

[76] N. Abramson, "THE ALOHA SYSTEM: another alternative for computer communications," in AFIPS '70 (Fall): Proceedings of the November 17-19, 1970, fall joint computer conference. New York, NY, USA: ACM, 1970, pp. 281-285.

[77] L. Kleinrock and F. A. Tobagi, "Packet switching in radio channels: Part i-carrier sense multiple-access modes and their throughput-delay characteristics," IEEE Transactions on Computing, vol. 23, pp. 1400-1416, 1975.

[78] W. Szpankowski, "Packet switching in multiple radio channels: Analysis and stability of a random access system." Computer Networks, vol. 7, pp. 17-26, 1983.

[79] I. E. Pountourakis and E. D. Sykas, "Analysis, stability and optimization of Alohatype protocols for multichannel networks," Computer Communications, vol. 15, no. 10, pp. 619-629, Dec. 1992.

[80] W. Yue, "The effect of capture on performance of multichannel slotted ALOHA systems," IEEE Trans. Communications, vol. 39, pp. 818-822, 1991.

[81] Z. Zhang and Y. Liu, "Comments on 'the effect of capture on performance of multichannel slotted ALOHA systems'," IEEE Trans. Communications, vol. 41, no. 10, pp. 1433-1435, 1993.

[82] O. Galinina, A. Turlikov, S. Andreev, and Y. Koucheryavy, "Stabilizing multi-channel slotted aloha for machine-type communications," in Information Theory Proceedings (ISIT), 2013 IEEE International Symposium on, July 2013, pp. 2119-2123.

[83] R. Motwani and P. Raghavan, Randomized Algorithms. New York, NY, USA: Cambridge University Press, 1995.

[84] L. Comtet, Advanced Combinatorics: The Art of Finite and Infinite Expansions. Springer Netherlands, 1974.

[85] OEIS Foundation Inc. The On-Line Encyclopedia of Integer Sequences, Std., May 2016. [Online]. Available: http://oeis.org/A008277

[86] A. Molisch, Wireless Communications. Wiley-IEEE Press, 2005. 
[87] I. Bilal, A. Meijerink, and M. J. Bentum, in IEEE 25th Annual International Symposium on Personal, Indoor and Mobile Radio Communication, PIMRC 2014, Washington DC, USA. USA: IEEE Antennas \& Propagation Society, September 2014, pp. 727-732.

[88] S. Guha, C.-K. Chau, and P. Basu, "Green wave: Latency and capacity-efficient sleep scheduling for wireless networks." in INFOCOM. IEEE, 2010, pp. 1900-1908.

[89] J. Hill, R. Szewczyk, A. Woo, S. Hollar, D. E. Culler, and K. S. J. Pister, “System architecture directions for networked sensors," in Architectural Support for Programming Languages and Operating Systems, 2000, pp. 93-104.

[90] K. Weniger, "PACMAN: passive autoconfiguration for mobile ad hoc networks," IEEE Journal on Selected Areas in Communications, vol. 23, no. 3, pp. 507-519, 2005.

[91] Z. Yao and F. Dressler, "Dynamic address allocation for management and control in wireless sensor networks." in HICSS. IEEE Computer Society, 2007, p. 292.

[92] F. Ye and R. Pan, "A survey of addressing algorithms for wireless sensor networks," in 2009 5th International Conference on Wireless Communications, Networking and Mobile Computing, Sept 2009, pp. 1-7.

[93] T. S. Rappaport, Wireless Communications: Principles and Practice, 1st ed. Piscataway, NJ, USA: IEEE Press, 1996.

[94] "IEEE standard for information technology-telecommunications and information exchange between systems local and metropolitan area networks-specific requirements part 11: Wireless LAN medium access control MAC and physical layer PHY specifications," IEEE Std 802.11-2012 (Revision of IEEE Std 802.11-2007), pp. 1-2793, March 2012.

[95] P. Karn, "MACA-a new channel access method for packet radio," in $A R R L / C R R L$ Amateur radio 9th computer networking conference, vol. 140, 1990, pp. 134-140.

[96] A. El-Hoiydi, "Energy efficient medium access control for wireless sensor networks," PhD dissertation, EPF Lusanne, 2005. 



\section{About the author}

Sarwar Morshed was born in Dhaka, Bangladesh in 1984. He has obtained HIS B.Sc in Computer Science in Information Technology from Islamic University of Technology, Bangladesh in 2005. Afterwards, he joined as his alma mater as a Lecturer. Later on, he obtained his M.Sc. in Information Technology from Tampere University of Technology, Finland in 2009. After obtaining his M.Sc., he again continued his work in Islamic University of Technology, Bangladesh.

Sarwar joined the Design and Analysis of Communications Systems research group of University of Twente in 2012. During his PhD, he worked in the WALNUT (Wireless Ad-hoc Links using robust Noise-based Ultra-wideband Transmissions) project funded by the Dutch Technology Foundation (STW). The WALNUT project had industry partners NXP semiconductors, Thales, TNO, Plantronics, DevLab, and STmicroelectronics. Three research groups within University of Twente worked in this project to realize the TR modulation in circuit implementation, analyzing the modulation characteristics in the physical layer, and finally modelling a MAC layer communication protocol on top. Sarwar created the scope of a new energy-efficient medium access control (MAC) layer communication protocol for wireless sensor networks exploiting the transmit reference (TR) modulation in underlying physical layer. This protocol is called transmit reference-medium access control (TR-MAC), which provides energy-efficient connectivity with traffic-adaptive behavior together with multiple access control. Presently, Sarwar works at ASML Netherlands B.V. in Veldhoven, The Netherlands.

List of Publications in reverse-chronological order:

- Morshed, S. and Bilal, I. and Ye, D. and Heijenk, G.J. and Meijerink, A. and Zee, R. V. D. and Bentum, M. (2017) An overview of frequency offset based transmit reference system for wireless sensor networks. Manuscript in process for submission in IEEE Communications Magazine 2017.

- Morshed, S. and Baratchi, M. and Mandal. P. K. and Heijenk, G.J. (2016) A multichannel Multiple Access Scheme for Wireless Sensor Networks Using Frequency 
Offsets - Modelling and Analysis. In: Proceedings of the 12th International Conference on Wireless and Mobile Computing, WiMob 2016, Networking and Communications 2016, 17-19 October, New York, USA.

- Morshed, S. and Baratchi, M. and Heijenk, G.J. (2016) Traffic-adaptive duty cycle adaptation in TR-MAC protocol for Wireless Sensor Networks. In: Proceedings of the 8th International Conference on Wireless Days 2016, 23-25 March, Toulouse, France.

- Morshed, S. and Heijenk, G.J. (2015) Optimization and Verification of the TR-MAC Protocol for Wireless Sensor Networks. In: Proceedings of the 13th International Conference on Wired/Wireless Internet Communications, WWIC 2015, Revised Selected Papers, May 25-27, 2015, Malaga, Spain. pp. 396-410.

- Morshed, S. and Heijenk, G.J. (2015) TR-MAC: An energy-efficient MAC protocol for wireless sensor network. In: ICT.OPEN 2015, 24 Mar 2015, Amersfoort, The Netherlands.

- Morshed, S. and Heijenk, G.J. (2014) TR-MAC: an energy-efficient MAC protocol exploiting transmitted reference modulation for wireless sensor networks. In: Proceedings of the 17th ACM International Conference on Modeling, Analysis and Simulation of Wireless and Mobile Systems, MSWiM 2014, 21-25 Sep 2014, Montreal, Canada. pp. 21-29.

- Morshed, S. and Heijenk, G.J. (2013) TR-MAC: an energy-efficient MAC protocol for wireless sensor networks exploiting noise-based transmitted reference modulation. In: 2nd Joint ERCIM eMobility and MobiSense Workshop, 4 Jun 2013, St. Petersburg, Russia. pp. 58-71.

- Morshed, S. and Heijenk, G.J. and Meijerink, A. and Ye, Dawei and van der Zee, R.A.R. and Bentum, M.J. (2013) A new energy-efficient MAC protocol with noisebased transmitted-reference modulation for wireless sensor network. In: Sense of Contact 15 - Sensors across the application domains, 10 Apr 2013, Soesterberg, The Netherlands. pp. 1-2. Dutch Technology Foundation (STW).

- Morshed, S. and Heijenk, G.J. and Meijerink and Mahboob M. and Bentum, M.J. (2012) Noise Based Transmitted Reference Modulation for Wireless Sensor Networks. In: Sense of Contact 14 - Sensors across the application domains, 2 Apr 2012, Soesterberg, The Netherlands.pp. 1-2. Dutch Technology Foundation (STW). (Best paper award) 


\section{Acronyms}

$\begin{array}{ll}\text { BPSK } & \text { Binary Phase Shift Keying } \\ \text { CDMA } & \text { Code Division Multiple Access } \\ \text { CSMA } & \text { Carrier Sense Multiple Access } \\ \text { CSMA/CA } & \text { Carrier Sense Multiple Access / Collision Avoidance } \\ \text { CTS } & \text { Clear To Sender } \\ \text { FDMA } & \text { Frequency Division Multiple Access } \\ \text { IoT } & \text { Internet of Things } \\ \text { LPL } & \text { Low Power Listening } \\ \text { MAC } & \text { Medium Access Control } \\ \text { M2M } & \text { Machine to Machine } \\ \text { OSI } & \text { Open Systems Interconnections } \\ \text { QoS } & \text { Quality of Service } \\ \text { RTS } & \text { Request To Send } \\ \text { SDMA } & \text { Space Division Multiple Access } \\ \text { SNIR } & \text { Signal to Noise and Interfernce Ratio } \\ \text { TDMA } & \text { Time Division Multiple Access } \\ \text { TR } & \text { Transmit Reference } \\ \text { UWB } & \text { Ultra-Wide Band } \\ \text { WSN } & \text { Wireless Sensor Network }\end{array}$






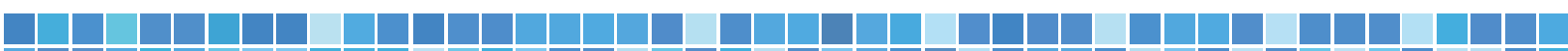

\begin{tabular}{lll}
\hline &
\end{tabular} (1 10 $\square$

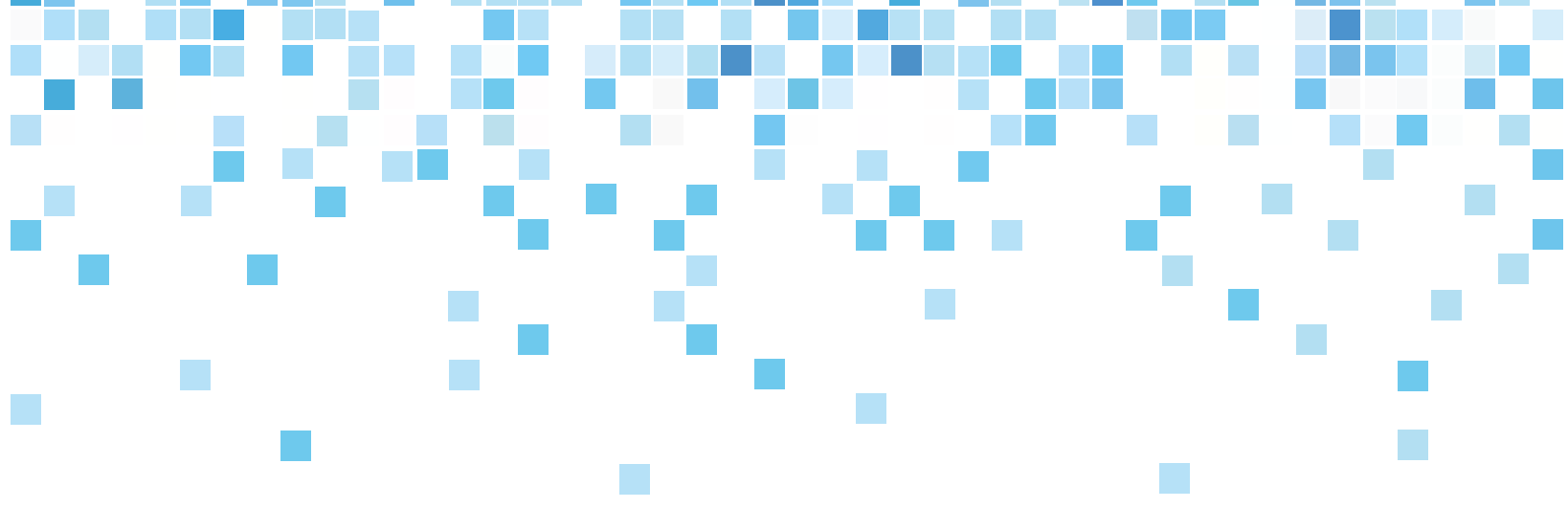

$\square$

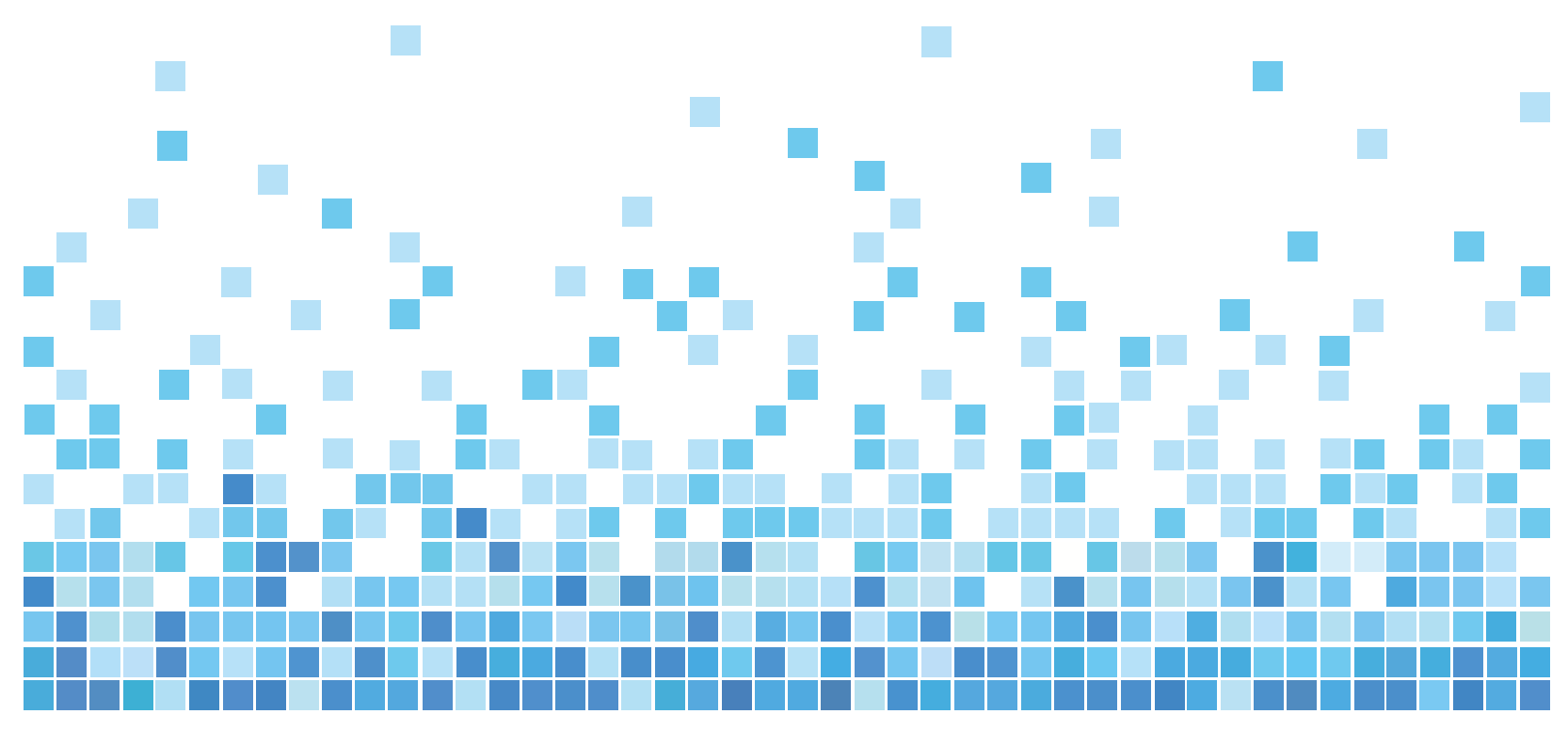

